\title{
Tight List-Sizes for Oblivious AVCs under Constraints
}

\author{
Yihan Zhang*, Sidharth Jaggi ${ }^{\dagger *}$, Amitalok J. Budkuley ${ }^{\ddagger}$ \\ *Dept. of Information Engineering, The Chinese University of Hong Kong \\ ${ }^{\dagger}$ School of Mathematics, University of Bristol \\ $\ddagger$ Dept. of Electronics and Electrical Communication Engineering, Indian Institute of Technology \\ Kharagpur
}

\begin{abstract}
We study list-decoding over adversarial channels governed by oblivious adversaries (a.k.a. oblivious Arbitrarily Varying Channels (AVCs)). This type of adversaries aims to maliciously corrupt the communication without knowing the actual transmission from the sender. For any oblivious AVCs potentially with constraints on the sender's transmitted sequence and the adversary's noise sequence, we determine the exact value of the minimum list-size that can support a reliable communication at positive rate. This generalizes a classical result by Hughes (IEEE Transactions on Information Theory, 1997) and answers an open question posed by Sarwate and Gastpar (IEEE Transactions on Information Theory, 2012). A lower bound on the list-decoding capacity (whenever positive) is presented. Under a certain combinatorial conjecture, we also prove a matching upper bound. En route to a tight characterization of the list-decoding capacity, we propose a method for subcode construction towards the resolution of the combinatorial conjecture.
\end{abstract}

\section{INTRODUCTION}

Coding against adversaries is one of the central subjects in coding theory and information theory. The canonical model of interest is as follows. Suppose a transmitter Alice would like to send a message to a receiver Bob through a noisy channel. The channel is governed by an adversary (also called a jammer) James whose aim is to maliciously corrupt the data transmission from Alice to Bob. Coding is a technique to protect data from corruption and thus ensures a reliable communication. Alice, instead of sending the message per se, introduces some redundancy to the message and sends an encoded sequence (called codeword). James carefully designs a jamming sequence (historically a.k.a. state sequence) and transmits it to the channel. The hope is that even James adversarially distorted Alice's transmitted codeword via the channel, Bob receiving the noisy channel output is still able to reliably decode. The goal is to find the maximum information throughput (a.k.a. rate) of a given channel, i.e., the largest number of bits that could be delivered with a low probability of reconstruction error. This paper is concerned with characterizing information-theoretic fundamental limits (called capacity) of this kind assuming there is no restriction on the computational power of Alice/Bob/James. See Section VI for formal definitions.

The aforementioned adversarial communication channel model is often referred to as the Arbitrarily Varying Channel (AVC) model, first introduced by Blackwell, Breiman and Thomasian [BBT60]. It contains a large family of channels of interests and has received a lot of study since it was proposed. It turns out that in an adversarial communication problem, it is important to clarify the power of James. The knowledge that James possesses when designing the jamming vector is an important component of the problem setup. There are two natural and popular models that are well-studied in the literature. The omniscient model assumes that James knows precisely which particular codeword was transmitted by Alice. Hence he could look at the transmission and tailor his jamming strategy for a specific instantiation of transmission. This is a very strong type of adversaries and the capacity of such AVCs is widely open. Another model called the oblivious model instead assumes that the adversary does not know at all which codeword was transmitted by Alice. Said differently, one can think of James as choosing a jamming sequence before Alice's transmission is instantiated. Much more capacity results are known in this setting. The oblivious model is the main focus of this paper. See [LN98] for a survey on AVCs and see Section II for prior works.

We now shift our attention from James to Bob. The reliability of communication can be measured in various different ways. The metric that was alluded to in the first paragraph of this section is called unique-decodability. As 
the name suggests, Bob is required to, based on his received vector, decode to a single message which is hopefully the correct one that Alice meant to deliver. In some scenarios, this is too stringent a requirement to satisfy or it is much trickier to directly prove without intermediate steps. One possible notion of reliability that relaxes this condition is called list-decodability. This allows Bob to output a list of messages which should contain the correct one. Of course, to avoid triviality, we would like the list to be as small as possible. The notion of list-decoding was first proposed by Elias [Eli57] and Wozencraft [Woz58]. It has since received a lot of study from various aspects and has become an important subject within and outside the scope of Coding \& Information Theory. In Computer Science, list-decoding is most well-studied under the omniscient bitflip channel model. See [Gur04], [Gur07] for a comprehensive survey on combinatorial and algorithmic results on list-decoding of this flavour. Listdecoding also serves as a primitive in complexity theory and cryptography, e.g., [DMOZ19]. Recently, the idea of relaxing the problem by allowing the solver to return a list of answers rather than a unique answer also goes into the development of robust statistics [DKS18], [DKK20], [CMY20], [KKK19], [BK20], [RY20b], [RY20a]. In our context, given an adversarial channel, one of the fundamental questions is to pin down the smallest list-size $L^{*}$ (a.k.a. list-symmetrizability) that supports a reliable communication at positive rate. Understanding capacity positivity is the first step towards understanding the exact value of the capacity of an AVC under list-decoding. In this paper, for any oblivious AVC, we give a tight characterization on the exact value of the minimum list-size such that the list-decoding capacity w.r.t. such a list-size is strictly positive. See Section III for an overview of our results.

Finally, as a technical note, we emphasize that in this paper we use the average probability of error ${ }^{1}$ criterion, i.e., a $1-o(1)$ (but not exactly 1) fraction of messages are required to be correctly decoded. ${ }^{2}$ Also, we do not assume common randomness available to Alice and Bob (but secret to James). AVC problems under other probability of error criteria and/or in the presence of common randomness are also interesting. See Section II for related works.

\section{PRIOR WORK}

\section{A. Oblivious AVCs}

For AVCs, one of the challenges of characterizing the capacity is perhaps to first determine whether the capacity is zero or strictly positive. For general AVCs, finding a sufficient and necessary condition for achieving positive rate turns out to be highly nontrivial. Such conditions are usually called symmetrizability in the literature. We explain the underlying intuition using the oblivious bitflip channels which are perhaps the simplest nontrivial example of oblivious adversarial channels. As shown in Figure 1, both the transmitted codeword $\underline{\mathbf{x}}$ and the jamming sequence

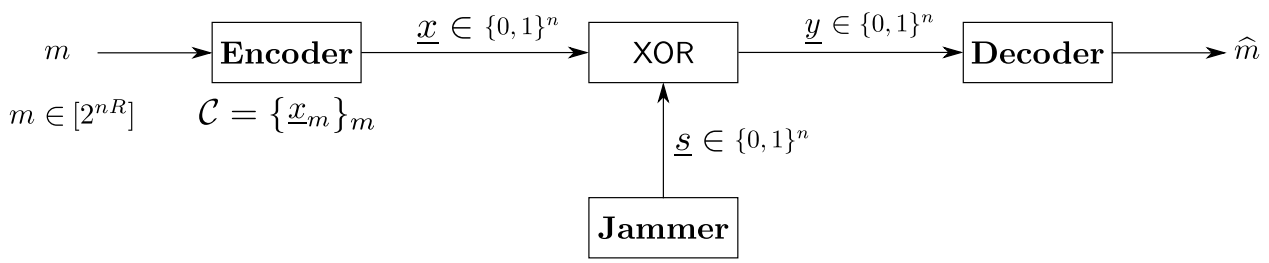

Fig. 1: Block diagram of an oblivious bitflip channel.

$\underline{\mathbf{s}}$ are $\{0,1\}^{n}$-valued. The channel in this case is simply a deterministic function that adds up $\underline{\mathbf{x}}$ and $\underline{\mathbf{s}}$ componentwise modulo 2. The channel output is therefore $\underline{\mathbf{y}}=\underline{\mathbf{x}} \oplus \underline{\mathbf{s}}$. Though James does not know the value of $\underline{\mathbf{x}}$, he could transmit a uniformly random codeword $\underline{x}^{\prime} \sim \mathcal{C}$ from the codebook (which is assumed to be known to everyone). If $\underline{\mathbf{x}}^{\prime} \neq \underline{\mathbf{x}}$ (which does happen with high probability as long as the code is large), Bob receives $\underline{\mathbf{y}}=\underline{\mathbf{x}} \oplus \underline{\mathbf{x}}^{\prime}$ and could not distinguish whether Alice transmitted $\underline{\mathrm{x}}$ or $\underline{\mathrm{x}}^{\prime}$. In other words, the scenario where Alice transmits $\underline{\mathrm{x}}$ and James transmits $\underline{\mathrm{x}}^{\prime}$ and the scenario where Alice transmits $\underline{\mathrm{x}}^{\prime}$ and James transmits $\underline{\mathrm{x}}$ result in the same statistics at

\footnotetext{
${ }^{1}$ The average refers to averaging over messages that Alice can potentially transmit. They are assumed to be uniformly distributed.

${ }^{2}$ There exist other notions of error criteria which are also interesting and have been studied in the literature. One other important one is the maximum probability of error criterion. Again, the maximization is over messages. Under deterministic encoders, the problem is equivalent to the omniscient case since we can assume that the adversary knows the transmitted message and hence the transmitted codeword. Under stochastic encoders (which is possible if Alice has access to private randomness), it can be shown that the capacity remains the same as that under the average probability of error criterion.
} 


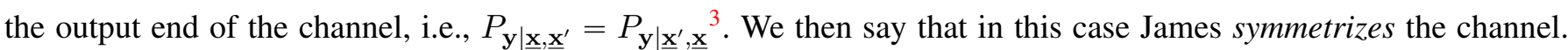
This forces Bob to fail to decode the correct message with constant probability and hence forces the capacity to be zero. Symmetrizability is a fundamental characteristic associated to an AVC. Given a generic AVC, it is not obvious how to capture this kind of phenomena using a precise notion of symmetrizability. The seminal work by Csiszár and Narayan [CN88b] provided the right notion of symmetrizability of a general oblivious AVC and used it to give a characterization of the capacity. [CN88b]'s notion of symmetrizability is a certain linear algebraic condition that can be easily verified for any given oblivious AVC. Remarkably, such a condition was not available previously even for AVCs without input or state constraints [Ahl78], i.e., Alice and James are allowed to transmit any length- $n$ sequence over their alphabets.

The quadratically constrained version of the oblivious AVC problem is also well-studied. In this variant, vectors in the communication system are $\mathbb{R}^{n}$-valued subject to $\ell^{2}$-norm constraints. The capacity of such channels (a.k.a. Gaussian AVCs) was obtained in [CN91] via a geometric approach.

For list-decoding in the oblivious setting, without input and state constraints, the notion of list-symmetrizability (formally defined in Definition 5) was given in a paper by Hughes [Hug97] and the L-list-decoding capacity (formally defined in Definition 4) for any list-size $L \in \mathbb{Z}_{\geqslant 1}$ was characterized therein. As we shall see in more details in Section III, incorporating constraints (especially state constraints) into the definition of symmetrizability (and list-symmetrizability) is nontrivial. Intuitively, this is because symmetrizability is a symbol-wise notion. For simplicity, we explain the effect of constraints using unique-decoding as an example. If a pair of input symbols $\left(x, x^{\prime}\right) \in \mathcal{X}^{2}$ are "confusable" in the symmetrizability sense that $P_{\mathbf{y} \mid x, x^{\prime}}=P_{\mathbf{y} \mid x^{\prime}, x}$, then the encoder should avoid using them, since $x$ and $x^{\prime}$ will cause confusion to Bob and receiving symbol $\mathbf{y}$ in certain locations, he not could tell if the value of the original transmission was $x$ or $x^{\prime}$ in these locations. If all pairs of input symbols are confusable, then Alice has no effective symbols that can be used for communication without causing confusion at the decoder end. Hence the symbol-wise notion of symmetrizability translates to an operational notion of confusability at the level of vectors $\left(P_{\underline{\mathbf{y}} \mid \underline{\mathbf{x}}, \underline{\mathbf{x}}^{\prime}}=P_{\underline{\mathbf{y}} \mid \underline{\mathbf{x}}^{\prime}, \underline{\mathbf{x}}}\right)$. However, in the presence of state constraints, even if all pairs of symbols are confusable, it does not immediately mean that James can always confuse Bob w.r.t two different codewords. In fact, this is known to be not true [CN88b]. In the oblivious bitflip example, if we further impose constraints on $\underline{x}$ and $\underline{s}$ such that $w t_{\mathrm{H}}(\underline{x}) \leqslant w$ and $w t_{\mathrm{H}}(\underline{s}) \leqslant p$ for some $w, p \in[0,1 / 2]$, where $w t_{\mathrm{H}}(\cdot)$ denotes the Hamming weight of a vector, then the capacity is positive if (and only if) $w>p$. However, the only two input symbols 0 and 1 are apparently confusable. This phenomenon is precisely due to the effect of state constraints. If James is only allowed to jam using some rather than arbitrary sequences, then symbol-wise symmetrizability does not rule out the possibility that Alice could still communicate to Bob at a positive rate while being robust to all James's feasible jamming sequences. Indeed, it was first observed by Ericson [Eri85] that unconstrained AVCs are impractical models since many channels of interests are symmetric (e.g., the bitflip channels discussed above) and hence by definition is symmetrizable and has zero capacity. Constrained oblivious AVCs under list-decoding were studied by Sarwate and Gastpar in [SG12]. They gave upper and lower bounds on $L^{*}-$ the smallest list-size $L$ such that the $L$-listdecoding capacity is positive. In particular, they defined the notions of weak and strong list-symmetrizability such that $L_{\text {strong }}^{*} \leqslant L^{*} \leqslant L_{\text {weak }}^{*}$. Using these notions, they prove natural lower and upper bounds on the $L$-list-decoding capacity.

The quadratically constrained version of the oblivious AVC problem under list-decoding turns out to be easier due to the Euclidean nature. Hosseinigoki and Kosut recently [HK18] characterized the $L$-list-decoding capacity of such channels. The achievability uses typicality methods for real-valued vectors. A natural list-symmetrization strategy was analyzed using a certain novel bounding technique.

\section{B. Omniscient AVCs}

Much less is known in the omniscient setting. Let us first look at capacity positivity. For omniscient AVCs without input/state constraints, it is well-known that the capacity is zero if and only if the channel is symmetrizable in the sense of Kiefer and Wolfowitz [KW62]. Again, incorporating constraints is nontrivial in the omniscient case as well. Until very recently, Wang, Budkuley, Bogdanov and Jaggi [WBBJ19] managed to get the right notion of

\footnotetext{
${ }^{3}$ We use boldface lowercase letters to denote (scalar) random variables, plain lowercase letters to denote their realizations and underlines to denote vectors of length $n$, where $n$ is the blocklength of the code unless otherwise specified. See Section V for the notational convention followed in this paper.
} 
symmetrizability (a.k.a. confusability in their paper) for general omniscient AVCs with input \& state constraints whose channel transition distribution is a 0-1 law, i.e., the channel output is a deterministic function of Alice's and James's inputs. They showed that such channels have zero capacity if and only if they are symmetrizable in [WBBJ19]'s sense. Their results are a significant generalization of the Plotkin bound in classical coding theory. Remarkably, the connection between the complete positivity of joint distributions and the structure of codes was first introduced in [WBBJ19]. With additional work, their techniques can potentially carry over to the case where $W_{\mathbf{y} \mid \mathbf{x}, \mathbf{s}}$ is an arbitrary distribution. However, a formal proof has not been presented yet.

We now turn to bounds on the capacity. As we shall see below, the situation in this direction is rather sad and essentially no capacity result is known even for very simple channels. For general omniscient AVCs without input/state constraints, Csiszár and Körner [CK81] proved a lower bound on the capacity using techniques similar to those used in [CN88b] for oblivious channels. For channels with input \& state constraints and with $0-1$ transition distributions, a Gilbert-Varshamov-type lower bound and its natural generalization using time-sharing were presented in [WBBJ19]; an Elias-Bassalygo-type upper bound will be presented in the full version of [WBBJ19] which is not yet available when the present paper is written. In classical coding theory, the most commonly considered model is the omniscient bitflip channel. The capacity of such a channel is equivalent to the largest sphere packing density in Hamming space. The best lower and upper bounds so far are the Gilbert-Varshamov bound [Gil52], [Var57] and the second MRRW (Linear Programming-type) bound [MRRW77], respectively. They match nowhere except when the channel is completely noiseless or noisy, which are trivial cases. The exact value of the capacity is perhaps the most central open question in coding theory.

The quadratically constrained version of the channel coding problem for omniscient AVCs is equivalent to packing caps on a sphere. In the latter problem, one would like to pack as many as possible mutually disjoint spherical caps of radii $\sqrt{n N}$ on a sphere of radius $\sqrt{n P}$. It is well-known that one can achieve positive packing density whenever $N / P<1 / 2$. The current best lower and upper bounds on the packing density (or the capacity of a quadratically constrained omniscient AVC) are the Gilbert-Varshamov-type lower bound due to Blachman [Bla62] and the Linear Programming-type upper bound due to Kabatiansky and Levenshtein [KL78], respectively. Narrowing the gap from either side is regarded as a big breakthrough.

For list-decoding against omniscient adversaries, the situation is similar. For general channels with input \& state constraints and with 0-1 transition distributions, Zhang, Budkuley and Jaggi [ZBJ20] recently extended the results of [WBBJ19] to list-decoding and provided a sufficient and necessary condition for positivity of the $L$-list-decoding capacity for any given list-size $L \in \mathbb{Z}_{\geqslant 1}$. In the same paper, the authors also gave lower bounds on the $L$-listdecoding capacity using random coding with expurgation. An Elias-Bassalygo-/Johnson-type upper bound will also be presented in the full version of [ZBJ20] (which is not yet available when the present paper is written). These lower and upper bounds for general omniscient AVCs are generalizations of similar bounds for the bitflip channels due to a sequence of beautiful works by Blinovsky [Bli86], [Bli05], [Bli08]. Blinovsky's upper bounds were improved by Polyanskiy [Pol16] in the high-rate regime for odd list-sizes at least 3.

The quadratically constrained version of list-decoding is equivalent to packing caps on a sphere with bounded multiplicity of overlap (a.k.a. multiple packing). To the best of the authors' knowledge, the current best lower and upper bounds are due to Blinovsky [Bli97] and Blinovsky-Litsyn [BL09], respectively. The largest multiple packing density also remains elusive.

\section{Zero-error information theory}

The omniscient model is intimately related to "noiseless" channels under zero-error criterion. It can be shown that for omniscient channels, without loss of rate, all error criteria are equivalent to the zero-error criterion. That is, the capacity under average/maximum error criterion is the same as that with the requirement that the decoder makes an error with probability precisely (rather than asymptotically) zero. The problem of characterizing the capacity is hence of combinatorial nature. If the channel is "noiseless" in the sense that the adversary is absent, then the problem falls into the realm of zero-error information theory [Sha56]. Though without noise, the zero-error capacity of a channel $W_{\mathbf{y} \mid \mathbf{x}}$ is still widely open. It is well known to be equal to $\lim _{n \rightarrow \infty} \alpha\left(\mathcal{G}\left(W_{\mathbf{y} \mid \mathbf{x}}\right)^{\llbracket n}\right)^{1 / n}$, where $\mathcal{G}\left(W_{\mathbf{y} \mid \mathbf{x}}\right)$ is the confusability graph of $W_{\mathbf{y} \mid \mathbf{x}}, \mathcal{G}^{\bowtie n}$ denotes the $n$-fold strong product of $\mathcal{G}$ and $\alpha(\mathcal{G})$ denotes the independence number of $\mathcal{G}$. This characterization is not single-letter and hence is not computable (since it involves a limit as the blocklength $n$ of the code grows). This formula is only successfully evaluated for sporadic nontrivial channels, 
e.g., the noisy typewriter channel with alphabet size five [Lov79]. Determination of the zero-error capacity of even the noisy typewriter channel with alphabet size seven remains formidable, let alone general channels. Nevertheless, we emphasize that the capacity positivity of this problem $i$ s understood. Indeed, it is well-known and not hard to see that the capacity is positive if and only if the confusability graph of the channel is not a complete graph. See [KO98] for a survey on zero-error information theory.

\section{Other exotic models}

Other types of adversaries such as myopic adversaries [DJL19b], [ZVJS18], [BDJ $\left.{ }^{+} 20\right],\left[\mathrm{DJL}^{+} 19 \mathrm{a}\right]$, causal/online adversaries [DJLS13], [CJL15], [LDJ ${ }^{+}$18], adversaries with delay, adversaries with lookahead [DJLS16]; and other types of channels such as channels with feedback [Ber64], [Zig76], [AC00], [HKV15], channels with common randomness [CN88a], [Ahl78], two-way adversarial channels [JL17], [ZVJ20], adversarial broadcast channels [PS17], [HK20], adversarial interference channels [HK16], adversarial relay channels [PS19a], [PS18], adversarial Multiple Access Channels (MACs) [Jah81], [AC99], [PS19b], adversarial fading channels [HK19] etc. were also studied in the literature. In each of these models, the adversaries may exhibit starkly contrasting behaviours. We do not intend to provide an exhaustive list of prior works.

\section{OVERVIEW OF OUR RESULTS AND TECHNIQUES}

In this work, we provide the correct notion of list-symmetrizability $L^{*}$ which we call CP-symmetrizability, denoted by $L_{\mathrm{CP}}^{*}$. We show $L^{*}=L_{\mathrm{CP}}^{*}$ and prove bounds on the $L$-list-decoding capacity for any $L \in \mathbb{Z}_{\geqslant 1}$ using this new notion of list-symmetrizability. (See Theorem 11 for formal statements.) Specifically, given any oblivious AVC with input \& state constraints, we show the following.

1) When a given target list-size $L$ is at most $L_{\mathrm{CP}}^{*}$, then the channel is $L$-symmetrizable and the $L$-list-decoding capacity is zero. See Theorem 12 for a formal statement.

2) When $L$ is strictly greater than $L_{\mathrm{CP}}^{*}$, we prove a natural lower bound on the $L$-list-decoding capacity using techniques that slightly extends those in [CN88b], [Hug97], [SG12]. In particular, the capacity is positive in this case. See Theorem 13 for a formal statement.

3) When $L$ is strictly greater than $L_{C P}^{*}$, we did not manage to prove a matching upper bound on capacity. En route to a tight characterization, we propose a conjecture conditioned on which we show that our lower bound is tight. See Theorem 14 for a formal statement. The conjecture (Conjecture 21) is concerned with basic structures of sets of vectors (over finite alphabets). It is of combinatorial nature and does not require backgrounds in AVCs. We propose a natural subcode construction (Section XI-C) towards the resolution of this conjecture.

Curiously, our proof techniques crucially hinge on the recent development in the study of omniscient AVCs. As alluded to in Section II-A, incorporating constraints (especially state constraints) into Hughes's [Hug97] definition of list-symmetrizability is a challenging task. Sarwate and Gastpar [SG12] made the first attempt by providing two candidates (strong and weak list-symmetrizability, denoted by $L_{\text {strong }}^{*}$ and $L_{\text {weak }}^{*}$, respectively) of extension of Hughes's notion to the constrained case. However, these extended notions are not tight in the sense of dichotomy, i.e., for any given list-size $L \in \mathbb{Z}_{\geqslant 1}$, the $L$-list-decoding capacity is zero if $L \leqslant L^{*}$ and is positive otherwise.

We now explain how we close the gap between $L_{\text {strong }}^{*}$ and $L_{\text {weak }}^{*}$ using the notion of CP-symmetrizability $L_{\mathrm{CP}}^{*}$ (where CP stands for completely positive). We first equip James with an improved jamming strategy (called (P-symmetrization) which allows him to enforce a zero communication rate. Fix a list-size $L \in \mathbb{Z}_{\geqslant 2}{ }^{4}$ and a code $\mathcal{C} \subseteq \mathcal{X}^{n}$ of positive rate ${ }^{5}$ satisfying input constraints. Suppose Alice transmitted a codeword $\underline{\mathbf{x}}_{i_{0}} \sim \mathcal{C}$ corresponding to a random message $i_{0}$. As suggested by the intuition mentioned in Section II-A, a natural way to "symmetrize" the channel is to let James sample a "spoofing" list $\mathcal{L}:=\left(\underline{\mathbf{x}}_{i_{1}}, \cdots, \underline{\mathbf{x}}_{i_{L}}\right)$ of $L$ codewords uniformly from $\mathcal{C}$ such that the output distribution looks identical if the $(L+1)$-list $\mathcal{L}^{\prime}:=\left(\underline{\mathbf{x}}_{i_{0}}, \underline{\mathbf{x}}_{i_{1}}, \cdots, \underline{\mathbf{x}}_{i_{L}}\right)$ is permuted arbitrarily, i.e.,

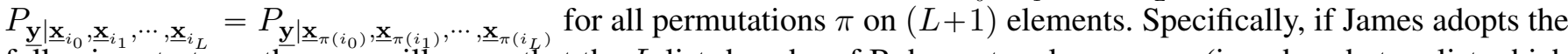
following strategy, then we will argue that the $L$-list-decoder of Bob must make an error (i.e., decode to a list which does not contain the truly transmitted message) with nonvanishing probability. If we provide James with a (discrete memoryless) "jamming channel" $U_{\mathbf{s} \mid \mathbf{x}_{1}, \cdots, \mathbf{x}_{L}}$, then he can use it to generate a random jamming sequence $\underline{\mathbf{s}}_{\mathcal{L}}$ once the

\footnotetext{
${ }^{4}$ When $L=1$, the problem at hand collapses to the unique-decoding problem which was solved in [CN88b].

${ }^{5}$ Positive rate of a code $\mathcal{C} \subseteq \mathcal{X}^{n}$ simply means the code size is exponentially large in $n$, i.e., $|\mathcal{C}|=|\mathcal{X}|^{n R}$ for some constant $R \in(0,1]$.
} 
spoofing list $\mathcal{L}$ is fed into it. Suppose we can find a $U_{\mathbf{s} \mid \mathbf{x}_{1}, \cdots, \mathbf{x}_{L}}$ satisfying the following property. The distribution of the channel output $\underline{\mathbf{y}}$ obtained from $\left(\underline{\mathbf{x}}_{i_{0}}, \underline{\mathbf{s}}_{\mathcal{L}}\right) \stackrel{W_{\mathbf{y} \mid \mathbf{x}, \mathbf{s}}}{\longrightarrow} \underline{\mathbf{y}}\left(\right.$ where $\left.\mathcal{L} \stackrel{U_{\mathbf{x}_{1}, \cdots, \mathbf{x}_{L}}^{\otimes n}}{\longrightarrow} \underline{\mathbf{s}}_{\mathcal{L}}\right)$ remains the same if $\underline{\mathbf{y}}$ is obtained from $\left(\underline{\mathbf{x}}_{\pi\left(i_{0}\right)}, \underline{\mathbf{s}}_{\pi(\mathcal{L})}\right) \stackrel{W_{\mathbf{y} \mid \mathbf{x}, \mathbf{s}}^{\otimes}}{\longrightarrow} \mathbf{y}\left(\right.$ where $\pi(\mathcal{L}) \stackrel{U_{\mathbf{s} \mid \mathbf{x}_{1}, \cdots, \mathbf{x}_{L}}^{\otimes n}}{\longrightarrow} \underline{\mathbf{s}}_{\pi(\mathcal{L})}$ and $\left.\pi(\mathcal{L}):=\left(\underline{\mathbf{x}}_{\pi\left(i_{1}\right)}, \cdots, \underline{\mathbf{x}}_{\pi\left(i_{L}\right)}\right)\right)$ for any permutation $\pi$ on $(L+1)$ elements. For example, if $L=2$, this property guarantees that $\underline{\mathbf{y}}_{1}, \cdots, \underline{\mathbf{y}}_{6}$ (where $6=(2+1)$ !) are statistically identical, where $\underline{\mathbf{y}}_{1}, \cdots, \underline{\mathbf{y}}_{6}$ follow the Bayesian networks shown in Figure 2 , respectively. As a
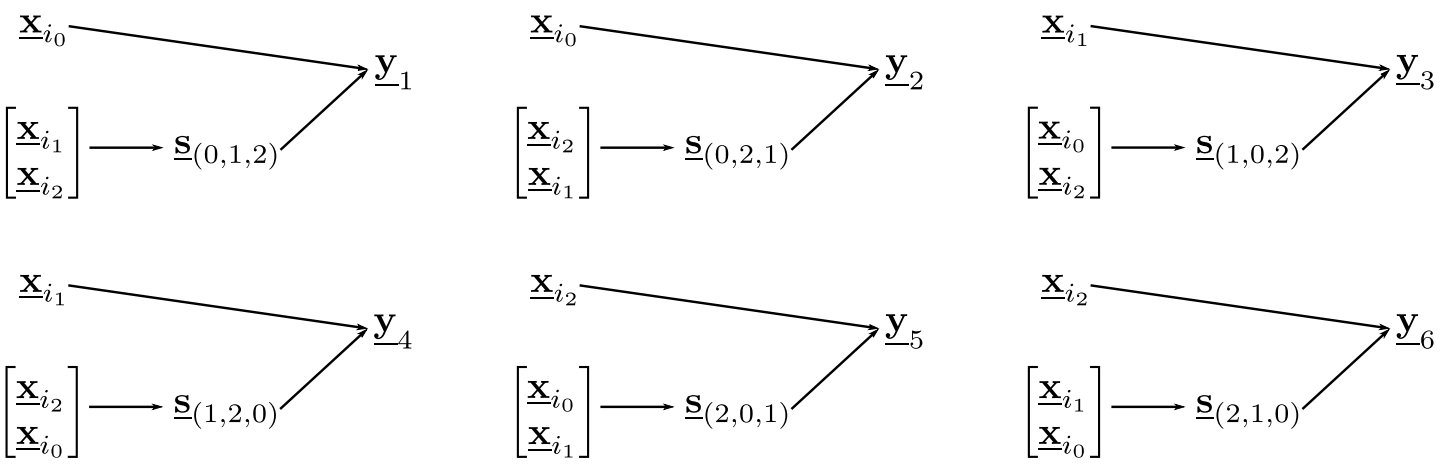

Fig. 2: Example of list-symmetrizability.

consequence of such a property of $U_{\mathbf{s} \mid \mathbf{x}_{1}, \cdots, \mathbf{x}_{L}}$, every $L$-sized sublist of $\mathcal{L}^{\prime}$ appears to be equally likely a posteriori. Bob's best list-decoder is essentially to output a random $L$-sublist $\widehat{\mathcal{L}}$ in $\mathcal{L}^{\prime}$. The probability that Alice's transmitted message $i_{0}$ falls outside $\widehat{\mathcal{L}}$ is about $\frac{1}{L+1}$. For constant list-size $L$, the probability of error is bounded away from zero. In the above example, among messages in the the candidate list $\left\{i_{0}, i_{1}, i_{2}\right\}$, Bob randomly outputs two messages. The probability that he chooses $\left\{i_{1}, i_{2}\right\}$ (which does not contain message $i_{0}$ ) instead of $\left\{i_{0}, i_{1}\right\}$ or $\left\{i_{0}, i_{2}\right\}$ (which are desirable output lists) is $1 / 3>0$. This means that James has successfully jammed the communication and no positive rate of $L$-list-decodable codes with vanishing average probability of error can be achieved ${ }^{6}$ under this type of jamming strategy. It turns out the above heuristic strategy can be formalized to give a sufficient condition for zero list-decoding capacity in the absence of constraints [Hug97].

For general oblivious AVCs under constraints, one caveat in the above heuristics is that we also need to ensure that $\underline{\mathbf{s}}_{\pi(\mathcal{L})}$ satisfies the state constraints for every permutation $\pi$ on $(L+1)$ elements. Note that the distribution of $\underline{\mathbf{s}}_{\pi(\mathcal{L})}$ is essentially (with high probability tightly concentrated around) $P_{\underline{\mathbf{x}}_{\pi\left(i_{0}\right)}, \underline{\mathbf{x}}_{\pi\left(i_{1}\right)}, \cdots, \underline{\mathbf{x}}_{\pi\left(i_{L}\right)}} U_{\mathbf{s} \mid \mathbf{x}_{1}, \cdots, \mathbf{x}_{L}}^{\otimes n}$ marginalized to $\underline{\mathbf{s}}$. A priori, it is unclear whether this distribution remains (approximately) the same under different permutations. Thus it is challenging to bound the distribution of the jamming sequence. Therefore, in the presence of state constraints, we cannot yet claim that the above jamming strategy works. We bypass this obstacle by invoking a list-decoding version of generalized Plotkin bound recently developed by Zhang, Budkuley and Jaggi [ZBJ20] (which in turn built upon [WBBJ19]). This theorem (informally stated below) is concerned with basic structures of sets of vectors over finite alphabets (in particular, codes).

Theorem 1 ((A corollary of) generalized Plotkin bound for list-decoding, informal, [ZBJ20]). Let $\mathcal{X}$ be a finite alphabet. In any sufficiently large $e^{7}$ set $\mathcal{C}=\left\{\underline{x}_{i}\right\}_{i}$ of vectors in $\mathcal{X}^{n}$, there is a completely positive distribution $P_{\mathbf{x}_{1}, \cdots, \mathbf{x}_{L}}$ such that, with constant (independent of $n$ ) probability, a uniformly random (ordered) list $\left(\underline{\mathbf{x}}_{i_{1}}, \cdots, \underline{\mathbf{x}}_{i_{L}}\right)$ $\left(i_{1}<\cdots<i_{L}\right)$ has empirical distribution ${ }^{8}$ approximately equaling $P_{\mathbf{x}_{1}, \cdots, \mathbf{x}_{L}}$.

Remark 1. What was actually proved in [ZBJ20] is a Plotkin-type bound giving rise to a sufficient and necessary condition for list-decoding capacity positivity of general omniscient AVCs. It can be viewed as a characterization of the phase transition threshold of the sizes of multiple packings using general shapes in a finite product space.

\footnotetext{
${ }^{6}$ The word "achieve" will be formally defined in Definition 4.

${ }^{7}$ The condition "sufficiently large" here means larger than some constant independent of $n$. In our applications, the positive rate of a code is more than enough to certify the "sufficiently large" criterion.

${ }^{8}$ Given a list $\left(\underline{x}_{1}, \cdots, \underline{x}_{L}\right)$ of vectors in $\mathcal{X}^{n}$ (where $\mathcal{X}$ is a finite set), the empirical distribution, a.k.a. the type/histogram, of $\left(\underline{x}_{1}, \cdots, \underline{x}_{L}\right)$ is the distribution $P_{\mathbf{x}_{1}, \cdots, \mathbf{x}_{L}}$ defined as $P_{\mathbf{x}_{1}, \cdots, \mathbf{x}_{L}}\left(x_{1}, \cdots, x_{L}\right)=\frac{1}{n}\left|\left\{i \in[n]: \underline{x}_{1}(i)=x_{1}, \cdots, \underline{x}_{L}(i)=x_{L}\right\}\right|$ for all $\left(x_{1}, \cdots, x_{L}\right) \in \mathcal{X}^{L}$. See Definition 10
} 
Combining it with the hypergraph Turán's theorem (Theorem 17) allows us to prove Theorem 1 . The formal version of Theorem 1 is stated and proved in Lemma 18.

Completely positive (CP) distributions are joint distributions of tuples of random variables that can be written as a convex combination of tensor products of identical distributions, e.g., $P_{\mathbf{x}_{1}, \cdots, \mathbf{x}_{L}}=\sum_{i} \lambda_{i} P_{\mathbf{x}_{i}}^{\otimes L}$ for some coefficients $\left\{\lambda_{i}\right\}_{i}$ and distributions $\left\{P_{\mathbf{x}_{i}}\right\}_{i}$. The above theorem allows us to bound the the distribution of $\underline{\mathbf{s}}$ and to justify the validity of the previously described jamming strategy. This is because, CP distributions are invariant under permutations by definition, i.e., $P_{\mathbf{x}_{1}, \cdots, \mathbf{x}_{L}}=P_{\mathbf{x}_{\pi(1)}, \cdots, \mathbf{x}_{\pi(L)}}$ for any $\pi$. By Theorem 1 (with $L$ in the theorem being $L+1)$, the event that $\mathcal{L}^{\prime}=\left(\underline{\mathbf{x}}_{0}, \mathcal{L}\right)$ has empirical distribution approximately $P_{\mathbf{x}_{0}, \mathbf{x}_{1}, \cdots, \mathbf{x}_{L}}$ for some order- $(L+1)$ CP distribution $P_{\mathbf{x}_{0}, \mathbf{x}_{1}, \cdots, \mathbf{x}_{L}}$ happens with constant probability. Conditioned on this event, the distribution of $\underline{\mathbf{s}}_{\pi(\mathcal{L})}$ for any permutation $\pi$ is with high probability tightly concentrated around $\left[P_{\mathbf{x}_{0}, \mathbf{x}_{1}, \cdots, \mathbf{x}_{L}} U_{\mathbf{s} \mid \mathbf{x}_{1}, \cdots, \mathbf{x}_{L}}\right]_{\mathbf{s}}^{\otimes n_{9}}$. This is a product distribution independent of the particular realization of James's spoofing list $\mathcal{L}$ and the permutation $\pi$. The above argument hence basically justifies the effectiveness of the translation from symbol-wise list-symmetrizability to vector-wise list-symmetrizability. Indeed, even in the presence of state constraints, such a translation can be operationally realized by the previously described jamming strategy which we call CP-symmetrization. (See Section IX for the precise description of CP-symmetrization). The rest of the proof (which is deferred to Appendix B) can be finished using relatively "standard" techniques (with some careful tweaks) along the lines of [CN88b], [Hug97], [SG12].

The above argument shows that if a channel is $L$-CP-symmetrizable (see Definition 14 for the formal definition) then the $L$-list-decoding capacity is zero. That is, $L$-CP-symmetrizability is a sufficient condition of zero $L$ list-decoding capacity. We then sketch a matching achievability argument showing that this condition is also necessary. This turns out to be a relatively straightforward extension to the classical results by [CN88b], [Hug97], [SG12]. Specifically, by non-list-symmetrizability, James could not find a jamming channel $U_{\mathbf{s} \mid \mathbf{x}_{1}, \cdots, \mathbf{x}_{L}}$ satisfying the aforementioned property. This means for some CP distributions, no matter which $U_{\mathbf{s} \mid \mathbf{x}_{1}, \cdots, \mathbf{x}_{L}}$ James uses, the aforementioned jamming strategy fails since the jamming sequence $\underline{\mathbf{s}}_{\mathcal{L}}$ generated from a spoofing list $\mathcal{L}$ of a CP type $P_{\mathbf{x}_{1}, \cdots, \mathbf{x}_{L}}$ violates some state constraints. Alice could leverage that particular completely positive distribution $P_{\mathbf{x}_{1}, \cdots, \mathbf{x}_{L}}$ to construct a "good" code $\mathcal{C}$. By complete positivity, assume $P_{\mathbf{x}_{1}, \cdots, \mathbf{x}_{L}}$ can be decomposed as $\sum_{i=1}^{k} \lambda_{i} P_{\mathbf{x}_{i}}^{\otimes L}$ for some coefficients $\left\{\lambda_{i}\right\}_{i=1}^{k}$ and some distributions $\left\{P_{\mathbf{x}_{i}}\right\}_{i=1}^{k}$. Alice simply samples $|\mathcal{X}|^{n R}$ codewords for some constant $R \in(0,1]$ each of which is independently generated using the following distribution. To sample a codeword $\underline{\mathbf{x}}$, sample the first $n \lambda_{1}$ components independently from distribution $P_{\mathbf{x}_{1}}$, sample the next $n \lambda_{2}$ components independently from distribution $P_{\mathbf{x}_{2}}, \ldots$, sample the last $n \lambda_{k}$ components independently from distribution $P_{\mathbf{x}_{k}}$. By measure concentration, with high probability every size- $L$ list in $\mathcal{C}$ has joint type approximately $\sum_{i=1}^{k} \lambda_{i} P_{\mathbf{x}_{i}}^{\otimes L}$. Following the classical techniques by Csiszár and Narayan [CN88b], this further implies that with high probability such a random code is resilient to any feasible jamming strategy. This claim may not be immediately clear to readers who are not familiar with the AVC literature, since we are claiming the possibility of reliable communication robust to any jamming strategy, not necessarily of the form of CP-symmetrization introduced before. However, thanks to Csiszár and Narayan [CN88b], this claim does hold and the proof is nowadays standard.

\section{ORGANIZATION OF THIS PAPER}

The rest of the paper is organized as follows. The notational convention followed in this paper is introduced in Section V. Preliminaries on probability theory, oblivious AVC model, channel coding, list-decodable codes and information measures are given in Section VI. Formal presentation starts from Section VII onwards. The core definition of CP-symmetrizability is introduced in Section VII. Given this, formal statements of our main theorems are stated and compared with those in [SG12] in Section VIII. Technical proofs start from Section IX onwards. We describe and (partly) analyze CP-symmetrization, the most conceptually novel and technically challenging part of this work, in Section IX. Part of the proof is delegated to Appendices A and B. The above three sections jointly prove Theorem 12. We prove the capacity lower bound in Section X by designing and analyzing a coding scheme. Part of the proof is deferred to Appendices C and D. The above three sections jointly prove Theorem 13 . We prove, conditioned on Conjecture 21, a matching capacity upper bound in Section XI with part of the proof left for Appendix E. The above two sections jointly prove Theorem 14.

\footnotetext{
${ }^{9}$ Here the notation $[\cdot]_{\mathbf{s}}$ refers to the marginal on the variable $\mathbf{s}$ of the joint distribution in the bracket.
} 


\section{NOTATION}

Sets are denoted by capital letters in calligraphic typeface, e.g., $\mathcal{X}, \mathcal{S}, \mathcal{Y}$, etc. All alphabets in this paper are finite sized. For a positive integer $M$, we use $[M]$ to denote $\{1, \cdots, M\}$. Let $\mathcal{X}$ be a finite set. For an integer $k \leqslant|\mathcal{X}|$, we use $\left(\begin{array}{c}\mathcal{X} \\ k\end{array}\right)$ to denote $\left\{\mathcal{X}^{\prime} \subseteq \mathcal{X}:\left|\mathcal{X}^{\prime}\right|=k\right\}$. When we write $\left(\begin{array}{c}{[M]} \\ L\end{array}\right)$, we think of an element $\left\{i_{1}, \cdots, i_{L}\right\}$ in it as in ascending order, i.e., $i_{1}<\cdots<i_{L}$. Similarly, we define $\left(\begin{array}{c}\mathcal{X} \\ \leqslant k\end{array}\right):=\left\{\mathcal{X}^{\prime} \subseteq \mathcal{X}:\left|\mathcal{X}^{\prime}\right| \leqslant k\right\}$.

Random variables are denoted by lowercase letters in boldface, e.g., $\mathbf{x}, \mathbf{s}, \mathbf{y}$, etc. Their realizations are denoted by corresponding lowercase letters in plain typeface, e.g., $x, s, y$, etc. Vectors (random or fixed) of length $n$, where $n$ is the blocklength of the code without further specification, are denoted by lowercase letters with underlines, e.g., $\underline{\mathbf{x}}, \underline{\mathbf{s}}, \underline{\mathbf{y}}, \underline{x}, \underline{s}, \underline{y}$, etc. The $i$-th entry of a vector $\underline{x} \in \mathcal{X}^{n}\left(\operatorname{resp} . \underline{\mathbf{x}} \in \mathcal{X}^{n}\right)$ is denoted by $\underline{x}(i)$ (resp. $\underline{\mathbf{x}}(i)$ ). Let $\mathcal{L}=\left\{i_{1}, \cdots, i_{L}\right\}$ be a finite set of nonnegative integers such that $i_{1}<\cdots<i_{L}$. We use $x_{\mathcal{L}}$ (resp. $\mathbf{x}_{\mathcal{L}}$ ) to denote $\left(x_{i_{1}}, \cdots, x_{i_{L}}\right)$ (resp. $\left(\mathbf{x}_{i_{1}}, \cdots, \mathbf{x}_{i_{L}}\right)$ ). Matrices are denoted by capital letters in boldface, e.g., $\mathbf{A}, \mathbf{B}$, etc. Similarly, the $(i, j)$-th entry of a matrix $\mathbf{G} \in \mathbb{F}^{n \times m}$ is denoted by $\mathbf{G}(i, j)$. We sometimes write $\mathbf{G}_{n \times m}$ to explicitly specify its dimension. For square matrices, we write $\mathbf{G}_{n}$ for short. The letter $\mathbf{I}$ is reserved for the identity matrix.

We use the standard Bachmann-Landau (Big-Oh) notation. For $x \in \mathbb{R}$, let $[x]^{+}:=\max \{x, 0\}$. For two real-valued functions $f(n), g(n)$ of positive integers, we say that $f(n)$ asymptotically equals $g(n)$, denoted by $f(n)=g(n)$, if $\lim _{n \rightarrow \infty} f(n) / g(n)=1$. We write $f(n) \doteq g(n)($ read $f(n)$ dot equals $g(n))$ if $\lim _{n \rightarrow \infty}(\log f(n)) /(\log g(n))=1$. Note that $f(n)=g(n)$ implies $f(n) \doteq g(n)$, but the converse is not true. Related notations such as dot less/larger than (or equal to), denoted by $\dot{<} />$ (or $\leqslant / \geqslant$ ) can be similarly defined. For any $\mathcal{A} \subseteq \mathcal{X}$, the indicator function of $\mathcal{A}$ is defined as, for any $x \in \mathcal{X}$,

$$
\mathbb{1}_{\mathcal{A}}(x):=\left\{\begin{array}{ll}
1, & x \in \mathcal{A} \\
0, & x \notin \mathcal{A}
\end{array} .\right.
$$

At times, we will slightly abuse notation by saying that $\mathbb{1}\{\mathrm{A}\}$ is 1 when event $\mathrm{A}$ happens and 0 otherwise. Note that $\mathbb{1}_{\mathcal{A}}(\cdot)=\mathbb{1}\{\cdot \in \mathcal{A}\}$. In this paper, all logarithms are to the base 2 .

We use $\Delta(\mathcal{X})$ to denote the probability simplex on $\mathcal{X}$. Related notations such as $\Delta(\mathcal{X} \times \mathcal{Y})$ and $\Delta(\mathcal{Y} \mid \mathcal{X})$ are similarly defined. For a distribution $P_{\mathbf{x}, \mathbf{y} \mid \mathbf{u}} \in \Delta(\mathcal{X} \times \mathcal{Y} \mid \mathcal{U})$, we use $\left[P_{\mathbf{x}, \mathbf{y} \mid \mathbf{u}}\right]_{\mathbf{x} \mid \mathbf{u}} \in \Delta(\mathcal{X} \mid \mathcal{U})$ to denote the marginal distribution onto $\mathbf{x}$ given $\mathbf{u}$, i.e., for every $x \in \mathcal{X}, u \in \mathcal{U},\left[P_{\mathbf{x}, \mathbf{y} \mid \mathbf{u}}\right]_{\mathbf{x} \mid \mathbf{u}}(x \mid u)=\sum_{y \in \mathcal{Y}} P_{\mathbf{x}, \mathbf{y} \mid \mathbf{u}}(x, y \mid u)$. We use $\Delta^{(n)}(\mathcal{X})$ to denote the set of types (i.e., empirical distributions/histograms, see Definition 10 for formal definitions) of length- $n$ vectors over alphabet $\mathcal{X}$. That is, $\Delta^{(n)}(\mathcal{X})$ consists of all distributions $P_{\mathbf{x}} \in \Delta(\mathcal{X})$ that can be induced by $\mathcal{X}^{n}$-valued vectors. Other notations such as $\Delta^{(n)}(\mathcal{X} \times \mathcal{Y})$ and $\Delta^{(n)}(\mathcal{Y} \mid \mathcal{X})$ are similarly defined. The notation $\mathbf{x} \sim P_{\mathbf{x}}$ (resp. $\underline{\mathbf{x}} \sim P_{\underline{\mathbf{x}}}$ ) means that the p.m.f. of a random variable (resp. vector) $\mathbf{x}\left(\right.$ resp. $\underline{\mathbf{x}}$ ) is $P_{\mathbf{x}}$ (resp. $P_{\underline{\mathbf{x}}}$ ). If $\mathbf{x}$ is uniformly distributed in $\mathcal{X}$, then we write $\mathrm{x} \sim \mathcal{X}$. The symmetric group of degree $N \in \mathbb{Z}_{\geqslant 1}$ is denoted by $S_{N}$. It consists of all permutations, typically denoted by lowercase Greek letters, on a set of $N$ elements. When the set is a subset of nonnegative integers, we think of its elements as listed in ascending order. If $\mathcal{N}=\left\{i_{1}, \cdots, i_{N}\right\} \subset \mathbb{Z}_{\geqslant 1}$ such that $i_{1}<\cdots<i_{N}$, then for any $\pi \in S_{N}$, we define $\pi(\mathcal{N}):=\left(\pi\left(i_{1}\right), \cdots, \pi\left(i_{N}\right)\right)$. Throughout this paper, we use $d(\cdot, \cdot)$ to denote the $\ell^{1}$ distance between two distributions. Specifically, for $P, Q \in \Delta(\mathcal{X}), d(P, Q):=\sum_{x \in \mathcal{X}}|P(x)-Q(x)|$. For a subset $\mathcal{A} \subseteq \Delta(\mathcal{X})$, the distance between $P$ and $\mathcal{A}$ is defined as $d(P, \mathcal{A}):=\min _{Q \in \mathcal{A}} d(P, Q)$. The inner product between $P$ and $Q$ is defined as $\langle P, Q\rangle:=\sum_{x \in \mathcal{X}} P(x) Q(x)$. The $\ell^{p}$-norm of a vector is denoted by $\|\cdot\|_{p}$.

\section{PRELIMINARY}

\section{A. Probability}

Lemma 2 (Markov). If $X$ is a nonnegative random variable, then for any $a>0, \operatorname{Pr}[X \geqslant a] \leqslant \mathbb{E}[X] / a$.

Lemma 3 (Chebyshev). If $X$ is an integrable random variable with finite expectation and finite nonzero variance, then for any $a>0, \operatorname{Pr}[|X-\mathbb{E}[X]| \geqslant a] \leqslant \operatorname{Var}[X] / a^{2}$.

Lemma 4 (Sanov). Let $\mathcal{Q} \subset \Delta(\mathcal{X})$ be a subset of distributions such that it is equal to the closure of its interior. Let $\underline{\mathbf{x}} \sim P_{\mathbf{x}}^{\otimes n}$ for some $P_{\mathbf{x}} \in \Delta(\mathcal{X})$. Then

$$
\operatorname{Pr}\left[\tau_{\underline{\mathbf{x}}} \in \mathcal{Q}\right] \doteq 2^{-n \inf _{Q_{\mathbf{x}} \in \mathcal{Q}} D\left(Q_{\mathbf{x}} \| P_{\mathbf{x}}\right)},
$$

where the Kullback-Leibler divergence $D(\cdot \| \cdot)$ between two distributions is defined in Definition 9.

Lemma 5 (Pinsker). Let $P, Q \in \Delta(\mathcal{X})$. Then $D(P \| Q) \geqslant \frac{1}{2 \ln 2}\|P-Q\|_{1}^{2}$. 


\section{B. Oblivious AVCs and list-decoding}

We first formally define the oblivious adversarial channel model concerned with in this paper. Oblivious AVCs are communication channels governed by an oblivious adversary in the following sense. The adversary, referred to as James, aims to prevent reliable transmission from Alice to Bob from happening by injecting carefully designed noise to the channel. Importantly, we assume that an oblivious adversary does not get to see Alice's transmission (though he does know the codebook which is always assumed to be revealed to everyone before communication happens).

Definition 1 (Oblivious AVCs). An oblivious $\operatorname{AVC} \mathcal{A}_{\text {obli }}=\left(\mathcal{X}, \mathcal{S}, \mathcal{Y}, \lambda_{\mathbf{x}}, \lambda_{\mathbf{s}}, W_{\mathbf{y} \mid \mathbf{x}, \mathbf{s}}\right)$ consists of three alphabets $\mathcal{X}, \mathcal{S}, \mathcal{Y}$ for the input, jamming and output sequences, respectively; input constraints $\lambda_{\mathbf{x}} \subseteq \Delta(\mathcal{X})$ and state constraints $\lambda_{\mathbf{s}} \subseteq \Delta(\mathcal{S})$; and an adversarial channel $W_{\mathbf{y} \mid \mathbf{x}, \mathbf{s}}$ from Alice to Bob governed by James. To avoid peculiar behaviours, we assume that both $\lambda_{\mathbf{x}}$ and $\lambda_{\mathbf{s}}$ are convex.

Codewords of types from $\lambda_{\mathrm{x}}$ are allowed to be input to the channel. Knowing the codebook $\mathcal{C}$, Alice's encoder $\phi$ and Bob's decoder $\psi$ (both of which are formally defined in Definition 2), James generates a jamming sequence $\underline{\mathbf{s}}$ and sends it through the channel $W_{\mathbf{y} \mid \mathbf{x}, \mathbf{s}}$. The channel generates $\underline{\mathbf{y}}$ in a memoryless manner: $\operatorname{Pr}[\underline{\mathbf{y}}=\underline{y} \mid \underline{\mathbf{x}}=\underline{x}, \underline{\mathbf{s}}=\underline{s}]=$ $W_{\mathbf{y} \mid \mathbf{x}, \mathbf{s}}^{\otimes n}(\underline{y} \mid \underline{x}, \underline{s})=\prod_{i=1}^{n} W_{\mathbf{y} \mid \mathbf{x}, \mathbf{s}}(\underline{y}(i) \mid \underline{x}(i), \underline{s}(i))$. The channel output $\underline{\mathbf{y}}$ is then received by Bob. See Figure 3 for a block diagram of the oblivious AVC model under list-decoding (see Definition 2 for the definition of listdecodability).

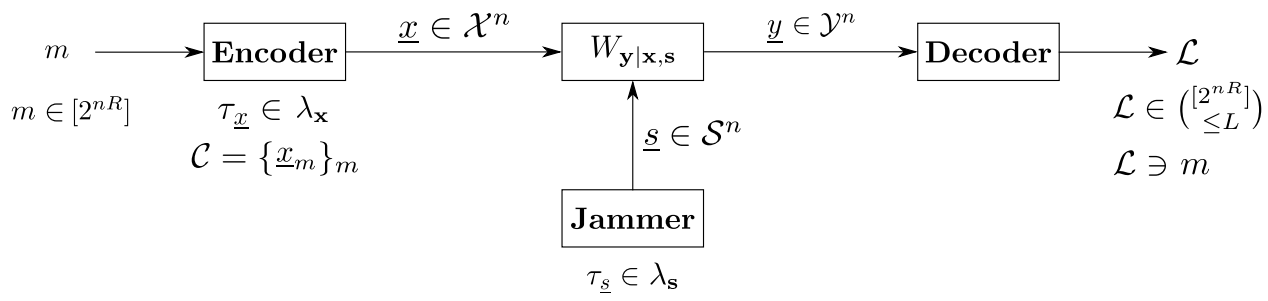

Fig. 3: Block diagram of an oblivious AVC under list-decoding.

Remark 2. Though the channel law is a product distribution, the jamming sequence may not follow a product distribution. Indeed, they do not necessarily follow any fixed distribution. This makes robust communication against adversaries challenging.

Remark 3. We have already seen an example of oblivious AVC — the oblivious bitflip channel — in Section II-A.

Definition 2 ( $L$-list-decodable codes for oblivious AVCs). An $L$-list-decodable code $\mathcal{C}$ for an oblivious AVC

$$
\mathcal{A}_{\mathrm{obli}}=\left(\mathcal{X}, \mathcal{S}, \mathcal{Y}, \lambda_{\mathbf{x}}, \lambda_{\mathbf{s}}, W_{\mathbf{y} \mid \mathbf{x}, \mathbf{s}}\right)
$$

consists of an encoder $\phi:[M] \rightarrow \mathcal{X}^{n}$ mapping any $m \in[M]$ to $\phi(m)=\underline{x}_{m}$ satisfying $\tau_{\underline{x}_{m}} \in \lambda_{\mathbf{x}}$; and an $L$-listdecoder $\psi: \mathcal{Y}^{n} \rightarrow\left(\begin{array}{c}{[M]} \\ \leqslant L\end{array}\right)$ such that $|\psi(\underline{y})| \leqslant L$ for all $\underline{y} \in \mathcal{Y}^{n}$. We call the image of $\phi$ the codebook, denoted by the same symbol $\mathcal{C}$ (or simply a code, with slight abuse of terminology), i.e., $\mathcal{C}:=\left\{\underline{x}_{i}\right\}_{i \in[M]}$. The length $n$ of each codeword is called the blocklength of $\mathcal{C}$. The rate of $\mathcal{C}$ is defined as $R(\mathcal{C}):=\frac{\log M}{n \log |\mathcal{X}|}$.

Remark 4. In this paper, when we talk about "a code", we always mean a sequence of codes of increasing blocklengths, i.e., $\left\{\mathcal{C}_{i}\right\}_{i \geqslant 1}$ each of blocklength $n_{i}$, where $n_{1}<n_{2}<\cdots \in \mathbb{Z}_{\geqslant 1}$ is an infinite sequence of increasing integers.

Definition 3 (Average probability of error). The average probability of L-list-decoding error of a code $\mathcal{C}$ when used over an oblivious channel $\mathcal{A}_{\text {obli }}$ is defined as

$$
P_{\mathrm{e}, \operatorname{avg}}(\mathcal{C}):=\max _{\underline{s} \in \mathcal{S}^{n}} \frac{1}{M} \sum_{i \in[M]} \operatorname{Pr}[\psi(\underline{\mathbf{y}}) \nexists i \mid \mathbf{i}=i, \underline{\mathbf{s}}=\underline{s}]=\max _{\underline{s} \in \mathcal{S}^{n}} \frac{1}{M} \sum_{i \in[M]} \sum_{\underline{y} \in \mathcal{Y}^{n}: \psi(\underline{y}) \nexists i} W_{\mathbf{y} \mid \mathbf{x}, \mathbf{s}}^{\otimes n}\left(\underline{y} \mid \underline{x}_{i}, \underline{s}\right) .
$$

Definition 4 (Achievable rates and $L$-list-decoding capacity). A rate $R$ is said to be achievable for an oblivious AVC $\mathcal{A}_{\text {obli }}$ under $L$-list-decoding if there is an infinite sequence of $L$-list-decodable codes $\left\{\mathcal{C}_{i}\right\}_{i \geqslant 1}$ for $\mathcal{A}_{\text {obli }}$ of 
increasing blocklengths such that $R\left(\mathcal{C}_{i}\right) \geqslant R$ for all $i$ and $P_{\mathrm{e}, \text { avg }}\left(\mathcal{C}_{i}\right) \stackrel{i \rightarrow \infty}{\longrightarrow} 0$. The supremum of all achievable rates is called the L-list-decoding capacity of $\mathcal{A}_{\text {obli }}$, denoted by $C_{L}\left(\mathcal{A}_{\text {obli }}\right)$.

Definition 5 (List-symmetrizability). For any oblivious $\operatorname{AVC} \mathcal{A}_{\text {obli }}=\left(\mathcal{X}, \mathcal{S}, \mathcal{Y}, \lambda_{\mathbf{x}}, \lambda_{\mathbf{s}}, W_{\mathbf{y} \mid \mathbf{x}, \mathbf{s}}\right)$, define the listsymmetrizability of $\mathcal{A}_{\mathrm{obli}}$, denoted by $L^{*}$, as the minimum $L$ such that $C_{L}\left(\mathcal{A}_{\mathrm{obli}}\right)>0$.

\section{Approximate constant-composition codes}

Definition 6 (Approximate constant-composition codes). A code $\mathcal{C} \subseteq \mathcal{X}^{n}$ is said to be $\left(\lambda, P_{\mathbf{x}}\right)$-constant-composition for some $P_{\mathbf{x}} \in \Delta(\mathcal{X})$ if every codeword $\underline{x} \in \mathcal{C}$ has type $\tau_{\underline{x}}$ satisfying $d\left(\tau_{\underline{x}}, P_{\mathbf{x}}\right) \leqslant \lambda$.

Definition 7 (Approximate constant-composition codes with time-sharing). A code $\mathcal{C}$ is said to be $\left(\lambda, \underline{u}, P_{\mathbf{x} \mid \mathbf{u}}\right)$ constant-composition for some $\underline{u} \in \mathcal{U}^{n}$ and some $P_{\mathbf{x} \mid \mathbf{u}} \in \Delta(\mathcal{X} \mid \mathcal{U})$ if every codeword $\underline{x} \in \mathcal{C}$ satisfies $d\left(\tau_{\underline{u}, \underline{x}}, \tau_{\underline{u}} P_{\mathbf{x} \mid \mathbf{u}}\right) \leqslant$ $\lambda$.

Definition 8 (Quantization/net). Given a metric space $(\mathcal{X}$, dist) and a constant $\eta>0$, an $\eta$-net or an $\eta$-quantization $\mathcal{N}$ of $\mathcal{X}$ w.r.t. the metric dist is a subset $\mathcal{N} \subset \mathcal{X}$ satisfying: for every $x \in \mathcal{X}$, there is an $x^{\prime} \in \mathcal{N}$ such that $\operatorname{dist}\left(x, x^{\prime}\right) \leqslant \eta$.

Taking a simple coordinate-wise quantization allows us to get an upper bound on the size of a net of a probability simplex. A proof of the following lemma can be found in [ZBJ20].

Lemma 6 (Bounds on the size of nets). Let $\mathcal{X}$ be a finite set. For any constant $\eta>0$, there is an $\eta$-net of $(\Delta(\mathcal{X}), d)$ of size at most $\left\lceil\left.\frac{|\mathcal{X}|}{2 \eta}\right|^{|\mathcal{X}|} \leqslant\left(\frac{|\mathcal{X}|}{2 \eta}+1\right)^{|\mathcal{X}|}\right.$.

A straightforward quantization argument allows us to reduce a general code to an approximate constant-composition code with only a constant multiplicative factor loss in the code size.

Lemma 7 (Approximate constant-composition reduction). For any $\lambda \in(0,1)$, any code $\mathcal{C} \subseteq \mathcal{X}^{n}$ contains $a\left(\lambda, P_{\mathbf{x}}\right)$ constant-composition subcode $\mathcal{C}^{\prime} \subseteq \mathcal{C}$ for some $P_{\mathbf{x}} \in \Delta(\mathcal{X})$ of size at least $|\mathcal{C}| / N$, where $N \leqslant\left(\frac{|\mathcal{X}|}{2 \lambda}+1\right)^{|\mathcal{X}|}$. In particular, $R(\mathcal{C}) \stackrel{n \rightarrow \infty}{=} R\left(\mathcal{C}^{\prime}\right)$.

\section{Information measures and method of types}

Definition 9 (Information measures). Let $\mathcal{X}, \mathcal{Y}$ be two finite sets and $P_{\mathbf{x}} \in \Delta(\mathcal{X})$. The Shannon entropy of $P_{\mathbf{x}}$ is defined as $H\left(P_{\mathbf{x}}\right):=\sum_{x \in \mathcal{X}} P_{\mathbf{x}}(x) \log \frac{1}{P_{\mathbf{x}}(x)}$. It is alternatively written as $H(\mathbf{x})$ where $\mathbf{x}$ is an $\mathcal{X}$-valued random variable whose p.m.f. is $P_{\mathbf{x}}$.

Let $\mathbf{y}$ be a $\mathcal{Y}$-valued random variable with joint p.m.f. $P_{\mathbf{x}, \mathbf{y}}$ with $\mathbf{x}$. The conditional entropy of $\mathbf{x}$ given $\mathbf{y}$ is defined as $H(\mathbf{x} \mid \mathbf{y}):=\sum_{(x, y) \in \mathcal{X} \times \mathcal{Y}} P_{\mathbf{x}, \mathbf{y}}(x, y) \log \frac{1}{P_{\mathbf{x} \mid \mathbf{y}}(x \mid y)}$. The mutual information between $\mathbf{x}$ and $\mathbf{y}$ is defined as $I(\mathbf{x} ; \mathbf{y}):=\sum_{(x, y) \in \mathcal{X} \times \mathcal{Y}} P_{\mathbf{x}, \mathbf{y}}(x, y) \log \frac{P_{\mathbf{x}, \mathbf{y}}(x, y)}{P_{\mathbf{x}}(x) P_{\mathbf{y}}(y)}$.

If $\mathbf{x}$ and $\mathbf{y}$ together with some $\mathcal{Z}$-valued random variable $\mathbf{z}$ have joint distribution $P_{\mathbf{x}, \mathbf{y}, \mathbf{z}}$, then the conditional mutual information between $\mathbf{x}$ and $\mathbf{y}$ given $\mathbf{z}$ is defined as

$$
I(\mathbf{x} ; \mathbf{y} \mid \mathbf{z}):=\sum_{z \in \mathcal{Z}} P_{\mathbf{z}}(z) \sum_{(x, y) \in \mathcal{X} \times \mathcal{Y}} P_{\mathbf{x}, \mathbf{y} \mid \mathbf{z}}(x, y \mid z) \log \frac{P_{\mathbf{x}, \mathbf{y} \mid \mathbf{z}}(x, y \mid z)}{P_{\mathbf{x} \mid \mathbf{z}}(x \mid z) P_{\mathbf{y} \mid \mathbf{z}}(y \mid z)} .
$$

If $P, Q \in \Delta(\mathcal{X})$ and $P$ is absolutely continuous w.r.t. $Q$ (i.e., $\operatorname{supp}(P) \subseteq \operatorname{supp}(Q)$ ), then the Kullback-Leibler $(K L)$ divergence between $P$ and $Q$ is defined as $D(P \| Q):=\sum_{x \in \mathcal{X}} P(x) \log \frac{P(x)}{Q(x)}$.

Readers who are not familiar with the basics of information measures are encouraged to refer to, e.g., [CT91]. We list below several basic and well-known properties of information measures that will be frequently used throughout the paper.

Lemma 8 (Properties of information measures). The information measures defined in Definition 9 satisfy the following properties.

1) Entropy, mutual information, KL divergence and their conditional versions are all nonnegative. 
2) Conditioning reduces entropy: $H(\mathbf{x} \mid \mathbf{y}) \leqslant H(\mathbf{x})$.

3) Alternative definitions: $I(\mathbf{x} ; \mathbf{y} \mid \mathbf{z})=H(\mathbf{x} \mid \mathbf{z})-H(\mathbf{x} \mid \mathbf{z}, \mathbf{y}) ; I(\mathbf{x} ; \mathbf{y})=D\left(P_{\mathbf{x}, \mathbf{y}} \| P_{\mathbf{x}} P_{\mathbf{y}}\right)$.

4) Chain rule: $I\left(\mathbf{x} ; \mathbf{y}_{1}, \mathbf{y}_{2} \mid \mathbf{z}\right)=I\left(\mathbf{x} ; \mathbf{y}_{1} \mid \mathbf{z}\right)+I\left(\mathbf{x} ; \mathbf{y}_{2} \mid \mathbf{z}, \mathbf{y}_{1}\right)$.

Definition 10 (Types, joint types and conditional types). Let $\mathcal{X}$ be a finite set and $n \in \mathbb{Z}_{\geqslant 1}$. The type of a vector $\underline{x} \in \mathcal{X}^{n}$, denoted by $\tau_{\underline{x}} \in \Delta(\mathcal{X})$, is the empirical distribution/histogram of $\underline{x}$ defined as: for every $x \in \mathcal{X}$, $\tau_{\underline{x}}(x)=\frac{1}{n} \mid\left\{i \in[n]: \underline{x}(i)=x \bar{x} \mid\right.$. The set of all types of $\mathcal{X}^{n}$-valued vectors is denoted by $\Delta^{(n)}(\mathcal{X})$. Let $\mathcal{Y}$ be another finite set and $\underline{y} \in \mathcal{Y}^{n}$. The joint type $\tau_{\underline{x}, \underline{y}}$ (and $\Delta^{(n)}(\mathcal{X} \times \mathcal{Y})$ correspondingly) and the conditional type $\tau_{\underline{x}} \mid \underline{y}$ (and $\Delta^{(n)}(\mathcal{X} \mid \mathcal{Y})$ correspondingly) are defined in a similar manner. Furthermore, these definitions can be extended to tuples of vectors in the canonical way.

Lemma 9 (Size of typical sets). Let $\underline{x} \in \mathcal{X}^{n}$ and let $P_{\mathbf{y}, \mathbf{x}} \in \Delta(\mathcal{Y} \times \mathcal{X})$ be such that $\tau_{\underline{x}}=\left[P_{\mathbf{y}, \mathbf{x}}\right]_{\mathbf{x}}$. Define the $\varepsilon$-conditionally typical set $\mathcal{A}_{\underline{\mathbf{y}} \mid \underline{x}}^{\varepsilon}\left(P_{\mathbf{y}, \mathbf{x}}\right)$ of $\mathcal{Y}^{n}$-valued sequences given $\underline{x}$ w.r.t. $P_{\mathbf{y}, \mathbf{x}}$ as

$$
\mathcal{A}_{\underline{\mathbf{y}} \underline{x} \underline{x}}^{\varepsilon}\left(P_{\mathbf{y}, \mathbf{x}}\right):=\left\{\underline{y} \in \mathcal{Y}^{n}: \forall(y, x) \in \mathcal{Y} \times \mathcal{X}, \frac{\tau_{\underline{y}, \underline{x}}(y, x)}{P_{\mathbf{y}, \mathbf{x}}(y, x)} \in[1-\varepsilon, 1+\varepsilon]\right\} .
$$

Then $2^{n(H(\mathbf{y} \mid \mathbf{x})-f(\varepsilon))} \leqslant\left|\mathcal{A}_{\underline{\mathbf{y}} \mid \underline{x}}^{\varepsilon}\left(P_{\mathbf{y}, \mathbf{x}}\right)\right| \leqslant 2^{n(H(\mathbf{y} \mid \mathbf{x})+f(\varepsilon))}$ for some constant $f(\varepsilon)>0$ such that $f(\varepsilon) \stackrel{\varepsilon \rightarrow 0}{\longrightarrow} 0$.

Lemma 10. Fix a channel $W_{\mathbf{y} \mid \mathbf{x}} \in \Delta(\mathcal{Y} \mid \mathcal{X})$. Let $P_{\mathbf{x}, \mathbf{s}, \mathbf{y}} \in \Delta(\mathcal{X} \times \mathcal{S} \times \mathcal{Y})$ and $P_{\mathbf{x}, \mathbf{s}}:=\left[P_{\mathbf{x}, \mathbf{s}, \mathbf{y}}\right]_{\mathbf{x}, \mathbf{s}}$. Then for any $(\underline{x}, \underline{s}) \in \mathcal{X}^{n} \times \mathcal{S}^{n}$ such that $\tau_{\underline{x}, \underline{s}}=P_{\mathbf{x}, \mathbf{s}}$,

$$
\sum_{\underline{y} \in \mathcal{Y}^{n}: \tau_{\underline{x}, \underline{s}, \underline{\underline{x}}}=P_{\mathbf{x}, \mathbf{s}, \mathbf{y}}} W_{\mathbf{y} \mid \mathbf{x}}(\underline{y} \mid \underline{x}) \leqslant 2^{-n D\left(P_{\mathbf{x}, \mathbf{s}, \mathbf{y}} \| P_{\mathbf{x}, \mathbf{s}} W_{\mathbf{y} \mid \mathbf{x}}\right)} .
$$

\section{CP-SYMMETRIZABILITY: A REFINED NOTION OF SYMMETRIZABILITY}

In this section, we introduce the notion of CP-symmetrizability which is one of the core definitions of this work.

Consider an oblivious $\operatorname{AVC} \mathcal{A}_{\text {obli }}=\left(\mathcal{X}, \mathcal{S}, \mathcal{Y}, \lambda_{\mathbf{x}}, \lambda_{\mathbf{s}}, W_{\mathbf{y} \mid \mathbf{x}, \mathbf{s}}\right)$. Without loss of generality, we assume that $\lambda_{\mathbf{x}} \subseteq$ $\Delta(\mathcal{X})$ and $\lambda_{\mathrm{x}} \subseteq \Delta(\mathcal{S})$ are convex polytopes defined by a finite number of linear inequalities and/or equalities, respectively. Specifically, the input constraints are given by

$$
\lambda_{\mathbf{x}}:=\left\{P_{\mathbf{x}} \in \Delta(\mathcal{X}): \forall j \leqslant \alpha, \sum_{x \in \mathcal{X}} A_{j}(x) P_{\mathbf{x}}(x) \leqslant \Gamma_{j}\right\}=\left\{P_{\mathbf{x}} \in \Delta(\mathcal{X}): \mathbf{A} P_{\mathbf{x}} \leqslant \underline{\Gamma}\right\},
$$

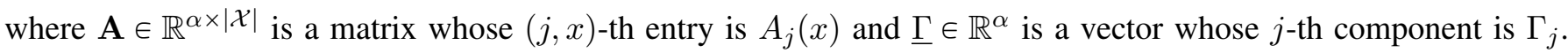
Similarly, the state constraints are

$$
\lambda_{\mathbf{s}}:=\left\{P_{\mathbf{s}} \in \Delta(\mathcal{S}): \forall j \leqslant \beta, \sum_{s \in \mathcal{S}} B_{j}(s) P_{\mathbf{s}}(s) \leqslant \Lambda_{j}\right\}=\left\{P_{\mathbf{s}} \in \Delta(\mathcal{S}): \mathbf{B} P_{\mathbf{s}} \leqslant \underline{\Lambda}\right\},
$$

where $\mathbf{B} \in \mathbb{R}^{\beta \times|\mathcal{S}|}$ is a matrix whose $(j, s)$-th entry is $B_{j}(s)$ and $\underline{\Lambda} \in \mathbb{R}^{\beta}$ is a vector whose $j$-th component is $\Lambda_{j}$. Define the corresponding sets of length- $n \mathcal{X}$-/S -sequences satisfying constraints $\lambda_{\mathbf{x}} / \lambda_{\mathbf{s}}$ as

$$
\Lambda_{\mathbf{X}}:=\left\{\underline{x} \in \mathcal{X}^{n}: \tau_{\underline{x}} \in \lambda_{\mathbf{x}}\right\}, \quad \Lambda_{\mathbf{S}}:=\left\{\underline{s} \in \mathcal{S}^{n}: \tau_{\underline{s}} \in \lambda_{\mathbf{s}}\right\} .
$$

To define CP-symmetrizability, we need several preliminary definitions.

Definition 11 (Obliviously $L$-symmetrizing distributions). Fix $L \in \mathbb{Z}_{\geqslant 1}$. Define the set of obliviously symmetrizing distributions as

$$
\mathcal{U}_{\text {obli, } L \text {-symm }}:=\left\{\begin{array}{c}
\quad \forall u \in \mathcal{U}, x_{0} \in \mathcal{X}, x_{[L]} \in \mathcal{X}^{L}, y \in \mathcal{Y}, \pi \in S_{L+1}, \\
U_{\mathbf{s} \mid \mathbf{u}, \mathbf{x}_{[L]} \in \Delta\left(\mathcal{S} \mid \mathcal{U} \times \mathcal{X}^{L}\right): \quad} \sum_{s \in \mathcal{S}} W_{\mathbf{y} \mid \mathbf{x}, \mathbf{s}}\left(y \mid x_{0}, s\right) U_{\mathbf{s} \mid \mathbf{u}, \mathbf{x}_{[L]}}\left(s \mid u, x_{[L]}\right) \\
=\sum_{s \in \mathcal{S}} W_{\mathbf{y} \mid \mathbf{x}, \mathbf{s}}\left(y \mid x_{\pi(0)}, s\right) U_{\mathbf{s} \mid \mathbf{u}, \mathbf{x}_{[L]}}\left(s \mid u, x_{\pi([L])}\right)
\end{array}\right\} .
$$

Remark 5. For technical reasons that will be clear momentarily, the symmetrizing distributions we need take the slightly more complicated form of $U_{\mathbf{s} \mid \mathbf{u}, \mathbf{x}_{1}, \cdots, \mathbf{x}_{L}}$ than $U_{\mathbf{s} \mid \mathbf{x}_{1}, \cdots, \mathbf{x}_{L}}$ mentioned in the overview section (Section III). One 
should think of $\mathbf{u}$ as a time-sharing variable used by Alice as part of the code design. We assume that the coding scheme, in particular $\mathbf{u}$, is also known to James. This is the reason why we allow the symmetrizing distribution to be conditioned on $\mathbf{u}$.

Definition 12 (Self-couplings). Let $P_{\mathbf{x}} \in \Delta(\mathcal{X})$ and $L \in \mathbb{Z}_{\geqslant 2}$. Define the set of order- $L P_{\mathbf{x}}$-self-couplings over $\mathcal{X}$ as

$$
\mathcal{J}^{\otimes L}\left(P_{\mathbf{x}}\right):=\left\{P_{\mathbf{x}_{1}, \cdots, \mathbf{x}_{L}} \in \Delta\left(\mathcal{X}^{L}\right): \forall i \in[L],\left[P_{\mathbf{x}_{1}, \cdots, \mathbf{x}_{L}}\right]_{\mathbf{x}_{i}}=P_{\mathbf{x}}\right\} .
$$

Definition 13 (Complete positivity (CP)). Let $P_{\mathbf{x}} \in \Delta(\mathcal{X})$ and $L \in \mathbb{Z}_{\geqslant 2}$. A distribution $P_{\mathbf{x}_{1}, \cdots, \mathbf{x}_{L}} \in \mathcal{J}^{\otimes L}\left(P_{\mathbf{x}}\right)$ is called $P_{\mathrm{x}}$-completely positive $(\mathrm{CP})$ if it can be written as a convex combination of tensor products of identical distributions. That is, there exist $k \in \mathbb{Z}_{>0}, \lambda_{1}, \cdots, \lambda_{k} \in[0,1]$ satisfying $\sum_{i=1}^{k} \lambda_{i}=1$, and $P_{\mathbf{x}_{1}}, \cdots, P_{\mathbf{x}_{k}} \in \Delta(\mathcal{X})$ such that

$$
P_{\mathbf{x}_{1}, \cdots, \mathbf{x}_{L}}=\sum_{i=1}^{k} \lambda_{i} P_{\mathbf{x}_{i}}^{\otimes L} .
$$

Or equivalently, there exist $k \in \mathbb{Z}_{>0}$, a time-sharing variable $\mathbf{u} \in \Delta(\mathcal{U})$ (where $\mathcal{U}:=[k]$ ) and a conditional distribution $P_{\mathbf{x} \mid \mathbf{u}} \in \Delta(\mathcal{X} \mid \mathcal{U})$ such that

$$
P_{\mathbf{x}_{1}, \cdots, \mathbf{x}_{L}}=\left[P_{\mathbf{u}} P_{\mathbf{x} \mid \mathbf{u}}^{\otimes L}\right]_{\mathbf{x}_{1}, \cdots, \mathbf{x}_{L}}
$$

The set of all order- $L P_{\mathbf{x}}$-completely positive distributions is denoted by $\mathrm{CP}^{\otimes L}\left(P_{\mathbf{x}}\right)$. For any $P_{\mathbf{x}_{1}, \cdots, \mathbf{x}_{L}} \in \mathrm{CP}^{\otimes L}\left(P_{\mathbf{x}}\right)$, we call a decomposition of the form Equation (1) or Equation (2) a CP-decomposition.

Remark 6. Since a $P_{\mathbf{x}}$-CP-distribution should be a $P_{\mathbf{x}}$-self-coupling in the first place, any $\left(P_{\mathbf{u}}, P_{\mathbf{x} \mid \mathbf{u}}\right)$ given by a CP-decomposition satisfies $\left[P_{\mathbf{u}} P_{\mathbf{x} \mid \mathbf{u}}\right]_{\mathbf{x}}=P_{\mathbf{x}}$.

Remark 7. The CP-decomposition of a CP-distribution is not necessarily unique. In particular, the number $k$ of components in a CP-decomposition (Equation (1)) of $P_{\mathbf{x}_{1}, \cdots, \mathbf{x}_{L}} \in \mathrm{CP}^{\otimes L}\left(P_{\mathbf{x}}\right)$ is not necessarily unique. Among all CP-decompositions, the smallest $k=|\mathcal{U}|$ given by a CP-decomposition is called the CP-rank, denoted by CP-rk, of $P_{\mathbf{x}_{1}, \cdots, \mathbf{x}_{k}} \in \mathrm{CP} \otimes L\left(P_{\mathbf{x}}\right)$. In fact, even if a CP-decomposition is required to contain the same number of terms as CP-rank, it still may not be unique.

Definition 14 (CP-symmetrizability). Fix an oblivious $\operatorname{AVC} \mathcal{A}_{\text {obli }}=\left(\mathcal{X}, \mathcal{S}, \mathcal{Y}, \lambda_{\mathbf{x}}, \lambda_{\mathbf{s}}, W_{\mathbf{y} \mid \mathbf{x}, \mathbf{s}}\right)$. For $L \in \mathbb{Z}_{\geqslant 1}$, an input distribution $P_{\mathbf{x}} \in \lambda_{\mathbf{x}}$ is said to be obliviously CP-L-symmetrizable (or $L$-symmetrizable for short) if for every $P_{\mathbf{x}_{1}, \cdots, \mathbf{x}_{L}} \in \mathrm{CP}^{\otimes L}\left(P_{\mathbf{x}}\right)$ and every its CP-decomposition $P_{\mathbf{x}_{1}, \cdots, \mathbf{x}_{L}}=\left[P_{\mathbf{u}} P_{\mathbf{x} \mid \mathbf{u}}\right]^{\otimes L}$ where $P_{\mathbf{u}} \in \Delta(\mathcal{U}), P_{\mathbf{x} \mid \mathbf{u}} \in$ $\Delta(\mathcal{X} \mid \mathcal{U})$, there is a symmetrizing distribution $U_{\mathbf{s} \mid \mathbf{u}, \mathbf{x}_{1}, \cdots, \mathbf{x}_{L}} \in \mathcal{U}_{\mathrm{obli}, L \text {-symm }}$ such that

$$
P_{\mathbf{s}}=\left[P_{\mathbf{u}} P_{\mathbf{x} \mid \mathbf{u}}^{\otimes L} U_{\mathbf{s} \mid \mathbf{u}, \mathbf{x}_{1}, \cdots, \mathbf{x}_{L}}\right]_{\mathbf{s}} \in \lambda_{\mathbf{s}},
$$

or equivalently, for each $i \in[\beta]$, the "jamming cost" does not exceed the $i$-th constraint, i.e.,

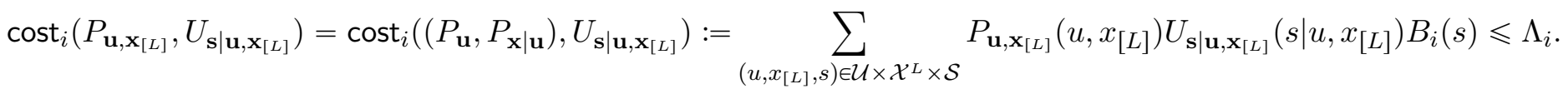

For an input distribution $P_{\mathbf{x}} \in \lambda_{\mathbf{x}}$, define the oblivious $P_{\mathbf{x}}$-CP-symmetrizability (or $P_{\mathbf{x}}$-symmetrizability for short) of $\mathcal{A}_{\text {obli }}$ as

$$
L_{\mathrm{CP}}^{*}\left(P_{\mathbf{x}}\right):=\max \left\{L \in \mathbb{Z}_{\geqslant 1}: P_{\mathbf{x}} \text { is obliviously CP- } L \text {-symmetrizable }\right\} .
$$

Define the oblivious CP-symmetrizability of $\mathcal{A}_{\mathrm{obli}}$ as $L_{\mathrm{CP}}^{*}:=\min _{P_{\mathbf{x}} \in \lambda_{\mathbf{x}}} L_{\mathrm{CP}}^{*}\left(P_{\mathbf{x}}\right)$.

Remark 8. With slight abuse of terminology, we interchangeably call $\left(P_{\mathbf{u}}, P_{\mathbf{x} \mid \mathbf{u}}\right)$ a CP-decomposition. We at times call $P_{\mathbf{x}_{1}, \cdots, \mathbf{x}_{L}} \in \mathrm{CP}^{\otimes L}\left(P_{\mathbf{x}}\right) L$-symmetrizable if for every CP-decomposition $\left(P_{\mathbf{u}}, P_{\mathbf{x} \mid \mathbf{u}}\right)$, Equation (3) or Equation (4)

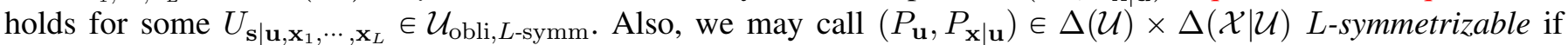
Equation (3) or Equation (4) holds for some $U_{\mathbf{s} \mid \mathbf{u}, \mathbf{x}_{1}, \cdots, \mathbf{x}_{L}} \in \mathcal{U}_{\mathrm{obli}, L \text {-symm. }}$ 
Remark 9. In the absence of constraints, Definition 14 collapses to Hughes's [Hug97] notion of list-symmetrizability. When $L=1$, Definition 14 collapses to Csiszár-Narayan's [CN88b] notion of symmetrizability. See Section XII-A for more details on this reduction. However, Definition 14 does not specialize to either the strong or weak listsymmetrizability (denoted by $L_{\text {strong }}^{*}$ and $L_{\text {weak }}^{*}$, respectively) due to Sarwate and Gastpar [SG12]. See below (Definition 15 and Definition 16) for the definition of $L_{\text {strong }}^{*}, L_{\text {weak }}^{*}$ and see Section VIII-A for a proper comparison of $L_{\mathrm{CP}}^{*}, L_{\text {strong }}^{*}$ and $L_{\text {weak }}^{*}$. In fact, one can find examples of oblivious AVCs for which the values of $L_{\mathrm{strong}}^{*}, L_{\mathrm{CP}}^{*}$ and $L_{\text {weak }}^{*}$ are strictly different. We will do this by developing a machinery that we call canonical constructions of oblivious channels. Taking proper canonical constructions allows us to show that there are channels for which $L_{\text {strong }}^{*}<L_{\mathrm{CP}}^{*}$ and there are channels for which $L_{\mathrm{CP}}^{*}<L_{\text {weak }}^{*}$. These constructions and their analysis will be presented in Section XIII.

In [SG12], the authors defined two notions of list-symmetrizability, known as the strong and weak list-symmetrizability. They were used for giving outer and inner bounds, respectively, on the $L$-list-decoding capacity of oblivious AVCs. Their definitions read as follows. Weak symmetrizability of an input distribution $P_{\mathbf{x}}$ replaces the quantifier " $\forall P_{\mathbf{x}_{1}, \cdots, \mathbf{x}_{L}} \in \mathrm{CP}^{\otimes L}\left(P_{\mathbf{x}}\right)$ " by a fixed distribution $P_{\mathbf{x}}^{\otimes L}$. Strong symmetrizability instead replaces that by all $P_{\mathbf{x}^{-}}$ self-couplings, i.e., the quantifier becomes " $\forall P_{\mathbf{x}_{1}, \cdots, \mathbf{x}_{L}} \in \mathcal{J}^{\otimes L}\left(P_{\mathbf{x}}\right)$ ". Apparently, for any $L \in \mathbb{Z}_{\geqslant 1}$, if $P_{\mathbf{x}}$ is strongly $L$-symmetrizable, then it is CP- $L$-symmetrizable; if $P_{\mathbf{x}}$ is CP- $L$-symmetrizable, then it is weakly $L$-symmetrizable. The notions of $L_{\text {strong }}^{*}$ and $L_{\text {weak }}^{*}$ are defined in the same way. We have the obvious relation $L_{\text {strong }}^{*} \leqslant L_{\mathrm{CP}}^{*} \leqslant L_{\text {weak }}^{*}$.

We state below the formal definitions of strong and weak list-symmetrizability for completeness.

Definition 15 (Strong symmetrizability). Fix an oblivious $\operatorname{AVC} \mathcal{A}_{\text {obli }}=\left(\mathcal{X}, \mathcal{S}, \mathcal{Y}, \lambda_{\mathbf{x}}, \lambda_{\mathbf{s}}, W_{\mathbf{y} \mid \mathbf{x}, \mathbf{s}}\right)$. For $L \in \mathbb{Z} \geqslant 1$, an input distribution $P_{\mathbf{x}} \in \lambda_{\mathbf{x}}$ is said to be obliviously strongly L-symmetrizable if for every $P_{\mathbf{x}_{[L]}} \in \mathcal{J}^{\otimes L}\left(P_{\mathbf{x}}\right)$, there is a symmetrizing distribution $U_{\mathbf{s} \mid \mathbf{x}_{[L]}} \in \mathcal{U}_{\mathrm{obli}, L \text {-symm }}{ }^{10}$ such that $\left[P_{\mathbf{x}_{[L]}} U_{\mathbf{s} \mid \mathbf{x}_{[L]}}\right]_{\mathbf{S}} \in \lambda_{\mathbf{s}}$. The oblivious strong symmetrizability of $\mathcal{A}_{\text {obli }}$ is defined as $L_{\text {strong }}^{*}:=\min _{P_{\mathbf{x}} \in \lambda_{\mathbf{x}}} \max \left\{L \in \mathbb{Z}_{\geqslant 1}: P_{\mathbf{x}}\right.$ is obliviously strongly $L$-symmetrizable $\}$.

Definition 16 (Weak symmetrizability). Fix an oblivious $\operatorname{AVC} \mathcal{A}_{\text {obli }}=\left(\mathcal{X}, \mathcal{S}, \mathcal{Y}, \lambda_{\mathbf{x}}, \lambda_{\mathbf{s}}, W_{\mathbf{y} \mid \mathbf{x}, \mathbf{s}}\right)$. For $L \in \mathbb{Z}_{\geqslant 1}$, an input distribution $P_{\mathbf{x}} \in \lambda_{\mathbf{x}}$ is said to be obliviously weakly L-symmetrizable if there is a symmetrizing distribution

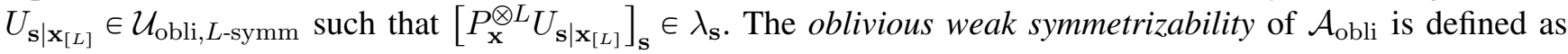

$$
L_{\text {weak }}^{*}:=\min _{P_{\mathbf{x}} \in \lambda_{\mathbf{x}}} \max \left\{L \in \mathbb{Z}_{\geqslant 1}: P_{\mathbf{x}} \text { is obliviously weakly } L \text {-symmetrizable }\right\} \text {. }
$$

\section{OUR RESULTS}

In this section, we give formal statements of our results and compare them in details with the closely related work by Sarwate and Gastpar [SG12].

Definition 17 (Capacity expression). Let $\mathcal{A}_{\mathrm{obli}}=\left(\mathcal{X}, \mathcal{S}, \mathcal{Y}, \lambda_{\mathbf{x}}, \lambda_{\mathbf{s}}, W_{\mathbf{y} \mid \mathbf{x}, \mathbf{s}}\right)$ be an oblivious AVC. Let $L \geqslant 1$ be a certain list-size. Define

$$
C_{L}=\max _{P_{\mathbf{x}} \in \lambda_{\mathbf{x}}: P_{\mathbf{x}}}{\min -L \text {-symmetrizable } U_{\mathbf{s} \mid \mathbf{u}} \in \Delta(\mathcal{S} \mid \mathcal{U}):\left[P_{\mathbf{u}} U_{\mathbf{s} \mid \mathbf{u}}\right]_{\mathbf{s}} \in \lambda_{\mathbf{s}}} I(\mathbf{x} ; \mathbf{y} \mid \mathbf{u}) .
$$

Here the maximization is taken over all feasible input distributions $P_{\mathbf{x}}$ that are non- $L$-symmetrizable. By Definition 14, each such $P_{\mathbf{x}}$ induces at least one non- $L$-symmetrizable CP-distribution $P_{\mathbf{x}_{1}, \cdots, \mathbf{x}_{L}}$. By Definition 13, each non- $L$-symmetrizable CP-distribution $P_{\mathbf{x}_{1}, \cdots, \mathbf{x}_{L}}$ further induces a non- $L$-symmetrizable pair $\left(P_{\mathbf{x}}, P_{\mathbf{x} \mid \mathbf{u}}\right)$ through Equation (2). Therefore for each $P_{\mathbf{x}} \in \lambda_{\mathbf{x}}$, the maximization is also implicitly taken over non- $L$-symmetrizable CPdistributions $P_{\mathbf{x}_{1}, \cdots, \mathbf{x}_{L}}$ and a pair $\left(P_{\mathbf{x}}, P_{\mathbf{x} \mid \mathbf{u}}\right)$ induced by a non- $L$-symmetrizable CP-decomposition of $P_{\mathbf{x}_{1}, \cdots, \mathbf{x}_{L}}$. In the minimization, the distribution $P_{\mathbf{u}}$ is the one given by the non- $L$-symmetrizable CP-decomposition in the maximization. The mutual information is evaluated using the joint distribution

$$
P_{\mathbf{u}, \mathbf{x}, \mathbf{y}}=\left[P_{\mathbf{u}} P_{\mathbf{x} \mid \mathbf{u}} U_{\mathbf{s} \mid \mathbf{u}} W_{\mathbf{y} \mid \mathbf{x}, \mathbf{s}}\right]_{\mathbf{u}, \mathbf{x}, \mathbf{y}},
$$

where $\left(P_{\mathbf{u}}, P_{\mathbf{x} \mid \mathbf{u}}\right)$ is the CP-decomposition in the maximization.

\footnotetext{
${ }^{10}$ Here and later in Definition 16, since there is no CP-distributions, the time-sharing variable $\mathbf{u}$ is absent. Hence the definition of $\mathcal{U}_{\text {obli, } L \text {-symm }}$ should be slightly changed to be the set of distributions $U_{\mathbf{s} \mid \mathbf{x}_{[L]}} \in \Delta\left(\mathcal{S} \mid \mathcal{X}^{L}\right)$ satisfying a certain system of identities.
} 
Remark 10. Note that the minimization is over distributions of the form $U_{\mathbf{s} \mid \mathbf{u}}$ such that the jamming distribution $P_{\mathbf{s}}$ satisfies the state constraints. Operationally, this corresponds to James jamming the channel using a noise sequence $\underline{\mathbf{s}}$ sampled from $U_{\mathbf{s} \mid \mathbf{u}}^{\otimes n}$. Intuitively, given the definition of $L$-symmetrizability (Definition 14), it might seem more natural to let James minimize the mutual information over distributions of the form $U_{\mathbf{s} \mid \mathbf{u}, \mathbf{x}_{[L]}}$ such that $\left[P_{\mathbf{u}} P_{\mathbf{x}}^{\otimes L} U_{\mathbf{s} \mid \mathbf{u}, \mathbf{x}_{[L]}}\right]_{\mathbf{s}} \in \lambda_{\mathbf{s}}$. Operationally, this corresponds to James first sampling a list $\left(\underline{\mathbf{x}}_{i_{1}}, \cdots, \underline{\mathbf{x}}_{i_{L}}\right)$ of $L$

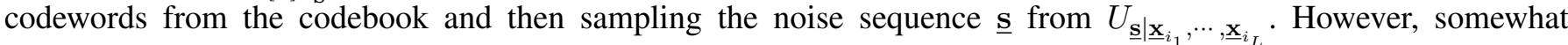
counterintuitively, we show in Section XII-C that the value of the resulting capacity expression under such a minimization is no smaller than Equation (5). That is, this seemingly more natural jamming strategy does not give a better converse bound. Hence, for the purpose of upper bounding the value of the capacity (whenever it is positive), we may restrict James to the less general type of jamming distributions $U_{\mathbf{s} \mid \mathbf{u}}$. To avoid confusion, we stress that: when James would like to test the positivity of the capacity, he does employ jamming distributions of $U_{\mathbf{s} \mid \mathbf{u}, \mathbf{x}_{[L]}}$ kind. Indeed, this is basically the CP-symmetrization attack that will be described and (partly) analyzed in Section IX.

The main results of this paper read as follows.

Theorem 11 (List-decoding capacity of general oblivious AVCs). Let $\mathcal{A}_{\mathrm{obli}}=\left(\mathcal{X}, \mathcal{S}, \mathcal{Y}, \lambda_{\mathbf{x}}, \lambda_{\mathbf{s}}, W_{\mathbf{y} \mid \mathbf{x}, \mathbf{s}}\right)$ be an oblivious AVC. Let $L \in \mathbb{Z}_{\geqslant 1}$ be the list-size. Let $C_{L}\left(\mathcal{A}_{\mathrm{obli}}\right)$ denote the L-list-decoding capacity of $\mathcal{A}_{\mathrm{obli}}$. Conditioned on Conjecture 21, we have the following capacity results. If $L>L_{\mathrm{CP}}^{*}$, then $C_{L}\left(\mathcal{A}_{\mathrm{obli}}\right)=C_{L}$ where $L_{\mathrm{CP}}^{*}$ and $C_{L}$ were defined in Definition 14 and Definition 17, respectively. If $L \leqslant L_{\mathrm{CP}}^{*}$, then $C_{L}\left(\mathcal{A}_{\mathrm{obli}}\right)=0$.

More specifically, we prove the following three theorems.

Theorem 12 (Converse: CP-symmetrization). Fix an oblivious $A V C \mathcal{A}_{\mathrm{obli}}=\left(\mathcal{X}, \mathcal{S}, \mathcal{Y}, \lambda_{\mathbf{x}}, \lambda_{\mathbf{s}}, W_{\mathbf{y} \mid \mathbf{x}, \mathbf{s}}\right)$ and a list-size $L \in \mathbb{Z}_{\geqslant 1}$. If $L \leqslant L_{\mathrm{CP}}^{*}$, then $C_{L}\left(\mathcal{A}_{\mathrm{obli}}\right)=0$. That is, there is a jamming strategy and a constant $c_{1}>0$ such that under this jamming strategy, any code $\mathcal{C}$ satisfying input constraints of rate $R(\mathcal{C})>0$ has average probability of error $P_{\mathrm{e}, \mathrm{avg}}(\mathcal{C}) \geqslant c_{1}$.

Proof. See Section IX.

Theorem 13 (Achievability). Fix an oblivious $A V C \mathcal{A}_{\mathrm{obli}}=\left(\mathcal{X}, \mathcal{S}, \mathcal{Y}, \lambda_{\mathbf{x}}, \lambda_{\mathbf{s}}, W_{\mathbf{y} \mid \mathbf{x}, \mathbf{s}}\right)$ and a list-size $L \in \mathbb{Z}_{\geqslant 1}$. If $L>L_{\mathrm{CP}}^{*}$, then $C_{L}\left(\mathcal{A}_{\mathrm{obli}}\right) \geqslant C_{L}$ where $C_{L}$ was defined in Equation (5). That is, for any $\delta>0$, there is a distribution over input-feasible codes of rate $R \leqslant C_{L}-\delta$ such that, with high probability, a random code $\mathcal{C}$ sampled from this ensemble has average probability of error $P_{\mathrm{e}, \mathrm{avg}}(\mathcal{C})=o(1)$.

Proof. See Section X.

Theorem 14 (Converse: capacity upper bound). Fix an oblivious $A V C \mathcal{A}_{\mathrm{obli}}=\left(\mathcal{X}, \mathcal{S}, \mathcal{Y}, \lambda_{\mathbf{x}}, \lambda_{\mathbf{s}}, W_{\mathbf{y} \mid \mathbf{x}, \mathbf{s}}\right)$ and a list-size $L \in \mathbb{Z}_{\geqslant 1}$. If $L>L_{\mathrm{CP}}^{*}$, then conditioned on Conjecture $21, C_{L}\left(\mathcal{A}_{\mathrm{obli}}\right) \geqslant C_{L}$ where $C_{L}$ was defined in Equation (5). That is, for any $\delta>0$, there is a jamming strategy and a constant $c_{2}>0$ such that under this jamming strategy, any code $\mathcal{C}$ satisfying input constraints of rate $R(\mathcal{C}) \geqslant C_{L}+\delta$ has average probability $P_{\mathrm{e}, \text { avg }}(\mathcal{C}) \geqslant c_{2}$.

Proof. See Section XI.

\section{A. Comparison of our results with [SG12]}

Using the notions of strong (Definition 15) and weak (Definition 16) symmetrizability mentioned in Section VII, Sarwate and Gastpar [SG12] proved upper and lower bounds, respectively, on the $L$-list-decoding capacity. Their upper and lower bounds read as follows:

$$
\begin{aligned}
\check{C}_{L}:=\max _{\substack{P_{\mathbf{x}} \in \lambda_{\mathbf{x}}: \\
P_{\mathbf{x}} \text { non-strongly-L-symmetrizable }}} \min _{U_{\mathbf{s}} \in \lambda_{\mathbf{s}}} I(\mathbf{x} ; \mathbf{y}), \\
\widehat{C}_{L}:=\max _{\substack{P_{\mathbf{x}} \in \lambda_{\mathbf{x}}: \\
P_{\mathbf{x}} \text { non-weakly- } L \text {-symmetrizable }}} \min _{U_{\mathbf{s}} \in \lambda_{\mathrm{s}}} I(\mathbf{x} ; \mathbf{y}),
\end{aligned}
$$

where the mutual information terms are evaluated according to $P_{\mathbf{x}, \mathbf{y}}=\left[P_{\mathbf{x}} U_{\mathbf{s}} W_{\mathbf{y} \mid \mathbf{x}, \mathbf{s}}\right]_{\mathbf{x}, \mathbf{y}}$. 
Theorem 15. Let $\mathcal{A}_{\mathrm{obli}}$ be an oblivious AVC. Let $L \in \mathbb{Z}_{\geqslant 1}$ be the list-size. Then the L-list-decoding capacity $C_{L}\left(\mathcal{A}_{\mathrm{obli}}\right)$ of $\mathcal{A}_{\mathrm{obli}}$ satisfies the following.

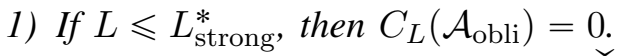

2) If $L>L_{\text {strong, then }}^{*} C_{L}\left(\mathcal{A}_{\text {obli }}\right) \leqslant \check{C}_{L}$.

3) If $L>L_{\text {weak }}^{*}$, then $C_{L}\left(\mathcal{A}_{\text {obli }}\right) \geqslant \widehat{C}_{L}$.

It was open before this work whether positive rate is achievable for $L_{\text {strong }}^{*}<L \leqslant L_{\text {weak }}^{*}$.

A visual comparison of our results with those in [SG12] is shown in Figure 4. More specifically, comparisons of

achievability in [SG12]

achievability in this work

rate upper bound in [SG12]

rate upper bound in this work

symmetrization converse in [SG12]

symmetrization converse in this work

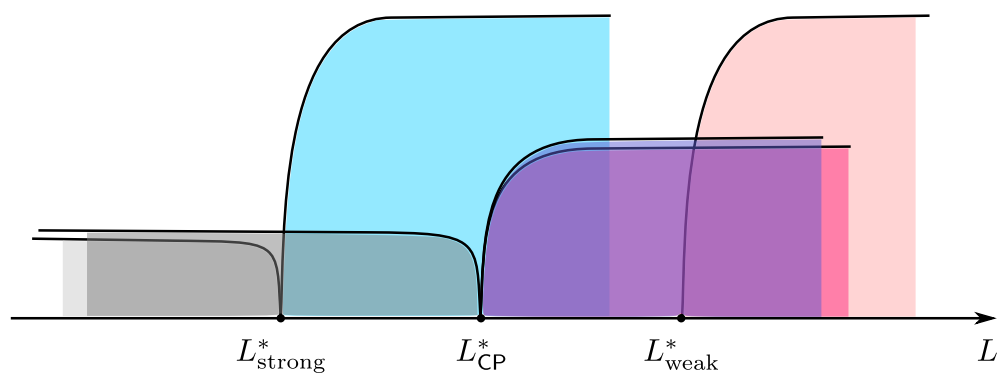

Fig. 4: A visual comparison of results in this paper with those in [SG12].

achievability, symmetrization converse and capacity upper bounds are shown in Figure 5a, Figure 5b and Figure 5c, respectively.

For $L$ in different ranges, we align our results with [SG12]'s for the readers' convenience.

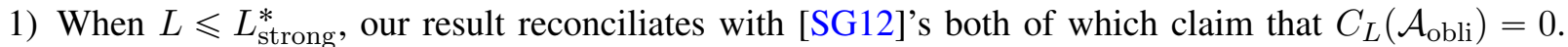

2) When $L_{\text {strong }}^{*}<L \leqslant L_{\mathrm{CP}}^{*}$, our symmetrization result claims that $C_{L}\left(\mathcal{A}_{\mathrm{obli}}\right)=0$, improving on [SG12]'s upper bound $\breve{C}_{L}$.

3) When $L>L_{\mathrm{CP}}^{*}$, our upper bound (conditioned on Conjecture 21) $C_{L}$ improves on $\check{C}_{L}$. Indeed,

$$
\begin{aligned}
I(\mathbf{x} ; \mathbf{y} \mid \mathbf{u}) & =H(\mathbf{y} \mid \mathbf{u})-H(\mathbf{y} \mid \mathbf{x}, \mathbf{u}) \\
& =H(\mathbf{y} \mid \mathbf{u})-H(\mathbf{y} \mid \mathbf{x}) \\
& \leqslant H(\mathbf{y})-H(\mathbf{y} \mid \mathbf{x}) \\
& =I(\mathbf{x} ; \mathbf{y}) .
\end{aligned}
$$

Equation (6) follows since $\mathbf{u} \leftrightarrow \mathbf{x} \leftrightarrow \mathbf{y}$ forms a Markov chain. Equation (7) follows from conditioning reduces entropy (Lemma 8).

4) In the range where $L_{\mathrm{CP}}^{*}<L \leqslant L_{\text {weak }}^{*}$, we provide an achievability result whereas there was none in [SG12]. In particular, our result indicates that the capacity is positive in this regime which was open before this work.

5) When $L>L_{\text {weak }}^{*}$, by Equation (8), it seems that our lower bound is no better than that by Sarwate and Gastpar [SG12]. However, in this regime, these two bounds in fact coincide. To see this, note that, by nonweak- $L$-symmetrizability, there is an input distribution $P_{\mathbf{x}} \in \lambda_{\mathbf{x}}$ such that $P_{\mathbf{x}}^{\otimes L}$ is not $L$-symmetrizable. Therefore we could simply take $\mathcal{U}=\{0\}$ and take $\mathbf{u}=0$ to be a constant time-sharing variable. Consequently, $P_{\mathbf{u}, \mathbf{x}_{1}, \cdots, \mathbf{x}_{L}}=P_{\mathbf{x}}^{\otimes L}$ and $I(\mathbf{x} ; \mathbf{y} \mid \mathbf{u})=I(\mathbf{x} ; \mathbf{y})$.

For the same reason, our (conditional) upper bound $C_{L}$ does not contradict the lower bound $\widehat{C}_{L}$ in [SG12].

In summary, the effect of complete positivity is two-fold.

1) The introduction of the notion of CP-symmetrizability moves the critical $L^{*}$ (which we show equals $L_{\mathrm{CP}}^{*}$ ) above which the $L$-list-decoding capacity is positive and at most which the $L$-list-decoding capacity is zero.

2) The complete positivity of the spoofing list reduces the value of the list-decoding capacity expression in the manner of conditioning on a time-sharing variable.

\section{CP-SYMMETRIZATION: AN IMPROVED JAMMING STRATEGY}

We want to show that given $L$, if every $P_{\mathbf{x}} \in \lambda_{\mathbf{x}}$ is $L$-obliviously symmetrizable ${ }^{11}$, then no positive rate can be achieved. That is, any positive rate code $\mathcal{C} \subseteq \mathcal{X}^{n}$ cannot be $L$-list-decodable for $\mathcal{A}_{\text {obli }}$. To this end, we will equip

\footnotetext{
${ }^{11}$ This is equivalent to the condition $L \leqslant L_{\mathrm{CP}}^{*}$.
} 


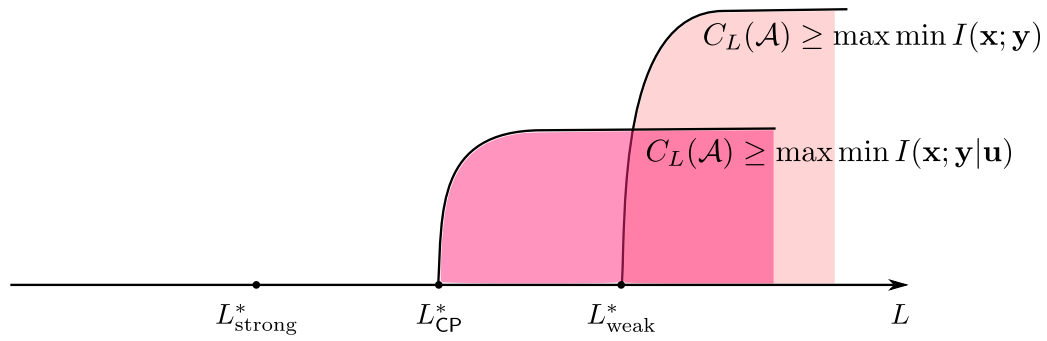

(a) Comparison of achievability results.

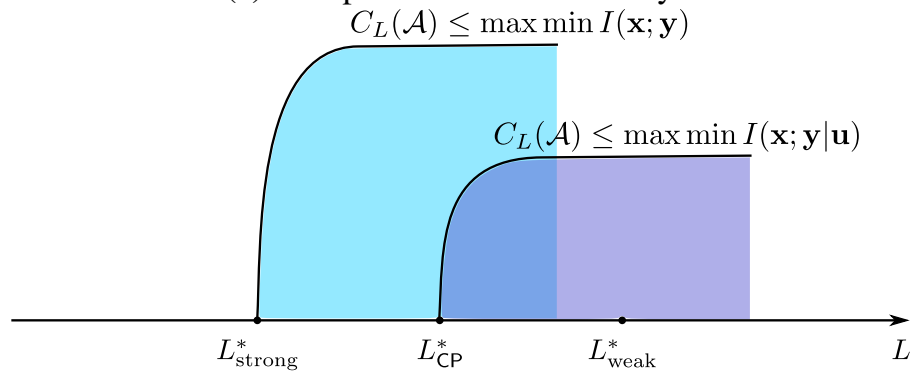

(b) Comparison of capacity upper bounds.

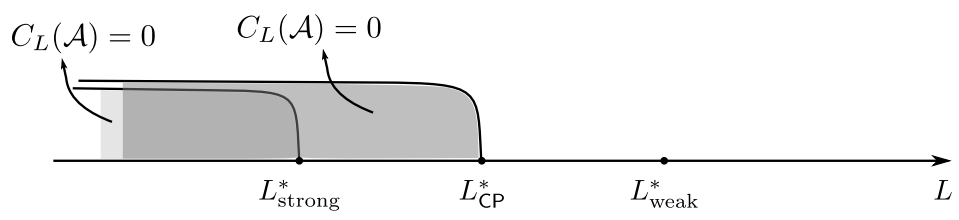

(c) Comparison of symmetrization results.

Fig. 5: Comparisons of achievability, symmetrization converse and capacity upper bounds in this work and [SG12].

James with a jamming strategy and argue that under such a strategy the average probability of Bob's decoding error is at least some constant (independent of the blocklength $n$ ). To describe the jamming strategy, we need the following definitions, observations and lemmas.

Suppose that Alice uses a (deterministic) codebook $\mathcal{C}$. Let $M:=|\mathcal{C}|$. Take a $\lambda$-net $\mathcal{N}_{1}$ of $\Delta(\mathcal{X})$ of size $N_{1}=$ $N_{1}(\lambda,|\mathcal{X}|)$. Take the largest subcode $\mathcal{C}^{\prime} \subset \mathcal{C}$ which is $\left(\lambda, \widehat{P}_{\mathbf{x}}\right)$-constant-composition for some $\widehat{P}_{\mathbf{x}} \in \mathcal{N}_{1}$. Let $M^{\prime}:=\left|\mathcal{C}^{\prime}\right|$. Note that $M^{\prime} \geqslant M / N_{1}$.

Assume $\mathcal{C}=\left\{\underline{x}_{i}\right\}_{i=1}^{M}$ and $\mathcal{C}^{\prime}=\left\{\underline{x}_{j}\right\}_{j=1}^{M^{\prime}}$. Define

$$
\begin{aligned}
\Gamma_{L} & =\Gamma^{\otimes L}(\mathcal{C}):=\left\{\tau_{\underline{x}_{i_{1}}, \cdots, \underline{x}_{i}}: 1 \leqslant i_{1}<\cdots<i_{L} \leqslant M\right\}, \\
\Gamma_{L}^{\prime} & =\Gamma^{\otimes L}\left(\mathcal{C}^{\prime}\right):=\left\{\tau_{\underline{x}_{j_{1}}, \cdots, \underline{x}_{j_{L}}}: 1 \leqslant j_{1}<\cdots<j_{L} \leqslant M^{\prime}\right\} .
\end{aligned}
$$

Theorem 16 (Robust generalized Plotkin, [ZBJ20]). Let $\widehat{P}_{\mathbf{x}} \in \Delta(\mathcal{X})$ and let $\lambda>0$ be a constant. Any $\left(\lambda, \widehat{P}_{\mathbf{x}}\right)$ constant-composition code $\mathcal{C} \subseteq \mathcal{X}^{n}$ satisfying

$$
d\left(\Gamma^{\otimes L}(\mathcal{C}), \mathrm{CP}^{\otimes L}\left(\widehat{P}_{\mathbf{x}}\right)\right)>\varepsilon,
$$

for some constant $\varepsilon \gg \lambda$, has size at most $K=K(\lambda, \varepsilon, L,|\mathcal{X}|)$ (independent of $n$ ).

The above theorem which allows the code to be approximately constant-composition is in fact a slight extension to [ZBJ20]. The proof is similar and we only highlight the differences in Appendix A. 
Theorem 17 (Hypergraph Turán). If an L-uniform hypergraph $\mathcal{H}^{(L)}$ on $M$ vertices contains as subgraphs no complete L-uniform hypergraphs $\mathcal{K}_{K}^{(L)}$ on $K$ vertices, then the edge density of $\mathcal{H}^{(L)}$ is at most

$$
\frac{e\left(\mathcal{H}^{(L)}\right)}{\left(\begin{array}{c}
M \\
L
\end{array}\right)} \leqslant 1-\frac{1}{\left(\begin{array}{l}
K \\
L
\end{array}\right)},
$$

where $e\left(\mathcal{H}^{(L)}\right)$ denotes the number of hyperedges in $\mathcal{H}^{(L)}$.

Remark 11. In [DC83], the author proved a stronger bound

$$
\frac{e\left(\mathcal{H}^{(L)}\right)}{\left(\begin{array}{c}
M \\
L
\end{array}\right)} \leqslant 1-\frac{1}{\left(\begin{array}{c}
K-1 \\
L-1
\end{array}\right)} .
$$

However, the simple bound in Theorem 17 suffices for the purposes of this paper. The readers are encouraged to refer to the survey by Keevash [Kee11] on hypergraph Turán problem.

Lemma 18 (CP-list extraction). Let $\widehat{P}_{\mathbf{x}} \in \Delta(\mathcal{X})$. Fix constants $\lambda>0$ and $\varepsilon \gg \lambda$. For any $\left(\lambda, \widehat{P}_{\mathbf{x}}\right)$-constantcomposition code $\mathcal{C}=\left\{\underline{x}_{i}\right\}_{i=1}^{M} \subseteq \mathcal{X}^{n}$, there exists a constant $\nu=\nu(\lambda, \varepsilon, L,|\mathcal{X}|)>0$ (independent of $n$ ) such that

$$
\frac{1}{\left(\begin{array}{c}
M \\
L
\end{array}\right)}\left|\left\{\tau_{\underline{x}_{i_{1}}, \cdots, \underline{x}_{i_{L}}}: 1 \leqslant i_{1}<\cdots<i_{L} \leqslant M, d\left(\tau_{\underline{x}_{i_{1}}, \cdots, \underline{x}_{i_{L}}}, \mathrm{CP}^{\otimes L}\left(\widehat{P}_{\mathbf{x}}\right)\right) \leqslant \varepsilon\right\}\right| \geqslant \nu .
$$

Proof. Let $\mathcal{H}_{\mathcal{C}}$ be a hypergraph consisting of all codewords in $\mathcal{C}$ as vertices. An $L$-tuple of codewords $\underline{x}_{i_{1}}, \cdots, \underline{x}_{i_{L}}$ $\left(1 \leqslant i_{1}<\cdots<i_{L} \leqslant M\right)$ is connected by a hyperedge if $d\left(\tau_{\underline{x}_{i_{1}}, \cdots, \underline{x}_{i_{L}}}, \mathrm{CP} \otimes L\left(\widehat{P}_{\mathbf{x}}\right)\right)>\varepsilon$. Then by Plotkin bound, no subcode $\mathcal{C}^{\prime}=\left\{\underline{x}_{j}\right\}_{j=1}^{M^{\prime}} \subset \mathcal{C}$ of size larger than $M^{\prime}>K=K(\lambda, \varepsilon, L,|\mathcal{X}|)$ satisfies $d\left(\tau_{x_{j_{1}}, \cdots, \underline{x}_{j_{L}}}, \mathrm{CP}^{\otimes L}\left(\widehat{P}_{\mathbf{x}}\right)\right)>\varepsilon$ for all $1 \leqslant j_{1}<\cdots<j_{L} \leqslant M^{\prime}$. Said differently, $\mathcal{H}_{\mathcal{C}}$ has no clique of size larger than $K$. Then by the hypergraph Turán's theorem (Theorem 17), the edge density of $\mathcal{H}_{\mathcal{C}}$ is at most $1-\nu<1$, where $\nu=\left(\begin{array}{l}K \\ L\end{array}\right)^{-1}$. That is, at least a $\nu$ fraction of order- $L$ joint types of $\mathcal{C}$ are $\varepsilon$-close to $\mathrm{CP}^{\otimes L}\left(\widehat{P}_{\mathbf{x}}\right)$. (See Figure 6.)

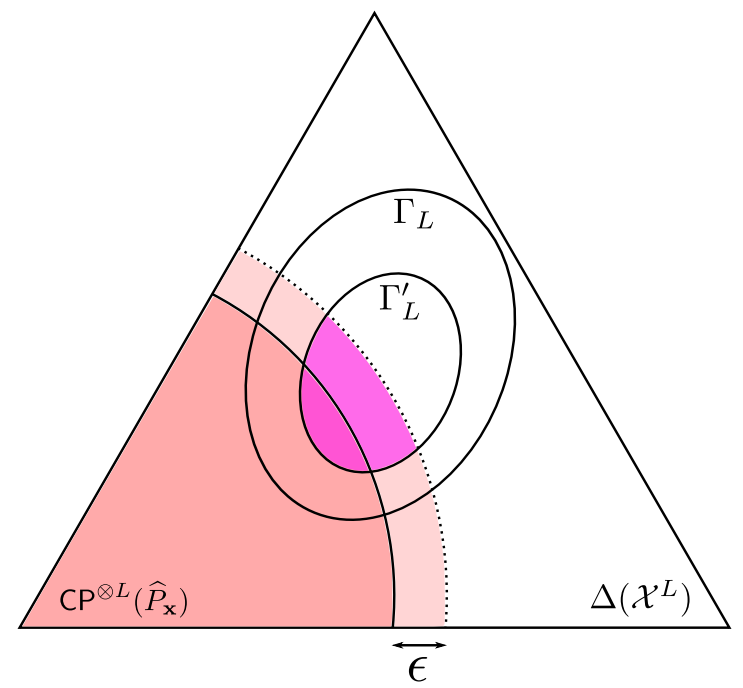

Fig. 6: Any sufficiently large code must contain many completely positive joint types.

For a subset $\mathcal{A} \subseteq \Delta$ of a metric space $\left(\Delta\right.$, dist), let $(\mathcal{A})_{\varepsilon}$ denote its $\varepsilon$-enlargement, i.e., $(\mathcal{A})_{\varepsilon}:=\{a \in \Delta: \operatorname{dist}(a, \mathcal{A}) \leqslant \varepsilon\}$. In particular, a ball centered around a point $a$ of radius $\varepsilon$ can be denoted by $(a)_{\varepsilon}$. 
Take an $\eta$-net $\mathcal{N}_{2}$ of $\left(\mathrm{CP}^{\otimes L}\left(\widehat{P}_{\mathbf{x}}\right)\right)_{\varepsilon} \cap \Delta\left(\mathcal{X}^{L}\right)$ of size $N_{2}:=N_{2}(\varepsilon, \eta, L,|\mathcal{X}|)$. By Lemma 18, we know that the fraction of order- $L$ joint types of $\mathcal{C}^{\prime}$ that are $\varepsilon$-close to $\mathrm{CP}^{\otimes L}\left(\widehat{P}_{\mathbf{x}}\right)$ is at least $\nu$. By Markov's inequality (Lemma 2), there exists a distribution $\widehat{P}_{\mathbf{x}_{1}, \cdots, \mathbf{x}_{L}} \in \mathcal{N}_{2}$ such that

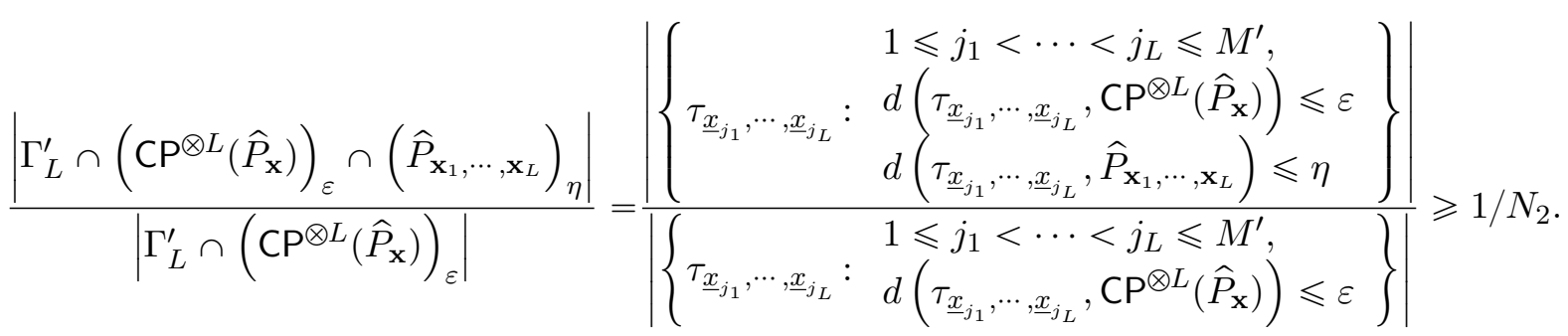

(See. Figure 7.) That is

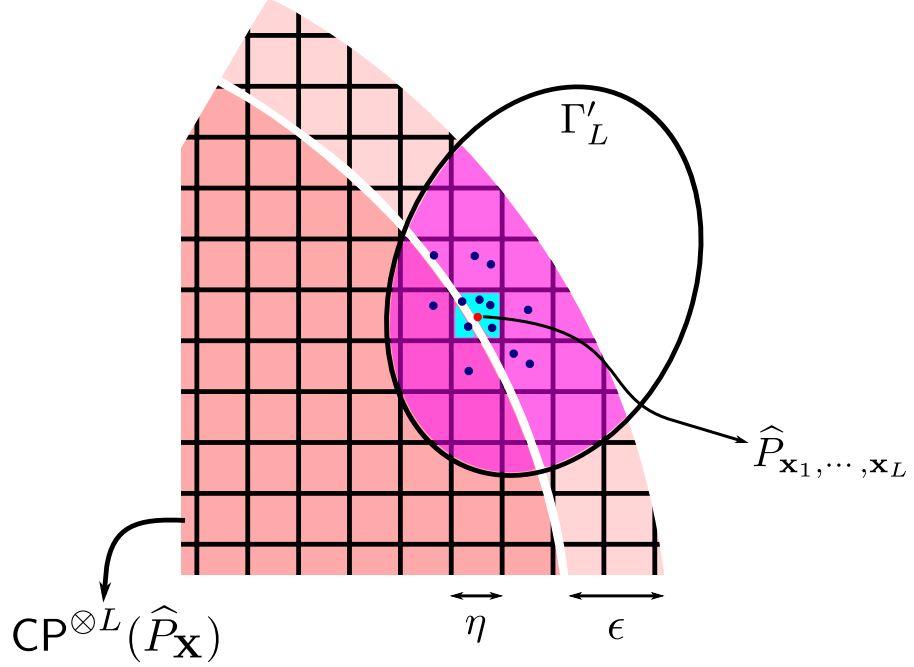

Fig. 7: Quantize the set of joint types and find an approximately equicoupled subcode.

$$
\left|\Gamma_{L}^{\prime} \cap\left(\mathrm{CP}^{\otimes L}\left(\widehat{P}_{\mathbf{x}}\right)\right)_{\varepsilon} \cap\left(\widehat{P}_{\mathbf{x}_{1}, \cdots, \mathbf{x}_{L}}\right)_{\eta}\right| \geqslant\left|\Gamma_{L}^{\prime} \cap\left(\mathrm{CP}^{\otimes L}\left(\widehat{P}_{\mathbf{x}}\right)\right)_{\varepsilon}\right| / N_{2} \geqslant \frac{\left|\Gamma_{L}^{\prime}\right| \nu}{N_{2}} .
$$

Note that

$$
\left|\Gamma_{L}^{\prime}\right|=\left(\begin{array}{c}
M^{\prime} \\
L
\end{array}\right) \geqslant\left(\begin{array}{c}
M / N_{1} \\
L
\end{array}\right), \quad\left|\Gamma_{L}\right|=\left(\begin{array}{c}
M \\
L
\end{array}\right)
$$

Therefore

$$
\frac{\left|\Gamma_{L}^{\prime} \cap\left(\mathrm{CP}^{\otimes L}\left(\widehat{P}_{\mathbf{x}}\right)\right)_{\varepsilon} \cap\left(\widehat{P}_{\mathbf{x}_{1}, \cdots, \mathbf{x}_{L}}\right)_{\eta}\right|}{\left|\Gamma_{L}\right|} \geqslant \frac{\left(\begin{array}{c}
M / N_{1} \\
L
\end{array}\right) \nu}{\left(\begin{array}{c}
M \\
L
\end{array}\right) N_{2}} \geqslant \frac{\nu\left(\frac{M / N_{1}}{L}\right)^{L}}{N_{2}\left(\frac{M e}{L}\right)^{L}}=\frac{\nu}{N_{2}\left(N_{1} e\right)^{L}}=: c
$$

which is at least a constant, denoted by $c>0$,

Note that $\widehat{P}_{\mathbf{x}_{1}, \cdots, \mathbf{x}_{L}} \in \mathcal{N}_{2} \subset\left(\mathrm{CP}^{\otimes L}\left(\widehat{P}_{\mathbf{x}}\right)\right) \cap \Delta\left(\mathcal{X}^{L}\right)$ may not be exactly $\mathrm{CP}$. We project it back to $\mathrm{CP}^{\otimes L}\left(\widehat{P}_{\mathbf{x}}\right)$ and get $\widetilde{P}_{\mathbf{x}_{1}, \cdots, \mathbf{x}_{L}} \in \mathrm{CP} \otimes L\left(\widehat{P}_{\mathbf{x}}\right)$. That is

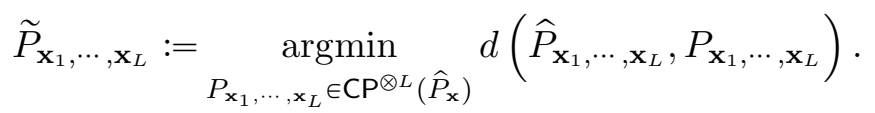

We automatically have

$$
d\left(\widehat{P}_{\mathbf{x}_{1}, \cdots, \mathbf{x}_{L}}, \widetilde{P}_{\mathbf{x}_{1}, \cdots, \mathbf{x}_{L}}\right) \leqslant \varepsilon
$$


We are now ready to specify the jamming strategy James is going to use. Given a (deterministic) codebook $\mathcal{C}$ (which is known to every party), assume $\underline{\mathrm{x}}$ (which James does not know) was transmitted by Alice. James first samples randomly $L$ codewords $\underline{\mathbf{x}}_{1}, \cdots, \underline{\mathbf{x}}_{L}$ from $\mathcal{C}$.

Since $L \leqslant L_{\mathrm{CP}}^{*}$, every $P_{\mathbf{x}} \in \lambda_{\mathbf{x}}$ is $L$-symmetrizable. In particular, $\widehat{P}_{\mathbf{x}} \in \lambda_{\mathbf{x}}$ is also symmetrizable. Since $\widetilde{P}_{\mathbf{x}_{1}, \cdots, \mathbf{x}_{L}}$ constructed above is a completely positive $\widehat{P}_{\mathbf{x}}$-self-coupling, for any CP-decomposition $\left(\widetilde{P}_{\mathbf{u}}, \widetilde{P}_{\mathbf{x} \mid \mathbf{u}}\right)$ of $\widetilde{P}_{\mathbf{x}_{1}, \cdots, \mathbf{x}_{L}}$, there is a symmetrizing distribution $U_{\mathbf{s} \mid \mathbf{u}, \mathbf{x}_{1}, \cdots, \mathbf{x}_{L}} \in \mathcal{U}_{L}$ associated to $\left(\widetilde{P}_{\mathbf{u}}, \widetilde{P}_{\mathbf{x} \mid \mathbf{u}}\right)$. James then sample $\underline{\mathbf{s}} \sim\left[\widetilde{P}_{\mathbf{u}} \widetilde{P}_{\mathbf{x} \mid \mathbf{u}}^{\otimes L} U_{\mathbf{s} \mid \mathbf{u}, \mathbf{x}_{1}, \cdots, \mathbf{x}_{L}}\right]_{\mathbf{s}}^{\otimes n}$

We then briefly sketch why the above strategy enforces the probability of Bob's decoding error to be bounded away from zero. The formal proof is relegated to Appendix B. Assume that for some positive constants $\delta_{i}>0$ $(1 \leqslant i \leqslant \beta)$,

$$
\operatorname{cost}_{i}\left(\left(\widetilde{P}_{\mathbf{u}}, \widetilde{P}_{\mathbf{x} \mid \mathbf{u}}\right), U_{\mathbf{s} \mid \mathbf{u}, \mathbf{x}_{[L]}}\right)=\sum_{\left(u, x_{[L]}, s\right) \in \mathcal{U} \times \mathcal{X}^{L} \times \mathcal{S}} \widetilde{P}_{\mathbf{u}}(u) \widetilde{P}_{\mathbf{x} \mid \mathbf{u}}^{\otimes L}\left(x_{[L]} \mid u\right) U_{\mathbf{s} \mid \mathbf{u}, \mathbf{x}_{[L]}}\left(s \mid u, x_{[L]}\right) B_{i}(s) \leqslant \Lambda_{i}-\delta_{i} .
$$

By Equation (11), it can be shown that

$$
\begin{aligned}
\mid \sum_{\left(u, x_{[L]}, s\right) \in \mathcal{U} \times \mathcal{X}^{L} \times \mathcal{S}} \tau_{\underline{u}, \underline{x}_{\mathcal{L}}}\left(u, x_{[L]}\right) U_{\mathbf{s} \mid \mathbf{u}, \mathbf{x}_{[L]}}\left(s \mid u, x_{[L]}\right) B_{i}(s) & \\
& -\sum_{\left(u, x_{[L]}, s\right) \in \mathcal{U} \times \mathcal{X}^{L} \times \mathcal{S}} \widetilde{P}_{\mathbf{u}}(u) \widetilde{P}_{\mathbf{x} \mid \mathbf{u}}^{\otimes L}\left(x_{[L]} \mid u\right) U_{\mathbf{s} \mid \mathbf{u}, \mathbf{x}_{[L]}}\left(s \mid u, x_{[L]}\right) B_{i}(s) \mid \leqslant g(\eta, \varepsilon) .
\end{aligned}
$$

Therefore

$$
\operatorname{cost}_{i}\left(\tau_{\underline{u}, \underline{x}_{\mathcal{L}}}, U_{\mathbf{s} \mid \mathbf{u}, \mathbf{x}_{[L]}}\right) \leqslant \operatorname{cost}_{i}\left(\left(\widetilde{P}_{\mathbf{u}}, \widetilde{P}_{\mathbf{x} \mid \mathbf{u}}\right), U_{\mathbf{s} \mid \mathbf{u}, \mathbf{x}_{[L]}}\right)+g(\eta, \varepsilon) \leqslant \Lambda_{i}-\delta_{i}+g(\eta, \varepsilon)<\Lambda_{i},
$$

by choosing proper $\eta$ and $\varepsilon$.

Over the randomness of Alice's choice of $\underline{\mathbf{x}}$ and James's choice of $\underline{\mathbf{x}}_{1}, \cdots, \underline{\mathbf{x}}_{L}$, the joint type $\tau_{\underline{\mathbf{x}}_{1}, \cdots, \underline{\mathbf{x}}_{L}}$ is $(\eta+\varepsilon)$ close to $\widetilde{P}_{\mathbf{x}_{[L]}}$ with probability at least $c$, given $\underline{\mathbf{x}}, \underline{\mathbf{x}}_{1}, \cdots, \underline{\mathbf{x}}_{L}$ are distinct (which is not case with probability only $1-\frac{M-1}{M} \frac{M-2}{M} \cdots \frac{M-L}{M}-$ vanishingly small in $n$ ). Since James uses a symmetrizing distribution $U_{\mathbf{s} \mid \mathbf{u}, \mathbf{x}_{[L]}}$ tailored for $\widetilde{P}_{\mathbf{x}_{[L]}}$, the channel is symmetrized under the aforementioned condition. Then from Bob's perspective, any $L$-subset of $\underline{\mathbf{x}}, \underline{\mathbf{x}}_{1}, \cdots, \underline{\mathbf{x}}_{L}$ is equally likely and he could not distinguish them. Consequently, no matter which list-decoder Bob uses, it makes an error with probability at least $1 / L$ - no better than randomly output an $L$-subset of the candidate $(L+1)$-list. The above intuition combined with the calculation in this section can be formalized using arguments along the lines of [CN88b], [Hug97], [SG12]. See Appendix B for details.

\section{Achievability}

In this section, we prove Theorem 13. The proof is along the lines of [CN88b], [Hug97], [SG12].

Assume that $L>L_{\mathrm{CP}}^{*}$. It suffices to take $L=L_{\mathrm{CP}}^{*}+1$. By the definition of CP-symmetrizability (Definition 14), there exists a feasible input distribution $P_{\mathbf{x}} \in \lambda_{\mathbf{x}}$, a CP $P_{\mathbf{x}^{-}}$-self-coupling $P_{\mathbf{x}_{1}, \cdots, \mathbf{x}_{L}} \in \mathrm{CP}^{\otimes L}\left(P_{\mathbf{x}}\right)$ and a CPdecomposition $\left(P_{\mathbf{u}}, P_{\mathbf{x} \mid \mathbf{u}}\right)$ such that there is no symmetrizing distribution $U_{\mathbf{s} \mid \mathbf{u}, \mathbf{x}_{1}, \cdots, \mathbf{x}_{L}} \in \mathcal{U}_{\mathrm{obli}, L \text {-symm }}$ satisfying power constraint $\left[P_{\mathbf{u}} P_{\mathbf{x} \mid \mathbf{u}}^{\otimes L} U_{\mathbf{s} \mid \mathbf{u}, \mathbf{x}_{1}, \cdots, \mathbf{x}_{L}}\right]_{\mathbf{s}} \in \lambda_{\mathbf{s}}$.

Throughout the proof of achievability, fix any distributions $P_{\mathbf{x}} \in \lambda_{\mathbf{x}}$ and $P_{\mathbf{x}_{1}, \cdots, \mathbf{x}_{L}} \in \mathrm{CP}^{\otimes L}\left(P_{\mathbf{x}}\right)$ satisfying the above conditions. Let $P_{\mathbf{x}_{1}, \cdots, \mathbf{x}_{L}}=\sum_{i=1}^{k} \lambda_{i} P_{\mathbf{x}_{i}}^{\otimes L}$ for some $k \geqslant \mathrm{CP}-\operatorname{rk}\left(P_{\mathbf{x}_{1}, \cdots, \mathbf{x}_{L}}\right)$ be any non- $L$-symmetrizable CPdecomposition of $P_{\mathbf{x}_{1}, \cdots, \mathbf{x}_{L}}$. We introduce a time-sharing variable $\mathbf{u}$ supported on $[k]$ with probability mass vector $P_{\mathbf{u}}=\left[\lambda_{1}, \cdots, \lambda_{k}\right]$. By the construction of $\mathbf{u}, P_{\mathbf{u}}$ has no zero atoms. The CP-decomposition can be alternatively written as $P_{\mathbf{x}_{1}, \cdots, \mathbf{x}_{L}}=\left[P_{\mathbf{u}} P_{\mathbf{x} \mid \mathbf{u}}^{\otimes L}\right]_{\mathbf{x}_{1}, \cdots, \mathbf{x}_{L}}$ where $P_{\mathbf{x} \mid \mathbf{u}=i}=P_{\mathbf{x}_{i}}$ for any $i \in[k]$.

Let $\mathcal{U}:=[k]$. For a list of messages $\mathcal{L}=\left\{i_{1}, \cdots, i_{L}\right\}, 1 \leqslant i_{1}<\cdots<i_{L}$, we sometimes use $\underline{v}_{\mathcal{L}}$ to denote the sequence of vectors $\underline{v}_{i_{1}}, \cdots, \underline{v}_{i_{L}}$. Similar notation is used for random variables $\mathbf{v}_{\mathcal{L}}$. 


\section{A. Codebook design}

Lemma 19 (Codeword selection). Fix any $L \in \mathbb{Z}_{>0}, \varepsilon>0$, sufficiently large $n \in \mathbb{Z}_{>0}, M \geqslant L 2^{n \varepsilon}, \underline{u} \in \mathcal{U}^{n}$ of type $P_{\mathbf{u}} \in \Delta(\mathcal{U})$ and conditional type $P_{\mathbf{x} \mid \mathbf{u}} \in \Delta(\mathcal{X} \mid \mathcal{U})$. Let $R=\frac{1}{n} \log \frac{M}{L}$. Then there exist codewords $\underline{x}_{1}, \cdots, \underline{x}_{M} \in \mathcal{X}^{n}$, each of joint type $P_{\mathbf{u}, \mathbf{x}}=P_{\mathbf{u}} P_{\mathbf{x} \mid \mathbf{u}}$ with $\underline{u}$, satisfying that for every $\underline{x} \in \mathcal{X}^{n}, \underline{s} \in \mathcal{S}^{n}$, joint type $P_{\mathbf{u}, \mathbf{x}, \mathbf{x}_{1}, \cdots, \mathbf{x}_{L}, \mathbf{s}} \in$ $\Delta\left(\mathcal{U} \times \mathcal{X} \times \mathcal{X}^{L} \times \mathcal{S}\right)$ and $k \in[L]$

1) if $I(\mathbf{x} ; \mathbf{s} \mid \mathbf{u}) \geqslant \varepsilon$, then

$$
\frac{1}{M}\left|\left\{i \in[M]: \tau_{\underline{u}, \underline{x}_{i}, \underline{s}}=P_{\mathbf{u}, \mathbf{x}, \mathbf{s}}\right\}\right| \leqslant 2^{-n \varepsilon / 2} ;
$$

2) if $I\left(\mathbf{x} ; \mathbf{x}_{k}, \mathbf{s} \mid \mathbf{u}\right) \geqslant\left[R-I\left(\mathbf{x}_{k} ; \mathbf{s} \mid \mathbf{u}\right)\right]^{+}+\varepsilon$, then

$$
\frac{1}{M}\left|\left\{i \in[M]: \exists j \in[M] \backslash\{i\}, \tau_{\underline{u}, \underline{x}_{i}, \underline{x}_{j}, \underline{s}}=P_{\mathbf{u}, \mathbf{x}, \mathbf{x}_{k}, \mathbf{s}}\right\}\right| \leqslant 2^{-n \varepsilon / 2}
$$

3) and

$$
\left|\left\{j \in[M]: \tau_{\underline{u}, \underline{x}, \underline{x}_{j}, \underline{s}}=P_{\mathbf{u}, \mathbf{x}, \mathbf{x}_{k}, \mathbf{s}}\right\}\right| \leqslant 2^{n\left(\left[R-I\left(\mathbf{x}_{k} ; \mathbf{x}, \mathbf{s} \mid \mathbf{u}\right)\right]^{+}+\varepsilon\right)} .
$$

Moreover, if $R<I\left(\mathbf{x}_{k} ; \mathbf{s} \mid \mathbf{u}\right)$ for all $k \in[L]$, then $\underline{x}_{1}, \cdots, \underline{x}_{M}$ can be selected to further satisfy that

$\left.2^{\prime}\right)$ if $I\left(\mathbf{x} ; \mathbf{x}_{[L]}, \mathbf{s} \mid \mathbf{u}\right) \geqslant \varepsilon$, then

$$
\frac{1}{M}\left|\left\{i \in[M]: \exists \mathcal{L} \in\left(\begin{array}{c}
{[M] \backslash\{i\}} \\
L
\end{array}\right), \tau_{\underline{u}, \underline{x}_{i}, \underline{x}_{\mathcal{L}}, \underline{s}}=P_{\mathbf{u}, \mathbf{x}, \mathbf{x}_{[L]}, \mathbf{s}}\right\}\right| \leqslant 2^{-n \varepsilon / 2}
$$

3') and

$$
\left|\left\{\mathcal{L} \in\left(\begin{array}{c}
{[M]} \\
L
\end{array}\right): \tau_{\underline{u}, \underline{x}, \underline{x}_{\mathcal{L}}, \underline{s}}=P_{\mathbf{u}, \mathbf{x}, \mathbf{x}_{[L]}, \mathbf{s}}\right\}\right| \leqslant 2^{n \varepsilon}
$$

\section{B. Decoding rules}

Define the set of joint distributions that $\eta$-approximately (w.r.t. KL-divergence) obey the channel structure:

$$
\mathcal{P}_{\eta}:=\left\{P_{\mathbf{u}, \mathbf{x}, \mathbf{s}, \mathbf{y}} \in \Delta(\mathcal{U} \times \mathcal{X} \times \mathcal{S} \times \mathcal{Y}): D\left(P_{\mathbf{u}, \mathbf{x}, \mathbf{s}, \mathbf{y}} \| P_{\mathbf{u}} P_{\mathbf{x} \mid \mathbf{u}} P_{\mathbf{s}} W_{\mathbf{y} \mid \mathbf{x}, \mathbf{s}}\right) \leqslant \eta, P_{\mathbf{s}} \in \lambda_{\mathbf{s}}\right\} .
$$

Given the codebook $\mathcal{C}=\left\{\underline{x}_{i}\right\}_{i \in[M]}$, the time-sharing sequence $\underline{u} \in \mathcal{U}^{n}$ and Bob's observation $\underline{y} \in \mathcal{Y}^{n}$, the decoder $\psi$ outputs all messages $i \in[M]$ satisfying: there is a jamming vector $\underline{s} \in \Lambda_{\mathbf{S}}$ such that

1) $\tau_{\underline{u}, \underline{x}_{i}, \underline{s}, y} \in \mathcal{P}_{\eta}$;

2) for all $\mathcal{L}^{\prime}=\left\{\underline{x}_{i_{1}}, \cdots, \underline{x}_{i_{L}}\right\}, 1 \leqslant i_{1}<\cdots<i_{L} \leqslant M$ satisfying that

- there is a collection of jamming vectors $\left\{\underline{s}_{j}\right\}_{j \in[L]} \subset \Lambda_{\mathbf{S}}$ such that $\tau_{\underline{u}, \underline{x}_{i}, \underline{s}_{j}, \underline{y}} \in \mathcal{P}_{\eta}$ for each $j \in[L]$,

it holds that $I\left(\mathbf{x}, \mathbf{y} ; \mathbf{x}_{1}, \ldots, \mathbf{x}_{L} \mid \mathbf{u}, \mathbf{s}\right) \leqslant \eta$ where the mutual information is evaluated w.r.t $P_{\mathbf{u}, \mathbf{x}, \mathbf{x}_{1}, \cdots, \mathbf{x}_{L}, \mathbf{s}, \mathbf{y}}=$

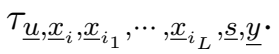

The first step of the decoding rules can be viewed as a list-decoding step. Bob can use the typicality condition in Item 1 to list-decode to a list $\mathcal{L}_{0}$ of at most $\mathcal{O}(\operatorname{poly}(n))$ messages. The second step can be viewed as a tournament. Bob examines all pairs $\left(\mathcal{L}_{1}, \mathcal{L}_{2}\right) \subseteq \mathcal{L}_{0} \times \mathcal{L}_{0}$ of distinct $L$-lists of messages in the big list $\mathcal{L}_{0}$ and determines if $\mathcal{L}_{1}$ or $\mathcal{L}_{2}$ wins. If there is a unique champion in this competition, then the decoder outputs that list.

It is clear that the correct message $i$ will be output with high probability. We also need to argue that such a decoder is a valid list-decoder such that $|\psi(\underline{y})| \leqslant L$ for any channel output $\underline{y} \in \mathcal{Y}^{n}$.

\section{Unambiguity of decoding rules}

Lemma 20. There is no joint distribution $P_{\mathbf{u}, \mathbf{x}_{[L+1]}, \mathbf{s}_{[L+1]}, \mathbf{y}}$ that simultaneously satisfies

$$
P_{\mathbf{u}, \mathbf{x}_{i}}=P_{\mathbf{u}, \mathbf{x}}, P_{\mathbf{u}, \mathbf{x}_{i}, \mathbf{s}_{i}, \mathbf{y}} \in \mathcal{P}_{\eta}, I\left(\mathbf{x}_{i}, \mathbf{y} ; \mathbf{x}_{[L+1] \backslash\{i\}} \mid \mathbf{u}, \mathbf{s}_{i}\right) \leqslant \eta,
$$

for all $i \in[L+1]$. 


\section{Analysis of average error probability}

Fix a non- $L$-symmetrizable $P_{\mathbf{x}}$ and a corresponding $P_{\mathbf{x}_{1}, \cdots, \mathbf{x}_{L}} \in \mathrm{CP}^{\otimes L}\left(P_{\mathbf{x}}\right)$ which induces $P_{\mathbf{u}}$ and $P_{\mathbf{x} \mid \mathbf{u}}$. For each $j \in[\beta]$, define the minimum power that James has to spend to symmetrize the channel,

$$
\Lambda_{j}^{*}\left(P_{\mathbf{u}}, P_{\mathbf{x} \mid \mathbf{u}}\right):=\min _{U_{\mathbf{s} \mid \mathbf{u}, \mathbf{x}[L]} \in \mathcal{U}_{\mathrm{obli}, L-\mathrm{symm}}} \sum_{\left(u, x_{[L]}, s\right) \in \mathcal{U} \times \mathcal{X}^{L} \times \mathcal{S}} P_{\mathbf{u}}(u) P_{\mathbf{x} \mid \mathbf{u}}^{\otimes L}\left(x_{[L]} \mid u\right) U_{\mathbf{s} \mid \mathbf{u}, \mathbf{x}_{[L]}}\left(s \mid u, x_{[L]}\right) B_{j}(s) .
$$

By non- $L$-symmetrizability, we know that there must exist a $j \in[\beta]$ such that $\Lambda_{j}^{*}\left(P_{\mathbf{u}}, P_{\mathbf{x} \mid \mathbf{u}}\right)>\Lambda_{j}$. Focus on that $j$ from now on. Fix an arbitrary $\underline{s} \in \Lambda_{\mathbf{s}}$.

Define the set of bad messages

$$
\mathcal{M}(\underline{u}):=\{m \in[M]: I(\mathbf{x} ; \mathbf{s} \mid \mathbf{u})>\varepsilon\},
$$

where the mutual information is evaluated w.r.t. the joint distribution $P_{\mathbf{u}, \mathbf{x}, \mathbf{s}}=\tau_{\underline{u}, \underline{x}_{m}, \underline{s}}$.

According to the definition of the list-decoder (Section X-B), for any fixed $\underline{s} \in \Lambda_{\mathbf{S}}$, a list-decoding error occurs only if: codeword $\underline{x}_{i}$ corresponding to message $i$ was sent by Alice and $y$ was received by Bob, but

1) either $\left(\underline{u}, \underline{x}_{i}, \underline{s}, \underline{y}\right)$ are not jointly typical in the sense that $\tau_{\underline{u}, \underline{x}_{i}, \underline{s}, \underline{y}} \notin \mathcal{P}_{\eta}$;

2) or a spoofing list of size $L$ that does not contain message $i$ confuses Bob, i.e., there is an $L$-list $\mathcal{L}^{\prime} \in\left({ }^{[M] \backslash\{i\}}\right)$ such that the joint type $P_{\mathbf{u}, \mathbf{x}_{i}, \mathbf{x}_{\mathcal{L}^{\prime}}, \mathbf{s}, \mathbf{y}} \in \Delta^{(n)}\left(\mathcal{U} \times \mathcal{X}^{L+1} \times \mathcal{S} \times \mathcal{Y}\right)$ of the tuple $\left(\underline{u}, \underline{x}_{i}, \underline{x}_{\mathcal{L}^{\prime}}, \underline{s}, y\right)$ satisfies the following conditions:

a) $\left(\underline{u}, \underline{x}_{i}, \underline{s}, \underline{y}\right)$ are jointly typical: $P_{\mathbf{u}, \mathbf{x}_{i}, \mathbf{s}, \mathbf{y}} \in \mathcal{P}_{\eta}$;

b) each codeword corresponding to a candidate message $i^{\prime}$ in the competing list $\mathcal{L}^{\prime}$ is also typical: for each $i^{\prime} \in \mathcal{L}^{\prime}$, there exists an $\underline{s}_{i^{\prime}} \in \Lambda_{\mathbf{S}}$ such that $\left(\underline{u}, \underline{x}_{i^{\prime}}, \underline{s}_{i^{\prime}}, \underline{y}\right)$ has type $P_{\mathbf{u}, \mathbf{x}_{i^{\prime}}, \mathbf{s}_{i^{\prime}}, \mathbf{y}} \in \mathcal{P}_{\eta}$;

c) and finally $I\left(\mathbf{x}_{i}, \mathbf{y} ; \mathbf{x}_{\mathcal{L}^{\prime}} \mid \mathbf{u}, \mathbf{s}\right)>\eta$.

Motivated by the above conditions, we define the set of bad distributions

$$
\mathcal{D}:=\left\{\begin{aligned}
& P_{\mathbf{u}, \mathbf{x}, \mathbf{s}, \mathbf{y}} \in \mathcal{P}_{\eta} \\
& P_{\mathbf{u}, \mathbf{x}, \mathbf{x}_{[L]}, \mathbf{s}, \mathbf{y}} \in \Delta\left(\mathcal{U} \times \mathcal{X} \times \mathcal{X}^{L} \times \mathcal{S} \times \mathcal{Y}\right): \quad \forall i \in \mathcal{L}, \exists \mathbf{s}_{i}, P_{\mathbf{u}, \mathbf{x}_{i}, \mathbf{s}_{i}, \mathbf{y}} \in \mathcal{P}_{\eta} \\
& I\left(\mathbf{x}, \mathbf{y} ; \mathbf{x}_{[L]} \mid \mathbf{u}, \mathbf{s}\right)> \eta
\end{aligned}\right\}
$$

The average error probability under $\underline{s}$ is

$$
\begin{aligned}
P_{\mathrm{e}, \mathrm{avg}}(\underline{s})= & \frac{1}{M} \sum_{m \in[M]} P_{\mathrm{e}}(m, \underline{s}) \leqslant \frac{1}{M} \sum_{m \in \mathcal{M}(\underline{u})} P_{\mathrm{e}}(m, \underline{s})+\frac{1}{M} \sum_{m \in[M] \backslash \mathcal{M}(\underline{u})} P_{\mathrm{e}}(m, \underline{s}) \\
\leqslant & \frac{1}{M}|\{m \in[M]: I(\mathbf{x} ; \mathbf{s} \mid \mathbf{u})>\varepsilon\}| \\
& +\frac{1}{M} \sum_{m \in[M] \backslash \mathcal{M}(\underline{u})} \sum_{y \in \mathcal{Y}_{n}: \tau_{\underline{u}, \underline{x}_{m}, \underline{s}, \underline{y}} \notin \mathcal{P}_{\eta}} W\left(\underline{y} \mid \underline{x}_{m}, \underline{s}\right) \\
& +\frac{1}{M} \sum_{m \in[M] \backslash \mathcal{M}(\underline{u}) \tau_{\mathbf{u}, \mathbf{x}, \mathbf{x}[L], \mathbf{s}, \mathbf{y}} \in \mathcal{D}} P_{\mathrm{e}}\left(m, \underline{s}, \tau_{\mathbf{u}, \mathbf{x}, \mathbf{x}_{[L]}, \mathbf{s}, \mathbf{y}}\right),
\end{aligned}
$$

where

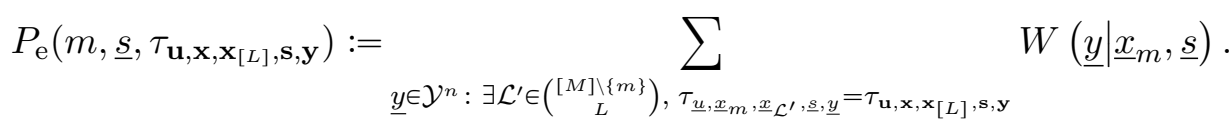

By Equation (13), Term (22) is at most

$$
\frac{1}{M}|\{m \in[M]: I(\mathbf{x} ; \mathbf{s} \mid \mathbf{u})>\varepsilon\}|=\frac{1}{M} \sum_{\substack{\tau_{\mathbf{u}, \mathbf{x}, \mathbf{s}} \in \Delta^{(n)}(\mathcal{U} \times \mathcal{X} \times \mathcal{S}): \\ \tau_{\mathbf{u}, \mathbf{x}}=P_{\mathbf{u}} P_{\mathbf{x} \mid \mathbf{u}, \tau_{\mathbf{s}}=\tau_{\underline{s}}}}}\left|\left\{m \in[M]: \tau_{\underline{u}, \underline{x}_{m}, \underline{s}}=P_{\mathbf{u}, \mathbf{x}, \mathbf{s}}\right\}\right| \mathbb{1}\{I(\mathbf{x} ; \mathbf{s} \mid \mathbf{u})>\varepsilon\} \leqslant 2^{-n \varepsilon / 2} .
$$

By Sanov's theorem (Lemma 4), Term (23) is dot less than

$$
\sup _{P_{\mathbf{u}, \mathbf{x}, \mathbf{s}, \mathbf{y}} \notin \mathcal{P}_{\eta}: I(\mathbf{x} ; \mathbf{s} \mid \mathbf{u})>\varepsilon} 2^{-n D\left(P_{\mathbf{u}, \mathbf{x}, \mathbf{s}, \mathbf{y}} \| P_{\mathbf{u}, \mathbf{x}, \mathbf{s}} W_{\mathbf{y} \mid \mathbf{x}, \mathbf{s}}\right)}=\sup _{P_{\mathbf{u}, \mathbf{x}, \mathbf{s}, \mathbf{y}} \notin \mathcal{P}_{\eta}: I(\mathbf{x} ; \mathbf{s} \mid \mathbf{u})>\varepsilon} 2^{-n\left(D\left(P_{\mathbf{u}, \mathbf{x}, \mathbf{s}, \mathbf{y}} \| P_{\mathbf{u}} P_{\mathbf{x} \mid \mathbf{u}} P_{\mathbf{s}} W_{\mathbf{y} \mid \mathbf{x}, \mathbf{s}}\right)-I(\mathbf{x} ; \mathbf{s} \mid \mathbf{u})\right)} \leqslant 2^{-n(\eta-\varepsilon)} .
$$


The inequality follows from the definition of $\mathcal{P}_{\eta}$ (Equation (18)) and the condition $I(\mathbf{x} ; \mathbf{s} \mid \mathbf{u})>\varepsilon$.

In what follows, we focus on Term (24). We further define the following sets of distributions,

$$
\begin{aligned}
& \mathcal{D}_{1}:=\left\{P_{\mathbf{u}, \mathbf{x}, \mathbf{x}_{[L]}, \mathbf{s}, \mathbf{y}} \in \Delta\left(\mathcal{U} \times \mathcal{X} \times \mathcal{X}^{L} \times \mathcal{S} \times \mathcal{Y}\right): R<\min _{i \in[L]} I\left(\mathbf{x}_{i} ; \mathbf{s} \mid \mathbf{u}\right)\right\}, \\
& \mathcal{D}_{2}:=\left\{P_{\mathbf{u}, \mathbf{x}, \mathbf{x}_{[L]}, \mathbf{s}, \mathbf{y}} \in \Delta\left(\mathcal{U} \times \mathcal{X} \times \mathcal{X}^{L} \times \mathcal{S} \times \mathcal{Y}\right): I\left(\mathbf{x} ; \mathbf{x}_{[L]}, \mathbf{s} \mid \mathbf{u}\right)>\varepsilon\right\} .
\end{aligned}
$$

Now Term (24) can be decomposed as

$$
\begin{aligned}
& \frac{1}{M} \sum_{m \in[M] \backslash \mathcal{M}(\underline{u})} \sum_{\tau_{\mathbf{u}, \mathbf{x}, \mathbf{x}}{ }_{[L]}, \mathbf{s}, \mathbf{y} \in \mathcal{D} \cap \mathcal{D}_{1} \cap \mathcal{D}_{2}} P_{\mathrm{e}}\left(m, \underline{s}, \tau_{\mathbf{u}, \mathbf{x}, \mathbf{x}_{[L]}, \mathbf{s}, \mathbf{y}}\right) \\
& +\frac{1}{M} \sum_{m \in[M] \backslash \mathcal{M}(\underline{u})} \sum_{\tau_{\mathbf{u}, \mathbf{x}, \mathbf{x}[L], \mathbf{s}, \mathbf{y}} \in \mathcal{D} \cap \mathcal{D}_{1} \cap \mathcal{D}_{2}^{c}} P_{\mathrm{e}}\left(m, \underline{s}, \tau_{\mathbf{u}, \mathbf{x}, \mathbf{x}_{[L]}, \mathbf{s}, \mathbf{y}}\right) \\
& +\frac{1}{M} \sum_{m \in[M] \backslash \mathcal{M}(\underline{u}) \tau_{\mathbf{u}, \mathbf{x}, \mathbf{x}[L], \mathbf{s}, \mathbf{y}} \in \mathcal{D} \cap \mathcal{D}_{1}^{c}} P_{\mathrm{e}}\left(m, \underline{s}, \tau_{\mathbf{u}, \mathbf{x}, \mathbf{x}_{[L]}, \mathbf{s}, \mathbf{y}}\right) .
\end{aligned}
$$

Term (27) can be bounded as follows

$$
\begin{aligned}
& \frac{1}{M} \sum_{m \in[M] \backslash \mathcal{M}(\underline{u})} \sum_{\tau_{\mathbf{u}, \mathbf{x}, \mathbf{x}}[L], \mathbf{s}, \mathbf{y} \in \mathcal{D} \cap \mathcal{D}_{1} \cap \mathcal{D}_{2}} \sum_{\underline{y} \in \mathcal{Y}^{n}} W\left(\underline{y} \mid \underline{x}_{m}, \underline{s}\right) \mathbb{1}\left\{\exists \mathcal{L}^{\prime} \in\left(\begin{array}{c}
{[M] \backslash\{m\}} \\
L
\end{array}\right), \tau_{\underline{u}^{\prime}, \underline{x}_{m}, \underline{x}^{\prime}, \underline{s}, \underline{y}}=\tau_{\mathbf{u}, \mathbf{x}, \mathbf{x}_{[L]}, \mathbf{s}, \mathbf{y}}\right\} \\
& \leqslant \frac{1}{M} \sum_{\tau_{\mathbf{u}, \mathbf{x}, \mathbf{x}[L], \mathbf{s}, \mathbf{y}} \in \mathcal{D}_{1} \cap \mathcal{D}_{2}} \sum_{m \in[M]} \sum_{y \in \mathcal{Y}^{n}} W\left(\underline{y} \mid \underline{x}_{m}, \underline{s}\right) \mathbb{1}\left\{\exists \mathcal{L}^{\prime} \in\left(\begin{array}{c}
{[M] \backslash\{m\}} \\
L
\end{array}\right), \tau_{\underline{u}^{\prime}, \underline{x}_{m}, \underline{x}_{\mathcal{L}^{\prime}}, \underline{s}}=\tau_{\mathbf{u}, \mathbf{x}, \mathbf{x}_{[L]}, \mathbf{s}}\right\}
\end{aligned}
$$

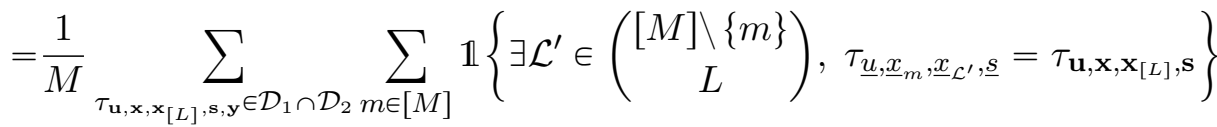

$$
\begin{aligned}
& \leqslant \frac{1}{M} \sum_{\tau_{\mathbf{u}, \mathbf{x}, \mathbf{x}[L], \mathbf{s}, \mathbf{y}} \in \mathcal{D}_{1} \cap \mathcal{D}_{2}}\left|\left\{m \in[M]: \exists \mathcal{L}^{\prime} \in\left(\begin{array}{c}
{[M] \backslash\{m\}} \\
L
\end{array}\right), \tau_{\underline{u}_{,}, \underline{x}_{m}, \underline{x}_{\mathcal{L}^{\prime}}, \underline{s}}=\tau_{\mathbf{u}, \mathbf{x}, \mathbf{x}_{[L]}, \mathbf{s}}\right\}\right| \\
& \leqslant 2^{-n \varepsilon / 2} \text {. }
\end{aligned}
$$

Inequality (30) follows from Equation (16).

Term (28) can be bounded as follows.

$$
\begin{aligned}
& \frac{1}{M} \sum_{m \in[M] \backslash \mathcal{M}(\underline{u})} \sum_{\tau_{\mathbf{u}, \mathbf{x}, \mathbf{x}}[L], \mathbf{s}, \mathbf{y}} \in \mathcal{D} \cap \mathcal{D}_{1} \cap \sum_{\mathcal{D}_{2}^{c}} \sum_{y \in \mathcal{Y}^{n}} W\left(\underline{y} \mid \underline{x}_{m}, \underline{s}\right) \mathbb{1}\left\{\exists \mathcal{L}^{\prime} \in\left(\begin{array}{c}
{[M] \backslash\{m\}} \\
L
\end{array}\right), \tau_{\underline{u}^{\prime}, \underline{x}_{m}, \underline{x}_{\mathcal{L}^{\prime}}, \underline{s}, \underline{y}}=\tau_{\mathbf{u}, \mathbf{x}, \mathbf{x}[L], \mathbf{s}, \mathbf{y}}\right\}
\end{aligned}
$$

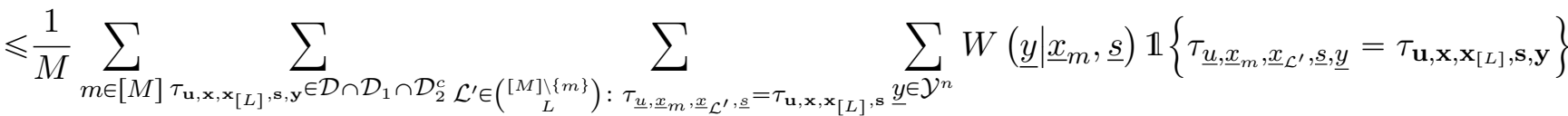

$$
\begin{aligned}
& \leqslant \frac{1}{M} \sum_{m \in[M]} \sum_{\tau_{\mathbf{u}, \mathbf{x}, \mathbf{x}}^{[L]}, \mathbf{s}, \mathbf{y} \in \mathcal{D} \cap \mathcal{D}_{1} \cap \mathcal{D}_{2}^{c}}\left|\left\{\mathcal{L}^{\prime} \in\left(\begin{array}{c}
{[M] \backslash\{m\}} \\
L
\end{array}\right): \tau_{\underline{u}_{x}, \underline{x}_{m}, \underline{x}_{\mathcal{L}^{\prime}}, \underline{s}}=\tau_{\mathbf{u}, \mathbf{x}, \mathbf{x}_{[L]}, \mathbf{s}}\right\}\right| 2^{-n D\left(P_{\mathbf{u}, \mathbf{x}, \mathbf{x}}[L], \mathbf{s}, \mathbf{y} \| P_{\mathbf{u}, \mathbf{x}, \mathbf{x}[L], \mathbf{s}} W_{\mathbf{y} \mid \mathbf{x}, \mathbf{s}}\right)}
\end{aligned}
$$

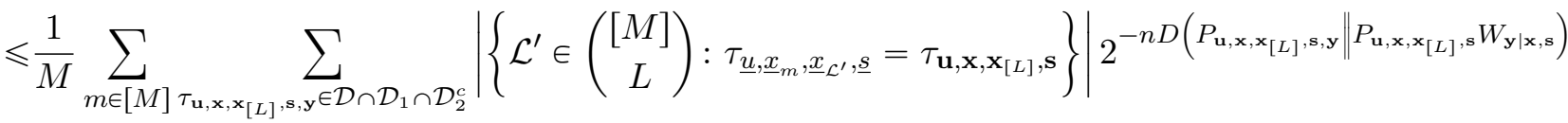

$$
\begin{aligned}
& \leqslant \sum_{\tau_{\mathbf{u}, \mathbf{x}, \mathbf{x}[L]}, \mathbf{s}, \mathbf{y} \in \mathcal{D} \cap \mathcal{D}_{1} \cap \mathcal{D}_{2}^{c}} 2^{n \varepsilon} 2^{-n I\left(\mathbf{y} ; \mathbf{u}, \mathbf{x}_{[L]} \mid \mathbf{x}, \mathbf{s}\right)} \\
& =\sum_{\tau_{\mathbf{u}, \mathbf{x}, \mathbf{x}}[L], \mathbf{s}, \mathbf{y} \in \mathcal{D} \cap \mathcal{D}_{1} \cap \mathcal{D}_{2}^{c}} 2^{n \varepsilon} 2^{-n\left(I(\mathbf{y} ; \mathbf{u} \mid \mathbf{x}, \mathbf{s})+I\left(\mathbf{y} ; \mathbf{x}_{[L]} \mid \mathbf{u}, \mathbf{x}, \mathbf{s}\right)\right)} \\
& \leqslant \sum_{\tau_{\mathbf{u}, \mathbf{x}, \mathbf{x}[L]}, \mathbf{s}, \mathbf{y} \in \mathcal{D} \cap \mathcal{D}_{1} \cap \mathcal{D}_{2}^{c}} 2^{n \varepsilon} 2^{-n\left(I\left(\mathbf{x}, \mathbf{y} ; \mathbf{x}_{[L]} \mid \mathbf{s}, \mathbf{u}\right)-I\left(\mathbf{x} ; \mathbf{x}_{[L]} \mid \mathbf{u}, \mathbf{s}\right)\right)}
\end{aligned}
$$




$$
\begin{aligned}
& \leqslant \sum_{\tau_{\mathbf{u}, \mathbf{x}, \mathbf{x}[L], \mathbf{s}, \mathbf{y}} \in \mathcal{D} \cap \mathcal{D}_{1} \cap \mathcal{D}_{2}^{c}} 2^{n \varepsilon} 2^{-n\left(I\left(\mathbf{x}, \mathbf{y} ; \mathbf{x}_{[L]} \mid \mathbf{s}, \mathbf{u}\right)-\left(I\left(\mathbf{x} ; \mathbf{x}_{[L]}, \mathbf{s} \mid \mathbf{u}\right)-I(\mathbf{x} ; \mathbf{s} \mid \mathbf{u})\right)\right)} \\
& \leqslant \sum_{\tau_{\mathbf{u}, \mathbf{x}, \mathbf{x}[L]}, \mathbf{s}, \mathbf{y} \in \mathcal{D} \cap \mathcal{D}_{1} \cap \mathcal{D}_{2}^{c}} 2^{n \varepsilon} 2^{-n\left(I\left(\mathbf{x}, \mathbf{y} ; \mathbf{x}_{[L]} \mid \mathbf{s}, \mathbf{u}\right)-I\left(\mathbf{x} ; \mathbf{x}_{[L]}, \mathbf{s} \mid \mathbf{u}\right)\right)} \\
& \leqslant 2^{n \varepsilon} 2^{-n(\eta-\varepsilon)}=2^{-n(\eta-2 \varepsilon)} \text {. }
\end{aligned}
$$

Inequality (31) is by Sanov's theorem (Lemma 4). Inequality (32) is by Equation (17) given $\tau_{\mathbf{u}, \mathbf{x}, \mathbf{x}_{[L]}, \mathbf{s}, \mathbf{y}} \in \mathcal{D}_{1}$ (see Equation (25)). Inequality (33) is by the definition of $\mathcal{D}$ (Equation (21)) and $\mathcal{D}_{2}$ (Equation (26)).

We now turn to the last term Term (29). For each $\tau_{\mathbf{u}, \mathbf{x}, \mathbf{x}_{[L]}, \mathbf{s}, \mathbf{y}} \in \mathcal{D}_{1}^{c}$, there must be an $i_{\tau} \in[L]$ such that $R>I\left(\mathbf{x}_{i_{\tau}} ; \mathbf{s} \mid \mathbf{u}\right)$. Define

$$
\mathcal{D}_{3}:=\left\{\tau_{\mathbf{u}, \mathbf{x}, \mathbf{x}_{[L]}, \mathbf{s}, \mathbf{y}} \in \Delta^{(n)}\left(\mathcal{U} \times \mathcal{X} \times \mathcal{X}^{L} \times \mathcal{S} \times \mathcal{Y}\right): I\left(\mathbf{x} ; \mathbf{x}_{i_{\tau}}, \mathbf{s} \mid \mathbf{u}\right)-\left[R-I\left(\mathbf{x}_{i_{\tau}} ; \mathbf{s} \mid \mathbf{u}\right)\right]^{+} \geqslant \varepsilon\right\} .
$$

Term (29) is at most

$$
\begin{aligned}
& \frac{1}{M} \sum_{m \in[M] \backslash \mathcal{M}(\underline{u})} \sum_{\tau_{\mathbf{u}, \mathbf{x}, \mathbf{x}}^{[L]}, \mathbf{s , y} \in \mathcal{D} \cap \mathcal{D}_{1}^{c}} \sum_{y \in \mathcal{Y}^{n}} W\left(\underline{y} \underline{x}_{m}, \underline{s}\right) \mathbb{1}\left\{\exists \mathcal{L}^{\prime} \in\left(\begin{array}{c}
{[M] \backslash\{m\}} \\
L
\end{array}\right), \tau_{\underline{u}, \underline{x}_{m}, \underline{x}_{\mathcal{L}^{\prime}}, \underline{s}, \underline{y}}=\tau_{\mathbf{u}, \mathbf{x}, \mathbf{x}, L], \mathbf{s}, \mathbf{y}}\right\} \\
& \leqslant \frac{1}{M} \sum_{m \in[M] \backslash \mathcal{M}(\underline{u})} \sum_{\tau_{\mathbf{u}, \mathbf{x}, \mathbf{x}}[L], \mathbf{s}, \mathbf{y} \in \mathcal{D} \cap \mathcal{D}_{1}^{c}} \sum_{\underline{y} \in \mathcal{Y}^{n}} W\left(\underline{y} \mid \underline{x}_{m}, \underline{s}\right) \mathbb{1}\left\{\exists m^{\prime} \in[M] \backslash\{m\}, \tau_{\underline{u}^{\prime}, \underline{x}_{m}, \underline{x}_{m^{\prime}}, \underline{s}, \underline{y}}=\tau_{\mathbf{u}, \mathbf{x}, \mathbf{x}_{i_{\tau}}, \mathbf{s}, \mathbf{y}}\right\} \\
& \leqslant \frac{1}{M} \sum_{m \in[M] \backslash \mathcal{M}(\underline{u})} \sum_{\tau_{\mathbf{u}, \mathbf{x}, \mathbf{x}[L]}, \mathbf{s}, \mathbf{y} \in \mathcal{D} \cap \mathcal{D}_{1}^{c} \cap \mathcal{D}_{3}} \sum_{\underline{y} \in \mathcal{Y}^{n}} W\left(\underline{y} \mid \underline{x}_{m}, \underline{s}\right) \mathbb{1}\left\{\exists m^{\prime} \in[M] \backslash\{m\}, \tau_{\underline{u}, \underline{x}_{m}, \underline{x}_{m^{\prime}}, \underline{s}, \underline{y}}=\tau_{\mathbf{u}, \mathbf{x}, \mathbf{x}_{i \tau}, \mathbf{s}, \mathbf{y}}\right\} \\
& +\frac{1}{M} \sum_{m \in[M] \backslash \mathcal{M}(\underline{u})} \sum_{\tau_{\mathbf{u}, \mathbf{x}, \mathbf{x}}[L], \mathbf{s}, \mathbf{y} \in \mathcal{D} \cap \mathcal{D}_{1}^{c} \cap \mathcal{D}_{3}^{c}} \sum_{y \in \mathcal{Y}^{n}} W\left(\underline{y} \mid \underline{x}_{m}, \underline{s}\right) \mathbb{1}\left\{\exists m^{\prime} \in[M] \backslash\{m\}, \tau_{\underline{u}, \underline{x}_{m}, \underline{x}_{m^{\prime}}, \underline{s}, \underline{y}}=\tau_{\left.\mathbf{u}, \mathbf{x}, \mathbf{x}_{i_{\tau}}, \mathbf{s}, \mathbf{y}\right\} .}\right.
\end{aligned}
$$

Term (34) is at most

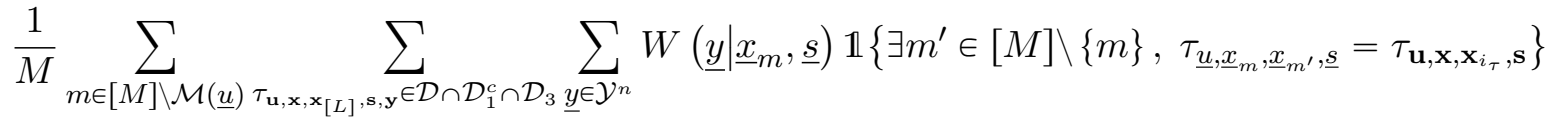

$$
\begin{aligned}
& \leqslant \frac{1}{M} \sum_{\tau_{\mathbf{u}, \mathbf{x}, \mathbf{x}[L]}, \mathbf{s}, \mathbf{y}} \in \mathcal{D} \cap \mathcal{D}_{1}^{c} \cap \mathcal{D}_{3} \sum_{m \in[M]} \mathbb{1}\left\{\exists m^{\prime} \in[M] \backslash\{m\}, \tau_{\underline{u}^{\prime}, \underline{x}_{m}, \underline{x}_{m^{\prime}}, \underline{s}}=\tau_{\mathbf{u}, \mathbf{x}, \mathbf{x}_{i_{\tau}}, \mathbf{s}}\right\} \\
& =\frac{1}{M} \sum_{\tau_{\mathbf{u}, \mathbf{x}, \mathbf{x}[L]}, \mathbf{s}, \mathbf{y} \in \mathcal{D} \cap \mathcal{D}_{1}^{c} \cap \mathcal{D}_{3}}\left|\left\{m \in[M]: \exists m^{\prime} \in[M] \backslash\{m\}, \tau_{\underline{u}, \underline{x}_{m}, \underline{x}_{m^{\prime}}, \underline{s}}=\tau_{\mathbf{u}, \mathbf{x}, \mathbf{x}_{i_{\tau}}, \mathbf{s}}\right\}\right| \\
& \leqslant 2^{-n \varepsilon / 2}
\end{aligned}
$$

where Inequality (36) follows from Equation (14).

Term (35) is at most

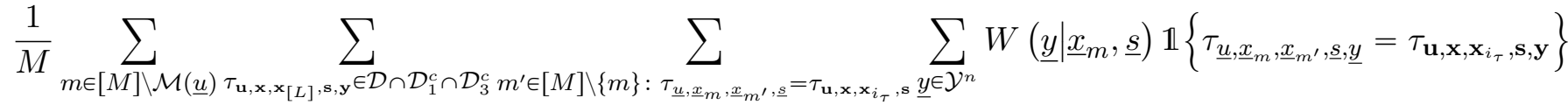

$$
\begin{aligned}
& \leqslant \frac{1}{M} \sum_{m \in[M]} \sum_{\tau_{\mathbf{u}, \mathbf{x}, \mathbf{x}} \sum_{[L]}, \mathbf{s}, \mathbf{y} \in \mathcal{D} \cap \mathcal{D}_{1}^{c} \cap \mathcal{D}_{3}^{c} m^{\prime} \in[M]: \sum_{\tau_{\underline{u},}, \underline{m}_{m}, \underline{x}_{m^{\prime}}, \underline{s}}=\tau_{\mathbf{u}, \mathbf{x}, \mathbf{x}_{i_{\tau}}, \mathbf{s}}} 2^{-n I\left(\mathbf{y} ; \mathbf{x}_{i_{\tau}} \mid \mathbf{u}, \mathbf{x}, \mathbf{s}\right)} \\
& =\frac{1}{M} \sum_{m \in[M]} \sum_{\tau_{\mathbf{u}, \mathbf{x}, \mathbf{x}[L]}, \mathbf{s}, \mathbf{s} \in \mathcal{D} \cap \mathcal{D}_{1}^{c} \cap \mathcal{D}_{3}^{c}}\left|\left\{m^{\prime} \in[M]: \tau_{\underline{u}, \underline{x}_{m}, \underline{x}_{m^{\prime}}, \underline{s}}=\tau_{\mathbf{u}, \mathbf{x}, \mathbf{x}_{i_{\tau}}, \mathbf{s}}\right\}\right| 2^{-n I\left(\mathbf{y} ; \mathbf{x}_{i_{\tau}} \mid \mathbf{u}, \mathbf{x}, \mathbf{s}\right)} \\
& \leqslant \sum_{\tau_{\mathbf{u}, \mathbf{x}, \mathbf{x}[L], \mathbf{s}, \mathbf{y}} \in \mathcal{D} \cap \mathcal{D}_{1}^{c} \cap \mathcal{D}_{3}^{c}} 2^{n\left(\left[R-I\left(\mathbf{x}_{i_{\tau}} ; \mathbf{x}, \mathbf{s} \mid \mathbf{u}\right)\right]^{+}+\varepsilon\right)} 2^{-n I\left(\mathbf{y} ; \mathbf{x}_{i_{\tau}} \mid \mathbf{u}, \mathbf{x}, \mathbf{s}\right)} .
\end{aligned}
$$

Inequality (37) follows from Equation (15). 
Since $\tau_{\mathbf{u}, \mathbf{x}, \mathbf{x}_{[L]}, \mathbf{s}, \mathbf{y}} \in \mathcal{D}_{1}^{c} \cap \mathcal{D}_{3}^{c}$,

$$
\varepsilon>I\left(\mathbf{x} ; \mathbf{x}_{i_{\tau}}, \mathbf{s} \mid \mathbf{u}\right)-\left[R-I\left(\mathbf{x}_{i_{\tau}} ; \mathbf{s} \mid \mathbf{u}\right)\right]^{+}=I\left(\mathbf{x} ; \mathbf{x}_{i_{\tau}}, \mathbf{s} \mid \mathbf{u}\right)-\left(R-I\left(\mathbf{x}_{i_{\tau}} ; \mathbf{s} \mid \mathbf{u}\right)\right) .
$$

Hence

$$
\begin{aligned}
& R>I\left(\mathbf{x} ; \mathbf{x}_{i_{\tau}}, \mathbf{s} \mid \mathbf{u}\right)+I\left(\mathbf{x}_{i_{\tau}} ; \mathbf{s} \mid \mathbf{u}\right)-\varepsilon=I\left(\mathbf{x} ; \mathbf{x}_{i_{\tau}} \mid \mathbf{u}, \mathbf{s}\right)+I(\mathbf{x} ; \mathbf{s} \mid \mathbf{u})+I\left(\mathbf{x}_{i_{\tau}} ; \mathbf{s} \mid \mathbf{u}\right)-\varepsilon \geqslant I\left(\mathbf{x}_{i_{\tau}} ; \mathbf{x}, \mathbf{s} \mid \mathbf{u}\right)-\varepsilon \\
\Longrightarrow & R-I\left(\mathbf{x}_{i_{\tau}} ; \mathbf{x}, \mathbf{s} \mid \mathbf{u}\right)+\varepsilon>0
\end{aligned}
$$

Consequently,

$$
\begin{aligned}
{\left[R-I\left(\mathbf{x}_{i_{\tau}} ; \mathbf{x}, \mathbf{s} \mid \mathbf{u}\right)\right]^{+} } & = \begin{cases}0, & R<I\left(\mathbf{x}_{i_{\tau}} ; \mathbf{x}, \mathbf{s} \mid \mathbf{u}\right) \\
R-I\left(\mathbf{x}_{i_{\tau}} ; \mathbf{x}, \mathbf{s} \mid \mathbf{u}\right), & R \geqslant I\left(\mathbf{x}_{i_{\tau}} ; \mathbf{x}, \mathbf{s} \mid \mathbf{u}\right)\end{cases} \\
& \leqslant \begin{cases}R-I\left(\mathbf{x}_{i_{\tau}} ; \mathbf{x}, \mathbf{s} \mid \mathbf{u}\right)+\varepsilon, & R<I\left(\mathbf{x}_{i_{\tau}} ; \mathbf{x}, \mathbf{s} \mid \mathbf{u}\right) \\
R-I\left(\mathbf{x}_{i_{\tau}} ; \mathbf{x}, \mathbf{s} \mid \mathbf{u}\right), & R \geqslant I\left(\mathbf{x}_{i_{\tau}} ; \mathbf{x}, \mathbf{s} \mid \mathbf{u}\right)\end{cases} \\
& \leqslant R-I\left(\mathbf{x}_{i_{\tau}} ; \mathbf{x}, \mathbf{s} \mid \mathbf{u}\right)+\varepsilon .
\end{aligned}
$$

Substituting Inequality (38) to Inequality (37), we continue the calculation.

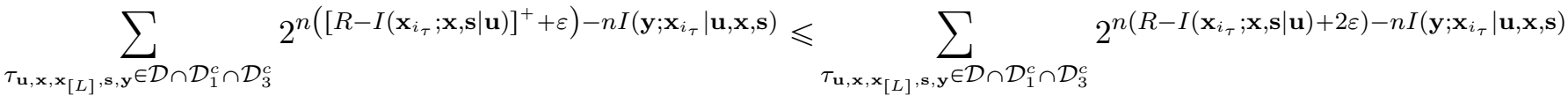

$$
\begin{aligned}
& =\sum_{\tau_{\mathbf{u}, \mathbf{x}, \mathbf{x}[L]}, \mathbf{s}, \mathbf{y} \in \mathcal{D} \cap \mathcal{D}_{1}^{c} \cap \mathcal{D}_{3}^{c}} 2^{-n I\left(\mathbf{x}_{i_{\tau}} ; \mathbf{x}, \mathbf{s}, \mathbf{y} \mid \mathbf{u}\right)+n R+2 n \varepsilon} \\
& =\sum_{\tau_{\mathbf{u}, \mathbf{x}, \mathbf{x}[L]}, \mathbf{s}, \mathbf{y} \in \mathcal{D} \cap \mathcal{D}_{1}^{c} \cap \mathcal{D}_{3}^{c}} 2^{-n\left(I\left(\mathbf{x}_{i_{\tau}} ; \mathbf{y} \mid \mathbf{u}\right)-R-2 \varepsilon\right)}
\end{aligned}
$$

\section{CONVERSE: CAPACITY UPPER BOUND}

In this section, we prove Theorem 14.

Fix any constant $\delta>0$. Fix any code $\mathcal{C}$ of rate $C_{L}\left(\mathcal{A}_{\text {obli }}\right)+\delta$. Let $M:=|\mathcal{C}|$. Without loss of generality (Lemma 7), assume that $\mathcal{C}$ is $\left(\lambda, \widehat{P}_{\mathbf{x}}\right)$-constant-composition for some constant $\lambda>0$ to be determined later and for some distribution $P_{\mathrm{x}} \in \Delta(\mathcal{X})$.

By Lemma 18, there exists a constant $\nu>0$ such that a $\nu$ fraction of types $\tau_{\underline{x}_{\mathcal{L}}}$ of ordered $L$-lists $\underline{x}_{\mathcal{L}}$, where $\mathcal{L} \in\left(\begin{array}{c}{[M]} \\ L\end{array}\right)$, are $\varepsilon$-close to $\mathrm{CP}^{\otimes L}\left(\widehat{P}_{\mathbf{x}}\right)$. By quantizing $\left(\mathrm{CP}^{\otimes L}\left(\widehat{P}_{\mathbf{x}}\right)\right)_{\varepsilon} \cap \Delta\left(\mathcal{X}^{L}\right)$, there is a smaller positive constant $0<c \leqslant \nu$ such that a $c$ fraction of types of ordered $L$-lists in $\mathcal{C}$ are $\eta$-close to $\widehat{P}_{\mathbf{x}_{1}, \cdots, \mathbf{x}_{L}}$ for some $\widehat{P}_{\mathbf{x}_{1}, \cdots, \mathbf{x}_{L}} \in$ $\left(\mathrm{CP}^{\otimes L}\left(\widehat{P}_{\mathbf{x}}\right)\right)_{\varepsilon} \cap \Delta\left(\mathcal{X}^{L}\right)$. Projecting $\widehat{P}_{\mathbf{x}_{1}, \cdots, \mathbf{x}}$ back to $\mathrm{CP}^{\otimes L}\left(\widehat{P}_{\mathbf{x}}\right)$, we finally get a $c$ fraction of order- $L$ types in $\mathcal{C}$ that are $(\eta+\varepsilon)$-close to $\widetilde{P}_{\mathbf{x}_{1}, \cdots, \mathbf{x}_{L}}$ for some $\widetilde{P}_{\mathbf{x}_{1}, \cdots, \mathbf{x}_{L}} \in \mathrm{CP}^{\otimes L}\left(\widehat{P}_{\mathbf{x}}\right)$.

Conjecture 21. Let $\mathcal{C} \subseteq \mathcal{X}^{n}$ be any $\left(\lambda, \widehat{P}_{\mathbf{x}}\right)$-constant-composition code of size $M$ where $M \geqslant|\mathcal{X}|^{n R}$ for some constant $R \in(0,1]$. Let $L \in \mathbb{Z}_{\geqslant 1}$. We know from the above argument that for any sufficiently small constant $\varepsilon>0$, there must exist a constant $c>0$ and a distribution $\widetilde{P}_{\mathbf{x}_{1}, \cdots, \mathbf{x}_{L}} \in \mathrm{CP}^{\otimes L}\left(\widehat{P}_{\mathbf{x}}\right)$ such that a c fraction of $\tau_{\underline{x}_{\mathcal{L}}}\left(\mathcal{L} \in\left(\begin{array}{c}{[M]} \\ L\end{array}\right)\right)$ satisfy $d\left(\tau_{\underline{x}_{\mathcal{L}}}, \widetilde{P}_{\mathbf{x}_{1}, \cdots, \mathbf{x}_{L}}\right) \leqslant \varepsilon$. Assume that the CP-distribution can be decomposed as $\widetilde{P}_{\mathbf{x}_{1}, \cdots, \mathbf{x}_{L}}=\left[\widetilde{P}_{\mathbf{u}} \widetilde{P}_{\mathbf{x} \mid \mathbf{u}}\right]_{\mathbf{x}_{1}, \cdots, \mathbf{x}_{L}}$ for some $k \in \mathbb{Z}_{\geqslant 1}, P_{\mathbf{u}} \in \Delta(\mathcal{U})(\mathcal{U}=[k])$ and $P_{\mathbf{x} \mid \mathbf{u}} \in \Delta(\mathcal{X} \mid \mathcal{U})$. It is conjectured that for any sufficiently small constant $\gamma>0$, there exist a constant $\mu>0$, a subcode $\mathcal{C}^{\prime} \subset \mathcal{C}$ of size $\left|\mathcal{C}^{\prime}\right|=M^{\prime} \geqslant \mu M$ and a universal time-sharing sequence $\underline{u} \in \mathcal{U}^{n}$ for $\mathcal{C}^{\prime}$ such that $d\left(\tau_{\underline{u}, \underline{x}}, \widetilde{P}_{\mathbf{u}} \widetilde{P}_{\mathbf{x} \mid \mathbf{u}}\right) \leqslant \gamma$ for every $\underline{x} \in \mathcal{C}^{\prime}$.

\section{A. James's jamming strategy}

Let $\mathcal{C}=\left\{\underline{x}_{i}\right\}_{i \in[M]}$ be a $\left(\lambda, \widehat{P}_{\mathbf{x}}\right)$-constant-composition code of size $M$. Let $\delta_{L}, \gamma$ be positive constants to be determined later.

1) Construct a $\delta_{L}$-net $\mathcal{N}_{L}$ of $\mathrm{CP}^{\otimes L}\left(\widehat{P}_{\mathbf{x}}\right)$. 
2) Draw all types $\tau_{\underline{x}_{\mathcal{C}}}\left(\mathcal{L} \in\left({ }^{[M]}{ }_{L}\right)\right.$ ) on the space $\Delta\left(\mathcal{X}^{L}\right)$. (Note that the types may not fall within $\mathrm{CP}^{\otimes L}\left(\widehat{P}_{\mathbf{x}}\right)$.) Find the Voronoi cell induced by $\mathcal{N}_{L}$ that contains the largest number of types of ordered $L$-tuples in $\mathcal{C}$. Let $\widetilde{P}_{\mathbf{x}_{1}, \cdots, \mathbf{x}_{L}}$ denote the representative of that cell. Let $\left(\widetilde{P}_{\mathbf{u}}, \widetilde{P}_{\mathbf{x} \mid \mathbf{u}}\right)$ be a CP-decomposition of $\widetilde{P}_{\mathbf{x}_{1}, \cdots, \mathbf{x}_{L}}$. Let $\mathcal{C}^{\prime} \subseteq \mathcal{C}$ denote the set of all codewords in the Voronoi cell corresponding to $\widetilde{P}_{\mathbf{x}_{1}, \cdots, \mathbf{x}_{L}}$.

3) Find the largest subsubcode $\mathcal{C}^{\prime \prime} \subseteq \mathcal{C}^{\prime}$ such that there is a sequence $\underline{u} \in \mathcal{U}^{n}$ satisfying $d\left(\tau_{\underline{u}, \underline{x}}, \widetilde{P}_{\mathbf{u}}, \widetilde{P}_{\mathbf{x} \mid \mathbf{u}}\right) \leqslant \gamma$ for all $\underline{x} \in \mathcal{C}^{\prime \prime}$.

4) Sample $\underline{\mathbf{s}} \in \mathcal{S}^{n}$ according to the following distribution

$$
\underline{\mathbf{s}} \sim \prod_{j=1}^{n} U_{\mathbf{s} \mid \mathbf{u}=\underline{u}(j)}=: U_{\underline{\mathbf{s}} \mid \underline{u}} .
$$

\section{B. Analysis of average error probability}

Let $\psi: \mathcal{Y}^{n} \rightarrow[M]$ be the $L$-list-decoder equipped with $\mathcal{C}$. By Definition $2,|\psi(\underline{y})| \leqslant L$ for any $\underline{y} \in \mathcal{Y}^{n}$. Using Definition 3, we compute the expected average error probability over the random jamming sequence generation defined by Equation (40).

$$
\begin{aligned}
& \underset{\underline{\mathbf{s}} \sim U_{\underline{\mathbf{s}} \mid \underline{u}}}{\mathbb{E}}\left[P_{\mathrm{e}, \operatorname{avg}}(\underline{\mathbf{s}})\right]=\frac{1}{M} \sum_{i \in[M]} \underset{\underline{\mathbf{s}} \sim U_{\underline{\underline{s}} \mid \underline{u}}}{\mathbb{E}}\left[P_{\mathrm{e}}(i, \underline{\mathbf{s}})\right] \\
& =\frac{1}{M} \sum_{i \in[M]} \sum_{\underline{y} \in \mathcal{Y}^{n}: \psi(\underline{y}) \nexists i} \underset{\underline{\mathbf{s}} \sim U_{\underline{\mathbf{s}} \mid \underline{u}}}{\mathbb{E}}\left[W_{\mathbf{y} \mid \mathbf{x}, \mathbf{s}}^{\otimes n}\left(\underline{y} \mid \underline{x}_{i}, \underline{\mathbf{s}}\right)\right] \\
& =\frac{1}{M} \sum_{i \in[M]} \sum_{y \in \mathcal{Y}^{n}: \psi(\underline{y}) \nexists i} \prod_{j=1}^{n} \underset{\mathbf{s}(j) \sim U_{\mathbf{s} \mid \mathbf{u}=\underline{u}(j)}}{\mathbb{E}}\left[W_{\mathbf{y} \mid \mathbf{x}, \mathbf{s}}\left(\underline{y}(j) \mid \underline{x}_{i}(j), \underline{\mathbf{s}}(j)\right)\right] .
\end{aligned}
$$

We observe that Equation (41) can be viewed as the average error probability of a time-varying channel $\widetilde{W}_{\mathbf{y} \mid \mathbf{x}, \mathbf{u}}$ defined according to

$$
\widetilde{W}_{\mathbf{y} \mid \mathbf{x}, \mathbf{u}}(y \mid x, u):=\underset{\mathbf{s} \sim U_{\mathbf{s} \mid \mathbf{u}=u}}{\mathbb{E}}\left[W_{\mathbf{y} \mid \mathbf{x}, \mathbf{s}}(y \mid x, s)\right]=\sum_{s \in \mathcal{S}} U_{\mathbf{s} \mid \mathbf{u}}(s \mid u) W_{\mathbf{y} \mid \mathbf{x}, \mathbf{s}}(y \mid x, s),
$$

for all $y \in \mathcal{Y}, x \in \mathcal{X}, u \in \mathcal{U}$. Under this definition, Equation (41) can be written as

$$
\begin{aligned}
\text { Equation (41) } & =\frac{1}{M} \sum_{i \in[M]} \sum_{\underline{y} \in \mathcal{Y}^{n}: \psi(\underline{y}) \nexists i} \prod_{j=1}^{n} \widetilde{W}_{\mathbf{y} \mid \mathbf{x}, \mathbf{u}}\left(\underline{y}(j) \mid \underline{x}_{i}(j), \underline{u}(j)\right) \\
& =\frac{1}{M} \sum_{i \in[M] \underline{y} \in \underline{\mathcal{Y}}^{n}: \psi(\underline{y}) \nexists i} \int_{W_{\mathbf{y} \mid \mathbf{x}, \mathbf{u}}^{\otimes n}}\left(\underline{y} \mid \underline{x}_{i}, \underline{u}\right)=\widetilde{P}_{\mathrm{e}, \operatorname{avg}}(\mathcal{C}),
\end{aligned}
$$

where $\widetilde{P}_{\mathrm{e}, \text { avg }}$ is the average error probability of $\mathcal{C}$ used over $\widetilde{W}_{\mathbf{y} \mid \mathbf{x}, \mathbf{u}}^{\otimes n}$. To apply strong converse for fading channels with approximate constant-composition codes and list-decoding, we massage Equation (42) as follows.

$$
\begin{aligned}
\text { Equation (42) } & \geqslant \frac{1}{M} \sum_{i \in[M]: \underline{x}_{i} \in \mathcal{C}^{\prime \prime}} \sum_{\underline{y} \in \mathcal{Y}^{n}: \psi(\underline{y}) \nexists i} \widetilde{W}_{\mathbf{y} \mid \mathbf{x}, \mathbf{u}}^{\otimes n}\left(\underline{y} \mid \underline{x}_{i}, \underline{u}\right) \\
& \geqslant \frac{1}{M} \sum_{i \in[M]: \underline{x}_{i} \in \mathcal{C}^{\prime \prime}} \sum_{\underline{y} \in \mathcal{Y}^{n}: \psi_{\mathrm{opt}}^{\prime \prime}(\underline{y}) \nexists i} \widetilde{W}_{\mathbf{y} \mid \mathbf{x}, \mathbf{u}}^{\otimes n}\left(\underline{y} \mid \underline{x}_{i}, \underline{u}\right) \\
& =\frac{M^{\prime \prime}}{M} \frac{1}{M^{\prime \prime}} \sum_{i \in[M]: \underline{x}_{i} \in \mathcal{C}^{\prime \prime}} \sum_{\underline{y} \in \mathcal{Y}^{n}: \psi_{\mathrm{opt}}^{\prime \prime}(\underline{y}) \nexists i} \widetilde{W}_{\mathbf{y} \mid \mathbf{x}, \mathbf{u}}^{\otimes n}\left(\underline{y} \mid \underline{x}_{i}, \underline{u}\right),
\end{aligned}
$$

where in Equation (44), $\left|\mathcal{C}^{\prime \prime}\right|$ is denoted by $M^{\prime \prime}$ and in Equation (43) we use $\psi_{\mathrm{opt}}^{\prime \prime}: \mathcal{Y}^{n} \rightarrow\left(\begin{array}{c}{\left[M^{\prime \prime}\right]} \\ \leqslant L\end{array}\right)$ to denote the optimal $L$-list-decoder for $\mathcal{C}^{\prime \prime}$ used over $\widetilde{W}_{\mathbf{y} \mid \mathbf{x}, \mathbf{u}}^{\otimes n}$.

By Equation (10), $M^{\prime} / M \geqslant c$. By Conjecture 21, $M^{\prime \prime} / M^{\prime} \geqslant \mu$. Therefore we have

$$
\frac{M^{\prime \prime}}{M}=\frac{M^{\prime}}{M} \frac{M^{\prime \prime}}{M^{\prime}} \geqslant c \mu>0,
$$


which is a positive constant. Note that

$$
\widetilde{P}_{\mathrm{e}, \mathrm{avg}}\left(\mathcal{C}^{\prime \prime}\right):=\frac{1}{M^{\prime \prime}} \sum_{i \in[M]: \underline{x}_{i} \in \mathcal{C}^{\prime \prime}} \sum_{\underline{y} \in \mathcal{Y}^{n}: \psi_{\mathrm{opt}}^{\prime \prime}(\underline{y}) \nexists i} \widetilde{W}_{\mathbf{y} \mid \mathbf{x}, \mathbf{u}}^{\otimes n}\left(\underline{y} \mid \underline{x}_{i}, \underline{u}\right)
$$

is the average error probability of $\mathcal{C}^{\prime \prime}$ used over $\widetilde{W}_{\mathbf{y} \mid \mathbf{x}, \mathbf{u}}^{\otimes n}$ under the optimal $L$-list-decoder. We are going to apply the strong converse bound on $\widetilde{P}_{\mathrm{e}, \text { avg }}\left(\mathcal{C}^{\prime \prime}\right)$. To this end, first recall the capacity of fading channels.

Theorem 22 (Capacity of fading DMCs, Chap. 23 [EGK11]). Let $W_{\mathbf{y} \mid \mathbf{x}, \mathbf{u}}$ be a fading channel with fast block fading according to a fixed sequence $\underline{u} \in \mathcal{U}^{n}$ (where $|\mathcal{U}|$ is a constant) of type $P_{\mathbf{u}}{ }^{12}$ The capacity of $W_{\mathbf{y} \mid \mathbf{x}, \mathbf{u}}$ is given by

$$
C\left(W_{\mathbf{y} \mid \mathbf{x}, \mathbf{u}}\right)=\max _{P_{\mathbf{x} \mid \mathbf{u} \in \Delta}(\mathcal{X} \mid \mathcal{U})} I(\mathbf{x} ; \mathbf{y} \mid \mathbf{u}),
$$

where the mutual information is evaluated according to $P_{\mathbf{u}} P_{\mathbf{x} \mid \mathbf{u}} W_{\mathbf{y} \mid \mathbf{x}, \mathbf{u}}$.

The strong converse result for fading channels under list-decoding with approximate constant-composition codes follows from standard techniques. We state the result below and prove it in Appendix E.

Theorem 23 (Strong converse for fading DMCs with approximate constant-composition codes and list-decoding). Let $W_{\mathbf{y} \mid \mathbf{x}, \mathbf{u}}$ be a fading DMC with fast fading according to a sequence $\underline{u} \in \mathcal{U}^{n}$ of type $P_{\mathbf{u}} \in \Delta(\mathcal{U})$ where $|\mathcal{U}|$ is a constant. Fix any constants $\delta>0$ and $0<\lambda \ll \delta$. Let $P_{\mathbf{x} \mid \mathbf{u}} \in \Delta(\mathcal{X} \mid \mathcal{U})$. For any list-size $L \in \mathbb{Z}_{\geqslant 1}$ and any $\left(\lambda, \underline{u}, P_{\mathbf{x} \mid \mathbf{u}}\right)$-constant-composition code $\mathcal{C}$ of rate $R=\frac{1}{n} \log \frac{|\mathcal{C l}|}{L} \geqslant C\left(W_{\mathbf{y} \mid \mathbf{x}, \mathbf{u}}\right)+\delta$ (where $C\left(W_{\mathbf{y} \mid \mathbf{x}, \mathbf{u}}\right)$ is defined in Equation (46)), the average error probability of $\mathcal{C}$ used over $W_{\mathbf{y} \mid \mathbf{x}, \mathbf{u}}^{\otimes n}$ is approaching 1. More precisely

$$
P_{\mathrm{e}, \mathrm{avg}}(\mathcal{C}) \geqslant 1-2^{-n f(\delta, \lambda)}
$$

for some function $f(\delta, \lambda)>0$ satisfying $f(\delta, \lambda) \stackrel{\delta, \lambda \rightarrow 0}{\longrightarrow} 0$.

By Theorem 23,

$$
\widetilde{P}_{\mathrm{e}, \mathrm{avg}}\left(\mathcal{C}^{\prime \prime}\right) \geqslant 1-2^{-n f(\delta, \lambda)} .
$$

Finally, plugging Equation (45) and Equation (48) into Equation (44), we get

$$
\underset{\underline{\mathbf{s}} \sim U_{\underline{\mathbf{s}} \mid \underline{u}}^{\mathbb{E}}}{\mathbb{E}}\left[P_{\mathrm{e}, \operatorname{avg}}(\underline{\mathbf{s}})\right] \geqslant c \mu\left(1-2^{-n f(\delta, \lambda)}\right) \stackrel{n \rightarrow \infty}{\longrightarrow} c \mu>0,
$$

which completes the error analysis.

\section{A subcode extraction procedure towards Conjecture 21}

Towards a tight capacity characterization, we propose a natural subcode extraction procedure. However, we do not know how to prove that it gives rise to a matching capacity upper bound. The procedure is described below. Given any $\left(\lambda, P_{\mathbf{x}}\right)$-constant-composition code $\mathcal{C} \subseteq \mathcal{X}^{n}$ of size $M$. We extract a subcode $\mathcal{C}^{\prime} \subseteq \mathcal{C}$ via the following steps. Let $\lambda^{\prime}>0$ be a constant to be determined.

1) Quantize $\Delta(\mathcal{X})$ using a $\zeta$-net $\mathcal{N}=\left\{P_{\mathbf{x}_{i}}\right\}_{i \in[N]}$ (where $P_{\mathbf{x}_{i}} \in \Delta(\mathcal{X})$ for each $i$ ) of size $N$ at most $N(\zeta,|\mathcal{X}|)$ (by Lemma 6).

2) Cluster the columns of $\mathcal{C} \in \mathcal{X}^{M \times n}$ according their types. This naturally induces a time-sharing sequence $\underline{u} \in \mathcal{U}^{n}$ defined as follows. The alphabet $\mathcal{U}$ has size at most $N$. The $j$-th $(j \in[n])$ component $\underline{u}(j)$ is set $i \in[N]$ if the $j$-th column of $\mathcal{C}$ has type $\zeta$-close to $P_{\mathbf{x}_{i}} \in \mathcal{N}$.

3) Find the largest subcode $\mathcal{C}^{\prime} \subseteq \mathcal{C}$ with the following properties.

a) The subcode $\mathcal{C}^{\prime}$ has size lat least $\theta M$ for some constant $\theta>0$.

\footnotetext{
${ }^{12}$ That is, the fading process $\left\{\mathbf{u}_{i}\right\}_{i \in[n]}$ is a constant $u \in \mathcal{U}$ in each coherence time interval $\{i \in[n]: \underline{u}(i)=u\}$. Since $|\mathcal{U}| \ll n$ is a constant, this model is called fast fading.
} 
b) For each $i \in \mathcal{U}$, the shorter subcode $\left.\mathcal{C}^{\prime}\right|_{i} \subseteq \mathcal{X}^{n \tau_{\underline{u}}(i)}$ is $\left(\lambda^{\prime}, P_{\mathbf{x}_{i}}^{\prime}\right)$-constant-composition for some distribution $P_{\mathbf{x}_{i}}^{\prime} \in \Delta(\mathcal{X})^{13}$. Here $\left.\mathcal{C}^{\prime}\right|_{i} \subseteq \mathcal{X}^{n \tau_{\underline{u}}(i)}$ denotes the puncturing of $\mathcal{C}^{\prime}$ onto the components $j$ 's $(j \in[n])$ such that $\underline{u}(j)=i$.

c) The subcode $\mathcal{C}^{\prime}$ is the one that minimizes the induced conditional mutual information

$$
\max _{U_{\mathbf{s} \mid \mathbf{u}} \in \Delta(\mathcal{S} \mid \mathcal{U}):\left[P_{\mathbf{u}} U_{\mathbf{s} \mid \mathbf{u}}\right]_{\mathbf{s}} \in \lambda_{\mathbf{s}}} I(\mathbf{x} ; \mathbf{y} \mid \mathbf{u})
$$

among all subcodes satisfying Items $3 \mathrm{a}$ and $3 \mathrm{~b}$, where the conditional mutual information is evaluated according to $P_{\mathbf{u}, \mathbf{x}, \mathbf{y}}(u, x, y)=\tau_{\underline{u}}(u) P_{\mathbf{x}_{u}}^{\prime}(x) U_{\mathbf{s} \mid \mathbf{x}}(s \mid x) W_{\mathbf{y} \mid \mathbf{x}, \mathbf{s}}(y \mid x, s)$.

We claim that subcodes satisfying Items $3 a$ and $3 b$ do exist for sufficiently small $\theta$. Indeed, they can be constructed as follows. By approximate-constant-composition reduction (Lemma 7), for $i=1$, we can find a subcode $\mathcal{C}_{1}^{\prime} \subseteq \mathcal{C}$ of size $M_{1} \geqslant \theta_{0} M$ where $\theta_{0} \geqslant\left(\frac{|\mathcal{X}|}{2 \lambda^{\prime}}+1\right)^{-|\mathcal{X}|}$ such that $\left.\mathcal{C}_{1}^{\prime}\right|_{1} \subseteq \mathcal{X}^{n \tau_{\underline{u}}(1)}$ is $\left(\lambda^{\prime}, P_{\mathbf{x}_{1}}^{\prime}\right)$-constant-composition for some $P_{\mathbf{x}_{1}}^{\prime} \in \Delta(\mathcal{X})$. For $i=2$, there exists a subcode $\mathcal{C}_{2}^{\prime} \subseteq \mathcal{C}_{1}^{\prime}$ of size $M_{2} \geqslant \theta_{0} M_{1} \geqslant \theta_{0}^{2} M$ such that $\left.\mathcal{C}_{2}^{\prime}\right|_{2} \subseteq \mathcal{X}^{n \tau_{\underline{u}}(2)}$ is $\left(\lambda^{\prime}, P_{\mathbf{x}_{2}}^{\prime}\right)$-constant-composition for some $P_{\mathbf{x}_{2}}^{\prime} \in \Delta(\mathcal{X})$. Note that $\left.\mathcal{C}_{2}^{\prime}\right|_{1} \subseteq \mathcal{X}^{n \tau_{\underline{u}}}(1)$ is still $\left(\lambda^{\prime}, P_{\mathbf{x}_{1}}^{\prime}\right)$-constantcomposition since $\mathcal{C}_{2}^{\prime} \subseteq \mathcal{C}_{1}^{\prime}$. Iteratively doing this for each $i=1,2, \cdots,|\mathcal{U}|$, we end up with a subcode $\mathcal{C}^{\prime}=\mathcal{C}_{|\mathcal{U}|}^{\prime}$ of size at least $M_{|\mathcal{U}|} \geqslant \theta_{0}^{|\mathcal{U}|} M$ such that for each $i \in \mathcal{U},\left.\mathcal{C}^{\prime}\right|_{i}$ is $\left(\lambda^{\prime}, P_{\mathbf{x}_{i}}^{\prime}\right)$-constant-composition. See Figure 8. Therefore,

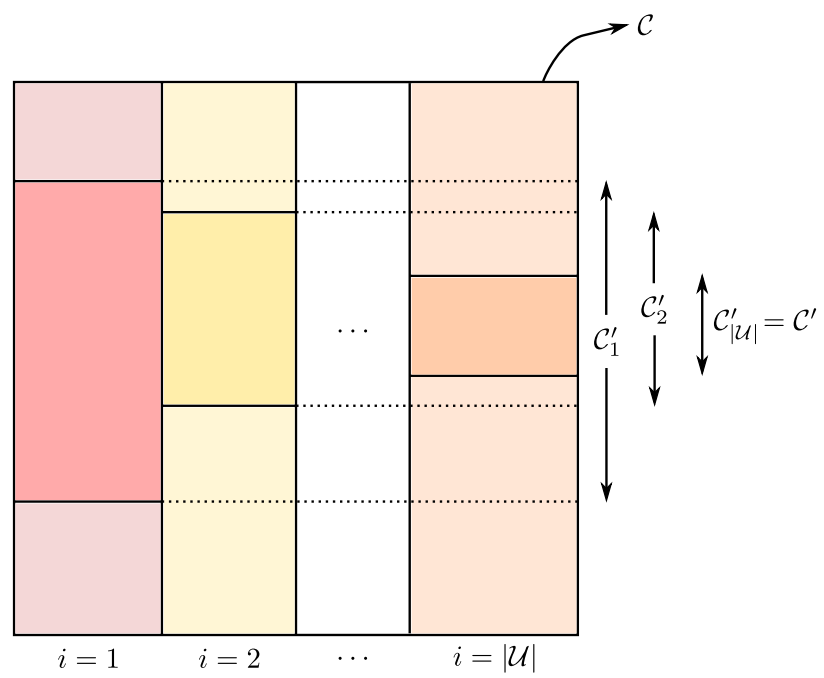

Fig. 8: A proposed procedure for extracting large subcodes that is chunk-wise constant-composition w.r.t. a timesharing sequence $\underline{u} \in \mathcal{U}^{n}$.

as long as $\theta \leqslant\left(\frac{|\mathcal{X}|}{2 \lambda^{\prime}}+1\right)^{-|\mathcal{X}| N} \leqslant \theta_{0}^{|\mathcal{U}|}$, subcodes satisfying Items $3 \mathrm{a}$ and $3 \mathrm{~b}$ can be found. The goal is to find the one with the minimum conditional mutual information.

By construction, a subcode $\mathcal{C}^{\prime}$ obtained as above is indeed constant-composition w.r.t. a universal time-sharing sequence $\underline{u}$ as desired in Conjecture 21. It is not hard to see that if a code $\mathcal{C}$ was truly sampled from the ensemble defined in Appendix $C$, then the above procedure will with high probability detect the correct underlying timesharing sequence $\underline{u}$ and the distributions $\left\{P_{\mathbf{x}_{u}}\right\}_{u \in \mathcal{U}}$ that $\mathcal{C}$ was sampled from. However, one crucial gap in the above construction that prevents us from proving a matching capacity upper bound is as follows. Recall that in the capacity expression (Equation (5)), the maximization is over non-L-symmetrizable CP-distributions $P_{\mathbf{x}_{[L]}}=$ $\sum_{u \in \mathcal{U}} P_{\mathbf{u}}(u) P_{\mathbf{x}_{u} L}^{\otimes L}$. Unfortunately, we do not know how to show the non- $L$-symmetrizability of the CP-distribution $\sum_{u \in \mathcal{U}} \tau_{\underline{u}}(u) P_{\mathbf{x}_{u}}^{\prime \otimes L}$ induced by the above construction.

\section{TECHNICAL REMARKS}

In the first two subsections (Sections XII-A and XII-B) of this section, we discuss different notions of symmetrizability for different channel models and their relations. See Figure 9 for an overview. In the last subsection

\footnotetext{
${ }^{13}$ Unless other operation is done otherwise, for each $i \in \mathcal{U}$, the column composition $P_{\mathbf{x}_{i}}$ may not be preserved in each punctured subcode $\mathcal{C}^{\prime}{ }_{i}$. This is why we only claim $\left(\lambda^{\prime}, P_{\mathbf{x}_{i}}^{\prime}\right)$-constant-composition for another distribution $P_{\mathbf{x}_{i}}^{\prime}$ that can be different from $P_{\mathbf{x}_{i}}$ a priori.
} 


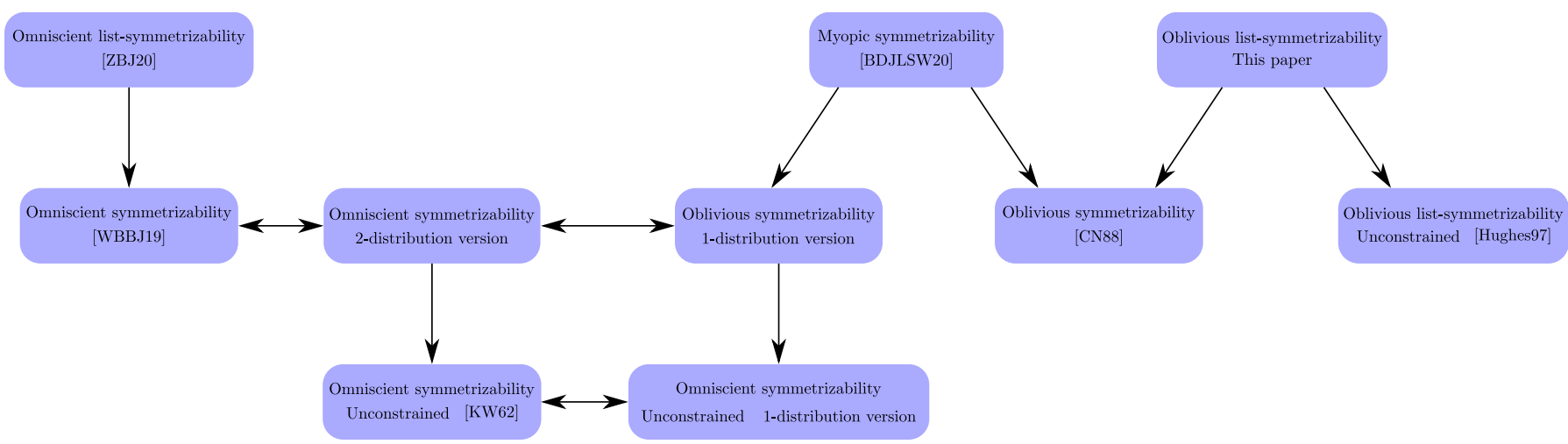

Fig. 9: Different notions of symmetrizability for different channel models. If there is an arrow $\rightarrow$ from Definition $A$ to Definition $B$, i.e., $A \rightarrow B$, then Definition $B$ is a special case of Definition $A$. If the arrow is bidirectional, it means that the definitions on two endpoints of the arrow are equivalent.

(Section XII-C), we discuss a seemingly more natural jamming strategy mentioned in Remark 10 for obtaining capacity upper bounds.

\section{A. Myopic/oblivious symmetrizability}

By the unique-decoding version of the generalized Plotkin bound [WBBJ19], in any sufficiently large approximately constant-composition code, there is a constant $c>0$ such that a $c$ fraction of types $\tau_{\underline{x}_{i}, \underline{x}_{j}}$ of ordered pairs $i<j$ of codewords is approximately CP. Curious readers may wonder why Csiszár-Narayan in their seminal paper [CN88b] did not resort to the heavy machinery in [WBBJ19] to prove a tight characterization of the capacity of input-constrained state-constrained oblivious AVCs. Before explaining the reason, we would like to first take a diversion and discuss different notions of symmetrizability/confusability in the AVC/adversarial channel literature.

Let us introduce myopic AVCs and omniscient AVCs. In oblivious AVCs, the adversary does not know which particular codeword from the codebook was transmitted, though he does know the codebook (which is known to everyone). Whereas in omniscient AVCs, the adversary knows precisely which codeword was transmitted.

Definition 18 (Omniscient AVCs). An omniscient $\mathrm{AVC} \mathcal{A}_{\mathrm{omni}}=\left(\mathcal{X}, \mathcal{S}, \mathcal{Y}, \lambda_{\mathbf{x}}, \lambda_{\mathbf{s}}, W_{\mathbf{y} \mid \mathbf{x}, \mathbf{s}}\right)$ consists of three alphabets $\mathcal{X}, \mathcal{S}, \mathcal{Y}$ for the input, jamming and output sequences, respectively; input constraints $\lambda_{\mathrm{x}} \subseteq \Delta(\mathcal{X})$ and state constraints $\lambda_{\mathbf{s}} \subseteq \Delta(\mathcal{S})$; an adversarial channel $W_{\mathbf{y} \mid \mathbf{x}, \mathbf{s}}$ from Alice to Bob governed by James. Knowing the codebook $\mathcal{C}$, Alice's encoder $\phi$, Bob's decoder $\psi$, receiving Alice's transmitted sequence $\underline{\mathbf{x}}$, James generates a jamming sequence $\underline{\mathbf{s}}$ and sends it through the channel $W_{\mathbf{y} \mid \mathbf{x}, \mathbf{s}}$. See Figure 10 for a block diagram of the omniscient AVC model under list-decoding. WHen $L=1$, it degenerates to the unique-decoding case.

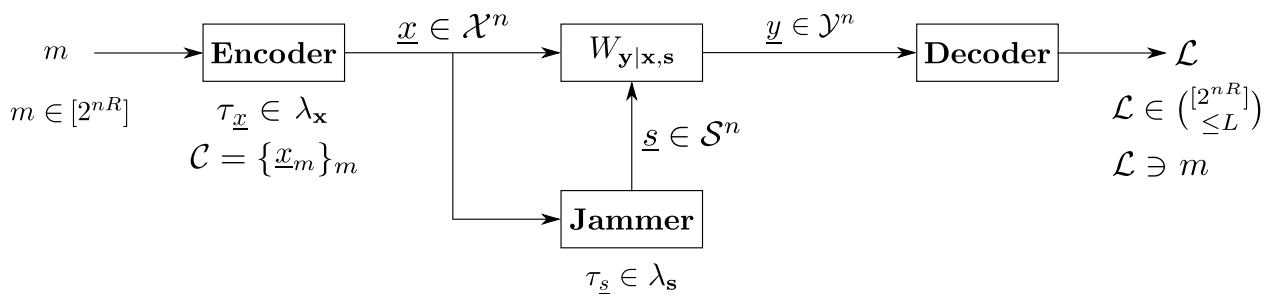

Fig. 10: Block diagram of an omniscient AVC under list-decoding.

The myopic AVCs are a natural information-theoretic bridge between the oblivious and omniscient models. In the myopic setting, the adversary observes a noisy version of the transmitted sequence through a $D M C$.

Definition 19 (Myopic AVCs). A myopic AVC $\mathcal{A}_{\text {myop }}=\left(\mathcal{X}, \mathcal{S}, \mathcal{Y}, \lambda_{\mathbf{x}}, \lambda_{\mathbf{s}}, W_{\mathbf{z} \mid \mathbf{x}}, W_{\mathbf{y} \mid \mathbf{x}, \mathbf{s}}\right)$ consists of three alphabets $\mathcal{X}, \mathcal{S}, \mathcal{Y}$ for the input, jamming and output sequences, respectively; input constraints $\lambda_{\mathbf{x}} \subseteq \Delta(\mathcal{X})$ and state constraints $\lambda_{\mathbf{s}} \subseteq \Delta(\mathcal{S})$; a DMC $W_{\mathbf{z} \mid \mathbf{x}}$ from Alice to James; an adversarial channel $W_{\mathbf{y} \mid \mathbf{x}, \mathbf{s}}$ from Alice to Bob governed by James. 
Knowing the codebook $\mathcal{C}$, Alice's encoder $\phi$, Bob's decoder $\psi$, receiving a noisy version $\underline{\mathbf{z}}$ of Alice's transmitted sequence $\underline{\mathbf{x}}$, James generates a jamming sequence $\underline{\mathbf{s}}$ and sends it through the channel $W_{\mathbf{y} \mid \mathbf{x}, \mathbf{s}}$. See Figure 11 for a block diagram of the myopic AVC model.

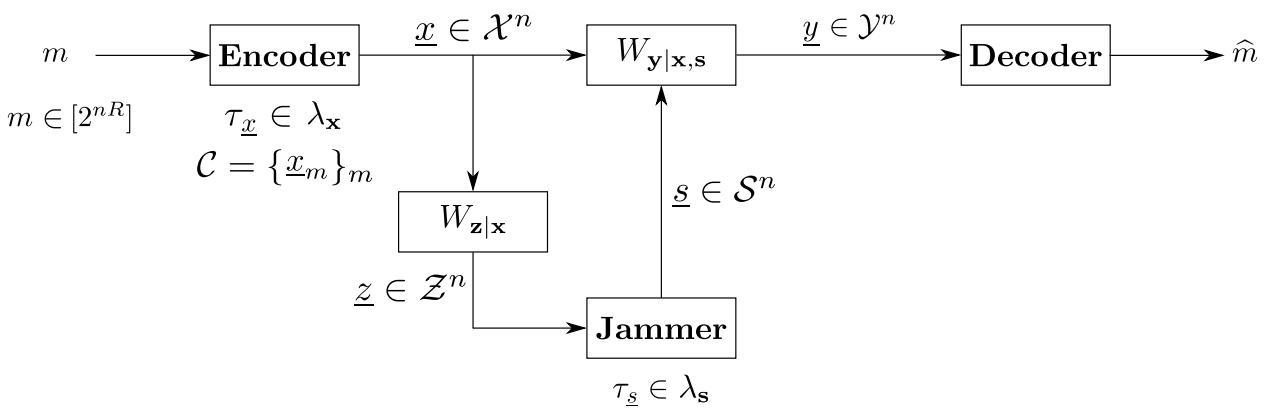

Fig. 11: Block diagram of a myopic AVC.

In a recent work $\left[\mathrm{BDJ}^{+} 20\right]$, a new notion of myopic symmetrizability was introduced.

Definition 20 (Myopically symmetrizing distributions). Define the set of myopically symmetrizing distributions as

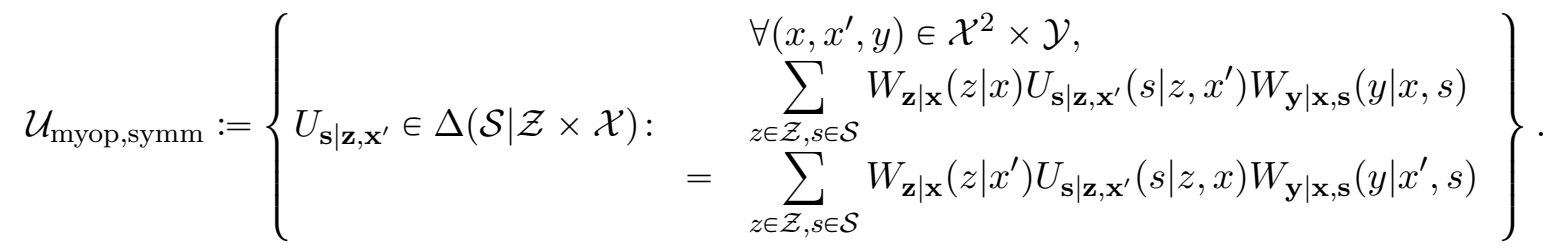

Definition 21 (Myopic symmetrizability, [BDJ $\left.{ }^{+} 20\right]$ ). Consider a myopic AVC $\mathcal{A}_{\text {myop }}$. An input distribution $P_{\mathbf{x}} \in$ $\lambda_{\mathbf{x}}$ is called myopically symmetrizable if for all $P_{\mathbf{x}, \mathbf{x}^{\prime}} \in \operatorname{CP}\left(P_{\mathbf{x}}\right)$ there is a $U_{\mathbf{s} \mid \mathbf{z}, \mathbf{x}^{\prime}} \in \mathcal{U}_{\text {myop,symm such that }}$ $\left[P_{\mathbf{x}, \mathbf{x}^{\prime}} W_{\mathbf{z} \mid \mathbf{x}} U_{\mathbf{s} \mid \mathbf{z}, \mathbf{x}^{\prime}}\right]_{\mathbf{s}} \in \lambda_{\mathbf{s}}$.

It was shown in $\left[\mathrm{BDJ}^{+} 20\right]$ that myopic symmetrizability is a sufficient (though not known to be necessary) condition for a myopic AVC to have zero rate.

Theorem 24 (Capacity positivity of myopic AVCs, , [BDJ' 20]). For any myopic AVC $\mathcal{A}_{\mathrm{myop}}$, the capacity is zero if every $P_{\mathbf{x}} \in \lambda_{\mathbf{x}}$ is myopically symmetrizable.

We claim that Csiszár-Narayan's notion of oblivious symmetrizability (for unique-decoding) is a special case of Definition 21. Indeed, the myopic model collapses to the oblivious model if $\mathbf{z} \Perp \mathbf{x}$. That is, the channel $W_{\mathbf{z} \mid \mathbf{x}}$ from Alice to James is infinitely noisy: $W_{\mathbf{z} \mid \mathbf{x}}=W_{\mathbf{z}}$. Taking $U_{\mathbf{s} \mid \mathbf{x}^{\prime}}\left(s \mid x^{\prime}\right):=\sum_{z \in \mathcal{Z}} W_{\mathbf{z}}(z) U_{\mathbf{s} \mid \mathbf{z}, \mathbf{x}^{\prime}}\left(s \mid z, x^{\prime}\right)$, the definition of $\mathcal{U}_{\text {myop,symm becomes: }}$

Definition 22 (Obliviously symmetrizing distributions). The set of obliviously symmetrizing distributions is defined as

$\mathcal{U}_{\text {obli,symm }}:=\left\{U_{\mathbf{s} \mid \mathbf{x}^{\prime}} \in \Delta(\mathcal{S} \mid \mathcal{X}): \forall\left(x, x^{\prime}, y\right) \in \mathcal{X}^{2} \times \mathcal{Y}, \sum_{s \in \mathcal{S}} U_{\mathbf{s} \mid \mathbf{x}^{\prime}}\left(s \mid x^{\prime}\right) W_{\mathbf{y} \mid \mathbf{x}, \mathbf{s}}(y \mid x, s)=\sum_{s \in \mathcal{S}} U_{\mathbf{s} \mid \mathbf{x}}(s \mid x) W_{\mathbf{y} \mid \mathbf{x}, \mathbf{s}}\left(y \mid x^{\prime}, s\right)\right\}$.

Note also that

$$
\begin{aligned}
{\left[P_{\mathbf{x}, \mathbf{x}^{\prime}} W_{\mathbf{z}} U_{\mathbf{s} \mid \mathbf{z}, \mathbf{x}^{\prime}}\right]_{\mathbf{S}}(s) } & =\sum_{\left(x, x^{\prime}\right) \in \mathcal{X}^{2}} \sum_{z \in \mathcal{Z}} P_{\mathbf{x}, \mathbf{x}^{\prime}}\left(x, x^{\prime}\right) W_{\mathbf{z}}(z) U_{\mathbf{s} \mid \mathbf{z}, \mathbf{x}^{\prime}}(s \mid z, x) \\
& =\sum_{\left(x, x^{\prime}\right) \in \mathcal{X}^{2}} P_{\mathbf{x}, \mathbf{x}^{\prime}}\left(x, x^{\prime}\right) U_{\mathbf{s} \mid \mathbf{x}^{\prime}}(s \mid x) \\
& =\sum_{x \in \mathcal{X}} P_{\mathbf{x}^{\prime}}(x) U_{\mathbf{s} \mid \mathbf{x}^{\prime}}(s \mid x) \\
& =\left[P_{\mathbf{x}} U_{\mathbf{s} \mid \mathbf{x}^{\prime}}\right]_{\mathbf{S}}(s)
\end{aligned}
$$


where Equation (49) follows since $\left[P_{\mathbf{x}, \mathbf{x}^{\prime}}\right]_{\mathbf{x}}=\left[P_{\mathbf{x}, \mathbf{x}^{\prime}}\right]_{\mathbf{x}^{\prime}}=P_{\mathbf{x}}$.

Therefore, we get the following notion of oblivious symmetrizability which precisely matches the one in [CN88b].

Definition 23 (Oblivious symmetrizability, [CN88b]). Given an oblivious AVC $\mathcal{A}_{\mathrm{obli}}$, an input distribution $P_{\mathbf{x}} \in \lambda_{\mathbf{x}}$ is called obliviously symmetrizable if there is a $U_{\mathbf{s} \mid \mathbf{x}^{\prime}} \in \mathcal{U}_{\text {obli,symm }}$ such that $\left[P_{\mathbf{x}} U_{\mathbf{s} \mid \mathbf{x}^{\prime}}\right]_{\mathbf{s}} \in \lambda_{\mathbf{s}}$.

It was shown in [CN88b] that:

Theorem 25 (Capacity positivity of oblivious AVCs, [CN88b]). An oblivious AVC $\mathcal{A}_{\mathrm{obli}}$ has zero capacity if and only if every $P_{\mathbf{x}} \in \lambda_{\mathbf{x}}$ is obliviously symmetrizable.

We are now ready to answer the question at the beginning of this section. Due to the oblivious nature, the joint distribution $P_{\mathbf{x}, \mathbf{x}^{\prime}}$ is marginalized when we evaluate the jamming distribution $P_{\mathbf{s}} \cdot{ }^{14}$ As a result, only the marginal input distribution $P_{\mathbf{x}}$ rather than the completely positive joint distribution $P_{\mathbf{x}, \mathbf{x}^{\prime}}$ matters in the definition of oblivious symmetrizability. It is as if Alice's transmitted signal $\mathbf{x}$ and James's spoofing signal $\mathbf{x}^{\prime}$ are independent rather than jointly $\mathrm{CP}^{15}$, i.e., $P_{\mathbf{x}, \mathbf{x}^{\prime}}=P_{\mathbf{x}} P_{\mathbf{x}}^{\top}$. Indeed, it is provably known $[\mathrm{CN} 88 \mathrm{~b}]$ that the optimal symmetrization strategy samples a spoofing codeword $\underline{x}^{\prime}$ uniformly from the codebook, independent of the transmitted codeword $\underline{\mathbf{x}}$.

Similarly, for oblivious AVCs under $L$-list-decoding, the $L$-oblivious symmetrizability only depends on the size- $L$ marginal distribution $P_{\mathbf{x}_{1}, \cdots, \mathbf{x}_{L}} \in \mathrm{CP}^{\otimes L}\left(P_{\mathbf{x}}\right)$ of a spoofing $L$-list, rather than the full joint distribution $P_{\mathbf{x}, \mathbf{x}_{1}, \cdots, \mathbf{x}_{L}}$ of the transmitted signal and the spoofing list. It is easy to see that 1-oblivious symmetrizability (Definition 14) is equivalent to Csiszár-Narayan's notion of oblivious symmetrizability (Definition 23).

\section{B. Myopic/omniscient symmetrizability}

In this section, we discuss the relation between symmetrizability for myopic channels and confusability for omniscient channels.

The omniscient channel, as mentioned in the previous section (Section XII-A), is a more coding-theoretic model of communication. In the absence of constraints, i.e., $\lambda_{\mathbf{x}}=\Delta(\mathcal{X}), \lambda_{\mathbf{s}}=\Delta \mathcal{S}$, the right notion of confusability ${ }^{16}$ was proposed by Kiefer-Wolfowitz [KW62].

Definition 24 (Unconstrained omniscient symmetrizability, [KW62]). An unconstrained omniscient AVC

$$
\mathcal{A}_{\text {omni }}=\left(\mathcal{X}, \mathcal{S}, \mathcal{Y}, \Delta(\mathcal{X}), \Delta(\mathcal{S}), W_{\mathbf{y} \mid \mathbf{x}, \mathbf{s}}\right)
$$

is called omnisciently symmetrizable if for all $\left(x, x^{\prime}\right) \in \mathcal{X}^{2}$, there are distributions $U_{\mathbf{s} \mid x, x^{\prime}}, U_{\mathbf{s}^{\prime} \mid x, x^{\prime}} \in \Delta(\mathcal{S})^{17}$ such that for every $y \in \mathcal{Y}$,

$$
\sum_{s \in \mathcal{S}} U_{\mathbf{s} \mid x, x^{\prime}}(s) W_{\mathbf{y} \mid \mathbf{x}, \mathbf{s}}(y \mid x, s)=\sum_{s \in \mathcal{S}} U_{\mathbf{s}^{\prime} \mid x, x^{\prime}}(s) W_{\mathbf{y} \mid \mathbf{x}, \mathbf{s}}\left(y \mid x^{\prime}, s\right) .
$$

Before proceeding to state Kiefer-Wolfowitz's characterization of capacity positivity, we remark that one can slightly simplify Definition 24 by restricting to the case where $U_{\mathbf{s} \mid x, x^{\prime}}=U_{\mathbf{s} \mid x, x^{\prime}}$. Indeed, once we have (potentially different) symmetrizing distributions $U_{\mathbf{s} \mid x, x^{\prime}}$ and $U_{\mathbf{s} \mid x, x^{\prime}}$, the distribution $V_{\mathbf{s} \mid x, x^{\prime}}=\frac{1}{2} U_{\mathbf{s} \mid x, x^{\prime}}+\frac{1}{2} U_{\mathbf{s} \mid x, x^{\prime}}^{\prime}$ is also symmetrizing. This follows trivially from Equation (50). Note also that associated to each pair $\left(x, x^{\prime}\right)$ there is a distribution $U_{\mathbf{s} \mid x, x^{\prime}}$. All these distributions $\left\{U_{\mathbf{s} \mid x, x^{\prime}}\right\}_{\left(x, x^{\prime}\right) \in \mathcal{X}^{2}}$ together give us a conditional distribution $U_{\mathbf{s} \mid \mathbf{x}, \mathbf{x}^{\prime}} \in \Delta\left(\mathcal{S} \mid \mathcal{X}^{2}\right)$. Therefore, Definition 24 can be simplified as follows.

Definition 25 (Omnisciently symmetrizing distributions). Define the set of omnisciently symmetrizing distributions as

$$
\mathcal{U}_{\text {omni,symm }}:=\left\{\begin{array}{ll} 
& \forall\left(x, x^{\prime}, y\right) \in \mathcal{X}^{2} \times \mathcal{Y}, \\
U_{\mathbf{s} \mid \mathbf{x}, \mathbf{x}^{\prime}} \in \Delta\left(\mathcal{S} \mid \mathcal{X}^{2}\right): & \sum_{s \in \mathcal{S}} U_{\mathbf{s} \mid \mathbf{x}, \mathbf{x}^{\prime}}\left(s \mid x, x^{\prime}\right) W_{\mathbf{y} \mid \mathbf{x}, \mathbf{s}}(y \mid x, s)=\sum_{s \in \mathcal{S}} U_{\mathbf{s} \mid \mathbf{x}, \mathbf{x}^{\prime}}\left(s \mid x, x^{\prime}\right) W_{\mathbf{y} \mid \mathbf{x}, \mathbf{s}}\left(y \mid x^{\prime}, s\right)
\end{array}\right\} .
$$

\footnotetext{
${ }^{14}$ Note that the evaluation of $P_{\mathbf{s}}$ is the only place in the definition of symmetrizability where $P_{\mathbf{x}, \mathbf{x}^{\prime}}$ plays a role. The definition of $\mathcal{U}_{\mathrm{symm}}$ has nothing to do with $P_{\mathbf{x}, \mathbf{x}^{\prime}}$.

${ }^{15}$ Of course, product distributions are also $\mathrm{CP}$. The point here is that requiring generic $\mathrm{CP}$ correlation turns out to be unnecessary.

${ }^{16}$ Due to historical reasons, the condition for zero capacity is referred to as confusability. We follow this convention in zero-error information theory. However, in essence, one can view it as a notion of symmetrizability for omniscient channels.

${ }^{17}$ Since the symmetrizing distributions may depend on $\left(x, x^{\prime}\right)$, we emphasize this dependence using the notation for conditional distribution.
} 
Definition 26 (Unconstrained omniscient symmetrizability, one-distribution version). An unconstrained omniscient AVC $\mathcal{A}_{\text {omni }}$ is called omnisciently symmetrizable if $\mathcal{U}_{\mathrm{omni} \text {,symm }} \neq \varnothing$.

Theorem 26 (Capacity positivity of unconstrained omniscient AVCs, [KW62]). An unconstrained omniscient AVC $\mathcal{A}_{\text {omni }}$ has zero capacity if and only if it is omnisciently symmetrizable.

In the presence of (input and state) constraints, characterizing the condition for capacity positivity turns out to be significantly more challenging. Incorporating state constraints is one of the major obstacles and ideas that are fundamentally different from [KW62] are required. Indeed, a sufficient and necessary condition is not available in the literature until very recently [WBBJ19]. The proof, which is of combinatorial nature, is a significant generalization of the Plotkin bound in classical coding theory.

Definition 27 (Omniscient symmetrizability, [WBBJ19]). Given an omniscient AVC $\mathcal{A}_{\mathrm{omni}}$, an input distribution $P_{\mathbf{x}} \in \lambda_{\mathbf{x}}$ is called omnisciently symmetrizable if for every $P_{\mathbf{x}, \mathbf{x}^{\prime}} \in \operatorname{CP}\left(P_{\mathbf{x}}\right)$, there is a joint distribution $P:=$ $P_{\mathbf{x}, \mathbf{x}^{\prime}, \mathbf{s}, \mathbf{s}^{\prime}, \mathbf{y}} \in \Delta\left(\mathcal{X}^{2} \times \mathcal{S}^{2} \times \mathcal{Y}\right)$ such that

1) $[P]_{\mathbf{x}}=[P]_{\mathbf{x}^{\prime}}=P_{\mathbf{x}},[P]_{\mathbf{x}, \mathbf{s}, \mathbf{y}}=[P]_{\mathbf{x}, \mathbf{s}} W_{\mathbf{y} \mid \mathbf{x}, \mathbf{s}},[P]_{\mathbf{x}^{\prime}, \mathbf{s}^{\prime}, \mathbf{y}}=[P]_{\mathbf{x}^{\prime}, \mathbf{s}^{\prime}} W_{\mathbf{y} \mid \mathbf{x}, \mathbf{s}} ;$

2) $[P]_{\mathrm{S}},[P]_{\mathbf{s}^{\prime}} \in \lambda_{\mathbf{s}}$.

It was shown in [WBBJ19] that:

Theorem 27 (Capacity positivity of omniscient AVCs, [WBBJ19]). An omniscient AVC $\mathcal{A}_{\mathrm{omni}}$ has zero capacity if and only if every $P_{\mathbf{x}} \in \lambda_{\mathbf{x}}$ is omnisciently symmetrizable.

Though having a seemingly different form, Definition 27 actually has an intimate relation with Definition 21. We will massage Definition 27 and argue that it is a special case of Definition 21. The condition in Item 1 can be rewritten as: for all $\left(x, x^{\prime}, s, s^{\prime}, y\right) \in \mathcal{X}^{2} \times \mathcal{S}^{2} \times \mathcal{Y}$,

$$
\begin{aligned}
P_{\mathbf{x}, \mathbf{x}^{\prime}, \mathbf{s}, \mathbf{s}^{\prime}, \mathbf{y}}\left(x, x^{\prime}, s, s^{\prime}, y\right) & =P_{\mathbf{x}, \mathbf{x}^{\prime}}\left(x, x^{\prime}\right) P_{\mathbf{s}, \mathbf{s}^{\prime} \mid \mathbf{x}, \mathbf{x}^{\prime}}\left(s, s^{\prime} \mid x, x^{\prime}\right) W_{\mathbf{y} \mid \mathbf{x}, \mathbf{s}}(y \mid x, s) \\
& =P_{\mathbf{x}, \mathbf{x}^{\prime}}\left(x, x^{\prime}\right) P_{\mathbf{s}, \mathbf{s}^{\prime} \mid \mathbf{x}, \mathbf{x}^{\prime}}\left(s, s^{\prime} \mid x, x^{\prime}\right) W_{\mathbf{y} \mid \mathbf{x}, \mathbf{s}}\left(y \mid x^{\prime}, s^{\prime}\right) .
\end{aligned}
$$

For any $\left(x, x^{\prime}\right) \in \mathcal{X}^{2}$, Equation (51) and Equation (52) are equal when marginalized out $\mathbf{s}, \mathbf{s}^{\prime}$, i.e.,

$$
\begin{aligned}
\sum_{s \in \mathcal{S}} P_{\mathbf{s} \mid \mathbf{x}, \mathbf{x}^{\prime}}\left(s \mid x, x^{\prime}\right) W_{\mathbf{y} \mid \mathbf{x}, \mathbf{s}}(y \mid x, s) & =\sum_{\left(s, s^{\prime}\right) \in \mathcal{S}^{2}} P_{\mathbf{s}, \mathbf{s}^{\prime} \mid \mathbf{x}, \mathbf{x}^{\prime}}\left(s, s^{\prime} \mid x, x^{\prime}\right) W_{\mathbf{y} \mid \mathbf{x}, \mathbf{s}}(y \mid x, s) \\
& =\sum_{\left(s, s^{\prime}\right) \in \mathcal{S}^{2}} P_{\mathbf{s}, \mathbf{s}^{\prime} \mid \mathbf{x}, \mathbf{x}^{\prime}}\left(s, s^{\prime} \mid x, x^{\prime}\right) W_{\mathbf{y} \mid \mathbf{x}, \mathbf{s}}\left(y \mid x, s^{\prime}\right)=\sum_{s^{\prime} \in \mathcal{S}} P_{\mathbf{s}^{\prime} \mid \mathbf{x}, \mathbf{x}^{\prime}}\left(s^{\prime} \mid x, x^{\prime}\right) W_{\mathbf{y} \mid \mathbf{y}, \mathbf{s}}\left(y \mid x^{\prime}, s^{\prime}\right) .
\end{aligned}
$$

It is worth noting that the above equation is precisely the one in Kiefer-Wolfowitz's notion of omniscient symmetrizability (Definition 24). Note also that Item 2 is equivalent to $\left[P_{\mathbf{x}, \mathbf{x}^{\prime}} P_{\mathbf{s}, \mathbf{s}^{\prime} \mid \mathbf{x}, \mathbf{x}^{\prime}}\right]_{\mathbf{s}}=\left[P_{\mathbf{x}, \mathbf{x}^{\prime}} P_{\mathbf{s} \mid \mathbf{x}, \mathbf{x}^{\prime}}\right]_{\mathbf{s}} \in \lambda_{\mathbf{s}}$ and $\left[P_{\mathbf{x}, \mathbf{x}^{\prime}} P_{\mathbf{s}, \mathbf{s}^{\prime} \mid \mathbf{x}, \mathbf{x}^{\prime}}\right]_{\mathbf{s}^{\prime}}=\left[P_{\mathbf{x}, \mathbf{x}^{\prime}} P_{\mathbf{s}^{\prime} \mid \mathbf{x}, \mathbf{x}^{\prime}}\right]_{\mathbf{s}^{\prime}} \in \lambda_{\mathbf{s}}$. Therefore, taking $U_{\mathbf{s} \mid \mathbf{x}, \mathbf{x}^{\prime}}:=\left[P_{\mathbf{s}, \mathbf{s}^{\prime} \mid \mathbf{x}, \mathbf{x}^{\prime}}\right]_{\mathbf{s}}$ and $U_{\mathbf{s}^{\prime} \mid \mathbf{x}, \mathbf{x}^{\prime}}:=\left[P_{\mathbf{s}, \mathbf{s}^{\prime} \mid \mathbf{x}, \mathbf{x}^{\prime}}\right]_{\mathbf{s}^{\prime}}$, we reduce Definition 27 to the following definition.

Definition 28 (Omniscient symmetrizability, two-distribution version). Given an omniscient AVC $\mathcal{A}_{\text {omni }}$, an input distribution $P_{\mathbf{x}} \in \lambda_{\mathbf{x}}$ is called omnisciently symmetrizable if for every $P_{\mathbf{x}, \mathbf{x}^{\prime}} \in \operatorname{CP}\left(P_{\mathbf{x}}\right)$, there are $U_{\mathbf{s} \mid \mathbf{x}, \mathbf{x}^{\prime}}, U_{\mathbf{s}^{\prime} \mid \mathbf{x}, \mathbf{x}^{\prime}} \in$ $\Delta\left(\mathcal{S} \mid \mathcal{X}^{2}\right)$ satisfying Equation (50) such that $\left[P_{\mathbf{x}, \mathbf{x}^{\prime}} U_{\mathbf{s} \mid \mathbf{x}, \mathbf{x}^{\prime}}\right]_{\mathbf{S}} \in \lambda_{\mathbf{s}}$ and $\left[P_{\mathbf{x}, \mathbf{x}^{\prime}} U_{\mathbf{s}^{\prime} \mid \mathbf{x}, \mathbf{x}^{\prime}}\right]_{\mathbf{s}^{\prime}} \in \lambda_{\mathbf{s}}$.

However, by the observation right after Definition 24, without loss of generality, we may as well restrict to a single symmetrizing distribution. The one-distribution version of Equation (50) is precisely the definition of $\mathcal{U}_{\text {omni,symm }}$ (Definition 24). We hence arrive at the following equivalent definition.

Definition 29 (Omniscient symmetrizability, one-distribution version). Given an omniscient AVC $\mathcal{A}_{\text {omni, }}$, an input distribution $P_{\mathbf{s}} \in \lambda_{\mathbf{x}}$ is called omnisciently symmetrizable if for every $P_{\mathbf{x}, \mathbf{x}^{\prime}} \in \operatorname{CP}\left(P_{\mathbf{x}}\right)$, there is a $U_{\mathbf{s} \mid \mathbf{x}, \mathbf{x}^{\prime}} \in \mathcal{U}_{\mathrm{omni}, \mathrm{symm}}$ such that $\left[P_{\mathbf{x}, \mathbf{x}^{\prime}} U_{\mathbf{s} \mid \mathbf{x}, \mathbf{x}^{\prime}}\right]_{\mathbf{s}} \in \lambda_{\mathbf{s}}$.

It is now easy to see that Definition 29 (which is an equivalent formulation of Definition 27) is a special case of Definition 21. Indeed, under the omniscient assumption $\mathbf{z}=\mathbf{x}$, the channel $W_{\mathbf{z} \mid \mathbf{x}}$ from Alice to James is noiseless, i.e., $W_{\mathbf{z} \mid \mathbf{x}}(z \mid x)=\mathbb{1}\{z=x\}$, and Definition 20 collapses to Definition 25 . 


\section{A seemingly more natural jamming strategy for capacity upper bound}

Given the definition of symmetrizing distributions (Definition 11), careful readers might expect it more natural to use distributions of the form $U_{\mathbf{s} \mid \mathbf{u}, \mathbf{x}_{1}, \cdots, \mathbf{x}_{L}}$ (rather than $U_{\mathbf{s} \mid \mathbf{u}}$ ) in Section XI. Specifically, they maybe would like to equip James with the following jamming strategy which seems more "compatible" with the definition of

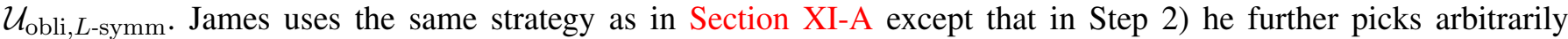
an $L$-list of codewords $\underline{\mathbf{x}}_{i_{1}}, \cdots, \underline{\mathbf{x}}_{i_{L}}\left(i_{1}<\cdots<i_{L}\right)$ whose type falls in the Voronoi cell of $\widetilde{P}_{\mathbf{x}_{1}, \cdots, \mathbf{x}_{L}}$. Then in

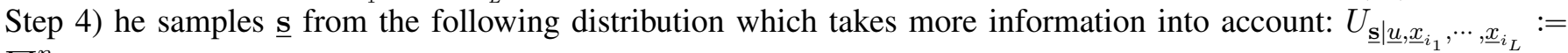
$\prod_{j=1}^{n} U_{\mathbf{s} \mid \mathbf{u}=\underline{u}(j), \mathbf{x}_{1}=\underline{x}_{i_{1}}(j), \cdots, \mathbf{x}_{L}=\underline{x}_{i_{L}}(j)}$.

We now argue that the above seemingly more judicious jamming strategy will never result in a stronger (i.e., smaller) upper bound on the $L$-list-decoding capacity. Hence the original jamming strategy we provided in Section XI-A is optimal. Via analysis (which we omit) similar to that in Section XI-B, the strategy described in the last paragraph gives the following capacity upper bound:

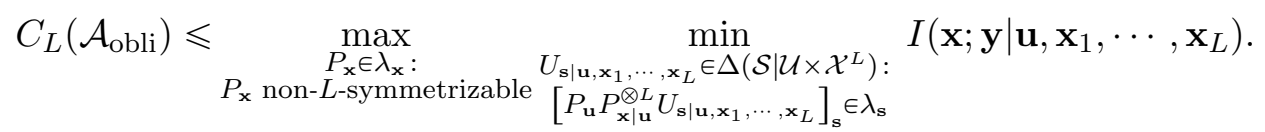

By elementary information inequalities, the above expression (Equation (53)) is no larger than Equation (5). Indeed,

$$
\begin{aligned}
I\left(\mathbf{x} ; \mathbf{y} \mid \mathbf{u}, \mathbf{x}_{1}, \cdots, \mathbf{x}_{L}\right) & =H\left(\mathbf{x} \mid \mathbf{u}, \mathbf{x}_{1}, \cdots, \mathbf{x}_{L}\right)-H\left(\mathbf{x} \mid \mathbf{u}, \mathbf{x}_{1}, \cdots, \mathbf{x}_{L}, \mathbf{y}\right) \\
& =H(\mathbf{x} \mid \mathbf{u})-H\left(\mathbf{x} \mid \mathbf{u}, \mathbf{x}_{1}, \cdots, \mathbf{x}_{L}, \mathbf{y}\right) \\
& =I\left(\mathbf{x} ; \mathbf{x}_{1}, \cdots, \mathbf{x}_{L}, \mathbf{y} \mid \mathbf{u}\right) \\
& =I(\mathbf{x} ; \mathbf{y} \mid \mathbf{u})+I\left(\mathbf{x} ; \mathbf{x}_{1}, \cdots, \mathbf{x}_{L} \mid \mathbf{u}, \mathbf{y}\right) \\
& \geqslant I(\mathbf{x} ; \mathbf{y} \mid \mathbf{u}) .
\end{aligned}
$$

Equation (54) is by the definition of mutual information (Definition 9). In Equation (55), we used the assumption $P_{\mathbf{x}, \mathbf{u}, \mathbf{x}_{1}, \cdots, \mathbf{x}_{L}}=P_{\mathbf{x}} P_{\mathbf{u}} P_{\mathbf{x} \mid \mathbf{u}}^{\otimes L}$ which implies that $\mathbf{x}$ and $\mathbf{x}_{1}, \cdots, \mathbf{x}_{L}$ are conditionally independent given $\mathbf{u}$. More specifically, as explained in the last section (Section XII-A), in the oblivious setting, we do not assume complete positivity of the joint distribution of the transmitted signal $\mathbf{x}$ and the spoofing list $\mathbf{x}_{1}, \cdots, \mathbf{x}_{L}$. In the definition of $L$-symmetrizability (Definition 14) and also the list-decoding capacity expression (Equation (5)), they are effectively independent. Equation (56) follows from chain rule for mutual information. Equation (57) is by nonnegativity of mutual information.

\section{XiII. Canonical Constructions of oblivious AVCs}

In this section, we introduce a machinery, which we call the canonical constructions of oblivious AVCs, for generating concrete oblivious channels with prescribed sets of $L$-symmetrizable input distributions. Besides being interesting in its own right, this will help us generate examples for which $L_{\mathrm{strong}}^{*}, L_{\mathrm{CP}}^{*}$ and $L_{\mathrm{weak}}^{*}$ are strictly different. To warm up, we first introduce canonical constructions of oblivious AVCs under unique-decoding in Section XIII-A. They will then be generalized to list-decoding in Section XIII-B. Finally, we use these constructions with carefully chosen components to separate different notions of list-symmetrizability due to [SG12] and us.

\section{A. Canonical constructions of oblivious AVCs}

Let $\mathcal{P} \subseteq \Delta(\mathcal{X})$ be a convex subset of distributions. Our goal is to construct an oblivious AVC $\mathcal{A}_{\text {obli }}=$ $\left(\mathcal{X}, \mathcal{S}, \mathcal{Y}, \lambda_{\mathbf{x}}, \lambda_{\mathbf{s}}, W_{\mathbf{y} \mid \mathbf{x}, \mathbf{s}}\right)$ whose symmetrizability set is precisely $\mathcal{P}$. Here the symmetrizability set $\mathcal{K}_{\text {obli }}\left(\mathcal{A}_{\text {obli }}\right)$ of $\mathcal{A}_{\mathrm{obli}}$ is defined as the set of (obliviously) symmetrizable input distributions:

$$
\mathcal{K}_{\text {obli }}\left(\mathcal{A}_{\text {obli }}\right):=\left\{P_{\mathbf{x}} \in \lambda_{\mathbf{x}}: P_{\mathbf{x}} \text { is symmetrizable }\right\} .
$$

Here we use Csiszár-Narayan's [CN88b] notion of symmetrizability (Definition 23).

The construction (which we refer to as the canonical construction) of $\mathcal{A}_{\text {obli,cano }}$ is as follows.

1) $\mathcal{X}$ is the same as the alphabet of $\mathcal{P}$.

2) $\mathcal{S}=\mathcal{X}$. 
3) $\mathcal{Y}=\left\{y_{x_{1}, x_{2}}\right\}_{\left(x_{1}, x_{2}\right) \in \mathcal{X}^{2}}$ where $\left(x_{1}, x_{2}\right)$ is an unordered $^{18}$ (possibly repeated) pair of input symbols. Note that $|\mathcal{Y}|=|\mathcal{X}|+\left(\begin{array}{c}|\mathcal{X}| \\ 2\end{array}\right)=\frac{1}{2}|\mathcal{X}|(|\mathcal{X}|+1)=\left(\begin{array}{c}|\mathcal{X}|+1 \\ 2\end{array}\right)$.

4) $\lambda_{\mathrm{x}}=\Delta(\mathcal{X})^{19}$.

5) $\lambda_{\mathbf{s}}=\mathcal{P}$.

6) $W_{\mathbf{y} \mid \mathbf{x}, \mathbf{s}}$ is a deterministic channel which we write as a (deterministic) function $W: \mathcal{X} \times \mathcal{S} \rightarrow \mathcal{Y}$ defined as $W(x, s)=y_{x, s}$.

We now compute $\mathcal{K}_{\text {obli }}\left(\mathcal{A}_{\text {obli,cano }}\right)$. To this end, we first compute $\mathcal{U}_{\text {obli,symm }}$. For $x=x^{\prime}$, the symmetrization identity in the definition of $\mathcal{U}_{\text {obli,symm }}$ (Definition 22) apparently holds. Fix $x \neq x^{\prime}$. The symmetrization identity gives us the following constraints on $U_{\mathbf{s} \mid \mathbf{x}}$.

1) If $y=y_{x, x}$ : LHS $=U_{\mathbf{s} \mid \mathbf{x}}\left(x \mid x^{\prime}\right)=0=$ RHS. Similarly the case where $y=y_{x^{\prime}, x^{\prime}}$ gives $0=U_{\mathbf{s} \mid \mathbf{x}}\left(x^{\prime} \mid x\right)$.

2) If $y=y_{x, s^{*}}$ for some $s^{*} \neq x, x^{\prime}$ : LHS $=U_{\mathbf{s} \mid \mathbf{x}}\left(s^{*} \mid x^{\prime}\right)=0=$ RHS. Similarly the case where $y=y_{x^{\prime}, s^{*}}$ for some $s^{*} \neq x, x^{\prime}$ gives $0=U_{\mathbf{s} \mid \mathbf{x}}\left(s^{*} \mid x\right)$.

3) If $y=y_{x, x^{\prime}}$ : LHS $=U_{\mathbf{s} \mid \mathbf{x}}\left(x^{\prime} \mid x^{\prime}\right)=U_{\mathbf{s} \mid \mathbf{x}}(x \mid x)=$ RHS.

Since the above conditions hold for all $x \neq x^{\prime}$, we have: if $U_{\mathbf{s} \mid \mathbf{x}}$ is written as an $|\mathcal{X}| \times|\mathcal{S}|$ matrix, then all offdiagonal entries are 0 and all diagonal entries are equal. Therefore, $U_{\mathbf{s} \mid \mathbf{x}}$ has to be an identity matrix (denoted by $\mathrm{id}_{\mathbf{s} \mid \mathbf{u}}$, defined as $\left.\operatorname{id}_{\mathbf{s} \mid \mathbf{x}}(s \mid x)=\mathbb{1}\{x=s\}\right)$ and $\mathcal{U}_{\text {obli,symm }}$ is a singleton set: $\mathcal{U}_{\text {obli,symm }}=\left\{\operatorname{id}_{\mathbf{s} \mid \mathbf{x}}\right\}$.

We now show $\mathcal{K}_{\text {obli }}\left(\mathcal{A}_{\text {obli,cano }}\right)=\mathcal{P}$.

The direction $\mathcal{K}_{\text {obli }}\left(\mathcal{A}_{\text {obli,cano }}\right) \supseteq \mathcal{P}$ is easy. Take any $P_{\mathbf{x}} \in \mathcal{P}$. We want to show that $P_{\mathbf{x}}$ is symmetrizable. Since $\mathcal{U}_{\text {obli,symm }}$ only contains the identity distribution, we just need to evaluate $\left[P_{\mathbf{x}} U_{\mathbf{s} \mid \mathbf{x}}\right]_{\mathbf{S}}=\left(\operatorname{diag}\left(P_{\mathbf{x}}\right) \mathbf{I}_{|\mathcal{X}| \times|\mathcal{S}|}\right)^{\top} \underline{\mathbb{1}}_{|\mathcal{S}|}=$ $P_{\mathbf{x}} \in \mathcal{P}=\lambda_{\mathbf{s}}$, where $\underline{\mathbb{1}}_{|\mathcal{S}|}$ denotes the all-one vector of length- $|\mathcal{S}|$. Therefore, $P_{\mathbf{s}}$ is symmetrized by $U_{\mathbf{s} \mid \mathbf{x}}=\mathbf{I}_{|\mathcal{X}| \times|\mathcal{S}|}$.

We then show the other direction $\mathcal{K}_{\text {obli }}\left(\mathcal{A}_{\text {obli,cano }}\right) \subseteq \mathcal{P}$ which is in fact similar. Take any symmetrizable input distribution $P_{\mathbf{x}}$. We want to show $P_{\mathbf{x}} \in \mathcal{P}$. Since $P_{\mathbf{x}}$ is symmetrizable and there is only one possible symmetrizing distribution, we have $\left[P_{\mathbf{x}} U_{\mathbf{s} \mid \mathbf{x}}\right]_{\mathbf{s}}=P_{\mathbf{x}} \in \lambda_{\mathbf{s}}=\mathcal{P}$.

Remark 12. Operationally, the identity symmetrizing distribution corresponds to the following jamming strategy. James simply uniformly samples a codeword $\underline{x}^{\prime}$ from the codebook and transmits it.

Remark 13. The above construction works for any subset $\mathcal{P}$ of input distributions, even for a non-convex subset. However, in this paper, we only focus on AVCs whose $\lambda_{\mathbf{s}}$ is convex to exclude exotic behaviours.

\section{B. Canonical construction of oblivious AVCs under list-decoding}

The above construction can be generalized to $L$-list-decoding in a natural way. For notational brevity, we take the $L=2$ case as an example. Given an arbitrary subset $\mathcal{P} \subseteq \Delta(\mathcal{X})$, the goal is to construct an oblivious AVC $\mathcal{A}_{\text {obli }}=\left(\mathcal{X}, \mathcal{S}, \mathcal{Y}, \lambda_{\mathbf{x}}, \lambda_{\mathbf{s}}, W_{\mathbf{y} \mid \mathbf{x}, \mathbf{s}}\right)$ whose 2-symmetrizability set is equal to $\mathcal{P}$. The 2-symmetrizability set of $\mathcal{A}$ is defined as:

$$
\mathcal{K}_{\mathrm{obli}, 2}\left(\mathcal{A}_{\mathrm{obli}}\right):=\left\{P_{\mathbf{x}} \in \lambda_{\mathbf{x}}: P_{\mathbf{x}} \text { is } 2 \text {-symmetrizable }\right\} .
$$

Recall that 2-symmetrizability is defined as follows, which is a special case of Definition 11.

Definition 30. The set of 2-symmetrizing distributions is defined as

$$
\mathcal{U}_{\text {obli,2-symm }}:=\left\{\begin{array}{cl} 
& \forall u \in \mathcal{U}, \forall\left(x_{0}, x_{1}, x_{2}, y\right) \in \mathcal{X}^{3} \times \mathcal{Y}, \forall \pi \in S_{3}, \\
U_{\mathbf{s} \mid \mathbf{u}, \mathbf{x}_{1}, \mathbf{x}_{2}} \in \Delta\left(\mathcal{S} \mid \mathcal{U} \times \mathcal{X}^{2}\right): & \sum_{s \in \mathcal{S}} U_{\mathbf{s} \mid \mathbf{u}=u, \mathbf{x}_{1}, \mathbf{x}_{2}}\left(s \mid x_{1}, x_{2}\right) W_{\mathbf{y} \mid \mathbf{x}, \mathbf{s}}\left(y \mid x_{0}, s\right) \\
= & \sum_{s \in \mathcal{S}} U_{\mathbf{s} \mid \mathbf{u}=u, \mathbf{x}_{1}, \mathbf{x}_{2}}\left(s \mid x_{\pi(1)}, x_{\pi(2)}\right) W_{\mathbf{y} \mid \mathbf{x}, \mathbf{s}}\left(y \mid x_{\pi(0)}, s\right)
\end{array}\right\} .
$$

Note that $\mathcal{U}_{\text {obli,1-symm }}=\mathcal{U}_{\text {obli,symm }}$.

The following definition is a special case of Definition 14. We list it below for the readers' convenience.

\footnotetext{
${ }^{18}$ That is, $y_{x_{1}, x_{2}}=y_{x_{2}, x_{1}}$

${ }^{19}$ In fact any $\lambda_{\mathbf{x}}$ satisfying $\mathcal{P} \subseteq \lambda_{\mathbf{x}} \subseteq \Delta(\mathcal{X})$ works.
} 
Definition 31. Given an oblivious AVC $\mathcal{A}_{\mathrm{obli}}$, an input distribution $P_{\mathbf{x}} \in \lambda_{\mathbf{x}}$ is called 2-symmetrizable if for every $P_{\mathbf{x}_{1}, \mathbf{x}_{2}} \in \mathrm{CP}\left(P_{\mathbf{x}}\right)$ and every CP-decomposition $\left(P_{\mathbf{u}}, P_{\mathbf{x} \mid \mathbf{u}}\right)$ of $P_{\mathbf{x}_{1}, \mathbf{x}_{2}}$, there is a $U_{\mathbf{s} \mid \mathbf{u}, \mathbf{x}_{1}, \mathbf{x}_{2}} \in \mathcal{U}_{\mathrm{obli}, 2 \text {-symm }}$ such that $\left[P_{\mathbf{u}} P_{\mathbf{x} \mid \mathbf{u}}^{\otimes 2} U_{\mathbf{s} \mid \mathbf{u}, \mathbf{x}_{1}, \mathbf{x}_{2}}\right]_{\mathbf{s}} \in \lambda_{\mathbf{s}}$

The canonical construction $\mathcal{A}_{\text {obli,2-cano }}$ is as follows.

1) $\mathcal{X}$ is the same as the alphabet of $\mathcal{P}$.

2) $\mathcal{S}=\mathcal{X}^{2}$.

3) $\mathcal{Y}=\left\{y_{x_{0}, x_{1}, x_{2}}\right\}_{\left(x_{0}, x_{1}, x_{2}\right) \in \mathcal{X}^{3}}$ where $\left(x_{0}, x_{1}, x_{2}\right)$ is an unordered ${ }^{20}$ (possibly not all distinct) triple of input symbols. Note that $|\mathcal{Y}|=|\mathcal{X}|+2\left(\begin{array}{c}|\mathcal{X}| \\ 2\end{array}\right)+\left(\begin{array}{c}|\mathcal{X}| \\ 3\end{array}\right)$.

4) $\lambda_{\mathrm{x}}=\Delta(\mathcal{X})^{21}$.

5) $\lambda_{\mathbf{s}}=\bigsqcup_{P_{\mathbf{x}} \in \mathcal{P}} \mathrm{CP}\left(P_{\mathbf{x}}\right)$. Note that $\lambda_{\mathbf{s}}$ is convex if $\mathcal{P}$ is convex. To see this, take a CP $P$-self-coupling $P_{\mathbf{x}_{1}, \mathbf{x}_{2}}=$ $\sum_{i=1}^{k} \lambda_{i} P_{i} P_{i}^{\top}$ and a CP $Q$-self-coupling $P_{\mathbf{x}_{1}, \mathbf{x}_{2}}^{\prime}=\sum_{j=1}^{\ell} \mu_{j} Q_{j} Q_{j}^{\top}$ for some $P, Q \in \mathcal{P}$, respectively. By selfcoupledness, $\left[P_{\mathbf{x}_{1}, \mathbf{x}_{2}}\right]_{\mathbf{x}_{1}}=\left[P_{\mathbf{x}_{1}, \mathbf{x}_{2}}\right]_{\mathbf{x}_{2}}=\sum_{i=1}^{k} \lambda_{i} P_{i}=P$ and $\left[P_{\mathbf{x}_{1}, \mathbf{x}_{2}}^{\prime}\right]_{\mathbf{x}_{1}}=\left[P_{\mathbf{x}_{1}, \mathbf{x}_{2}}^{\prime}\right]_{\mathbf{x}_{2}}=\sum_{j=1}^{\ell} \mu_{j} Q_{j}=Q$. We would like to show that for any $\alpha \in[0,1], R_{\mathbf{x}_{1}, \mathbf{x}_{2}}:=\alpha P_{\mathbf{x}_{1}, \mathbf{x}_{2}}+(1-\alpha) P_{\mathbf{x}_{1}, \mathbf{x}_{2}}^{\prime}$ is in $\lambda_{\mathbf{s}}$. First, $R_{\mathbf{x}_{1}, \mathbf{x}_{2}}=$ $\sum_{i=1}^{k} \alpha \lambda_{i} P_{i} P_{i}^{\top}+\sum_{j=1}^{\ell}(1-\alpha) \mu_{j} Q_{j} Q_{j}^{\top}$ is apparently CP. Second,

$$
\begin{aligned}
{\left[R_{\mathbf{x}_{1}, \mathbf{x}_{2}}\right]_{\mathbf{x}_{1}}\left(x_{1}\right) } & =\sum_{x_{2} \in \mathcal{X}}\left(\alpha \sum_{i=1}^{k} \lambda_{i} P_{i}\left(x_{1}\right) P_{i}\left(x_{2}\right)+(1-\alpha) \sum_{j=1}^{\ell} \mu_{j} Q_{j}\left(x_{1}\right) Q_{j}\left(x_{2}\right)\right) \\
& =\alpha \sum_{i=1}^{k} \lambda_{i} P_{i}\left(x_{1}\right)+(1-\alpha) \sum_{j=1}^{\ell} \mu_{j} Q_{j}\left(x_{1}\right) \\
& =\alpha P\left(x_{1}\right)+(1-\alpha) Q\left(x_{1}\right) .
\end{aligned}
$$

The above identity also holds for the $\mathbf{x}_{2}$ marginal. Therefore $R_{\mathbf{x}_{1}, \mathbf{x}_{2}}$ is an $(\alpha P+(1-\alpha) Q)$-self-coupling. By convexity of $\mathcal{P},(\alpha P+(1-\alpha) Q) \in \mathcal{P}$. Combined with complete positivity, we showed that $R_{\mathbf{x}_{1}, \mathbf{x}_{2}} \in \lambda_{\mathbf{s}}$.

6) $W_{\mathbf{y} \mid \mathbf{x}, \mathbf{s}}$ is a deterministic channel: $W\left(x_{0},\left(x_{1}, x_{2}\right)\right)=y_{x_{0}, x_{1}, x_{2}}$.

We claim that for any $\mathcal{U}$ (the alphabet of the time-sharing variable $\mathbf{u}$ ), $\mathcal{U}_{\mathrm{obli}, 2 \text {-symm }}=\left\{\mathrm{id}_{\mathbf{s} \mid \mathbf{u}, \mathbf{x}_{1}, \mathbf{x}_{2}}\right\}$ is a singleton set. Here $\operatorname{id}_{\mathbf{s} \mid \mathbf{u}, \mathbf{x}_{1}, \mathbf{x}_{2}}$ is defined according to: for any $u \in \mathcal{U}, \operatorname{id}_{\mathbf{s} \mid \mathbf{u}=u, \mathbf{x}_{1}, \mathbf{x}_{2}}\left(s \mid x_{1}, x_{2}\right)=\mathbb{1}\left\{s=\left(x_{1}, x_{2}\right)\right\}$. It is obvious that $\operatorname{id}_{\mathbf{s} \mid \mathbf{u}, \mathbf{x}_{1}, \mathbf{x}_{2}} \in \mathcal{U}_{\text {obli,2-symm }}$. We now show that any $U_{\mathbf{s} \mid \mathbf{u}, \mathbf{x}_{1}, \mathbf{x}_{2}}$ in $\mathcal{U}_{\text {obli,2-symm }}$ must be $\operatorname{id}_{\mathbf{s} \mid \mathbf{u}, \mathbf{x}_{1}, \mathbf{x}_{2}}$. Fix any $u \in \mathcal{U}$ and $\left(x_{1}, x_{2}\right) \in \mathcal{X}^{2}\left(x_{1}, x_{2}\right.$ can be the same). It suffices to show that $U_{\mathbf{s} \mid \mathbf{u}=u, \mathbf{x}_{1}, \mathbf{x}_{2}}\left(\left(x_{1}^{\prime}, x_{2}^{\prime}\right) \mid x_{1}, x_{2}\right)=0$ for all $\left(x_{1}^{\prime}, x_{2}^{\prime}\right) \in \mathcal{S}$ such that $\left\{x_{1}^{\prime}, x_{2}^{\prime}\right\} \neq\left\{x_{1}, x_{2}\right\}$. The condition $\left\{x_{1}^{\prime}, x_{2}^{\prime}\right\} \neq\left\{x_{1}, x_{2}\right\}$ implies $x_{1}^{\prime}, x_{2}^{\prime} \neq x_{1}$ or $x_{1}^{\prime}, x_{2}^{\prime} \neq x_{2}$. This is more easily seen by looking at the contrapositive:

$$
\begin{aligned}
& \left(\left\{x_{1}^{\prime}=x_{1} \text { or } x_{2}^{\prime}=x_{1}\right\} \cap\left\{x_{1}^{\prime}=x_{2} \text { or } x_{2}^{\prime}=x_{2}\right\}\right) \\
\Longrightarrow & \left(\left\{x_{1}^{\prime}=x_{1}, x_{2}^{\prime}=x_{2}\right\} \cup\left\{x_{1}^{\prime}=x_{2}, x_{2}^{\prime}=x_{1}\right\}\right) \equiv\left(\left\{x_{1}^{\prime}, x_{2}^{\prime}\right\}=\left\{x_{1}, x_{2}\right\}\right) .
\end{aligned}
$$

Assume $x_{1}^{\prime}, x_{2}^{\prime} \neq x_{1}$. The other case where $x_{1}^{\prime}, x_{2}^{\prime} \neq x_{2}$ is similar and we omit it. Consider $x_{0}, x_{1}, x_{2} \in$ $\mathcal{X}^{3}, y_{x_{0}, x_{1}^{\prime}, x_{2}^{\prime}} \in \mathcal{Y}$ and $\pi=\left(\begin{array}{lll}0 & 1 & 2 \\ 1 & 0 & 2\end{array}\right) \in S_{3}$. The symmetrization identity is specialized to:

$$
\text { LHS }=U_{\mathbf{s} \mid \mathbf{u}=u, \mathbf{x}_{1}, \mathbf{x}_{2}}\left(\left(x_{1}^{\prime}, x_{2}^{\prime}\right) \mid x_{1}, x_{2}\right)=0=\text { RHS. }
$$

This finishes the proof of the claim.

We now show that $\mathcal{K}_{\text {obli, } 2}(\mathcal{A})=\mathcal{P}$.

First, we show $\mathcal{K}_{\text {obli,2 }}\left(\mathcal{A}_{\text {obli,2-cano }}\right) \supseteq \mathcal{P}$. Take any $P_{\mathbf{x}} \in \mathcal{P}$. We claim that it is 2 -symmetrizable. Indeed, for any $P_{\mathbf{x}_{1}, \mathbf{x}_{2}} \in \mathrm{CP}\left(P_{\mathbf{x}}\right)$ and any CP-decomposition $\left(P_{\mathbf{u}}, P_{\mathbf{x} \mid \mathbf{u}}\right)$ of $P_{\mathbf{x}_{1}, \mathbf{x}_{2}},\left[P_{\mathbf{u}} P_{\mathbf{x} \mid \mathbf{u}}^{\otimes 2} \operatorname{id}_{\mathbf{s} \mid \mathbf{u}, \mathbf{x}_{1}, \mathbf{x}_{2}}\right]_{\mathbf{s}}=P_{\mathbf{x}_{1}, \mathbf{x}_{2}} \in \mathrm{CP}\left(P_{\mathbf{x}}\right) \subseteq \lambda_{\mathbf{s}}$. The equality is because: for any $s=\left(x_{1}^{*}, x_{2}^{*}\right) \in \mathcal{S}$,

$$
P_{\mathbf{S}}\left(\left(x_{1}^{*}, x_{2}^{*}\right)\right)=\sum_{\left(u, x_{1}, x_{2}\right) \in \mathcal{U} \times \mathcal{X}^{2}} P_{\mathbf{u}}(u) P_{\mathbf{x} \mid \mathbf{u}}\left(x_{1} \mid u\right) P_{\mathbf{x} \mid \mathbf{u}}\left(x_{2} \mid u\right) \operatorname{id}_{\mathbf{s} \mid \mathbf{u}, \mathbf{x}_{1}, \mathbf{x}_{2}}\left(s \mid u, x_{1}, x_{2}\right)
$$

${ }^{20}$ That is, $y_{x_{0}, x_{1}, x_{2}}=y_{x_{\pi(0)}, x_{\pi(1)}, x_{\pi(2)}}$ for all $\pi \in S_{3}$.

${ }^{21}$ Again, any $\lambda_{\mathbf{x}}$ such that $\mathcal{P} \subseteq \lambda_{\mathbf{x}} \subseteq \Delta(\mathcal{X})$ works. Indeed, We will use canonical constructions with a different $\lambda_{\mathbf{x}}$ to separate different notions of list-symmetrizability. 


$$
\begin{aligned}
& =\sum_{u \in \mathcal{U}} P_{\mathbf{u}}(u) P_{\mathbf{x} \mid \mathbf{u}}\left(x_{1}^{*} \mid u\right) P_{\mathbf{x} \mid \mathbf{u}}\left(x_{2}^{*} \mid u\right) \\
& =P_{\mathbf{x}_{1}, \mathbf{x}_{2}}\left(x_{1}^{*}, x_{2}^{*}\right) .
\end{aligned}
$$

Therefore, $P_{\mathbf{x}}$ is 2-symmetrized by $\operatorname{id}_{\mathbf{s} \mid \mathbf{u}, \mathbf{x}_{1}, \mathbf{x}_{2}} \in \mathcal{U}_{\text {obli,2-symm. }}$.

Second, we show $\mathcal{K}_{\text {obli,2 }}\left(\mathcal{A}_{\text {obli,2-cano }}\right) \subseteq \mathcal{P}$. Take any 2-symmetrizable input distribution $P_{\mathbf{x}}$. We want to show $P_{\mathbf{x}} \in \mathcal{P}$. By 2-symmetrizability, there is a CP extension $P_{\mathbf{x}_{1}, \mathbf{x}_{2}} \in \mathrm{CP}\left(P_{\mathbf{x}}\right)$ and a CP-decomposition $\left(P_{\mathbf{u}}, P_{\mathbf{x} \mid \mathbf{u}}\right)$ of $P_{\mathbf{x}_{1}, \mathbf{x}_{2}}$ such that $\left[P_{\mathbf{u}} P_{\mathbf{x} \mid \mathbf{u}}^{\otimes 2} \operatorname{id}_{\mathbf{s} \mid \mathbf{u}, \mathbf{x}_{1}, \mathbf{x}_{2}}\right]_{\mathbf{s}}=P_{\mathbf{x}_{1}, \mathbf{x}_{2}} \in \lambda_{\mathbf{s}}$. Since $P_{\mathbf{x}_{1}, \mathbf{x}_{2}} \in \mathrm{CP}\left(P_{\mathbf{x}}\right)$, by the definition of $\lambda_{\mathbf{s}}$ (Item 5), we have $P_{\mathbf{x}} \in \mathcal{P}$.

\section{Separating $L_{\mathrm{strong}}^{*}, L_{\mathrm{CP}}^{*}, L_{\text {weak }}^{*}$ via canonical constructions}

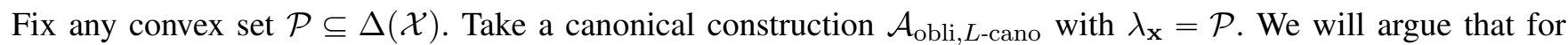
this example: $L_{\text {strong }}^{*}<L_{\mathrm{CP}}^{*}=L_{\text {weak }}^{*}=L$.

We claim that $L_{\mathrm{CP}}^{*}=L$. It is easy to check that $L_{\mathrm{CP}}^{*} \geqslant L$ since all input distributions $P_{\mathbf{x}} \in \lambda_{\mathbf{x}}=\mathcal{P}$ are CP- $L$ -

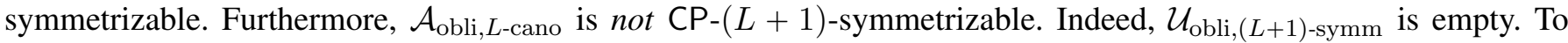
see this, one evaluates the symmetrization identity and can prove that for any $u \in \mathcal{U}$,

$$
U_{\mathbf{s} \mid \mathbf{u}=u, \mathbf{x}_{1}, \cdots, \mathbf{x}_{L}, \mathbf{x}_{L+1}}\left(\left(x_{1}^{\prime}, \cdots, x_{L}^{\prime}\right) \mid x_{0}, x_{1}, \cdots, x_{L}\right)=0
$$

for all $\left(x_{1}^{\prime}, \cdots, x_{L}^{\prime}\right) \in \mathcal{S}=\mathcal{X}^{L}$. We give a proof for the $L=2$ case and the general case is similar. Fix any $u \in \mathcal{U}$. In the $L=2$ case, we want to show $U_{\mathbf{s} \mid \mathbf{u}=u, \mathbf{x}_{1}, \mathbf{x}_{2}, \mathbf{x}_{3}}\left(\left(x_{1}^{\prime}, x_{2}^{\prime}\right) \mid x_{1}, x_{2}, x_{3}\right)=0$ for all $\left(x_{1}^{\prime}, x_{2}^{\prime}\right) \in \mathcal{S}=\mathcal{X}^{2}$ and $\left(x_{1}, x_{2}, x_{3}\right) \in \mathcal{X}^{3}$. For the symmetrization identity to be nontrivial, we had better take $x_{0}, x_{1}, x_{2}, x_{3}$ to be not all the same. Take $\left(x_{1}, x_{2}, x_{3}\right) \in \mathcal{X}^{3}$ and $x_{0} \in \mathcal{X} \backslash\left\{x_{1}, x_{2}, x_{3}\right\}$. Take $y_{x_{0}, x_{1}^{\prime}, x_{2}^{\prime}} \in \mathcal{Y}$ for any $\left(x_{1}^{\prime}, x_{2}^{\prime}\right) \in \mathcal{S}=\mathcal{X}^{2}$ and $\pi=\left(\begin{array}{llll}0 & 1 & 2 & 3 \\ 1 & 0 & 2 & 3\end{array}\right) \in S_{4}$. Note that at least one of the following cases hold: $x_{1} \notin\left\{x_{1}^{\prime}, x_{2}^{\prime}\right\}$ or $x_{2} \notin\left\{x_{1}^{\prime}, x_{2}^{\prime}\right\}$ or $x_{3} \notin\left\{x_{1}^{\prime}, x_{2}^{\prime}\right\}$. Assume WLOG $x_{1} \notin\left\{x_{1}^{\prime}, x_{2}^{\prime}\right\}$. Other two cases are similar. Since $x_{0} \neq x_{1}$, we have LHS $=$ $U_{\mathbf{s} \mid \mathbf{u}=u, \mathbf{x}_{1}, \mathbf{x}_{2}, \mathbf{x}_{3}}\left(\left(x_{1}^{\prime}, x_{2}^{\prime}\right) \mid x_{1}, x_{2}, x_{3}\right)=0=$ RHS. Therefore, $\mathcal{U}_{\text {obli,3-symm }}=\varnothing$.

We claim that $L_{\text {strong }}^{*}<L$. Apparently, by definition, $L_{\text {strong }}^{*} \leqslant L_{\text {CP }}^{*}$. It is left to show that $\mathcal{A}_{\text {obli, } L \text {-cano }}$ is not strongly- $L$-symmetrizable. In fact, no input distribution $P_{\mathbf{x}} \in \lambda_{\mathbf{x}}$ is strongly- $L$-symmetrizable. This is because: for any $P_{\mathbf{x}} \in \lambda_{\mathbf{x}}$ and any $P_{\mathbf{x}_{1}, \mathbf{x}_{2}} \in \mathcal{J}\left(P_{\mathbf{x}}\right) \backslash \mathrm{CP}\left(P_{\mathbf{x}}\right)^{22},\left[P_{\mathbf{x}_{1}, \mathbf{x}_{2}} \mathrm{id}_{\mathbf{s} \mid \mathbf{x}_{1}, \mathbf{x}_{2}}\right]_{\mathbf{s}}=P_{\mathbf{x}_{1}, \mathbf{x}_{2}} \notin \mathrm{CP}\left(P_{\mathbf{x}}\right)$ and hence $\left[P_{\mathbf{x}_{1}, \mathbf{x}_{2}} \mathrm{id}_{\mathbf{s}} \mid \mathbf{x}_{1}, \mathbf{x}_{2}\right]_{\mathbf{s}} \notin \lambda_{\mathbf{s}}=\bigsqcup_{P_{\mathbf{x}} \in \mathcal{P}} \mathrm{CP}\left(P_{\mathbf{x}}\right)$.

We claim that $L_{\text {weak }}^{*}=L$. By definition, $L_{\text {weak }}^{*} \geqslant L_{\mathrm{CP}}^{*}=L$. Furthermore, since $\mathcal{U}_{\mathrm{obli},(L+1) \text {-symm }}=\varnothing, L_{\text {weak }}^{*}<$ $L+1$. This proves the claim.

We now give an example for which $L_{\text {CP }}^{*}<L_{\text {weak }}^{*}=L$. Let $\mathcal{P}=\left\{P_{\mathbf{x}}\right\}$ be a singleton set for some $P_{\mathbf{x}} \in \Delta(\mathcal{X})$.

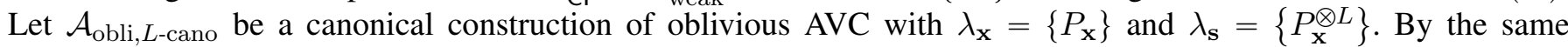
argument as before, $L_{\text {weak }}^{*}=L$. However, for non-product CP distributions $P_{\mathbf{x}_{1}, \cdots, \mathbf{x}_{L}} \in \mathrm{CP}^{\otimes L}\left(P_{\mathbf{x}}\right) \backslash\left\{P_{\mathbf{x}}^{\otimes L}\right\}^{23}$, no matter which CP-decomposition we take, $\left[P_{\mathbf{u}, \mathbf{x}_{1}, \cdots, \mathbf{x}_{L}} \operatorname{id}_{\mathbf{s} \mid \mathbf{u}, \mathbf{x}_{1}, \cdots, \mathbf{x}_{L}}\right]_{\mathbf{s}}=P_{\mathbf{x}_{1}, \cdots, \mathbf{x}_{L}} \notin\left\{P_{\mathbf{x}}^{\otimes L}\right\}=\lambda_{\mathbf{s}}$. Therefore $L_{\mathrm{CP}}^{*}<L$.

\section{CONCLUDING REMARKS AND OPEN PROBLEMS}

In this paper, we revisited the classical problem of list-decoding for oblivious Arbitrarily Varying Channels. We pinned down the exact threshold of list-size that determines the positivity of list-decoding capacity. The proof utilized a machinery recently developed in [WBBJ19] and [ZBJ20]. A natural capacity lower bound was proved. However, our capacity upper bound is conditioned on Conjecture 21.

We list several open problems for future research.

1) The most obvious open question is to obtain a tight characterization of the list-decoding capacity. This amounts to proving/disproving/bypassing Conjecture 21. We do believe that Conjecture 21 is true and our lower bound (Equation (5)) is tight, though proving it requires new ideas. Otherwise, one could try to develop other jamming strategies whose analysis bypasses this conjecture.

\footnotetext{
${ }^{22}$ Note that $\mathcal{J}\left(P_{\mathbf{x}}\right) \backslash \mathrm{CP}\left(P_{\mathbf{x}}\right) \neq \varnothing$.

${ }^{23}$ Note that $\mathrm{CP}^{\otimes L}\left(P_{\mathbf{x}}\right) \backslash\left\{P_{\mathbf{x}}^{\otimes L}\right\} \neq \varnothing$.
} 
2) In our capacity bounds (Equation (5)), the maximization (implicitly) includes searching over all CP-decompositions $\left(P_{\mathbf{u}}, P_{\mathbf{x} \mid \mathbf{u}}\right)$ of a CP-distribution $P_{\mathbf{x}_{[L]}} \in \mathrm{CP}^{\otimes L}\left(P_{\mathbf{x}}\right)$. As mentioned in Remark 7, a CP-distribution can have multiple CP-decompositions. Among all decompositions, the smallest induced $|\mathcal{U}|$ is called the CP-rank. We do not know whether decompositions with $|\mathcal{U}|>C P-r k\left(P_{\mathbf{x}_{[L]}}\right)$ will ever be maximizers. Also, there are $\mathrm{CP}$-distributions whose $\mathrm{CP}$-decompositions can have arbitrarily large $|\mathcal{U}|$. For the purpose of maximizing the mutual information in Equation (5), we do not have a cardinality bound on $\mathcal{U}$ of the CP-decomposition. Therefore, Equation (5) is not computable for general AVCs. On the other hand, it may be the case that for some channels, to approach the maximum of Equation (5), we cannot put an upper bound on $|\mathcal{U}|$. We leave this issue for future exploration.

3) We only found (via the canonical constructions) examples of oblivious AVCs for which $L_{\text {strong }}^{*}<L_{\mathrm{CP}}^{*}=L_{\mathrm{weak}}^{*}$ and other examples of oblivious AVCs for which $L_{\mathrm{CP}}^{*}<L_{\text {weak }}^{*}$. We are curious to see a single example for which $L_{\text {strong }}^{*}<L_{\mathrm{CP}}^{*}<L_{\text {weak }}^{*}$.

4) It is possible to extend our results to multiuser channels. Arguably the most well-understood multiuser channels would be the Multiple Access Channels (MACs). A two-user MAC consists of two transmitters who transmit their encodings simultaneously and a single receiver who wants to decode both messages. In the presence of an oblivious adversary, the fundamental limits of MACs are not fully understood until recently [Jah81], [AC99], [PS19b]. For the list-decoding variant, Cai [Cai16] gave the right notion of list-symmetrizability for MACs and completely determined the list-decoding capacity of unconstrained MACs. To complete the picture along this line of research, it is desirable to extend Cai's results to the constrained case. If one follows the techniques in this paper, this will likely require us to first prove a Plotkin-type converse for omniscient MACs, which itself is highly nontrivial and intriguing. Given the obstacles even in the point-to-point case, a complete characterization of the list-decoding capacity of MACs seems challenging.

5) Guruswami and Smith [GS16] constructed explicit codes equipped with (stochastic) encoders and decoders that run in polynomial time for the oblivious bitflip channels. Towards explicit fast encodable/decodable code constructions for general oblivious AVCs, it is worth exploring the limits to Guruswami-Smith's techniques.

\section{REFERENCES}

[AC99] Rudolf Ahlswede and Ning Cai. Arbitrarily varying multiple-access channels. i. ericson's symmetrizability is adequate, gubner's conjecture is true. IEEE Transactions on Information Theory, 45(2):742-749, 1999. II-D, 4

[AC00] Rudolf Ahlswede and Ning Cai. The avc with noiseless feedback and maximal error probability: a capacity formula with a trichotomy. In Numbers, Information and Complexity, pages 151-176. Springer, 2000. II-D

[Ahl78] R. Ahlswede. Elimination of correlation in random codes for arbitrarily varying channels. Z. Wahrscheinlichkeitstheorie Verv. Gebiete, 44:181-193, 1978. II-A, II-D

[BBT60] David Blackwell, Leo Breiman, and AJ Thomasian. The capacities of certain channel classes under random coding. The Annals of Mathematical Statistics, 31(3):558-567, 1960. I

[BD20] Marco Bondaschi and Marco Dalai. Revisiting zero-rate bounds on the reliability function of discrete memoryless channels. In 2020 IEEE International Symposium on Information Theory (ISIT). IEEE, 2020. A-0a

$\left[\mathrm{BDJ}^{+} 20\right]$ Amitalok J. Budkuley, Bikash Dey, Sidharth Jaggi, Michael Langberg, Anand Sarwate, and Carol Wang. Symmetrizability for Myopic AVCs. In 2020 IEEE International Symposium on Information Theory (ISIT). IEEE, 2020. II-D, XII-A, 21, XII-A, 24

[Ber64] Elwyn R Berlekamp. Block coding with noiseless feedback. PhD thesis, Massachusetts Institute of Technology, 1964. II-D

[BK20] Ainesh Bakshi and Pravesh Kothari. List-decodable subspace recovery via sum-of-squares. arXiv preprint arXiv:2002.05139, 2020. I

[BL09] Vladimir Blinovsky and Simon Litsyn. New asymptotic bounds on the size of list codes on euclidean sphere. In 2009 IEEE International Symposium on Information Theory, pages 1244-1247. IEEE, 2009. II-B

[Bla62] N. Blachman. On the capacity of bandlimited channel perturbed by statistically dependent interference. IRE Transactions on Information Theory, 8:48-55, 1962. II-B

[Bli86] Vladimir M Blinovsky. Bounds for codes in the case of list decoding of finite volume. Problems of Information Transmission, 22:7-19, 1986. II-B

[Bli97] Volodia Blinovsky. Multiple packing of euclidean sphere. In Proceedings of IEEE International Symposium on Information Theory, page 18. IEEE, 1997. II-B

[Bli05] Vladimir M Blinovsky. Code bounds for multiple packings over a nonbinary finite alphabet. Problems of Information Transmission, 41:23-32, 2005. II-B

[Bli08] Vladimir M Blinovsky. On the convexity of one coding-theory function. Problems of Information Transmission, 44:34-39, 2008. II-B

[Cai16] Ning Cai. List decoding for arbitrarily varying multiple access channel revisited: List configuration and symmetrizability. IEEE Transactions on Information Theory, 62(11):6095-6110, 2016. 4

[CJL15] Zitan Chen, Sidharth Jaggi, and Michael Langberg. A characterization of the capacity of online (causal) binary channels. In Proceedings of the forty-seventh annual ACM symposium on Theory of computing, pages 287-296, 2015. II-D 
[CK81] I. Csiszár and J. Körner. On the capacity of the arbitrarily varying channel for maximum probability of error. Z Wahrscheinlichkeitstheorie Verv. Gebiete, 57:87-101, 1981. II-B

[CMY20] Yeshwanth Cherapanamjeri, Sidhanth Mohanty, and Morris Yau. List decodable mean estimation in nearly linear time. arXiv preprint arXiv:2005.09796, 2020. I

[CN88a] Imre Csiszár and Prakash Narayan. Arbitrarily varying channels with constrained inputs and states. IEEE Trans. Inf. Theory, 34:27-34, 1988. II-D

[CN88b] Imre Csiszár and Prakash Narayan. The Capacity of the Arbitrarily Varying Channel Revisited : Positivity, Constraints. IEEE Trans. Inf. Theory, 34:181-193, 1988. II-A, II-B, 2, 4, III, 9, IX, X, XII-A, XII-A, 23, XII-A, 25, XII-A, XIII-A, C

[CN91] I. Csiszár and P. Narayan. Capacity of the Gaussian Arbitrarily Varying Channel. IEEE Trans. Inf. Theory, 37:18-26, 1991. II-A

[CT91] T.M. Cover and J.A. Thomas. Elements of Information Theory. Wiley, New York, 1991. VI-D

[DC83] Dominique De Caen. Extension of a theorem of Moon and Moser on complete subgraphs. Faculty of Mathematics, University of Waterloo, 1983. 11

$\left[\mathrm{DJL}^{+}\right.$19a] B. Kumar Dey, S. Jaggi, M. Langberg, A. D. Sarwate, and C. Wang. The interplay of causality and myopia in adversarial channel models. In 2019 IEEE International Symposium on Information Theory (ISIT), pages 1002-1006, 2019. II-D

[DJL19b] Bikash Kumar Dey, Sidharth Jaggi, and Michael Langberg. Sufficiently myopic adversaries are blind. IEEE Transactions on Information Theory, 65(9):5718-5736, 2019. II-D

[DJLS13] B.K. Dey, S. Jaggi, M. Langberg, and A.D. Sarwate. Upper Bounds on the Capacity of Binary Channels With Causal Adversaries. IEEE Trans. Inf. Theory, 59:3753-3763, June 2013. II-D

[DJLS16] B. K. Dey, S. Jaggi, M. Langberg, and A. D. Sarwate. The benefit of a 1-bit jump-start, and the necessity of stochastic encoding, in jamming channels. Technical Report arXiv:1602.02384 [cs.IT], ArXiV, February 2016. II-D

[DKK20] Ilias Diakonikolas, Daniel M Kane, and Daniel Kongsgaard. List-decodable mean estimation via iterative multi-fitering. arXiv preprint arXiv:2006.10715, 2020. I

[DKS18] Ilias Diakonikolas, Daniel M Kane, and Alistair Stewart. List-decodable robust mean estimation and learning mixtures of spherical gaussians. In Proceedings of the 50th Annual ACM SIGACT Symposium on Theory of Computing, pages 1047-1060, 2018. I

[DMOZ19] Dean Doron, Dana Moshkovitz, Justin Oh, and David Zuckerman. Nearly optimal pseudorandomness from hardness. In Electronic Colloquium on Computational Complexity (ECCC), volume 26, page 99, 2019. I

[EGK11] Abbas El Gamal and Young-Han Kim. Network Information Theory. Cambridge University Press, 2011. 22

[Eli57] Peter Elias. List decoding for noisy channels. 1957. I

[Eri85] T Ericson. Exponential error bounds for random codes in the arbitrarily varying channel. IEEE Transactions on Information Theory, 31(1):42-48, 1985. II-A

[Gil52] Edgar N Gilbert. A comparison of signalling alphabets. The Bell system technical journal, 31(3):504-522, 1952. II-B

[GS16] Venkatesan Guruswami and Adam Smith. Optimal rate code constructions for computationally simple channels. Journal of the ACM (JACM), 63(4):1-37, 2016. 5

[Gur04] V. Guruswami. List Decoding of Error Correcting Codes (Lecture Notes in Computer Science),. Springer-Verlag, NY, 2004. I [Gur07] Venkatesan Guruswami. Algorithmic results in list decoding. Now Publishers Inc, 2007. I

[HK16] Fatemeh Hosseinigoki and Oliver Kosut. The gaussian interference channel in the presence of a malicious jammer. In 2016 54th Annual Allerton Conference on Communication, Control, and Computing (Allerton), pages 679-686. IEEE, 2016. II-D

[HK18] Fatemeh Hosseinigoki and Oliver Kosut. Capacity of the gaussian arbitrarily-varying channel with list decoding. IEEE International Symposium on Information Theory, 2018. II-A

[HK19] Fatemeh Hosseinigoki and Oliver Kosut. Capacity of gaussian arbitrarily-varying fading channels. In 2019 53rd Annual Conference on Information Sciences and Systems (CISS), pages 1-6. IEEE, 2019. II-D

[HK20] Fatemeh Hosseinigoki and Oliver Kosut. Capacity region of the gaussian arbitrarily-varying broadcast channel. In 2020 IEEE International Symposium on Information Theory (ISIT), pages 1007-1011. IEEE, 2020. II-D

[HKV15] Bernhard Haeupler, Pritish Kamath, and Ameya Velingker. Communication with partial noiseless feedback. In Approximation, Randomization, and Combinatorial Optimization. Algorithms and Techniques (APPROX/RANDOM 2015). Schloss DagstuhlLeibniz-Zentrum fuer Informatik, 2015. II-D

[Hug97] Brian L. Hughes. The smallest list for the arbitrarily varying channel. IEEE Transactions on Information Theory, 43(3):803-815, 1997. II-A, 2, III, III, III, 9, IX, X, 33, D

[Jah81] J-H Jahn. Coding of arbitrarily varying multiuser channels. IEEE Transactions on Information Theory, 27(2):212-226, 1981. II-D, 4

[JL17] Sidharth Jaggi and Michael Langberg. Two-way interference channels with jammers. In Proc. IEEE Int. Symp. Information Theory, 2017. II-D

[Kee11] Peter Keevash. Hypergraph turan problems. Surveys in combinatorics, 392:83-140, 2011.11

[KKK19] Sushrut Karmalkar, Adam Klivans, and Pravesh Kothari. List-decodable linear regression. In Advances in Neural Information Processing Systems, pages 7425-7434, 2019. I

[KL78] Grigorii Anatolevich Kabatiansky and Vladimir Iosifovich Levenshtein. On bounds for packings on a sphere and in space. Problemy Peredachi Informatsii, 14(1):3-25, 1978. II-B

[KO98] Janos Korner and Alon Orlitsky. Zero-error information theory. IEEE Transactions on Information Theory, 44(6):2207-2229, 1998. II-C

[Kom90] János Komlós. A strange pigeon-hole principle. Order, 7(2):107-113, 1990. A-0a

[KW62] J. Kiefer and Jacob Wolfowitz. Channels with arbitrarily varying channel probability functions. Information and Control, 5(1):44-54, 1962. II-B, XII-B, 24, 26, XII-B 
$\left[\mathrm{LDJ}^{+}\right.$18] Tongxin Li, Bikash Kumar Dey, Sidharth Jaggi, Michael Langberg, and Anand D Sarwate. Quadratically constrained channels with causal adversaries. In 2018 IEEE International Symposium on Information Theory (ISIT), pages 621-625. IEEE, 2018. II-D

[LN98] A. Lapidoth and P. Narayan. Reliable Communication under Channel Uncertainty. IEEE Trans. Inf. Theory, 44:2148-2177, 1998. I

[Lov79] László Lovász. On the shannon capacity of a graph. IEEE Transactions on Information theory, 25(1):1-7, 1979. II-C

[MRRW77] R. J. McEliece, E. R. Rodemich, H. Jr. Rumsey, and L. R. Welch. New upper bounds on the rate of a code via the DelsarteMacWilliams inequalities. IEEE Trans. Inf. Theory, 23, 1977. II-B

[Pol16] Yury Polyanskiy. Upper bound on list-decoding radius of binary codes. IEEE Transactions on Information Theory, 62(3):11191128, 2016. II-B

[PS17] Uzi Pereg and Yossef Steinberg. The arbitrarily varying broadcast channel with degraded message sets with causal side information at the encoder. arXiv preprint arXiv:1709.04770, 2017. II-D

[PS18] Uzi Pereg and Yossef Steinberg. The arbitrarily varying gaussian relay channel with sender frequency division. In 2018 56th Annual Allerton Conference on Communication, Control, and Computing (Allerton), pages 1097-1103. IEEE, 2018. II-D

[PS19a] Uzi Pereg and Yossef Steinberg. The arbitrarily varying relay channel. Entropy, 21(5):516, 2019. II-D

[PS19b] Uzi Pereg and Yossef Steinberg. The capacity region of the arbitrarily varying mac: with and without constraints. In 2019 IEEE International Symposium on Information Theory (ISIT), pages 445-449. IEEE, 2019. II-D, 4

[RY20a] Prasad Raghavendra and Morris Yau. List decodable learning via sum of squares. In Proceedings of the Fourteenth Annual ACM-SIAM Symposium on Discrete Algorithms, pages 161-180. SIAM, 2020. I

[RY20b] Prasad Raghavendra and Morris Yau. List decodable subspace recovery. arXiv preprint arXiv:2002.03004, 2020. I

[SG12] Anand D. Sarwate and Michael Gastpar. List-decoding for the arbitrarily varying channel under state constraints. IEEE Transactions on Information Theory, 58(3):1372-1384, 2012. II-A, 2, III, III, IV, 9, VII, VIII, VIII-A, VIII-A, 4, VIII-A, 1, 2, 4, 5, 5, IX, X, XIII, 28, 14

[Sha56] Claude Shannon. The zero error capacity of a noisy channel. IRE Transactions on Information Theory, 2(3):8-19, 1956. II-C

[Var57] Rom Rubenovich Varshamov. Estimate of the number of signals in error correcting codes. Docklady Akad. Nauk, SSSR, 117:739-741, 1957. II-B

[WBBJ19] Xishi Wang, Amitalok J. Budkuley, Andrej Bogdanov, and Sidharth Jaggi. When are large codes possible for AVCs? In 2019 IEEE International Symposium on Information Theory (ISIT), pages 632-636. IEEE, 2019. II-B, III, XII-A, XII-B, 27, XII-B, 27, XIV

[Woz58] John M Wozencraft. List decoding. Quarterly Progress Report, 48:90-95, 1958. I

[ZBJ20] Yihan Zhang, Amitalok J. Budkuley, and Sidharth Jaggi. Generalized List Decoding. In Thomas Vidick, editor, 11th Innovations in Theoretical Computer Science Conference (ITCS 2020), volume 151 of Leibniz International Proceedings in Informatics (LIPIcs), pages 51:1-51:83, Dagstuhl, Germany, 2020. Schloss Dagstuhl-Leibniz-Zentrum fuer Informatik. II-B, III, 1, 1, VI-C, 16, IX, XIV, A, A-0b

[Zig76] Kamil'Shamil'evich Zigangirov. On the number of correctable errors for transmission over a binary symmetrical channel with feedback. Problemy Peredachi Informatsii, 12(2):3-19, 1976. II-D

[ZVJ20] Yihan Zhang, Shashank Vatedka, and Sidharth Jaggi. Quadratically constrained two-way adversarial channels. arXiv preprint arXiv:2001.02575, 2020. II-D

[ZVJS18] Yihan Zhang, Shashank Vatedka, Sidharth Jaggi, and Anand D Sarwate. Quadratically constrained myopic adversarial channels. In 2018 IEEE International Symposium on Information Theory (ISIT), pages 611-615. IEEE, 2018. II-D

\section{APPENDIX A}

\section{ROBUST GENERALIZED PLOTKIN BOUND}

In this section, we give a proof sketch of Theorem 16. It is essentially a corollary of [ZBJ20] with a twist that the code can be approximately $\widehat{P}_{\mathbf{x}}$-constant-composition rather than exactly $\widehat{P}_{\mathbf{x}}$-constant-composition. We prove that the generalized Plotkin bound in [ZBJ20] still holds for approximate constant-composition codes. The proof is basically the same with more slack factors to take care of.

We will give a proof sketch to a stronger theorem. To state the stronger theorem, we need several definitions.

Definition 32 (Approximate self-coupling). Let $\lambda>0$ be a constant and $\widehat{P}_{\mathbf{x}} \in \Delta(\mathcal{X})$. A joint distribution $P_{\mathbf{x}_{1}, \cdots, \mathbf{x}_{L}} \in$ $\Delta\left(\mathcal{X}^{L}\right)$ is called a $\left(\lambda, \widehat{P}_{\mathbf{x}}\right)$-self-coupling if

$$
d\left(\left[P_{\mathbf{x}_{1}, \cdots, \mathbf{x}_{L}}\right]_{\mathbf{x}_{i}}, \widehat{P}_{\mathbf{x}}\right) \leqslant \lambda
$$

for every $1 \leqslant i \leqslant L$. When $\lambda=0$, we say that $P_{\mathbf{x}_{1}, \cdots, \mathbf{x}_{L}}$ is a $\widehat{P}_{\mathbf{x}}$-self-coupling which agrees with Definition 12 . The set of $\left(\lambda, \widehat{P}_{\mathbf{x}}\right)$-self-couplings of order- $L$ is denoted by $\mathcal{J}_{\lambda}^{\otimes L}\left(\widehat{P}_{\mathbf{x}}\right)$. When $\lambda=0, \mathcal{J}_{0}^{\otimes L}\left(\widehat{P}_{\mathbf{x}}\right)=\mathcal{J}^{\otimes L}\left(\widehat{P}_{\mathbf{x}}\right)$.

For every approximate self-coupling, there is always an exact self-coupling close by.

Lemma 28 (Distribution approximation [SG12]). Suppose a joint distribution $\widehat{P}_{\mathbf{x}_{1}, \cdots, \mathbf{x}_{L}}$ is a $\left(\lambda, \widehat{P}_{\mathbf{x}}\right)$-self-coupling for some $\widehat{P}_{\mathbf{x}} \in \Delta(\mathcal{X})$. Then there exists a $\widehat{P}_{\mathbf{x}}$-self-coupling $\widetilde{P}_{\mathbf{x}_{1}, \cdots, \mathbf{x}_{L}}$ such that $d\left(\widetilde{P}_{\mathbf{x}_{1}, \cdots, \mathbf{x}_{L}}, \widehat{P}_{\mathbf{x}_{1}, \cdots, \mathbf{x}_{L}}\right) \leqslant f(\lambda)$ for some $f(\lambda)>0$ such that $f(\lambda) \stackrel{\lambda \rightarrow 0}{\longrightarrow} 0$. 
Remark 14. In the original paper [SG12], Sarwate and Gastpar chose to use the $\ell^{\infty}$-norm, i.e., $d_{\ell^{\infty}}(P, Q):=$ $\max _{x \in \mathcal{X}}|P(x)-Q(x)|$ for $P, Q \in \Delta(\mathcal{X})$. Despite their choice, $\ell^{1}$-norm (which we use in this paper) only differs from $\ell^{\infty}$-norm by a multiplicative constant factor. Indeed, for any constant-sized alphabet $\mathcal{X}$ and any constant list-size $L \in \mathbb{Z}_{\geqslant 1}$, for any $P_{\mathbf{x}_{[L]}}, Q_{\mathbf{x}_{[L]}} \in \Delta\left(\mathcal{X}^{L}\right), d_{\ell^{\infty}}\left(P_{\mathbf{x}_{[L]}}, Q_{\mathbf{x}_{[L]}}\right) \leqslant d_{\ell^{1}}\left(P_{\mathbf{x}_{[L]}}, Q_{\mathbf{x}_{[L]}}\right) \leqslant|\mathcal{X}|^{L} d_{\ell^{\infty}}\left(P_{\mathbf{x}_{[L]}}, Q_{\mathbf{x}_{[L]}}\right)$. Therefore, up to multiplicative constant factors, Lemma 28 holds under $d_{\ell^{1}}$ as well.

Definition 33 (Robust confusability set). Let $\mathcal{A}_{\text {omni }}=\left(\mathcal{X}, \mathcal{S}, \mathcal{Y}, \lambda_{\mathbf{x}}, \lambda_{\mathbf{s}}, W_{\mathbf{y} \mid \mathbf{x}, \mathbf{s}}\right)$ be an omniscient channel with a 0-1 channel transition distribution $W_{\mathbf{y} \mid \mathbf{x}, \mathbf{s}}$ and convex input \& state constraints $\lambda_{\mathbf{x}} \subseteq \Delta(\mathcal{X})$ and $\lambda_{\mathbf{s}} \subseteq \Delta(\mathcal{S})$, respectively. Let $\lambda>0$ be a constant and $P_{\mathbf{x}} \in \lambda_{\mathbf{x}}$. Define the robust confusability set associated to this channel as

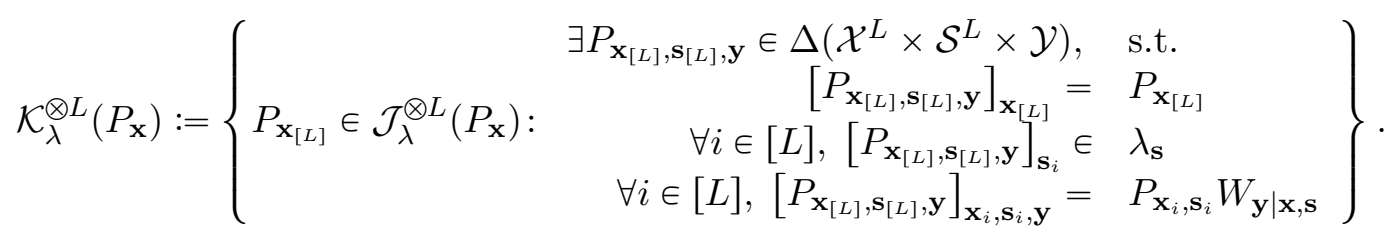

When $\lambda=0$, we write $\mathcal{K}^{\otimes L}\left(P_{\mathbf{x}}\right)$ for $\mathcal{K}_{\lambda}^{\otimes L}\left(P_{\mathbf{x}}\right)$.

Theorem 29 (Robust generalized Plotkin bound, general form). Assume that

$$
d\left(\mathcal{J}^{\otimes L}\left(P_{\mathbf{x}}\right) \backslash \mathcal{K}^{\otimes L}\left(P_{\mathbf{x}}\right), \mathrm{CP}^{\otimes L}\left(P_{\mathbf{x}}\right)\right) \geqslant \varepsilon
$$

for some constant $\varepsilon>0$. Let $\mathcal{C}$ be a $\left(\lambda, P_{\mathbf{x}}\right)$-constant-composition code satisfying $\Gamma^{\otimes L}(\mathcal{C}) \subset \mathcal{K}_{\lambda}^{\otimes L}\left(P_{\mathbf{x}}\right)$. Then there is a constant $K=K(\varepsilon, \lambda, L,|\mathcal{X}|)>0$ such that $|\mathcal{C}| \leqslant K$.

Remark 15. Note that $K$ does not depend on $n$ - the blocklength of $\mathcal{C}$.

Remark 16. Apparently, the condition given by Equation (58) implies the condition given by Equation (9) in Theorem 16. Hence Theorem 29 implies Theorem 16.

Proof sketch of Theorem 29.. Take an $\eta$-net $\mathcal{N}$ of $\mathcal{J}_{\lambda}^{\otimes L}\left(P_{\mathbf{x}}\right) \backslash \mathcal{K}_{\lambda}^{\otimes L}\left(P_{\mathbf{x}}\right)$. Note that $|\mathcal{N}|$ is a constant. Use hypergraph Ramsey's theorem to find a subcode $\mathcal{C}^{\prime} \subset \mathcal{C}$ such that $d\left(\Gamma^{\otimes L}\left(\mathcal{C}^{\prime}\right), P_{\mathbf{x}_{[L]}}^{\prime}\right) \leqslant \eta$ for some $P_{\mathbf{x}_{[L]}}^{\prime} \in \mathcal{N}$. The subcode is guaranteed to satisfy $\left|\mathcal{C}^{\prime}\right| \stackrel{|\mathcal{C}| \rightarrow \infty}{\longrightarrow} \infty$. Note that $P_{\mathbf{x}_{[L]}}^{\prime} \in \mathcal{N} \subset \mathcal{J}_{\lambda}^{\otimes L}\left(P_{\mathbf{x}}\right)$ may not be exactly a $P_{\mathbf{x}}$-self-coupling. However, by Lemma 28, there is a constant $\lambda^{\prime}=\lambda^{\prime}(\lambda)>0$ and a $P_{\mathbf{x}}$-self-coupling $P_{\mathbf{x}_{[L]}} \in \mathcal{J}^{\otimes L}\left(P_{\mathbf{x}}\right)$ such that $d\left(P_{\mathbf{x}_{[L]}}, P_{\mathbf{x}_{[L]}}^{\prime}\right) \leqslant \lambda^{\prime}$. We point out that $P_{\mathbf{x}_{[L]}}$ may be inside $\mathcal{K}_{\lambda}^{\otimes L}\left(P_{\mathbf{x}}\right)$. Nevertheless, as long as $\lambda \ll \varepsilon, P_{\mathbf{x}_{[L]}}$ is still outside $\mathrm{CP}^{\otimes L}\left(P_{\mathbf{x}}\right)$ since $\mathrm{CP}^{\otimes L}\left(P_{\mathbf{x}}\right)$ is a proper subset of $\mathcal{K}^{\otimes L}\left(P_{\mathbf{x}}\right)$ and they are $\varepsilon$-separated. Indeed,

$d\left(P_{\mathbf{x}_{[L]}}, \mathrm{CP}^{\otimes L}\left(P_{\mathbf{x}}\right)\right) \geqslant d\left(P_{\mathbf{x}_{[L]}}^{\prime}, \mathrm{CP}^{\otimes L}\left(P_{\mathbf{x}}\right)\right)-d\left(P_{\mathbf{x}_{[L]}}^{\prime}, P_{\mathbf{x}_{[L]}}\right) \geqslant d\left(\mathcal{J}^{\otimes L}\left(P_{\mathbf{x}}\right) \backslash \mathcal{K}^{\otimes L}\left(P_{\mathbf{x}}\right), \mathrm{CP}^{\otimes L}\left(P_{\mathbf{x}}\right)\right)-\lambda^{\prime} \geqslant \varepsilon-\lambda^{\prime}$.

See Figure 12 for the geometry of various sets of distributions that show up in the proof.

Define the asymmetry of a distribution $P_{\mathbf{x}_{[L]}} \in \Delta\left(\mathcal{X}^{L}\right)$ as

$$
\operatorname{asymm}\left(P_{\mathbf{x}_{[L]}}\right):=\max _{\pi \in S_{L}} \max _{x_{[L]} \in \mathcal{X}^{L}}\left|P_{\mathbf{x}_{[L]}}\left(x_{[L]}\right)-P_{\mathbf{x}_{[L]}}\left(x_{\pi([L])}\right)\right| .
$$

Given $P_{\mathbf{x}_{[L]}} \in \mathcal{J}^{\otimes L}\left(P_{\mathbf{x}}\right)$, we now consider two cases where $\operatorname{asymm}\left(P_{\mathbf{x}_{[L]}}\right) \geqslant \alpha$ and $\operatorname{asymm}\left(P_{\mathbf{x}_{[L]}}\right)<\alpha$. Let $M^{\prime}:=\left|\mathcal{C}^{\prime}\right|$ and $M:=|\mathcal{C}|$. Write $\mathcal{C}^{\prime}=\left\{\underline{x}_{i}\right\}_{i \in\left[M^{\prime}\right]}$.

a) Asymmetric case: If $P_{\mathbf{x}_{[L]}}$ has asymmetry at least $\alpha$, the code $\mathcal{C}^{\prime}$ has to be small by a result of [Kom90] and its list-decoding extension by [BD20]. Their results do not have to do with the self-coupledness of $P_{\mathbf{x}_{[L]}}$ and hence are directly reusable.

b) Symmetric case: In this case we assume $P_{\mathbf{x}_{[L]}}$ has asymmetry less than $\alpha$. We first project $P_{\mathbf{x}_{[L]}}$ to $\operatorname{Sym}^{\otimes L}\left(P_{\mathbf{x}}\right)$ to get an exactly symmetric distribution. The projection is nothing but the symmetrization of $P_{\mathbf{x}_{[L]}}$ :

$$
\bar{P}_{\mathbf{x}_{[L]}}:=\frac{1}{L !} \sum_{\pi \in S_{L}} P_{\mathbf{x}_{\pi([L])}},
$$




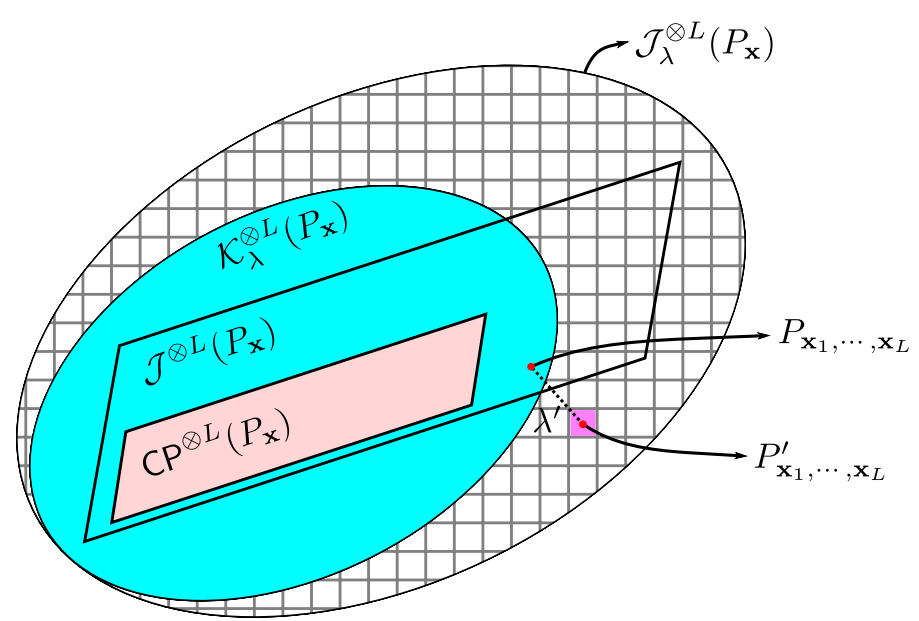

Fig. 12: The geometry of various sets of distributions in the proof of the robust generalized Plotkin bound (Theorem 29).

which is apparently symmetric. Using equicoupledness of $P_{\mathbf{x}_{[L]}}$, one can easily check the equicoupledness of $\bar{P}_{\mathbf{x}_{[L]}}$. Indeed, for any $i \in[L]$,

$$
\left[\bar{P}_{\left.\mathbf{x}_{[L]}\right]}\right]_{\mathbf{x}_{i}}=\left[\frac{1}{L !} \sum_{\pi \in S_{L}} P_{\mathbf{x}_{\pi([L])}}\right]_{\mathbf{x}_{i}}=\frac{1}{L !} \sum_{\pi \in S_{L}}\left[P_{\mathbf{x}_{\pi([L])}}\right]_{\mathbf{x}_{i}}=\frac{1}{L !} \sum_{\pi \in S_{L}}\left[P_{\mathbf{x}_{[L]}}\right]_{\mathbf{x}_{\pi^{-1}(i)}}=\frac{1}{L !} \sum_{\pi \in S_{L}} P_{\mathbf{x}}=P_{\mathbf{x}} .
$$

Therefore $\bar{P}_{\mathbf{x}_{[L]}} \in \operatorname{Sym}^{\otimes L}\left(P_{\mathbf{x}}\right)$. We also note that $\bar{P}_{\mathbf{x}_{[L]}}$ is not too far from $P_{\mathbf{x}_{[L]}}$ :

$$
\begin{aligned}
d\left(P_{\mathbf{x}_{[L]}}, \bar{P}_{\mathbf{x}_{[L]}}\right) & =\sum_{x_{[L]} \in \mathcal{X}^{L}}\left|P_{\mathbf{x}_{[L]}}\left(x_{[L]}\right)-\bar{P}_{\mathbf{x}_{[L]}}\left(x_{[L]}\right)\right| \\
& =\sum_{x_{[L]} \in \mathcal{X}^{L}}\left|\frac{1}{L !} \sum_{\pi \in S_{L}}\left(P_{\mathbf{x}_{[L]}}\left(x_{[L]}\right)-P_{\mathbf{x}_{\pi([L])}}\left(x_{[L]}\right)\right)\right| \\
& \leqslant \frac{1}{L !} \sum_{\pi \in S_{L}} \sum_{x_{[L]} \in \mathcal{X}^{L}}\left|P_{\mathbf{x}_{[L]}}\left(x_{[L]}\right)-P_{\mathbf{x}_{\pi([L])}}\left(x_{[L]}\right)\right| \\
& \leqslant\left(|\mathcal{X}|^{L}-|\mathcal{X}|\right) \alpha=: \alpha^{\prime} .
\end{aligned}
$$

By taking $\alpha^{\prime}$ to be sufficiently small, we can ensure $\bar{P}_{\mathbf{x}_{[L]}} \notin \mathrm{CP}\left(P_{\mathbf{x}}\right)$. Indeed,

$$
d\left(\bar{P}_{\mathbf{x}_{[L]},}, \mathrm{CP}^{\otimes L}\left(P_{\mathbf{x}}\right)\right) \geqslant d\left(P_{\mathbf{x}_{[L]}}, \mathrm{CP}^{\otimes L}\left(P_{\mathbf{x}}\right)\right)-d\left(P_{\mathbf{x}_{[L]}}, \bar{P}_{\mathbf{x}_{[L]}}\right) \geqslant \varepsilon-\lambda^{\prime}-\alpha^{\prime} .
$$

Since $\bar{P}_{\mathbf{x}_{[L]}} \in \mathrm{Sym}^{\otimes L}\left(P_{\mathbf{x}}\right) \backslash \mathrm{CP}{ }^{\otimes L}\left(P_{\mathbf{x}}\right)$, the duality between completely positively tensor cone and copositive tensor cone (denoted by coP $\left.{ }^{\otimes L}\left(P_{\mathbf{x}}\right)\right)^{24}$ guarantees the existence of a witness $Q_{\mathbf{x}_{[L]}} \in \operatorname{coP}^{\otimes L}\left(P_{\mathbf{x}}\right)$ of non-complete positivity. The witness satisfies that $\left\langle\bar{P}_{\mathbf{x}_{[L]}}, Q_{\mathbf{x}_{[L]}}\right\rangle \leqslant-\varepsilon^{\prime}$ for some $\varepsilon^{\prime}=\varepsilon^{\prime}\left(\varepsilon, \lambda^{\prime}\right)>0$. To get an upper bound on $M^{\prime}$ (and hence an upper bound $M$ ), we bound the following quantity from above and below: $\sum_{\mathcal{L} \in\left[M^{\prime}\right]^{L}}\left\langle\tau_{\underline{x}_{\mathcal{L}}}, Q_{\mathbf{x}_{[L]}}\right\rangle$.

On the one hand, via the method of types, it is easy to show that the above quantity is nonnegative. Indeed, it is precisely equal to

$$
\frac{M^{\prime L}}{n} \sum_{j=1}^{n}\left\langle\left(P_{\mathbf{x}}^{(j)}\right)^{\otimes L}, Q_{\mathbf{x}_{[L]}}\right\rangle \geqslant 0
$$

where $P_{\mathbf{x}}^{(j)}$ is the type of the $j$-the column of $\mathcal{C}^{\prime} \in \mathcal{X}^{M^{\prime} \times n}$. Inequality (59) follows since $\left(P_{\mathbf{x}}^{(j)}\right)^{\otimes L} \in \mathrm{CP}$ for each $j \in[n]$. See $[\mathrm{ZBJ} 20]$ for details.

${ }^{24}$ A distribution $P_{\mathbf{x}_{[L]}} \in \operatorname{Sym}{ }^{\otimes L}\left(P_{\mathbf{x}}\right)$ is called $P_{\mathbf{x}}$-copositive if $\left\langle P_{\mathbf{x}_{[L]}}, Q_{\mathbf{x}}^{\otimes L}\right\rangle \geqslant 0$ for all $Q_{\mathbf{x}} \in \Delta(\mathcal{X})$. The set of copositive distributions form a convex cone, denoted by $\operatorname{coP}^{\otimes L}\left(P_{\mathbf{x}}\right)$. It turns out that $\mathrm{CP}^{\otimes L}$ and $\operatorname{coP}^{\otimes L}$ are dual cones of each other. 
On the other hand,

$$
\sum_{\mathcal{L} \in\left[M^{\prime}\right]^{L}}\left\langle\tau_{\underline{x}_{\mathcal{L}}}, Q_{\mathbf{x}_{[L]}}\right\rangle=\sum_{\mathcal{L} \in\left(\begin{array}{c}
{\left[M^{\prime}\right]} \\
L
\end{array}\right)}\left\langle\tau_{\underline{x}_{\mathcal{L}}}, Q_{\mathbf{x}_{[L]}}\right\rangle+\sum_{\mathcal{L} \in\left[M^{\prime}\right]^{L} \backslash\left(\begin{array}{c}
{\left[M^{\prime}\right]} \\
L
\end{array}\right)}\left\langle\underline{\tau}_{\underline{x}_{\mathcal{L}}}, Q_{\mathbf{x}_{[L]}}\right\rangle .
$$

It turns out that the second term is a lower order term and it suffices to use a trivial bound:

$$
\sum_{\mathcal{L} \in\left(\begin{array}{c}
{\left[M^{\prime}\right]} \\
L
\end{array}\right)}\left\langle\tau_{\underline{x}_{\mathcal{L}}}, Q_{\mathbf{x}_{[L]}}\right\rangle \leqslant M^{\prime L}-\left(\begin{array}{c}
M^{\prime} \\
L
\end{array}\right) .
$$

We then focus on the first term. Before proceeding, we first observe that without loss of generality, we can assume $\mathcal{L}=\left\{i_{1}, \cdots, i_{L}\right\}$ is an ordered list, i.e., $i_{1}<\cdots<i_{L}$. For a list $\mathcal{L}$ that is not in ascending order, there is a $\sigma_{\mathcal{L}} \in S_{L}$ such that $\sigma_{\mathcal{L}}(\mathcal{L})$ is in ascending order. The observation then follows since

$$
\left\langle\tau_{\underline{x}_{\mathcal{L}}}, Q_{\mathbf{x}_{[L]}}\right\rangle=\left\langle\tau_{\underline{x}_{\sigma_{\mathcal{L}}(\mathcal{L})}}, Q_{\mathbf{x}_{\left.\sigma_{\mathcal{L}}(L L]\right)}}\right\rangle=\left\langle\tau_{\underline{x}_{\sigma_{\mathcal{L}}(\mathcal{L})}}, Q_{\mathbf{x}_{[L]}}\right\rangle,
$$

where the last equality is by the symmetry of $Q_{\mathbf{x}_{[L]}} \in \operatorname{coP}^{\otimes L}\left(P_{\mathbf{x}}\right) \subset \operatorname{Sym}^{\otimes L}\left(P_{\mathbf{x}}\right)$.

We now bound the first term.

$$
\begin{aligned}
& \sum_{\mathcal{L} \in\left(\begin{array}{c}
{\left[M^{\prime}\right]} \\
L
\end{array}\right)}\left\langle\tau_{\underline{x}_{\mathcal{L}}}, Q_{\mathbf{x}_{[L]}}\right\rangle=\sum_{\mathcal{L} \in\left(\begin{array}{c}
{\left[M^{\prime}\right]} \\
L
\end{array}\right)}\left\langle\tau_{\underline{x}_{\mathcal{L}}}-\bar{P}_{\mathbf{x}_{[L]}}, Q_{\mathbf{x}_{[L]}}\right\rangle+\sum_{\mathcal{L} \in\left(\begin{array}{c}
{\left[M^{\prime}\right]} \\
L
\end{array}\right)}\left\langle\bar{P}_{\mathbf{x}_{[L]}}, Q_{\mathbf{x}_{[L]}}\right\rangle \\
& \leqslant \sum_{\mathcal{L} \in\left(\begin{array}{c}
{\left[M^{\prime}\right]} \\
L
\end{array}\right)}\left\|\tau_{\underline{x}_{\mathcal{L}}}-\bar{P}_{\mathbf{x}_{[L]}}\right\|_{1} \| Q_{\mathbf{x}_{[L]} \|_{\infty}}+\sum_{\mathcal{L} \in\left(\begin{array}{c}
{\left[M^{\prime}\right]} \\
L
\end{array}\right)}\left(-\varepsilon^{\prime}\right) \\
& \leqslant \sum_{\mathcal{L} \in\left(\begin{array}{c}
{\left[M^{\prime}\right]} \\
L
\end{array}\right)}\left\|\tau_{\underline{x}_{\mathcal{L}}}-\bar{P}_{\mathbf{x}_{[L]}}\right\|_{1}-\left(\begin{array}{c}
M^{\prime} \\
L
\end{array}\right) \varepsilon^{\prime} \\
& \leqslant \sum_{\mathcal{L} \in\left(\begin{array}{c}
{\left[M_{L}^{\prime}\right]} \\
L
\end{array}\right)}\left(d\left(\tau_{\underline{x}_{\mathcal{L}}}, P_{\mathbf{x}_{[L]}}^{\prime}\right)+d\left(P_{\mathbf{x}_{[L]}}^{\prime}, P_{\mathbf{x}_{[L]}}\right)+d\left(P_{\mathbf{x}_{[L]}}, \bar{P}_{\mathbf{x}_{[L]}}\right)\right)-\left(\begin{array}{c}
M^{\prime} \\
L
\end{array}\right) \varepsilon^{\prime} \\
& \leqslant\left(\begin{array}{c}
M^{\prime} \\
L
\end{array}\right)\left(\eta+\lambda^{\prime}+\alpha^{\prime}-\varepsilon^{\prime}\right) \text {. }
\end{aligned}
$$

Combining Inequalities (59) to (61) allows us to establish Theorem 29.

Remark 17. Though the robust version of the generalized Plotkin bound allows the input distributions to be slightly perturbed from a fixed composition, in the actual double counting argument, we still made the effort to preprocess $P_{\mathbf{x}_{[L]}}^{\prime} \in \mathcal{J}_{\lambda}^{\otimes L}\left(P_{\mathbf{x}}\right)$ to get $P_{\mathbf{x}_{[L]}} \in \mathcal{J}^{\otimes L}\left(P_{\mathbf{x}}\right)$ and then $\bar{P}_{\mathbf{x}_{[L]}} \in \operatorname{Sym}^{\otimes L}\left(P_{\mathbf{x}}\right)$. This process created several slack factors that ultimately turned out to be negligible. The reason why we desire a symmetric self-coupling is at its root that the notion of complete positivity only makes sense for symmetric tensors whose marginals are all the same. After all, CP-tensors are defined to take the form $\sum_{i} \lambda_{i} P^{\otimes L}$ rather than $\sum_{i} \lambda_{i} P_{i}^{\otimes L}$ where $P_{i}$ 's can be different.

\section{APPENDIX B}

\section{CP-SYMMETRIZATION CONVERSE}

We assume that $P_{\mathbf{s}}=\left[\widetilde{P}_{\mathbf{u}} \widetilde{P}_{\mathbf{x} \mid \mathbf{u}}^{\otimes L} U_{\mathbf{s} \mid \mathbf{u}, \mathbf{x}_{1}, \cdots, \mathbf{x}_{L}}\right]_{\mathbf{s}}$ (where $\left(\widetilde{P}_{\mathbf{u}}, \widetilde{P}_{\mathbf{x} \mid \mathbf{u}}\right.$ ) was constructed in Section IX) is strictly inside $\lambda_{\mathbf{s}}$ in the sense that $P_{\mathbf{S}}$ is in the $\underline{\delta}$-interior of $\lambda_{\mathbf{s}}$ for some entry-wise positive vector $\underline{\delta}=\left(\delta_{1}, \cdots, \delta_{\beta}\right) \in \mathbb{R}_{>0}^{\beta}$. More precisely, we assume Equation (12) (which we recall below) holds: $\operatorname{cost}_{i}\left(\left(P_{\mathbf{u}}, P_{\mathbf{x} \mid \mathbf{u}}\right), U_{\mathbf{s} \mid \mathbf{u}, \mathbf{x}_{[L]}}\right) \leqslant \Lambda_{i}-\delta_{i}$ for all $i \in[\beta]$. See Figure 13 .

Under the jamming strategy described in Section IX, we lower bound the expected average error probability over the list selection and the jamming sequence generation.

Define a good event that the list chosen by James has a type that is $(\eta+\varepsilon)$-close to a certain CP-distribution:

$$
\mathcal{G}:=\left\{d\left(\tau_{\underline{\mathbf{x}}_{\mathcal{L}}}, \widetilde{P}_{\mathbf{x}_{[L]}}\right) \leqslant \eta+\varepsilon\right\} .
$$




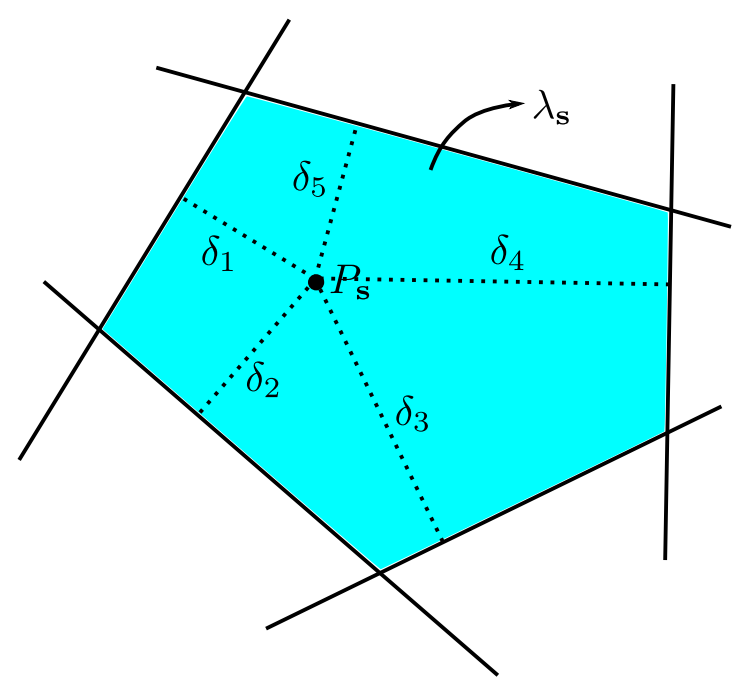

Fig. 13: We assume in CP-symmetrization that $P_{\mathbf{s}}$ is in the $\underline{\delta}$-interior of $\lambda_{\mathbf{s}}$.

Note that $\mathcal{G}$ only depends on the choice of $\mathcal{L}$ but not $\underline{\mathbf{s}}$. Then we have

$$
\begin{aligned}
\underset{\mathcal{L}, \underline{\mathbf{s}}}{\mathbb{E}}\left[P_{\mathrm{e}, \operatorname{avg}}(\underline{\mathbf{s}})\right] & =\underset{\mathcal{L}}{\underset{\mathcal{L}}{\mathbb{E}}}\left[\underset{\underline{\mathbf{s}}}{\mathbb{E}}\left[P_{\mathrm{e}, \operatorname{avg}}(\underline{\mathbf{s}}) \mid \mathcal{L}\right]\right] \geqslant \underset{\mathcal{L}}{\mathbb{E}}\left[\underset{\underline{\mathbf{s}}}{\mathbb{E}}\left[P_{\mathrm{e}, \operatorname{avg}}(\underline{\mathbf{s}}) \mathbb{1}_{\mathcal{G}} \mid \mathcal{L}\right]\right]=\underset{\mathcal{L}}{\underset{\mathcal{E}}{\mathbb{E}}}\left[\mathbb{1}_{\mathcal{G}} \underset{\underline{\mathbf{s}}}{\mathbb{E}}\left[P_{\mathrm{e}, \operatorname{avg}}(\underline{\mathbf{s}}) \mid \mathcal{L}\right]\right] \\
& =\underset{\mathcal{L}}{\operatorname{Pr}}[\mathcal{G}] \underset{\mathcal{L}}{\mathbb{E}}\left[\underset{\underline{\mathbf{s}}}{\mathbb{E}}\left[P_{\mathrm{e}, \operatorname{avg}}(\underline{\mathbf{s}}) \mid \mathcal{L}\right] \mid \mathcal{G}\right]=\underset{\mathcal{L}}{\operatorname{Pr}}[\mathcal{G}] \underset{\mathcal{L}}{\mathbb{E}}\left[\underset{\underline{\mathbf{s}}}{\mathbb{E}}\left[P_{\mathrm{e}, \operatorname{avg}}(\underline{\mathbf{s}}) \mid \mathcal{L}, \mathcal{G}\right]\right] .
\end{aligned}
$$

The expectations and probabilities are over $\mathcal{L} \sim\left(\begin{array}{c}{[M]} \\ L\end{array}\right)$ and $\underline{\mathbf{s}} \sim \prod_{j=1}^{n} U_{\mathbf{s} \mid \mathbf{u}, \mathbf{x}_{[L]}=\underline{\mathbf{x}}_{\mathcal{L}}(j)}$.

We first argue that $\operatorname{Pr}[\mathcal{G}]$ is bounded away from zero, $\operatorname{Pr}[\mathcal{G}] \geqslant c$. Let $\mathcal{F}:=\left\{d\left(\tau_{\underline{\mathbf{x}}_{\mathcal{L}}}, \widehat{P}_{\mathbf{x}_{[L]}}\right) \leqslant \eta\right\}$. As we have shown in Equation $(10), \operatorname{Pr}[\mathcal{F}] \geqslant c$. Note that by Equation $(11), \mathcal{F}$ implies

$$
d\left(\tau_{\underline{\mathbf{x}}_{\mathcal{L}}}, \widetilde{P}_{\mathbf{x}_{[L]}}\right) \leqslant d\left(\tau_{\underline{x}_{\mathcal{L}}}, \widehat{P}_{\mathbf{x}_{[L]}}\right)+d\left(\widehat{P}_{\mathbf{x}_{[L]}}, \widetilde{P}_{\mathbf{x}_{[L]}}\right) \leqslant \eta+\varepsilon
$$

Hence $\operatorname{Pr}[\mathcal{G}] \geqslant c$.

It remains to bound $\mathbb{E}\left[\mathbb{E}\left[P_{\mathrm{e}, \text { avg }}(\underline{\mathbf{s}}) \mid \mathcal{L}, \mathcal{G}\right]\right]$.

Fix any realization $1 \leqslant i_{1}<\cdots<i_{L} \leqslant M$ of the random list $\mathcal{L}$ that satisfies $\mathcal{G}$. All expectations and probabilities in the following analysis are over $\underline{\mathbf{s}}$. For any $i \in[\beta]$, the expected cost of $\underline{\mathbf{s}}$ given $\mathcal{L}$ is

$$
\begin{aligned}
& \mathbb{E}\left[B_{i}(\underline{\mathbf{s}}) \mid \mathcal{L}, \mathcal{G}\right] \\
= & \frac{1}{n} \sum_{j=1}^{n} \mathbb{E}\left[B_{i}(\underline{\mathbf{s}}(j)) \mid \mathcal{L}, \mathcal{G}\right] \\
= & \frac{1}{n} \sum_{j=1}^{n} \sum_{s \in \mathcal{S}} U_{\mathbf{s} \mid \mathbf{u}, \mathbf{x}_{[L]}}\left(s \mid \underline{u}(j), \underline{x}_{i_{1}}(j), \cdots, \underline{x}_{i_{L}}(j)\right) B_{i}(s) \\
= & \frac{1}{n} \sum_{j=1}^{n} \sum_{s \in \mathcal{S}} \sum_{u \in \mathcal{U}} \sum_{\left(x_{1}, \cdots, x_{L}\right) \in \mathcal{X}^{L}} \mathbb{1}\{\underline{u}(j)=u\} \mathbb{1}\left\{\underline{x}_{i_{1}}(j)=x_{1}, \cdots, \underline{x}_{i_{L}}(j)=x_{L}\right\} U_{\mathbf{s} \mid \mathbf{u}, \mathbf{x}_{[L]}}\left(s \mid u, x_{1}, \cdots, x_{L}\right) B_{i}(s) \\
= & \sum_{s \in \mathcal{S}} \sum_{u \in \mathcal{U}} \sum_{\left(x_{1}, \cdots, x_{L}\right) \in \mathcal{X}^{L}} U_{\mathbf{s} \mid \mathbf{u}, \mathbf{x}_{[L]}}\left(s \mid u, x_{1}, \cdots, x_{L}\right) B_{i}(s) \frac{1}{n} \sum_{j=1}^{n} \mathbb{1}\{\underline{u}(j)=u\} \mathbb{1}\left\{\underline{x}_{i_{1}}(j)=x_{1}, \cdots, \underline{x}_{i_{L}}(j)=x_{L}\right\} \\
= & \sum_{s \in \mathcal{S}} \sum_{u \in \mathcal{U}} \sum_{\left(x_{1}, \cdots, x_{L}\right) \in \mathcal{X}^{L}} U_{\mathbf{s} \mid \mathbf{u}, \mathbf{x}_{[L]}}\left(s \mid u, x_{1}, \cdots, x_{L}\right) B_{i}(s) \underline{\tau}_{u, x_{i_{1}}, \cdots, \underline{x}_{i_{L}}}\left(u, x_{1}, \cdots, x_{L}\right)
\end{aligned}
$$


The above jamming cost does not differ much from the cost computed using the joint distribution $\widetilde{P}_{\mathbf{u}} \widetilde{P}_{\mathbf{x} \mid \mathbf{u}} U_{\mathbf{s} \mid \mathbf{u}, \mathbf{x}_{[L]}}$, given the fact that $\tau_{\underline{u}, \underline{x}_{i}, \cdots, \underline{x}_{i}}$ is $\eta$-close to $\widehat{P}_{\mathbf{x}_{1}, \cdots, \mathbf{x}_{L}}$ which is in turn $\varepsilon$-close to $\widetilde{P}_{\mathbf{x}_{1}, \cdots, \mathbf{x}_{L}} \in \mathrm{CP} \otimes L\left(\widehat{P}_{\mathbf{x}}\right)$. Indeed,

$$
\begin{aligned}
& \left|\sum_{s \in \mathcal{S}} \sum_{u \in \mathcal{U}} \sum_{x_{\mathcal{L}} \in \mathcal{X}^{L}} U_{\mathbf{s} \mid \mathbf{u}, \mathbf{x}_{[L]}}\left(s \mid u, x_{\mathcal{L}}\right) B_{i}(s) \tau_{\underline{u}}, \underline{x}_{i_{1}}, \cdots, \underline{x}_{i_{L}}\left(u, x_{\mathcal{L}}\right)-\sum_{s \in \mathcal{S}} \sum_{u \in \mathcal{U}} \sum_{x_{\mathcal{L}} \in \mathcal{X}^{L}} U_{\mathbf{s} \mid \mathbf{u}, \mathbf{x}_{[L]}}\left(s \mid u, x_{\mathcal{L}}\right) B_{i}(s) \widetilde{P}_{\mathbf{u}}(u) \widetilde{P}_{\mathbf{x} \mid \mathbf{u}}^{\otimes L}\left(x_{\mathcal{L}} \mid u\right)\right| \\
= & \left|\sum_{s \in \mathcal{S}} \sum_{x_{\mathcal{L}} \in \mathcal{X}^{L}} \tau_{\underline{x}_{i_{1}}, \cdots, \underline{x}_{i_{L}}}\left(x_{\mathcal{L}}\right) U_{\mathbf{s} \mid \mathbf{x}_{[L]}}\left(s \mid x_{\mathcal{L}}\right) B_{i}(s)-\sum_{s \in \mathcal{S}} \sum_{x_{\mathcal{L}} \in \mathcal{X}^{L}} \widetilde{P}_{\mathbf{x}_{[L]}}\left(x_{\mathcal{L}}\right) U_{\mathbf{s} \mid \mathbf{x}_{[L]}}\left(s \mid x_{\mathcal{L}}\right) B_{i}(s)\right| \\
= & \sum_{s \in \mathcal{S}} \sum_{x_{\mathcal{L}} \in \mathcal{X}^{L}}\left|U_{\mathbf{s} \mid \mathbf{x}_{[L]}}\left(s \mid x_{\mathcal{L}}\right)\right|\left|B_{i}(s)\right|\left|\tau_{\underline{x}_{i_{1}}, \cdots, \underline{x}_{i_{L}}}\left(x_{\mathcal{L}}\right)-\widetilde{P}_{\mathbf{x}_{[L]}}\left(x_{\mathcal{L}}\right)\right| \\
\leqslant & \sum_{s \in \mathcal{S}} \sum_{x_{\mathcal{L}} \in \mathcal{X}^{L}}\left|B_{i}(s)\right|\left|\tau_{\underline{x}_{i_{1}}, \cdots, \underline{x}_{i_{L}}}\left(x_{\mathcal{L}}\right)-\widetilde{P}_{\mathbf{x}_{[L]}}\left(x_{\mathcal{L}}\right)\right| \\
\leqslant & |\mathcal{S}| B_{i}^{*} d\left(\tau_{\underline{x}_{i_{1}}, \cdots, \underline{x}_{i_{L}}}, \widetilde{P}_{\mathbf{x}_{[L]}}\right) \\
\leqslant & |\mathcal{S}| B_{i}^{*}(\eta+\varepsilon) .
\end{aligned}
$$

In Equation (65), $N_{i}^{*}$ is defined as $B_{i}^{*}:=\max _{s \in \mathcal{S}}\left|B_{i}(s)\right|$.

Combining Equation (64), Equation (66) and Equation (12), we get

$$
\mathbb{E}\left[B_{i}(\underline{\mathbf{s}}) \mid \mathcal{L}, \mathcal{G}\right] \leqslant \operatorname{cost}_{i}\left(\left(\widetilde{P}_{\mathbf{u}}, \widetilde{P}_{\mathbf{x} \mid \mathbf{u}}\right), U_{\mathbf{s} \mid \mathbf{u}, \mathbf{x}_{[L]}}\right)+|\mathcal{S}| B_{i}^{*}(\eta+\varepsilon) \leqslant \Lambda_{i}-\delta_{i}+|\mathcal{S}| B_{i}^{*}(\eta+\varepsilon) \leqslant \Lambda_{i}-\delta_{i} / 2<\Lambda_{i} .
$$

In the last inequality we assume $\eta \leqslant \frac{\delta_{i}}{4|\mathcal{S}| B_{i}^{*}}$ and $\varepsilon \leqslant \frac{\delta_{i}}{4|\mathcal{S}| B_{i}^{*}}$ for all $i \in[\beta]$.

We then bound the (conditional) variance of $B_{i}(\underline{\mathbf{s}})$.

$$
\begin{aligned}
\operatorname{Var}\left[B_{i}(\underline{\mathbf{s}}) \mid \mathcal{L}, \mathcal{G}\right] & =\operatorname{Var}\left[\frac{1}{n} \sum_{j=1}^{n} B_{i}(\underline{\mathbf{s}}(j)) \mid \mathcal{L}, \mathcal{G}\right] \\
& =\frac{1}{n^{2}} \sum_{j=1}^{n} \operatorname{Var}\left[B_{i}(\underline{\mathbf{s}}(j)) \mid \mathcal{L}, \mathcal{G}\right] \\
& \leqslant \frac{1}{n^{2}} \sum_{j=1}^{n} \mathbb{E}\left[B_{i}(\underline{\mathbf{s}}(j))^{2} \mid \mathcal{L}, \mathcal{G}\right] \\
& \leqslant \frac{1}{n^{2}} n\left(B_{i}^{*}\right)^{2} \\
& =\left(B_{i}^{*}\right)^{2} / n,
\end{aligned}
$$

where Equation (67) is because each component of $\underline{\mathbf{s}}$ is sampled independently. Now by Chebyshev's inequality (Lemma 3),

$$
\operatorname{Pr}\left[B_{i}(\underline{\mathbf{s}})>\Lambda_{i} \mid \mathcal{L}, \mathcal{G}\right] \leqslant \operatorname{Pr}\left[\left|B_{i}(\underline{\mathbf{s}})-\left(\Lambda_{i}-\frac{\delta_{i}}{2}\right)\right|>\frac{\delta_{i}}{2} \mid \mathcal{L}, \mathcal{G}\right] \leqslant \frac{\left(B_{i}^{*}\right)^{2}}{n\left(\delta_{i} / 2\right)^{2}}=\frac{4\left(B_{i}^{*}\right)^{2}}{n \delta_{i}^{2}} .
$$

Therefore, taking a union bound over all type constraints on the jamming sequence, we have

$$
\operatorname{Pr}\left[\tau_{\mathbf{s}} \notin \lambda_{\mathbf{s}} \mid \mathcal{L}, \mathcal{G}\right]=\operatorname{Pr}\left[\exists i \in[\beta], B_{i}(\underline{\mathbf{s}})>\Lambda_{i} \mid \mathcal{L}, \mathcal{G}\right] \leqslant \sum_{i \in[\beta]} \operatorname{Pr}\left[B_{i}(\underline{\mathbf{s}})>\Lambda_{i} \mid \mathcal{L}, \mathcal{G}\right] \leqslant \sum_{i \in[\beta]} \frac{4\left(B_{i}^{*}\right)^{2}}{n \delta_{i}^{*}}
$$

We then make several observations. For any $i \in[M]$ and $i^{\prime} \in \mathcal{L}$, we have

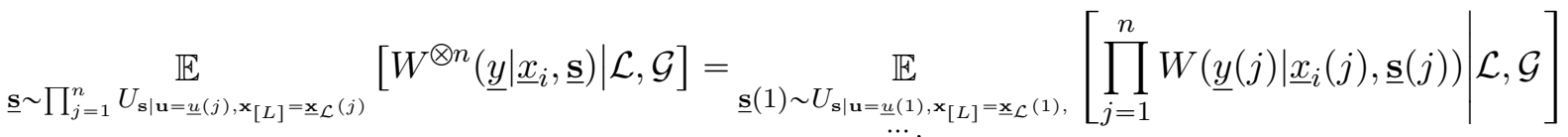

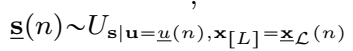

$$
\begin{aligned}
& =\prod_{j=1}^{n} \underset{\underline{\mathbf{s}}(j) \sim U_{\mathbf{s} \mid \mathbf{u}=\underline{u}(j), \mathbf{x}[L]=\underline{\mathbf{x}}_{\mathcal{L}}(j)}^{\mathbb{E}}}{\mathbb{E}}\left[W\left(\underline{y}(j) \mid \underline{x}_{i}(j), \underline{\mathbf{s}}(j)\right) \mid \mathcal{L}, \mathcal{G}\right]
\end{aligned}
$$




$$
\begin{aligned}
& =\prod_{j=1}^{n} \sum_{s \in \mathcal{S}} U_{\mathbf{s} \mid \mathbf{u}, \mathbf{x}_{[L]}}\left(s \mid \underline{u}(j), \underline{x}_{\mathcal{L}}(j)\right) W_{\mathbf{y} \mid \mathbf{x}, \mathbf{s}}\left(\underline{y}(j) \mid \underline{x}_{i}(j), s\right) \\
& =\prod_{j=1}^{n} \sum_{s \in \mathcal{S}} U_{\mathbf{s} \mid \mathbf{u}, \mathbf{x}_{[L]}}\left(s \mid \underline{u}(j), \underline{x}_{\left(\mathcal{L} \backslash\left\{i^{\prime}\right\}\right) \cup\{i\}}(j)\right) W_{\mathbf{y} \mid \mathbf{x}, \mathbf{s}}\left(\underline{y}(j) \mid \underline{x}_{i^{\prime}}(j), s\right) \\
& =\underset{\underline{\mathbf{s}} \sim \prod_{j=1}^{n} U_{\mathbf{s} \mid \mathbf{u}=\underline{u}(j), \mathbf{x}[L]=\underline{\mathbf{x}}_{\mathcal{L}}(j)}}{\mathbb{E}}\left[W^{\otimes n}\left(\underline{y} \mid \underline{x}_{i^{\prime}}, \underline{\mathbf{s}}\right) \mid\left(\mathcal{L} \backslash\left\{i^{\prime}\right\}\right) \cup\{i\}, \mathcal{G}\right] .
\end{aligned}
$$

In the above chain of equalities, Equation (68) follows since $\underline{\mathbf{s}}$ is sampled from a product distribution and the channel is memoryless $W_{\underline{\mathbf{y}} \mid \underline{\mathbf{x}}, \underline{\mathbf{s}}}=W_{\mathbf{y} \mid \mathbf{x}, \mathbf{s}}^{\otimes n}$; Equation (69) follows since $U_{\mathbf{s} \mid \mathbf{u}, \mathbf{x}_{[L]}}$ is a symmetrizing distribution (Definition 11); Equation (70) follows by rolling back the above chain of equalities.

Similar to $\mathcal{G}$, define

$$
\mathcal{G}^{\prime}:=\left\{d\left(\tau_{\underline{\mathbf{x}}_{\mathcal{L}^{\prime}}}, \widetilde{P}_{\mathbf{x}_{[L+1]}}\right) \leqslant \eta^{\prime}\right\}
$$

where $\mathcal{L}^{\prime} \sim\left(\begin{array}{c}{[M]} \\ L+1\end{array}\right)$ and $\widetilde{P}_{\mathbf{x}_{[L+1]}}:=\widetilde{P}_{\mathbf{x}_{1}, \cdots, \mathbf{x}_{L+1}} \in \mathrm{CP}^{\otimes(L+1)}\left(\widehat{P}_{\mathbf{x}}\right)$ satisfies

$$
\left[\widetilde{P}_{\mathbf{x}_{1}, \cdots, \mathbf{x}_{L+1}}\right]_{\mathbf{x}_{1}, \cdots, \mathbf{x}_{L}}=\widetilde{P}_{\mathbf{x}_{1}, \cdots, \mathbf{x}_{L}}=\widetilde{P}_{\mathbf{x}_{[L]}} .
$$

Define the set of good $L$-sized lists and good $(L+1)$-sized lists, respectively, as

$$
\mathcal{H}:=\left\{\mathcal{L} \in\left(\begin{array}{c}
{[M]} \\
L
\end{array}\right): \mathcal{G} \text { holds }\right\}, \quad \mathcal{H}^{\prime}:=\left\{\mathcal{L}^{\prime} \in\left(\begin{array}{c}
{[M]} \\
L+1
\end{array}\right): \mathcal{G}^{\prime} \text { holds }\right\} .
$$

Note that $\mathcal{G}=\{\mathcal{L} \in \mathcal{H}\}$ and $\mathcal{G}^{\prime}=\left\{\mathcal{L}^{\prime} \in \mathcal{H}^{\prime}\right\}$. Before proceeding, let us first prove the following lemma concerning the relation between $\mathcal{G}$ and $\mathcal{G}^{\prime}$.

Lemma 30. Suppose that $\mathcal{G}^{\prime}$ is defined w.r.t. a certain distribution $\widetilde{P}_{\mathbf{x}_{[L+1]}} \in \mathrm{CP} \otimes(L+1)\left(\widehat{P}_{\mathbf{x}}\right)$. Let $\eta^{\prime}>0$ in the definition of $\mathcal{G}^{\prime}$ (Equation (71)) be such that $\eta^{\prime} \leqslant \eta+\varepsilon$ and $\operatorname{Pr}_{\mathcal{L}^{\prime}}\left[\mathcal{G}^{\prime}\right] \geqslant c^{\prime} \geqslant c(L+1)$. Then $\operatorname{Pr}_{\mathcal{L}}[\mathcal{G}] \geqslant c$.

Proof. First, we claim that, if the distribution of $\mathbf{x}_{[L+1]}$ has CP-decomposition $\sum_{i \in[k]} \lambda_{i} P_{\mathbf{x}_{i}}^{\otimes(L+1)}$, then the marginal distribution of $\mathbf{x}_{[L+1] \backslash\{j\}}$ is the same for every $j \in[L+1]$ and has CP-decomposition $\sum_{i \in[k]} \lambda_{i} P_{\mathbf{x}_{i}}^{\otimes L}$. Indeed, for any $j \in[L+1]$,

$$
\begin{aligned}
& {\left[\widetilde{P}_{\left.\mathbf{x}_{[L+1]}\right]}\right]_{\mathbf{x}_{[L+1] \backslash\{j\}}}\left(x_{[L+1] \backslash\{j\}}\right)=\sum_{x_{j} \in \mathcal{X}} \sum_{i \in[k]} \lambda_{i} P_{\mathbf{x}_{i}}^{\otimes(L+1)}\left(x_{[L+1]}\right)} \\
& =\sum_{i \in[k]} \lambda_{i} \sum_{x_{j} \in \mathcal{X}} P_{\mathbf{x}_{i}}\left(x_{1}\right) \cdots P_{\mathbf{x}_{i}}\left(x_{[L+1]}\right) \\
& =\sum_{i \in[k]} \lambda_{i} P_{\mathbf{x}_{i}}^{\otimes L}\left(x_{[L+1] \backslash\{j\}}\right) \sum_{x_{j} \in \mathcal{X}} P_{\mathbf{x}_{i}}\left(x_{j}\right) \\
& =\sum_{i \in[k]} \lambda_{i} P_{\mathbf{x}_{i}}^{\otimes L}\left(x_{[L+1] \backslash\{j\}}\right) \\
& =\widetilde{P}_{\mathbf{x}_{[L]}}\left(x_{[L+1] \backslash\{j\}}\right) .
\end{aligned}
$$

Recall the definition of $\mathcal{G}$ and $\mathcal{G}^{\prime}$ in Equation (62) and Equation (71), respectively. We claim that if $\mathcal{G}^{\prime}$ holds for some $\mathcal{L}^{\prime} \in\left(\begin{array}{l}{[M]} \\ L+1\end{array}\right)$ then for any $j \in \mathcal{L}^{\prime}, \mathcal{G}$ holds, where $\mathcal{L}=\mathcal{L}^{\prime} \backslash\{j\}$. Indeed,

$$
\begin{aligned}
d\left(\tau_{\underline{x}_{\mathcal{L}}}, \widetilde{P}_{\mathbf{x}_{[L]}}\right) & =\sum_{x_{\mathcal{L}} \in \mathcal{X}^{L}}\left|\tau_{\underline{x}_{\mathcal{L}}}\left(x_{\mathcal{L}}\right)-\widetilde{P}_{\mathbf{x}_{[L]}}\left(x_{\mathcal{L}}\right)\right| \\
& =\sum_{x_{\mathcal{L}} \in \mathcal{X}^{L}}\left|\sum_{x_{j} \in \mathcal{X}} \tau_{\underline{x}_{\mathcal{L}^{\prime}}}\left(x_{\mathcal{L}^{\prime}}\right)-\widetilde{P}_{\mathbf{x}_{[L+1]}}\left(x_{\mathcal{L}^{\prime}}\right)\right| \\
& \leqslant \sum_{x_{\mathcal{L}^{\prime} \in \mathcal{X}^{L+1}}}\left|\tau_{\underline{x}_{\mathcal{L}^{\prime}}}\left(x_{\mathcal{L}^{\prime}}\right)-\widetilde{P}_{\mathbf{x}_{[L+1]}}\left(x_{\mathcal{L}^{\prime}}\right)\right|=d\left(\tau_{\underline{x}_{\mathcal{L}^{\prime}}}, \widetilde{P}_{\mathbf{x}_{[L+1]}}\right) \leqslant \eta^{\prime}
\end{aligned}
$$




$$
\leqslant \eta+\varepsilon .
$$

Equation (73) follows from the previous claim given by Equation (72). Equation (74) is by one of the assumptions $\eta^{\prime} \leqslant \eta+\varepsilon$.

By another assumption,

$$
\operatorname{Pr}_{\mathcal{L}^{\prime}}\left[\mathcal{G}^{\prime}\right]=\operatorname{Pr}_{\mathcal{L}^{\prime}}\left[\mathcal{L}^{\prime} \in \mathcal{H}^{\prime}\right]=\frac{\left|\mathcal{H}^{\prime}\right|}{\left(\begin{array}{c}
M \\
L+1
\end{array}\right)}=\frac{1}{\left(\begin{array}{c}
M \\
L+1
\end{array}\right)}\left|\left\{\mathcal{L}^{\prime} \in\left(\begin{array}{c}
{[M]} \\
L+1
\end{array}\right): d\left(\tau_{\underline{x}_{\mathcal{L}^{\prime}}}, \widetilde{P}_{\mathbf{x}_{[L+1]}}\right) \leqslant \eta^{\prime}\right\}\right| \geqslant c^{\prime} .
$$

Now we bound $\operatorname{Pr}_{\mathcal{L}}[\mathcal{G}]$.

$$
\begin{aligned}
\underset{\mathcal{L}}{\operatorname{Pr}}[\mathcal{G}] & =\frac{1}{\left(\begin{array}{c}
M \\
L
\end{array}\right)}\left|\left\{\mathcal{L} \in\left(\begin{array}{c}
{[M]} \\
L
\end{array}\right): d\left(\tau_{\underline{x}_{\mathcal{L}}}, \widetilde{P}_{\mathbf{x}_{[L]}}\right) \leqslant \eta+\varepsilon\right\}\right| \\
& \geqslant \frac{1}{\left(\begin{array}{c}
M \\
L
\end{array}\right)}\left|\left\{\mathcal{L} \in\left(\begin{array}{c}
{[M]} \\
L
\end{array}\right): \exists \mathcal{L}^{\prime} \in \mathcal{H}^{\prime}, \mathcal{L} \in\left(\begin{array}{c}
\mathcal{L}^{\prime} \\
L
\end{array}\right), d\left(\tau_{\underline{x}_{\mathcal{L}}}, \widetilde{P}_{\mathbf{x}_{[L]}}\right) \leqslant \eta+\varepsilon\right\}\right| \\
& \geqslant \frac{1}{\left(\begin{array}{c}
M \\
L
\end{array}\right)}\left|\left\{\mathcal{L} \in\left(\begin{array}{c}
{[M]} \\
L
\end{array}\right): \exists \mathcal{L}^{\prime} \in \mathcal{H}^{\prime}, \mathcal{L} \in\left(\begin{array}{c}
\mathcal{L}^{\prime} \\
L
\end{array}\right)\right\}\right| \\
& \geqslant \frac{1}{\left(\begin{array}{c}
M \\
L
\end{array}\right)} \frac{\left|\mathcal{H}^{\prime}\right|}{M-L} \\
& \geqslant \frac{c^{\prime}\left(\begin{array}{c}
M \\
L+1
\end{array}\right)}{\left(\begin{array}{c}
M \\
L
\end{array}\right)(M-L)} \\
& =\frac{c^{\prime}}{L+1} \\
& \geqslant c
\end{aligned}
$$

In Equation (76), given the condition $\mathcal{L}^{\prime} \in \mathcal{H}^{\prime}$, we could drop the condition $d\left(\tau_{\underline{x}_{\mathcal{L}}}, \widetilde{P}_{\mathbf{x}_{[L]}}\right) \leqslant \eta+\varepsilon$ since $\mathcal{G}^{\prime}$ for $\mathcal{L}^{\prime}$ implies $\mathcal{G}$ for $\mathcal{L} \in\left(\begin{array}{c}\mathcal{L}^{\prime} \\ L\end{array}\right)$, as we argued just now. Equation (77) follows from the following fact by taking $\mathscr{L}:=\mathcal{H}^{\prime}$. Fact 31. Let $\mathscr{L}^{\prime} \subseteq\left(\begin{array}{c}{[M]} \\ L+1\end{array}\right)$ be a collection of $(L+1)$-sized lists. Let $\mathscr{L}:=\left\{\mathcal{L} \in\left(\begin{array}{c}{[M]} \\ L\end{array}\right): \exists \mathcal{L}^{\prime} \in \mathscr{L}^{\prime}, \mathcal{L} \in\left(\begin{array}{c}\mathcal{L}^{\prime} \\ L\end{array}\right)\right\}$. Then $|\mathscr{L}|(M-L) \geqslant\left|\mathscr{L}^{\prime}\right|$.

Equation (78) follows from Equation (75). Equation (79) is by the assumption $\frac{c^{\prime}}{L+1} \geqslant c$.

We make another observation. For any $\mathcal{L}^{\prime} \in \mathcal{H}^{\prime}$ and $i_{0} \in \mathcal{L}^{\prime}$, we have

$$
\begin{aligned}
\sum_{i \in \mathcal{L}^{\prime}} \mathbb{E}\left[P_{\mathrm{e}, \mathrm{avg}}(i, \underline{\mathbf{s}}) \mid \mathcal{L}^{\prime} \backslash\{i\}, \mathcal{L}^{\prime} \backslash\{i\} \in \mathcal{H}\right] & =\sum_{i \in \mathcal{L}^{\prime}}\left(1-\sum_{\underline{y} \in \mathcal{Y}^{n}: \psi(\underline{y}) \ni i} \mathbb{E}\left[W^{\otimes n}\left(\underline{y} \mid \underline{x}_{i}, \underline{\mathbf{s}}\right) \mid \mathcal{L}^{\prime} \backslash\{i\}, \mathcal{L}^{\prime} \backslash\{i\} \in \mathcal{H}\right]\right) \\
& =\sum_{i \in \mathcal{L}^{\prime}}\left(1-\sum_{\underline{y} \in \mathcal{Y}^{n}: \psi(\underline{y}) \ni i} \mathbb{E}\left[W^{\otimes n}\left(\underline{y} \mid \underline{x}_{i_{0}}, \underline{\mathbf{s}}\right) \mid \mathcal{L}^{\prime} \backslash\left\{i_{0}\right\}, \mathcal{L}^{\prime} \backslash\left\{i_{0}\right\} \in \mathcal{H}\right]\right) \\
& =(L+1)-\sum_{i \in \mathcal{L}^{\prime} \cap \psi(\underline{y})} \sum_{\underline{y} \in \mathcal{Y}^{n}} \mathbb{E}\left[W^{\otimes n}\left(\underline{y} \mid \underline{x}_{i_{0}}, \underline{\mathbf{s}}\right) \mid \mathcal{L}^{\prime} \backslash\left\{i_{0}\right\}, \mathcal{L}^{\prime} \backslash\left\{i_{0}\right\} \in \mathcal{H}\right] \\
& =(L+1)-\sum_{i \in \mathcal{L}^{\prime} \cap \psi(\underline{y})} 1 \\
& \geqslant(L+1)-L \\
& =1 .
\end{aligned}
$$

Equation (80) is by Equation (70); Equation (81) follows since the inner summation equals 1; Equation (82) follows because $|\psi(\underline{y})| \leqslant L$ for any $\underline{y} \in \mathcal{Y}^{n}$. 
Given the above observations, we can lower bound $\mathbb{E}\left[\mathbb{E}\left[P_{\mathrm{e}, \text { avg }}(\underline{\mathbf{s}}) \mid \mathcal{L}, \mathcal{G}\right]\right]$ as follows.

$$
\begin{aligned}
\mathbb{E}\left[\mathbb{E}\left[P_{\mathrm{e}, \mathrm{avg}}(\underline{\mathbf{s}})\right] \mid \mathcal{L}, \mathcal{G}\right] & =\frac{1}{\left(\begin{array}{c}
M \\
L
\end{array}\right)} \frac{1}{M} \sum_{\mathcal{L} \in\left(\begin{array}{c}
{[M]} \\
L
\end{array}\right)} \sum_{i \in[M]} \mathbb{E}\left[P_{\mathrm{e}, \mathrm{avg}}(i, \underline{\mathbf{s}}) \mid \mathcal{L}\right] \mathbb{1}\{\mathcal{L} \in \mathcal{H}\} \\
& \geqslant \frac{1}{\left(\begin{array}{c}
M \\
L
\end{array}\right)} \frac{1}{M} \sum_{\mathcal{L} \in\left(\begin{array}{c}
{[M]} \\
L
\end{array}\right)} \sum_{i \in[M]: \mathcal{L} \cup\{i\} \in \mathcal{H}^{\prime}} \mathbb{E}\left[P_{\mathrm{e}, \mathrm{avg}}(i, \underline{\mathbf{s}}) \mid \mathcal{L}\right] \mathbb{1}\{\mathcal{L} \in \mathcal{H}\} \\
& \geqslant \frac{1}{\left(\begin{array}{c}
M \\
L
\end{array}\right)} \frac{1}{M} \sum_{\mathcal{L}^{\prime} \in \mathcal{H}^{\prime}} \sum_{i \in \mathcal{L}^{\prime}} \mathbb{E}\left[P_{\mathrm{e}, \mathrm{avg}}(i, \underline{\mathbf{s}}) \mid \mathcal{L}^{\prime} \backslash\{i\}\right] \mathbb{1}\left\{\mathcal{L}^{\prime} \backslash\{i\} \in \mathcal{H}\right\} \\
& =\frac{1}{\left(\begin{array}{c}
M \\
L
\end{array}\right)} \frac{1}{M} \sum_{\mathcal{L}^{\prime} \in \mathcal{H}^{\prime}} \sum_{i \in \mathcal{L}^{\prime}} \mathbb{E}\left[P_{\mathrm{e}, \mathrm{avg}}(i, \underline{\mathbf{s}}) \mid \mathcal{L}^{\prime} \backslash\{i\}\right] \\
& \geqslant \frac{1}{\left(\begin{array}{c}
M \\
L
\end{array}\right)} \frac{1}{M}\left|\mathcal{H}^{\prime}\right| \\
& \geqslant \frac{c^{\prime}\left(\begin{array}{c}
M \\
L+1
\end{array}\right)}{\left(\begin{array}{c}
M \\
L
\end{array}\right) M} \\
& =\frac{M-L}{L+1} \frac{c^{\prime}}{M} \\
& =c^{\prime}\left(\frac{1}{L+1}-\frac{L}{(L+1) M}\right) \\
& \geqslant \frac{c^{\prime}}{2(L+1)} .
\end{aligned}
$$

Since $\mathcal{L}^{\prime} \backslash\{i\} \in \mathcal{H}$ is always true given the choice of $\mathcal{L}^{\prime}$ and $i$, we can drop the indicator in Equation (84). In Equation (85), we drop the indicator of the event $\mathcal{L}^{\prime} \backslash\{i\} \in \mathcal{H}$ since $\mathcal{G}^{\prime}=\left\{\mathcal{L}^{\prime} \in \mathcal{H}^{\prime}\right\}$ automatically implies $\mathcal{G}=\left\{\mathcal{L}^{\prime} \backslash\{i\} \in \mathcal{H}\right\}$ by Lemma 30. Equation (86) follows from Equation (83). Equation (87) follows from a bound similar to $\operatorname{Pr}[\mathcal{G}] \geqslant c$. Indeed, by replacing $L$ with $L+1$, we have

$$
\operatorname{Pr}_{\mathcal{L}^{\prime} \sim\left(\begin{array}{c}
{[M]} \\
L+1
\end{array}\right)}\left[\mathcal{G}^{\prime}\right]=\underset{\mathcal{L}^{\prime} \sim\left(\begin{array}{c}
{[M]} \\
L+1
\end{array}\right)}{\operatorname{Pr}}\left[\mathcal{L}^{\prime} \in \mathcal{H}^{\prime}\right]=\frac{\left|\mathcal{H}^{\prime}\right|}{\left(\begin{array}{c}
M \\
L+1
\end{array}\right)} \geqslant c^{\prime}
$$

for some constant $c^{\prime}>0$. In Equation (88), we assume $M \geqslant 2 L$.

Finally, putting Equation (88) back to Equation (63), we have

$$
\underset{\mathcal{L}, \underline{\mathbf{s}}}{\mathbb{E}}\left[P_{\mathrm{e}, \mathrm{avg}}(\underline{\mathbf{s}})\right] \geqslant \frac{c c^{\prime}}{2(L+1)}
$$

Equation (89) finishes the proof of the symmetrization converse (Theorem 12).

APPENDIX C

\section{CODEWORD SELECTION}

Fix rate $R$ and a time-sharing sequence $\underline{u} \in \mathcal{U}^{n}$ of type $P_{\mathbf{u}}$. We sample $M=L 2^{n R}$ codewords $\underline{\mathbf{x}}_{1}, \cdots, \underline{\mathbf{x}}_{M}$ independently using the following distribution. For each $i \in[M]$, let $\underline{\mathbf{x}}_{i}^{(j)}$ denote the $j$-th $(j \in \mathcal{U})$ subvector of $\underline{\mathbf{x}}_{i}$, i.e., $\underline{\mathbf{x}}_{i}^{(j)}:=\left(\underline{\mathbf{x}}_{i}\left(k_{1}\right), \cdots, \underline{\mathbf{x}}_{i}\left(k_{n P_{\mathbf{u}}(j)}\right)\right) \in \mathcal{X}^{n P_{\mathbf{u}}(j)}$ where $\left(k_{1}, \cdots, k_{n P_{\mathbf{u}}(j)}\right)$ satisfies $\underline{\mathbf{u}}\left(k_{\ell}\right)=j$ for all $\ell \in\left[n P_{\mathbf{u}}(j)\right]$. We sample $\underline{\mathbf{x}}_{i}^{(j)}$ uniformly from all $\mathcal{X}^{n P_{\mathbf{u}}(j)}$-valued vectors of type $P_{\mathbf{x} \mid \mathbf{u}=j}$.

We will show that a codebook $\mathcal{C}$ sampled as above simultaneously satisfies all desired properties in Lemma 19 with high probability. To this end, we need the following lemma whose proof appeared in [CN88b].

Lemma 32. Let $X_{1}, \cdots, X_{M}$ be (not necessarily identically distributed, possibly dependent) random variables. For all $i \in[M]$, let $f_{i}\left(X_{1}, \cdots, X_{i}\right)$ be a function such that $f_{i} \in[0,1]$. If $\mathbb{E}\left[f_{i}\left(X_{1}, \cdots, X_{i}\right) \mid X_{1}, \cdots, X_{i-1}\right] \leqslant a$ a.s. for all $i \in[M]$, then

$$
\operatorname{Pr}\left[\frac{1}{M} \sum_{i=1}^{M} f_{i}\left(X_{1}, \cdots, X_{i}\right)>t\right] \leqslant 2^{-M(t-a \log e)} .
$$


Proof of Lemma 19.. Fix $\underline{u} \in \mathcal{U}^{n}, \underline{x} \in \mathcal{X}^{n}, \underline{s} \in \mathcal{S}^{n}, P_{\mathbf{u}} \in \Delta(\mathcal{U}), P_{\mathbf{x} \mid \mathbf{u}} \in \Delta(\mathcal{X} \mid \mathcal{U}), P_{\mathbf{u}, \mathbf{x}, \mathbf{x}[L], \mathbf{s}} \in \Delta\left(\mathcal{U} \times \mathcal{X} \times \mathcal{X}^{L} \times \mathcal{S}\right)$. To avoid trivialities, assume $P_{\mathbf{u}, \mathbf{x}, \mathbf{s}}=\tau_{\underline{u}, \underline{x}, \underline{s}}, P_{\mathbf{u}, \mathbf{x}}=P_{\mathbf{u}, \mathbf{x}_{k}}=P_{\mathbf{u}} P_{\mathbf{x} \mid \mathbf{u}}$ for all $k \in[L]$. Let $\underline{\mathbf{x}}_{1}, \cdots, \underline{\mathbf{x}}_{M}$ be i.i.d. random vectors following the distribution specified in the beginning of Appendix $\mathrm{C}$.

a) Proof of Equation (15): Fix any $k \in[L]$. Let $f_{i}\left(\underline{\mathbf{x}}_{1}, \cdots, \underline{\mathbf{x}}_{i}\right)=\mathbb{1}\left\{\tau_{\underline{u}, \underline{x}, \underline{\mathbf{x}}_{i}, \underline{s}}=P_{\mathbf{u}, \mathbf{x}, \mathbf{x}_{k}, \mathbf{s}}\right\}, i \in[M]$. Note that each $f_{i}$ in fact only depends on $\underline{\mathbf{x}}_{i}$.

$$
\begin{aligned}
a & =\mathbb{E}\left[f_{i}\left(\underline{\mathbf{x}}_{1}, \cdots, \underline{\mathbf{x}}_{i}\right) \mid \underline{\mathbf{x}}_{1}, \cdots, \underline{\mathbf{x}}_{i-1}\right] \\
& =\mathbb{E}\left[f_{i}\left(\underline{\mathbf{x}}_{1}, \cdots, \underline{\mathbf{x}}_{i}\right)\right] \\
& =\operatorname{Pr}\left[\tau_{\underline{u}, \underline{x}, \underline{\mathbf{x}}_{i}, \underline{s}}=P_{\mathbf{u}, \mathbf{x}, \mathbf{x}_{k}, \mathbf{s}}\right] \\
& =\frac{\left|\left\{\underline{x}^{\prime} \in \mathcal{X}^{n}: \tau_{\underline{u}, \underline{x}, \underline{x}^{\prime}, \underline{s}}=P_{\mathbf{u}, \mathbf{x}, \mathbf{x}_{k}, \mathbf{s}}\right\}\right|}{\left|\left\{\underline{x}^{\prime} \in \mathcal{X}^{n}: \tau_{\underline{u}, \underline{x}^{\prime}}=P_{\mathbf{u}, \mathbf{x}}\right\}\right|} \\
& \doteq \frac{2^{n H\left(\mathbf{x}_{k} \mid \mathbf{u}, \mathbf{x}, \mathbf{s}\right)}}{2^{n H(\mathbf{x} \mid \mathbf{u})}} \\
& =2^{-n\left(H\left(\mathbf{x}_{k} \mid \mathbf{u}\right)-H\left(\mathbf{x}_{k} \mid \mathbf{u}, \mathbf{x}, \mathbf{s}\right)\right)} \\
& =2^{-n I\left(\mathbf{x}_{k} ; \mathbf{x}, \mathbf{s} \mid \mathbf{u}\right)},
\end{aligned}
$$

where Equation (90) follows since $P_{\mathbf{u}, \mathbf{x}}=P_{\mathbf{u}, \mathbf{x}_{k}}$.

Let $t=\frac{1}{M} 2^{n\left(\left[R-I\left(\mathbf{x}_{k} ; \mathbf{x}, \mathbf{s} \mid \mathbf{u}\right)\right]^{+}+\varepsilon\right)}$. Under these choices of parameters $(t$ and $a)$,

$$
\begin{aligned}
M(t-a \log e) & \doteq 2^{n\left(\left[R-I\left(\mathbf{x}_{k} ; \mathbf{x}, \mathbf{s} \mid \mathbf{u}\right)\right]^{+}+\varepsilon\right)}-L 2^{n R} \log e 2^{-I\left(\mathbf{x}_{k} ; \mathbf{x}, \mathbf{s} \mid \mathbf{u}\right)} \\
& = \begin{cases}2^{n\left(R-I\left(\mathbf{x}_{k} ; \mathbf{x}, \mathbf{s} \mid \mathbf{u}\right)+\varepsilon\right)}-2^{R-I\left(\mathbf{x}_{k} ; \mathbf{x}, \mathbf{s} \mid \mathbf{u}\right)} L \log e, & R \geqslant I\left(\mathbf{x}_{k} ; \mathbf{x}, \mathbf{s} \mid \mathbf{u}\right) \\
2^{n \varepsilon}-2^{n\left(R-I\left(\mathbf{x}_{k} ; \mathbf{x}, \mathbf{s} \mid \mathbf{u}\right)\right)}, & R<I\left(\mathbf{x}_{k} ; \mathbf{x}, \mathbf{s} \mid \mathbf{u}\right)\end{cases} \\
& \geqslant 2^{n \varepsilon}
\end{aligned}
$$

for sufficiently large $n$.

Now by Lemma 32,

$$
\begin{aligned}
\operatorname{Pr}\left[\sum_{i=1}^{M} f_{i}\left(\underline{\mathbf{x}}_{1}, \cdots, \underline{\mathbf{x}}_{i}\right)>t\right] & =\operatorname{Pr}\left[\left|\left\{i \in[M]: \tau_{\underline{u}, \underline{x}, \underline{\mathbf{x}}_{i}, \underline{s}}=P_{\mathbf{u}, \mathbf{x}, \mathbf{x}_{k}, \mathbf{s}}\right\}\right|>2^{n\left(\left[R-I\left(\mathbf{x}_{k} ; \mathbf{x}, \mathbf{s} \mid \mathbf{u}\right)\right]^{+}+\varepsilon\right)}\right] \\
& \leqslant 2^{-M(a-t \log e)} \\
& \leqslant 2^{-2^{n \varepsilon}} .
\end{aligned}
$$

b) Proof of Equation (13): Following the same procedures as in the previous paragraph, we have

$$
\operatorname{Pr}\left[\left|\left\{i \in[M]: \tau_{\underline{u}, \underline{\mathbf{x}}_{i}, \underline{\underline{s}}}=P_{\mathbf{u}, \mathbf{x}, \mathbf{s}}\right\}\right|>2^{n\left([R-I(\mathbf{x} ; \mathbf{s} \mid \mathbf{u})]^{+}+\left(\varepsilon / 2+\frac{1}{n} \log L\right)\right)}\right] \leqslant 2^{-2^{n\left(\varepsilon / 2+\frac{1}{n} \log L\right)}}=2^{-L 2^{n \varepsilon / 2}} .
$$

Recall that $R \geqslant \varepsilon$. Under the assumption that $I(\mathbf{x} ; \mathbf{s} \mid \mathbf{u}) \geqslant \varepsilon$, we have

$$
[R-I(\mathbf{x} ; \mathbf{s} \mid \mathbf{u})]^{+}=R-\min \{R, I(\mathbf{x} ; \mathbf{s} \mid \mathbf{u})\} \leqslant R-\varepsilon .
$$

Hence

$$
\begin{aligned}
2^{-L 2^{n \varepsilon / 2}} & \geqslant \operatorname{Pr}\left[\left|\left\{i \in[M]: \tau_{\underline{u}, \underline{\mathbf{x}}_{i}, \underline{\underline{s}}}=P_{\mathbf{u}, \mathbf{x}, \mathbf{s}}\right\}\right|>L 2^{n\left([R-I(\mathbf{x} ; \mathbf{s} \mid \mathbf{u})]^{+}+\varepsilon / 2\right)}\right] \\
& \geqslant \operatorname{Pr}\left[\left|\left\{i \in[M]: \tau_{\underline{u}, \underline{\mathbf{x}}_{i}, \underline{s}}=P_{\mathbf{u}, \mathbf{x}, \mathbf{s}}\right\}\right|>L 2^{n(R-\varepsilon / 2)}\right] \\
& =\operatorname{Pr}\left[\frac{1}{M}\left|\left\{i \in[M]: \tau_{\underline{u}^{\prime}, \underline{\mathbf{x}}_{i}, \underline{s}}=P_{\mathbf{u}, \mathbf{x}, \mathbf{s}}\right\}\right|>2^{-n \varepsilon / 2}\right],
\end{aligned}
$$

where Equation (93) is by Equation (92). 
c) Proof of Equation (17): First observe that

$$
\begin{aligned}
\left\{\mathcal{L} \in\left(\begin{array}{c}
{[M]} \\
L
\end{array}\right): \tau_{\underline{u}, \underline{x}, \underline{\mathbf{x}}_{\mathcal{L}}, \underline{s}}=P_{\mathbf{u}, \mathbf{x}, \mathbf{x}_{[L]}, \mathbf{s}}\right\} & \subseteq\left\{\left(i_{1}, \cdots, i_{L}\right) \in\left(\begin{array}{c}
{[M]} \\
L
\end{array}\right): \tau_{\underline{u}, \underline{x}, \underline{x}_{i_{1}}, \underline{s}}=P_{\mathbf{u}, \mathbf{x}, \mathbf{x}_{1}, \mathbf{s}}, \cdots, \tau_{\underline{u}, \underline{x}, \mathbf{x}_{i_{L}, \mathbf{s}}}=P_{\mathbf{u}, \mathbf{x}, \mathbf{x}_{L}, \mathbf{s}}\right\} \\
& \subseteq\left\{\left(i_{1}, \cdots, i_{L}\right) \in[M]^{L}: \tau_{\underline{u}, \underline{x}, \underline{x}_{i_{1}}, \underline{s}}=P_{\mathbf{u}, \mathbf{x}, \mathbf{x}_{1}, \mathbf{s}}, \cdots, \tau_{\underline{u}, \underline{x}, \mathbf{x}_{i_{L}}, \mathbf{s}}=P_{\mathbf{u}, \mathbf{x}, \mathbf{x}_{L}, \mathbf{s}}\right\} \\
& =\underset{k=1}{X}\left\{i \in[M]: \tau_{\underline{u}, \underline{x}, \underline{\mathbf{x}}_{i}, \underline{s}}=P_{\mathbf{u}, \mathbf{x}, \mathbf{x}_{k}, \mathbf{s}}\right\} .
\end{aligned}
$$

Therefore,

$$
\begin{aligned}
\operatorname{Pr}\left[\left|\left\{\mathcal{L} \in\left(\begin{array}{c}
{[M]} \\
L
\end{array}\right): \tau_{\underline{u}, \underline{x}^{,} \underline{\mathbf{x}}_{\mathcal{L}}, \underline{s}}=P_{\mathbf{u}, \mathbf{x}, \mathbf{x}_{[L]}, \mathbf{s}}\right\}\right|>2^{n \varepsilon}\right] & \leqslant \operatorname{Pr}\left[\prod_{k \in[L]}\left|\left\{i \in[M]: \tau_{\underline{u}, \underline{x}, \underline{\mathbf{x}}_{i}, \underline{s}}=P_{\mathbf{u}, \mathbf{x}, \mathbf{x}_{k}, \mathbf{s}}\right\}\right|>2^{n \varepsilon}\right] \\
& \leqslant \operatorname{Pr}\left[\exists k \in[L],\left|\left\{i \in[M]: \tau_{\underline{u}, \underline{x}, \underline{\mathbf{x}}_{i}, \underline{s}}=P_{\mathbf{u}, \mathbf{x}, \mathbf{x}_{k}, \mathbf{s}}\right\}\right|>2^{n \varepsilon / L}\right] \\
& \leqslant \sum_{k \in[L]} \operatorname{Pr}\left[\left|\left\{i \in[M]: \tau_{\underline{u}, \underline{x}, \underline{\mathbf{x}}_{i}, \underline{s}}=P_{\mathbf{u}, \mathbf{x}, \mathbf{x}_{k}, \mathbf{s}}\right\}\right|>2^{n \varepsilon / L}\right] \\
& \leqslant L 2^{-2^{n \varepsilon / L}}
\end{aligned}
$$

where the last inequality follows from Equation (91) since $R<\min _{k \in[L]} I\left(\mathbf{x}_{k} ; \mathbf{s} \mid \mathbf{u}\right) \leqslant \min _{k \in[L]} I\left(\mathbf{x}_{k} ; \mathbf{x}, \mathbf{s} \mid \mathbf{u}\right)$.

d) Proof of Equation (16): We first make several definitions.

$$
\begin{aligned}
& \mathscr{L}_{i}^{\prime}:=\left\{\mathcal{L} \in\left(\begin{array}{c}
{[i-1]} \\
L
\end{array}\right): \tau_{\underline{u}, \underline{\mathbf{x}}_{\mathcal{L}}, \underline{s}}=P_{\mathbf{u}, \mathbf{x}_{[L]}, \mathbf{s}}\right\}, \\
& \mathscr{L}_{i}:=\left\{\begin{array}{ll}
\mathscr{L}_{i}^{\prime}, & \left|\mathscr{L}_{i}^{\prime}\right| \leqslant 2^{n \varepsilon_{1}} \\
\varnothing, & \left|\mathscr{L}_{i}^{\prime}\right|>2^{n \varepsilon_{1}}
\end{array},\right. \\
& f_{i}\left(\underline{\mathbf{x}}_{1}, \cdots, \underline{\mathbf{x}}_{i}\right):=\mathbb{1}\left\{\exists \mathcal{L} \in \mathscr{L}_{i}, \tau_{\underline{u}, \underline{\mathbf{x}}_{i}, \underline{\mathbf{x}}_{\mathcal{L}}, \underline{s}}=P_{\mathbf{u}, \mathbf{x}, \mathbf{x}_{[L]}, \mathbf{s}}\right\},
\end{aligned}
$$

where $\varepsilon_{1}>0$ is a small constant to be specified later.

Observe that if

$$
\max _{i \in[M]}\left|\mathscr{L}_{i}^{\prime}\right| \leqslant\left|\left\{\mathcal{L} \in\left(\begin{array}{c}
{[M]} \\
L
\end{array}\right): \tau_{\underline{u}, \underline{\mathbf{x}}_{\mathcal{L}}, \underline{s}}=P_{\mathbf{u}, \mathbf{x}_{[L]}, \mathbf{s}}\right\}\right| \leqslant 2^{n \varepsilon_{1}},
$$

then $\mathscr{L}_{i}=\mathscr{L}_{i}^{\prime}$ for all $i \in[M]$. Furthermore, under the condition in Equation (96), we have

$$
\begin{aligned}
\sum_{i \in[M]} f_{i}\left(\underline{\mathbf{x}}_{1}, \cdots, \underline{\mathbf{x}}_{i}\right) & =\sum_{i \in[M]} \mathbb{1}\left\{\exists \mathcal{L} \in \mathscr{L}_{i}, \tau_{\underline{u}, \underline{\mathbf{x}}_{i}, \underline{\mathbf{x}}_{\mathcal{L}}, \underline{s}}=P_{\mathbf{u}, \mathbf{x}, \mathbf{x}_{[L]}, \mathbf{s}}\right\} \\
& =\sum_{i \in[M]} \mathbb{1}\left\{\exists \mathcal{L} \in \mathscr{L}_{i}^{\prime}, \tau_{\underline{u}, \underline{\mathbf{x}}_{i}, \underline{\mathbf{x}}_{\mathcal{L}}, \underline{s}}=P_{\mathbf{u}, \mathbf{x}, \mathbf{x}_{[L]}, \mathbf{s}}\right\} \\
& =\sum_{i \in[M]} \mathbb{1}\left\{\exists \mathcal{L} \in\left(\begin{array}{c}
{[i-1]} \\
L
\end{array}\right), \tau_{\underline{u}, \underline{\mathbf{x}}_{\mathcal{L}}, \underline{s}}=P_{\mathbf{u}, \mathbf{x}_{[L]}, \mathbf{s}}, \tau_{\underline{u}, \underline{\mathbf{x}}_{i}, \underline{\mathbf{x}}_{\mathcal{L}}, \underline{\mathbf{s}}}=P_{\mathbf{u}, \mathbf{x}, \mathbf{x}_{[L]}, \mathbf{s}}\right\} \\
& =\sum_{i \in[M]} \mathbb{1}\left\{\exists \mathcal{L} \in\left(\begin{array}{c}
{[i-1]} \\
L
\end{array}\right), \tau_{\underline{u}, \underline{\mathbf{x}}_{i}, \underline{\mathbf{x}}_{\mathcal{L}}, \underline{\mathbf{s}}}=P_{\mathbf{u}, \mathbf{x}, \mathbf{x}_{[L]}, \mathbf{s}}\right\} \\
& =\mid\left\{i \in[M]: \exists \mathcal{L} \in\left(\begin{array}{c}
{[i-1]} \\
L
\end{array}\right), \tau_{\underline{u}, \underline{\mathbf{x}}_{i}, \underline{\mathbf{x}}_{\mathcal{L}}, \underline{s}}=P_{\left.\mathbf{u}, \mathbf{x}, \mathbf{x}_{[L]}, \mathbf{s}\right\}}\right\} .
\end{aligned}
$$

We can bound the probability that both sides of Equation (97) differ using Equation (95).

$$
\begin{aligned}
& \operatorname{Pr}\left[\left|\left\{i \in[M]: \exists \mathcal{L} \in\left(\begin{array}{c}
{[i-1]} \\
L
\end{array}\right), \tau_{\underline{u}_{\underline{\mathbf{x}}}, \underline{\mathbf{x}}_{\mathcal{L}}, \underline{s}}=P_{\mathbf{u}, \mathbf{x}, \mathbf{x}_{[L]}, \mathbf{s}}\right\}\right| \neq \sum_{i \in[M]} f_{i}\left(\underline{\mathbf{x}}_{1}, \cdots, \underline{\mathbf{x}}_{i}\right)\right] \\
= & \operatorname{Pr}\left[\exists i \in[M], \mathscr{L}_{i} \neq \mathscr{L}_{i}^{\prime}\right]
\end{aligned}
$$




$$
\begin{aligned}
& =\operatorname{Pr}\left[\exists i \in[M],\left|\left\{\mathcal{L} \in\left(\begin{array}{c}
{[i-1]} \\
L
\end{array}\right): \tau_{\underline{u}^{,} \underline{\mathbf{x}}_{\mathcal{L}}, \underline{s}}=P_{\mathbf{u}, \mathbf{x}_{[L]}, \mathbf{s}}\right\}\right|>2^{n \varepsilon_{1}}\right] \\
& \leqslant \operatorname{Pr}\left[\exists i \in[M],\left|\left\{\mathcal{L} \in\left(\begin{array}{c}
{[M]} \\
L
\end{array}\right): \tau_{\underline{u}, \underline{\mathbf{x}}_{\mathcal{L}}, \underline{s}}=P_{\mathbf{u}, \mathbf{x}_{[L]}, \mathbf{s}}\right\}\right|>2^{n \varepsilon_{1}}\right] \\
& \leqslant L 2^{-2^{n \varepsilon_{1} / L}} .
\end{aligned}
$$

We then concentrate $\sum_{i \in[M]} f_{i}\left(\underline{\mathbf{x}}_{1}, \cdots, \underline{\mathbf{x}}_{i}\right)$ using Lemma 32. To this end, let us compute

$$
\begin{aligned}
\mathbb{E}\left[f_{i}\left(\mathbf{x}_{1}, \cdots, \mathbf{x}_{i}\right) \mid \mathbf{x}_{1}, \cdots, \mathbf{x}_{i-1}\right] & =\operatorname{Pr}\left[\exists \mathcal{L} \in \mathscr{L}_{i}, \tau_{\underline{u}, \underline{\mathbf{x}}_{i}, \underline{\mathbf{x}}_{\mathcal{L}}, \underline{s}}=P_{\mathbf{u}, \mathbf{x}, \mathbf{x}_{[L]}, \mathbf{s}} \mid \underline{\mathbf{x}}_{1}, \cdots, \underline{\mathbf{x}}_{i-1}\right] \\
& \leqslant\left|\mathscr{L}_{i}\right| 2^{n H\left(\mathbf{x} \mid \mathbf{u}, \mathbf{x}_{[L]}, \mathbf{s}\right)} / 2^{n H(\mathbf{x} \mid \mathbf{u})} \\
& \leqslant 2^{-n\left(I\left(\mathbf{x} ; \mathbf{x}_{[L]}, \mathbf{s} \mid \mathbf{u}\right)-\varepsilon_{1}\right)} \\
& \leqslant 2^{-n(\varepsilon-\varepsilon / 4)}=2^{-\frac{3}{4} n \varepsilon},
\end{aligned}
$$

where Equation (100) follows from the assumption $I\left(\mathbf{x} ; \mathbf{x}_{[L]}, \mathbf{s} \mid \mathbf{u}\right) \geqslant \varepsilon$ and the choice of parameter $\varepsilon_{1}=\varepsilon / 4$. By Lemma 32, we have

$$
\operatorname{Pr}\left[\frac{1}{M} \sum_{i \in[M]} f_{i}\left(\mathbf{x}_{1}, \cdots, \mathbf{x}_{i}\right)>2^{-\frac{2}{3} n \varepsilon}\right] \leqslant 2^{-M\left(2^{-\frac{2}{3} n \varepsilon}-2^{-\frac{3}{4} n \varepsilon} \log e\right)} \leqslant 2^{-\frac{M}{2} 2^{-\frac{2}{3} n \varepsilon}} \leqslant 2^{-\frac{L}{2} 2^{n \varepsilon} 2^{-\frac{2}{3} n \varepsilon}}=2^{-\frac{L}{2} 2^{n \varepsilon / 3}},
$$

where we used the assumption $R=\frac{1}{n} \log \frac{M}{L} \geqslant \varepsilon$.

Combining Equation (99) and Equation (101), we get

$\operatorname{Pr}\left[\frac{1}{M}\left|\left\{i \in[M]: \exists \mathcal{L} \in\left(\begin{array}{c}{[i-1]} \\ L\end{array}\right), \tau_{\underline{u}, \underline{\mathbf{x}}_{i}, \underline{\mathbf{x}}_{\mathcal{L}}, \underline{s}}=P_{\mathbf{u}, \mathbf{x}, \mathbf{x}_{[L]}, \mathbf{s}}\right\}\right|>2^{-\frac{2}{3} n \varepsilon}\right] \leqslant$ Equation (98) + LHS of Equation (101) $\leqslant L 2^{-2^{\frac{n \varepsilon}{4 L}}}+2^{-\frac{L}{2} 2^{n \varepsilon / 3}} \leqslant(L+1) 2^{-2^{\frac{n \varepsilon}{4 L}}}$.

Take any $\pi \in S_{M}$. Equation (102) remains true when $\left(\begin{array}{c}{[i-1]} \\ L\end{array}\right)$ is replaced by

$$
\pi\left(\begin{array}{c}
{[i-1]} \\
L
\end{array}\right):=\left(\begin{array}{c}
\pi^{-1}[\pi(i)-1] \\
L
\end{array}\right)=\left\{L \in\left(\begin{array}{c}
{[M] \backslash\{i\}} \\
L
\end{array}\right): \forall j \in \mathcal{L}, \pi(j) \leqslant \pi(i)-1\right\} .
$$

Indeed, the proof follows by replacing $\underline{\mathbf{x}}_{i}$ with $\underline{\mathbf{x}}_{\pi(i)}$. Let $\pi_{1}, \cdots, \pi_{K}$ be permutations given by the following lemma.

Lemma 33 (Lemma A2, [Hug97]). For $n \geqslant \log (2 L)$, there exist $K\left(K \leqslant n(L+1)^{2}(\log |\mathcal{X}|+1)\right)$ permutations $\pi_{1}, \cdots, \pi_{K} \in S_{M}\left(M=L 2^{n R}\right)$ such that for all $i \in[M]$,

$$
\left(\begin{array}{c}
{[M] \backslash\{i\}} \\
L
\end{array}\right)=\bigcup_{k \in[K]} \pi_{k}\left(\begin{array}{c}
{[i-1]} \\
L
\end{array}\right)
$$

Note that for sufficiently large $n, K \leqslant 2^{n \varepsilon / 6}$. We are finally ready to prove Equation (16).

$$
\begin{aligned}
& \operatorname{Pr}\left[\frac{1}{M}\left|\left\{i \in[M]: \exists \mathcal{L} \in\left(\begin{array}{c}
{[M] \backslash\{i\}} \\
L
\end{array}\right), \tau_{\underline{u}, \underline{\mathbf{x}}_{i}, \underline{\mathbf{x}}_{\mathcal{L}}, \underline{\underline{s}}}=P_{\mathbf{u}, \mathbf{x}, \mathbf{x}_{[L]}, \mathbf{s}}\right\}\right|>2^{-n \varepsilon / 2}\right] \\
= & \operatorname{Pr}\left[\frac{1}{M}\left|\left\{i \in[M]: \exists \mathcal{L} \in \bigcup_{k \in[K]} \pi_{k}\left(\begin{array}{c}
i-1] \\
L
\end{array}\right), \tau_{\underline{u}, \underline{\mathbf{x}}_{i}, \underline{\mathbf{x}}_{\mathcal{L}}, \underline{s}}=P_{\mathbf{u}, \mathbf{x}, \mathbf{x}_{[L]}, \mathbf{s}}\right\}\right|>2^{-n \varepsilon / 2}\right] \\
= & \operatorname{Pr}\left[\frac{1}{M}\left|\left\{i \in[M]: \exists k \in[K], \exists \mathcal{L} \in \pi_{k}\left(\begin{array}{c}
{[i-1]} \\
L
\end{array}\right), \tau_{\underline{u}, \underline{\mathbf{x}}_{i}, \underline{\mathbf{x}}_{\mathcal{L}}, \underline{\underline{s}}}=P_{\mathbf{u}, \mathbf{x}, \mathbf{x}_{[L]}, \mathbf{s}}\right\}\right|>2^{-n \varepsilon / 2}\right] \\
= & \operatorname{Pr}\left[\frac{1}{M}\left|\bigcup_{k \in[K]}\left\{i \in[M]: \exists \mathcal{L} \in \pi_{k}\left(\begin{array}{c}
{[i-1]} \\
L
\end{array}\right), \tau_{\underline{u}, \underline{\mathbf{x}}_{i}, \underline{\mathbf{x}}_{\mathcal{L}}, \underline{\underline{s}}}=P_{\mathbf{u}, \mathbf{x}, \mathbf{x}_{[L]}, \mathbf{s}}\right\}\right|>2^{-n \varepsilon / 2}\right] \\
\leqslant & \operatorname{Pr}\left[\frac{1}{M} \sum_{k \in[K]}\left|\left\{i \in[M]: \exists \mathcal{L} \in \pi_{k}\left(\begin{array}{c}
{[i-1]} \\
L
\end{array}\right), \tau_{\underline{u}, \underline{\mathbf{x}}_{i}, \underline{\mathbf{x}}_{\mathcal{L}}, \underline{s}}=P_{\mathbf{u}, \mathbf{x}, \mathbf{x}_{[L]}, \mathbf{s}}\right\}\right|>2^{-n \varepsilon / 2}\right]
\end{aligned}
$$




$$
\begin{aligned}
& \leqslant \sum_{k \in[K]} \operatorname{Pr}\left[\frac{1}{M}\left|\left\{i \in[M]: \exists \mathcal{L} \in \pi_{k}\left(\begin{array}{c}
{[i-1]} \\
L
\end{array}\right), \tau_{\underline{u}, \underline{\mathbf{x}}_{i}, \underline{\mathbf{x}}_{\mathcal{L}}, \underline{s}}=P_{\mathbf{u}, \mathbf{x}, \mathbf{x}_{[L]}, \mathbf{s}}\right\}\right|>2^{-n \varepsilon / 2} / K\right] \\
& \leqslant 2^{n \varepsilon / 6} \operatorname{Pr}\left[\frac{1}{M}\left|\left\{i \in[M]: \exists \mathcal{L} \in\left(\begin{array}{c}
{[i-1]} \\
L
\end{array}\right), \tau_{\underline{u}, \underline{\mathbf{x}}_{i}, \underline{\mathbf{x}}_{\mathcal{L}}, \underline{s}}=P_{\mathbf{u}, \mathbf{x}, \mathbf{x}_{[L]}, \mathbf{s}}\right\}\right|>2^{\left.-\frac{2}{3} n \varepsilon\right]}\right. \\
& \leqslant(L+1) 2^{-2^{\frac{n \varepsilon}{4 L}}+n \varepsilon / 6} .
\end{aligned}
$$

The last inequality is by Equation (102).

e) Proof of Equation (14): Under the condition $I\left(\mathbf{x} ; \mathbf{x}_{k}, \mathbf{s} \mid \mathbf{u}\right)-\left[R-I\left(\mathbf{x}_{k} ; \mathbf{s} \mid \mathbf{u}\right)\right]^{+} \geqslant \varepsilon$, Equation (14) follows by setting $L=1$ and $\varepsilon_{1}=\left[R-I\left(\mathbf{x}_{k} ; \mathbf{s} \mid \mathbf{u}\right)\right]^{+}+\varepsilon / 4$. Specifically, by repeating the proof of Equation (16), we have the following bound similar to Equation (103).

$$
\operatorname{Pr}\left[\frac{1}{M}\left|\left\{i \in[M]: \exists j \in[M] \backslash\{i\}, \tau_{\underline{u}, \underline{\mathbf{x}}_{i}, \underline{\mathbf{x}}_{j}, \underline{s}}=P_{\mathbf{u}, \mathbf{x}, \mathbf{x}_{k}, \mathbf{s}}\right\}\right|>2^{-n \varepsilon / 2}\right] \leqslant 2 \cdot 2^{-\frac{1}{2} 2^{n \varepsilon / 4}+n \varepsilon / 6} .
$$

f) Finishing up the proof of Lemma 19: Finally, Lemma 19 follows by taking a union bound over $\underline{x} \in \mathcal{X}^{n}$ such that $\tau_{u, x}=P_{\mathbf{u}, \mathbf{x}}, \underline{s} \in \mathcal{S}^{n}$ and joint types $P_{\mathbf{u}, \mathbf{x}, \mathbf{x}_{[L]}, \mathbf{s}}$. There are in total exponentially many of them and the concentration bounds in Equation (91), Equation (94), Equation (95), Equation (103) are all doubly exponentially small. We thus have shown that with probability doubly exponentially close to 1 , a random code consisting of codewords $\underline{\mathbf{x}}_{1}, \cdots, \underline{\mathbf{x}}_{M} \in \mathcal{X}^{n}$ each of type $\tau_{\underline{u}, \underline{\mathbf{x}}_{i}}=P_{\mathbf{u}, \mathbf{x}}(i \in[M])$ simultaneously satisfies Equation (13), Equation (14), Equation (15), Equation (16) and Equation (17) for all $\underline{x}, \underline{s}, P_{\mathbf{x}, \mathbf{x}_{[L]}, \mathbf{s}}$.

\section{APPENDIX D}

\section{UNAMBIGUITY OF DECODING RULES (PROOF OF LEMMA 20)}

The proof is by contradiction. Suppose there does exist a joint distribution $P_{\mathbf{u}, \mathbf{x}_{[L+1]}, \mathbf{s}_{[L+1]}, \mathbf{y}}$ satisfying the conditions in Equation (19). Observe that for any $i \in[L+1]$,

$$
\begin{aligned}
& 2 \eta \geqslant D\left(P_{\mathbf{u}, \mathbf{x}_{i}, \mathbf{s}_{i}, \mathbf{y}} \| P_{\mathbf{u}} P_{\mathbf{x}_{i} \mid \mathbf{u}} P_{\mathbf{s}_{i}} W_{\mathbf{y} \mid \mathbf{x}, \mathbf{s}}\right)+I\left(\mathbf{x}_{i}, \mathbf{y} ; \mathbf{x}_{[L+1] \backslash\{i\}} \mid \mathbf{u}, \mathbf{s}_{i}\right)=D\left(P_{\mathbf{u}, \mathbf{x}_{i}, \mathbf{x}_{[L+1] \backslash\{i\}}, \mathbf{s}_{i}, \mathbf{y} \|} \| P_{\mathbf{u}} P_{\mathbf{x}_{i} \mid \mathbf{u}} P_{\mathbf{x}_{[L+1] \backslash\{i\}}, \mathbf{s}_{i} \mid \mathbf{u}} W_{\mathbf{y} \mid \mathbf{x}, \mathbf{s}}\right) \\
& \geqslant D\left(P_{\mathbf{u}, \mathbf{x}_{i}, \mathbf{x}_{[L+1] \backslash\{i\}}, \mathbf{y}} \| P_{\mathbf{u}} P_{\mathbf{x}_{i} \mid \mathbf{u}} V_{\mathbf{x}_{[L+1] \backslash\{i\}}, \mathbf{y} \mid \mathbf{u}, \mathbf{x}_{i}}\right) .
\end{aligned}
$$

where

$$
V_{\mathbf{x}_{[L+1] \backslash\{i\}}, \mathbf{y} \mid \mathbf{u}, \mathbf{x}_{i}}:=\left[P_{\mathbf{x}_{[L+1] \backslash\{i\}}, \mathbf{s}_{i} \mid \mathbf{u}} W_{\mathbf{y} \mid \mathbf{x}, \mathbf{s}}\right]_{\mathbf{x}_{[L+1] \backslash\{i\}}, \mathbf{y} \mid \mathbf{u}, \mathbf{x}_{i}} .
$$

By Pinsker's inequality (Lemma 5),

$$
2 \sqrt{\ln 2} \sqrt{\eta} \geqslant\left\|P_{\mathbf{u}, \mathbf{x}_{i}, \mathbf{x}_{[L+1] \backslash\{i\}}, \mathbf{y}}-P_{\mathbf{u}} P_{\mathbf{x}_{i} \mid \mathbf{u}} V_{\mathbf{x}_{[L+1] \backslash\{i\}}, \mathbf{y} \mid \mathbf{u}, \mathbf{x}_{i}}\right\|_{1} .
$$

The same bound as Equation (105) with $i$ replaced by $i^{\prime} \in[L+1] \backslash\{i\}$ still holds. Adding up both sides of these two bounds and applying triangle inequality on the RHS, we obtain

$$
\begin{aligned}
& 4 \sqrt{\ln 2} \sqrt{\eta} \geqslant\left\|P_{\mathbf{u}} P_{\mathbf{x}_{i} \mid \mathbf{u}} V_{\mathbf{x}_{[L+1] \backslash\{i}, \mathbf{y} \mid \mathbf{u}, \mathbf{x}_{i}}-P_{\mathbf{u}} P_{\mathbf{x}_{i^{\prime}} \mid \mathbf{u}} V_{\left.\mathbf{x}_{[L+1] \backslash\left\{i^{\prime}\right.}\right\}}, \mathbf{y} \mid \mathbf{u}, \mathbf{x}_{i^{\prime}}\right\|_{1} \\
& \geqslant p_{u}^{*}|\mathcal{U}|\left\|P_{\mathbf{x}_{i} \mid \mathbf{u}=u^{*}} V_{\mathbf{x}_{[L+1] \backslash\{i\}}, \mathbf{y} \mid \mathbf{u}=u^{*}, \mathbf{x}_{i}}-P_{\mathbf{x}_{i^{\prime}} \mid \mathbf{u}=u^{*}} V_{\left.\mathbf{x}_{[L+1] \backslash\left\{i^{\prime}\right.}\right\}}, \mathbf{y} \mid \mathbf{u}=u^{*}, \mathbf{x}_{i^{\prime}}\right\|_{1} \\
& =p_{u}^{*}|\mathcal{U}| \sum_{x_{[L+1]} \in \mathcal{X}^{L+1}} \sum_{y \in \mathcal{Y}} \mid P_{\mathbf{x} \mid \mathbf{u}}\left(x_{i} \mid u^{*}\right) V_{\mathbf{x}_{[L+1] \backslash\{i\}}, \mathbf{y} \mid \mathbf{u}, \mathbf{x}_{i}}\left(x_{[L+1] \backslash\{i\}}, y \mid u^{*}, x_{i}\right) \\
& -P_{\mathbf{x} \mid \mathbf{u}}\left(x_{i^{\prime}} \mid u^{*}\right) V_{\mathbf{x}_{[L+1] \backslash\left\{i^{\prime}\right\}}, \mathbf{y} \mid \mathbf{u}, \mathbf{x}_{i^{\prime}}}\left(x_{[L+1] \backslash\left\{i^{\prime}\right\}}, y \mid u^{*}, x_{i^{\prime}}\right) \mid \\
& =p_{u}^{*}|\mathcal{U}| \frac{1}{(L+1) !} \sum_{\pi \in S_{L+1}} \sum_{x_{[L+1]} \in \mathcal{X}^{L+1}} \sum_{y \in \mathcal{Y}} \mid P_{\mathbf{x} \mid \mathbf{u}}\left(x_{i} \mid u^{*}\right) V_{\mathbf{x}_{[L+1] \backslash\{\pi(i)\}}, \mathbf{y} \mid \mathbf{u}, \mathbf{x}_{\pi(i)}}\left(x_{\pi([L+1]) \backslash\{i\}}, y \mid u^{*}, x_{i}\right) \\
& -P_{\mathbf{x} \mid \mathbf{u}}\left(x_{i^{\prime}} \mid u^{*}\right) V_{\mathbf{x}_{[L+1] \backslash\left\{\pi\left(i^{\prime}\right)\right\}}, \mathbf{y} \mid \mathbf{u}, \mathbf{x}_{\pi\left(i^{\prime}\right)}}\left(x_{\pi([L+1]) \backslash\left\{i^{\prime}\right\}}, y \mid u^{*}, x_{i^{\prime}}\right) \mid \\
& =p_{u}^{*}|\mathcal{U}| \frac{1}{(L+1) !} \sum_{\pi \in S_{L+1}} \sum_{x_{[L+1]} \in \mathcal{X}^{L+1}} \sum_{y \in \mathcal{Y}} \mid \sum_{s \in \mathcal{S}}\left(P_{\mathbf{x} \mid \mathbf{u}}\left(x_{i} \mid u^{*}\right) P_{\mathbf{x}_{[L+1] \backslash\{\pi(i)\}}, \mathbf{s}_{\pi(i)} \mid \mathbf{u}}\left(x_{\pi([L+1]) \backslash\{i\}}, s \mid u^{*}\right) W_{\mathbf{y} \mid \mathbf{x}, \mathbf{s}}\left(y \mid x_{i}, s\right)\right.
\end{aligned}
$$




$$
\begin{aligned}
& \left.-P_{\mathbf{x} \mid \mathbf{u}}\left(x_{i^{\prime}} \mid u^{*}\right) P_{\mathbf{x}_{[L+1] \backslash\left\{\pi\left(i^{\prime}\right)\right\}}, \mathbf{s}_{\pi\left(i^{\prime}\right)} \mid \mathbf{u}}\left(x_{\pi([L+1]) \backslash\left\{i^{\prime}\right\}}, s \mid u^{*}\right) W_{\mathbf{y} \mid \mathbf{x}, \mathbf{s}}\left(y \mid x_{i^{\prime}}, s\right)\right) \mid \\
\geqslant & p_{u}^{*}|\mathcal{U}| \sum_{x_{[L+1]} \in \mathcal{X}^{L+1}} \sum_{y \in \mathcal{Y}} \mid \sum_{s \in \mathcal{S}}\left(P_{\mathbf{x} \mid \mathbf{u}}\left(x_{i} \mid u^{*}\right) Q_{\mathbf{x}_{[L]}, \mathbf{s} \mid \mathbf{u}}\left(x_{[L+1] \backslash\{i\}}, s \mid u^{*}\right) W_{\mathbf{y} \mid \mathbf{x}, \mathbf{s}}\left(y \mid x_{i}, s\right)\right. \\
& \left.-P_{\mathbf{x} \mid \mathbf{u}}\left(x_{i^{\prime}} \mid u^{*}\right) Q_{\mathbf{x}_{[L]}, \mathbf{s} \mid \mathbf{u}}\left(x_{[L+1] \backslash\left\{i^{\prime}\right\}}, s \mid u^{*}\right) W_{\mathbf{y} \mid \mathbf{x}, \mathbf{s}}\left(y \mid x_{i^{\prime}}, s\right)\right) \mid
\end{aligned}
$$

where in Equation (106) we use the following notation

$$
p_{u}^{*}:=\min _{u \in \mathcal{U}} P_{\mathbf{u}}(u), u^{*}:=\underset{u \in \mathcal{U}}{\operatorname{argmin}}\left\|P_{\mathbf{x} \mid \mathbf{u}=u} V_{\mathbf{x}_{[L+1] \backslash\{i\}}, \mathbf{y} \mid \mathbf{u}=u, \mathbf{x}_{i}}-P_{\mathbf{x} \mid \mathbf{u}=u} V_{\mathbf{x}_{[L+1] \backslash\left\{i^{\prime}\right\}}, \mathbf{y} \mid \mathbf{u}=u, \mathbf{x}_{i^{\prime}}}\right\|_{1} .
$$

In Equation (109) we define

$$
Q_{\mathbf{x}_{[L]}, \mathbf{s} \mid \mathbf{u}}\left(x_{[L]}, s \mid u\right):=\frac{1}{(L+1) !} \sum_{\pi^{\prime} \in S_{L}} \sum_{j \in[L+1]} P_{\mathbf{x}_{[L+1] \backslash\{j\}}, \mathbf{s}_{j} \mid \mathbf{u}}\left(x_{\pi^{\prime}([L])}, s \mid u\right),
$$

which is due to the following identity:

$$
\begin{aligned}
Q_{\mathbf{x}_{[L]}, \mathbf{s} \mid \mathbf{u}}\left(x_{[L+1] \backslash\{i\}}, s \mid u^{*}\right) & =\frac{1}{(L+1) !} \sum_{\pi \in S_{L+1}} P_{\mathbf{x}_{[L+1] \backslash\{\pi(i)\}}, \mathbf{s}_{\pi(i)} \mid \mathbf{u}}\left(x_{\pi([L+1]) \backslash\{i\}}, s \mid u^{*}\right) \\
& =\frac{1}{(L+1) !} \sum_{\pi^{\prime} \in S_{L}} \sum_{j \in[L+1]} P_{\mathbf{x}_{[L+1] \backslash\{j\}}, \mathbf{s}_{j} \mid \mathbf{u}}\left(x_{\pi^{\prime}([L+1] \backslash\{i\})}, s \mid u^{*}\right) .
\end{aligned}
$$

Equation (107) follows since $P_{\mathbf{u}, \mathbf{x}_{i}}=P_{\mathbf{u}, \mathbf{x}}$ for all $i \in[L+1]$. Equation (108) follows since Equation (107) is invariant under any permutation $\pi \in S_{L+1}$. Equation (109) follows from triangle inequality.

We observe that $Q_{\mathbf{x}_{[L]}, \mathbf{s} \mid \mathbf{u}}$ is symmetric in $\mathbf{x}_{[L]}$. Indeed, for any $\sigma \in S_{L}$,

$$
\begin{aligned}
Q_{\mathbf{x}_{[L]}, \mathbf{s} \mid \mathbf{u}}\left(x_{\sigma([L])}, s \mid u\right) & =\frac{1}{(L+1) !} \sum_{\pi^{\prime} \in S_{L}} \sum_{j \in[L+1]} P_{\mathbf{x}_{[L+1] \backslash\{j\}}, \mathbf{s}_{j} \mid \mathbf{u}}\left(x_{\sigma\left(\pi^{\prime}([L])\right)}, s \mid u\right) \\
& =\frac{1}{(L+1) !} \sum_{\pi^{\prime} \in S_{L}} \sum_{j \in[L+1]} P_{\mathbf{x}_{[L+1] \backslash\{j\}}, \mathbf{s}_{j} \mid \mathbf{u}}\left(x_{\left(\sigma \pi^{\prime}\right)([L])}, s \mid u\right) \\
& =Q_{\mathbf{x}_{[L]}, \mathbf{s} \mid \mathbf{u}}\left(x_{[L]}, s \mid u\right) .
\end{aligned}
$$

We claim that Equation (109) must be strictly positive. Otherwise,

$$
\begin{aligned}
& \sum_{s \in \mathcal{S}} P_{\mathbf{x} \mid \mathbf{u}}\left(x_{i} \mid u^{*}\right) Q_{\mathbf{x}_{[L]}, \mathbf{s} \mid \mathbf{u}}\left(x_{[L+1] \backslash\{i\}}, s \mid u^{*}\right) W_{\mathbf{y} \mid \mathbf{x}, \mathbf{s}}\left(y \mid x_{i}, s\right) \\
= & \sum_{s \in \mathcal{S}} P_{\mathbf{x} \mid \mathbf{u}}\left(x_{i^{\prime}} \mid u^{*}\right) Q_{\mathbf{x}_{[L]}, \mathbf{s} \mid \mathbf{u}}\left(x_{[L+1] \backslash\left\{i^{\prime}\right\}}, s \mid u^{*}\right) W_{\mathbf{y} \mid \mathbf{x}, \mathbf{s}}\left(y \mid x_{i^{\prime}}, s\right) \\
\Longrightarrow \quad & \sum_{s \in \mathcal{S}} P_{\mathbf{x} \mid \mathbf{u}}\left(x_{i} \mid u^{*}\right) Q_{\mathbf{x}_{[L]}, \mathbf{s} \mid \mathbf{u}}\left(x_{[L+1] \backslash\{i\}}, s \mid u^{*}\right)=\sum_{s \in \mathcal{S}} P_{\mathbf{x} \mid \mathbf{u}}\left(x_{i^{\prime}} \mid u^{*}\right) Q_{\mathbf{x}_{[L]}, \mathbf{s} \mid \mathbf{u}}\left(x_{[L+1] \backslash\left\{i^{\prime}\right\}}, s \mid u^{*}\right) \\
\Longrightarrow \quad & P_{\mathbf{x} \mid \mathbf{u}}\left(x_{i} \mid u^{*}\right) Q_{\mathbf{x}_{[L]} \mid \mathbf{u}}\left(x_{[L+1] \backslash\{i\}} \mid u^{*}\right)=P_{\mathbf{x} \mid \mathbf{u}}\left(x_{i^{\prime}} \mid u^{*}\right) Q_{\mathbf{x}_{[L]} \mid \mathbf{u}}\left(x_{\left.[L+1] \backslash i^{\prime}\right\}} \mid u^{*}\right)
\end{aligned}
$$

In fact, $Q$ satisfying Equation (111) must be a product distribution $Q_{\mathbf{x}_{[L]} \mid \mathbf{u}=u^{*}}=P_{\mathbf{x} \mid \mathbf{u}=u^{*}}^{\otimes}$ and is obviously symmetric. This is follows from Lemma 34 which is stated at the end of this section. A proof can be found in [Hug97]. Substituting $Q$ back to Equation (110), we get

$$
\begin{aligned}
& \sum_{s \in \mathcal{S}} P_{\mathbf{x} \mid \mathbf{u}}\left(x_{i} \mid u^{*}\right) Q_{\mathbf{x}_{[L]} \mid \mathbf{u}}\left(x_{[L+1] \backslash\{i\}} \mid u^{*}\right) Q_{\mathbf{s} \mid \mathbf{x}_{[L]}, \mathbf{u}}\left(s \mid x_{[L+1] \backslash\{i\}}, u^{*}\right) W_{\mathbf{y} \mid \mathbf{x}, \mathbf{s}}\left(y \mid x_{i}, s\right) \\
= & \sum_{s \in \mathcal{S}} P_{\mathbf{x} \mid \mathbf{u}}\left(x_{i^{\prime}} \mid u^{*}\right) Q_{\mathbf{x}_{[L]} \mid \mathbf{u}}\left(x_{[L+1] \backslash\left\{i^{\prime}\right\}} \mid u^{*}\right) Q_{\mathbf{s} \mid \mathbf{x}_{[L]}, \mathbf{u}}\left(s \mid x_{[L+1] \backslash\left\{i^{\prime}\right\}}, u^{*}\right) W_{\mathbf{y} \mid \mathbf{x}, \mathbf{s}}\left(y \mid x_{i^{\prime}}, s\right) \\
\Longrightarrow \quad & \sum_{s \in \mathcal{S}} P_{\mathbf{x}_{i} \mid \mathbf{u}}\left(x_{i} \mid u^{*}\right) P_{\mathbf{x} \mid \mathbf{u}}^{\otimes L}\left(x_{[L+1] \backslash\{i\}} \mid u^{*}\right) Q_{\mathbf{s} \mid \mathbf{x}_{[L]}, \mathbf{u}}\left(s \mid x_{[L+1] \backslash\{i\}}, u^{*}\right) W_{\mathbf{y} \mid \mathbf{x}, \mathbf{s}}\left(y \mid x_{i}, s\right) \\
= & \sum_{s \in \mathcal{S}} P_{\mathbf{x}_{i^{\prime}} \mid \mathbf{u}}\left(x_{i^{\prime}} \mid u^{*}\right) P_{\mathbf{x} \mid \mathbf{u}}^{\otimes L}\left(x_{[L+1] \backslash\left\{i^{\prime}\right\}} \mid u^{*}\right) Q_{\mathbf{s} \mid \mathbf{x}_{[L]}, \mathbf{u}}\left(s \mid x_{[L+1] \backslash\left\{i^{\prime}\right\}}, u^{*}\right) W_{\mathbf{y} \mid \mathbf{x}, \mathbf{s}}\left(y \mid x_{i^{\prime}}, s\right)
\end{aligned}
$$




$$
\begin{aligned}
\Longrightarrow \quad & \sum_{s \in \mathcal{S}} P_{\mathbf{x} \mid \mathbf{u}}^{\otimes(L+1)}\left(x_{[L+1]} \mid u^{*}\right) Q_{\mathbf{s} \mid \mathbf{x}_{[L]}, \mathbf{u}}\left(s \mid x_{[L+1] \backslash\{i\}}, u^{*}\right) W_{\mathbf{y} \mid \mathbf{x}, \mathbf{s}}\left(y \mid x_{i}, s\right) \\
= & \sum_{s \in \mathcal{S}} P_{\mathbf{x} \mid \mathbf{u}}^{\otimes(L+1)}\left(x_{[L+1]} \mid u^{*}\right) Q_{\mathbf{s} \mid \mathbf{x}_{[L]}, \mathbf{u}}\left(s \mid x_{[L+1] \backslash\left\{i^{\prime}\right\}}, u^{*}\right) W_{\mathbf{y} \mid \mathbf{x}, \mathbf{s}}\left(y \mid x_{i^{\prime}}, s\right) \\
\Longrightarrow \quad & \sum_{s \in \mathcal{S}} Q_{\mathbf{s} \mid \mathbf{x}_{[L]}, \mathbf{u}}\left(s \mid x_{[L+1] \backslash\{i\}}, u^{*}\right) W_{\mathbf{y} \mid \mathbf{x}, \mathbf{s}}\left(y \mid x_{i}, s\right)=\sum_{s \in \mathcal{S}} Q_{\mathbf{s} \mid \mathbf{x}_{[L]}, \mathbf{u}}\left(s \mid x_{[L+1] \backslash\left\{i^{\prime}\right\}}, u^{*}\right) W_{\mathbf{y} \mid \mathbf{x}, \mathbf{s}}\left(y \mid x_{i^{\prime}}, s\right) .
\end{aligned}
$$

Note that $Q_{\mathbf{s} \mid \mathbf{x}_{[L]}, \mathbf{u}}=Q_{\mathbf{s}, \mathbf{x}_{[L]}, \mathbf{u}} / Q_{\mathbf{x}_{[L]}, \mathbf{u}}$, and both $Q_{\mathbf{s}, \mathbf{x}_{[L]}, \mathbf{u}}, Q_{\mathbf{x}_{[L]}, \mathbf{u}}$ are symmetric in $\mathbf{x}_{[L]}$. Therefore $Q_{\mathbf{s} \mid \mathbf{x}_{[L]}, \mathbf{u}}$ is also symmetric in $\mathbf{x}_{[L]}$. Combining this observation with Equation (112), we know that $Q_{\mathbf{s} \mid \mathbf{x}_{[L]}, \mathbf{u}} \in \mathcal{U}_{\text {obli,L-symm. }}$.

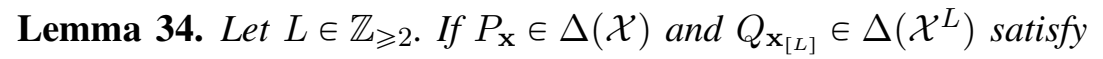

$$
P_{\mathbf{x}}\left(x_{i}\right) Q_{\mathbf{x}_{[L]}}\left(x_{[L+1] \backslash\{i\}}\right)=P_{\mathbf{x}}\left(x_{i^{\prime}} \mid u\right) Q_{\mathbf{x}_{[L]}}\left(x_{[L+1] \backslash\left\{i^{\prime}\right\}}\right)
$$

for all $i \neq i^{\prime} \in[L]$ and $x_{[L]} \in \mathcal{X}^{L}$, then $Q_{\mathbf{x}_{[L]}}=P_{\mathbf{x}}^{\otimes L}$.

\section{APPENDIX E}

\section{STRONG CONVERSE FOR FADING DMCS WITH APPROXIMATE CONSTANT-COMPOSITION CODES AND LIST-DECODING (PROOF OF THEOREM 23)}

Let $\mathcal{C}$ be a code of rate $R=C\left(W_{\mathbf{y} \mid \mathbf{x}, \mathbf{u}}\right)+\delta$ and let $L \in \mathbb{Z}_{\geqslant 1}$ be the list-size. Let $P_{\mathbf{u}}=\tau_{\underline{u}}$. Suppose that for some $P_{\mathbf{x} \mid \mathbf{u}}$, for all $\underline{x} \in \mathcal{C}, d\left(\tau_{\underline{u}, \underline{x}}, P_{\mathbf{u}} P_{\mathbf{x} \mid \mathbf{u}}\right) \leqslant \lambda$ where $0<\lambda \ll \delta$ is a constant. Let $\psi: \mathcal{Y}^{n} \rightarrow\left(\begin{array}{c}{[M]} \\ \leqslant L\end{array}\right)$ be the optimal list-decoder of $\mathcal{C}$ used over $W_{\mathbf{y} \mid \mathbf{x}, \mathbf{u}}$.

Let $\varepsilon>0$ be a sufficiently small constant to be determined later. Define the $\varepsilon$-typical set as

$$
\mathcal{A}_{\underline{\mathbf{y}} \mid \underline{u}}^{\varepsilon}\left(P_{\mathbf{y} \mid \mathbf{u}}\right):=\left\{\underline{y} \in \mathcal{Y}^{n}: \forall u \in \mathcal{U}, \forall y \in \mathcal{Y}, \frac{\tau_{\underline{y} \mid \underline{u}}(y \mid u)}{P_{\mathbf{y} \mid \mathbf{u}}(y \mid u)} \in[1-\varepsilon, 1+\varepsilon]\right\},
$$

where $P_{\mathbf{y} \mid \mathbf{u}}=\left[P_{\mathbf{x} \mid \mathbf{u}} W_{\mathbf{y} \mid \mathbf{x}, \mathbf{u}}\right]_{\mathbf{y} \mid \mathbf{u}}$. Note that by the asymptotic equipartition property (Lemma 9)

$$
\left|\mathcal{A}_{\underline{\mathbf{y}} \mid \underline{u}}^{\varepsilon}\left(P_{\mathbf{y} \mid \mathbf{u}}\right)\right| \leqslant 2^{n\left(H(\mathbf{y} \mid \mathbf{u})+f_{2}(\lambda, \varepsilon)\right)},
$$

for some $f_{2}(\lambda, \varepsilon)>0$.

We now lower bound the average error probability.

$$
\begin{aligned}
& 1-P_{\mathrm{e}, \mathrm{avg}}(\mathcal{C})=\frac{1}{M} \sum_{i \in[M]} \operatorname{Pr}[\psi(\underline{\mathbf{y}}) \ni i \mid \mathbf{m}=i, \underline{\mathbf{u}}=\underline{u}] \\
& =\frac{1}{M} \sum_{i \in[M]} \sum_{y \in \mathcal{Y}^{n}} W_{\mathbf{y} \mid \mathbf{x}, \mathbf{u}}^{\otimes n}\left(\underline{y} \mid \underline{x}_{i}, \underline{u}\right) \mathbb{1}\{\psi(\underline{y}) \ni i\} \\
& \leqslant \frac{1}{M} \sum_{i \in[M]} \sum_{\underline{y} \in \mathcal{A}_{\underline{\mathbf{y}} \mid \underline{u}}^{\varepsilon}\left(P_{\mathbf{y} \mid \mathbf{u}}\right)} W_{\mathbf{y} \mid \mathbf{x}, \mathbf{u}}^{\otimes n}\left(\underline{y} \underline{x}_{i}, \underline{u}\right) \mathbb{1}\{\psi(\underline{y}) \ni i\}+\frac{1}{M} \sum_{i \in[M]} \sum_{y \neq \mathcal{A}_{\underline{\mathbf{y}} \mid \underline{u}}^{\varepsilon}\left(P_{\mathbf{y} \mid \mathbf{u}}\right)} W_{\mathbf{y} \mid \mathbf{x}, \mathbf{u}}^{\otimes n}\left(\underline{y} \mid \underline{x}_{i}, \underline{u}\right) .
\end{aligned}
$$

We claim that $\underline{x}_{i} \in \mathcal{C}$ satisfies $d\left(\tau_{\underline{x}_{i} \mid u}, P_{\mathbf{x} \mid \mathbf{u}=u}\right) \leqslant \lambda^{\prime}$ for all $u \in \mathcal{U}$, where $\lambda^{\prime}=\frac{\lambda}{|\mathcal{U}| p_{u}^{*}}$. This is guaranteed by approximate constant-composition of $\mathcal{C}$. Indeed for any $\underline{x} \in \mathcal{C}$,

$$
\begin{aligned}
\lambda \geqslant & \geqslant\left(\tau_{\underline{u}, \underline{x}}, P_{\mathbf{u}} P_{\mathbf{x} \mid \mathbf{u}}\right) \\
& =\sum_{(u, x) \in \mathcal{U} \times \mathcal{X}}\left|\tau_{\underline{u}, \underline{x}}(u, x)-P_{\mathbf{u}} P_{\mathbf{x} \mid \mathbf{u}}(u, x)\right| \\
& =\sum_{(u, x) \in \mathcal{U} \times \mathcal{X}}\left|\tau_{\underline{u}}(u) \tau_{\underline{x}}\right| \underline{u}(x \mid u)-P_{\mathbf{u}}(u) P_{\mathbf{x} \mid \mathbf{u}}(x \mid u) \mid \\
& =\sum_{(u, x) \in \mathcal{U} \times \mathcal{X}} P_{\mathbf{u}}(u)\left|\tau_{\underline{x}}\right| \underline{u}(x \mid u)-P_{\mathbf{x} \mid \mathbf{u}}(x \mid u) \mid \\
& =\sum_{u \in \mathcal{U}} P_{\mathbf{u}}(u) \sum_{x \in \mathcal{X}}\left|\tau_{\underline{x}}\right| u(x)-P_{\mathbf{x} \mid \mathbf{u}=u}(x) \mid
\end{aligned}
$$




$$
\begin{aligned}
& =\sum_{u \in \mathcal{U}} P_{\mathbf{u}}(u) d\left(\tau_{\underline{x} \mid u}, P_{\mathbf{x} \mid \mathbf{u}=u}\right) \\
& \geqslant|\mathcal{U}| p_{u}^{*} d\left(\tau_{\underline{x} \mid u}, P_{\mathbf{x} \mid \mathbf{u}=u}\right),
\end{aligned}
$$

where Equation (114) holds for any $u \in \mathcal{U}$ and $p_{u}^{*}$ is defined as $p_{u}^{*}:=\min _{u \in \mathcal{U}} P_{\mathbf{u}}(u)>0$ (since $P_{\mathbf{u}}=\tau_{\underline{u}}$ has no zero atoms).

Hence for any $\underline{y} \in \mathcal{A}_{\underline{\mathbf{y}} \mid \underline{u}}^{\varepsilon}\left(P_{\mathbf{y} \mid \mathbf{u}}\right)$,

$$
W_{\mathbf{y} \mid \mathbf{x}, \mathbf{u}}^{\otimes n}\left(\underline{y} \mid \underline{x}_{i}, \underline{u}\right) \leqslant 2^{-n\left(H(\mathbf{y} \mid \mathbf{x}, \mathbf{u})-f_{1}(\lambda, \varepsilon)\right)},
$$

for some $f_{1}(\lambda, \varepsilon)>0$. Thus the first term in Equation (113) is at most

$$
\begin{aligned}
\frac{1}{M} 2^{-n\left(H(\mathbf{y} \mid \mathbf{x}, \mathbf{u})-f_{1}(\lambda, \varepsilon)\right)} \sum_{\underline{y} \in \mathcal{A}_{\underline{\underline{\varepsilon}} \mid \underline{u}}^{\varepsilon}\left(P_{\mathbf{y} \mid \mathbf{u}}\right)} \sum_{i \in[M]} \mathbb{1}\{\psi(\underline{y}) \ni i\} & \leqslant \frac{1}{M} 2^{-n\left(H(\mathbf{y} \mid \mathbf{x}, \mathbf{u})-f_{1}(\lambda, \varepsilon)\right)}\left|\mathcal{A}_{\underline{\mathbf{y}} \mid \underline{u}}^{\varepsilon}\left(P_{\mathbf{y} \mid \mathbf{u}}\right)\right| L \\
& \leqslant L^{-1} 2^{-n R} 2^{-n\left(H(\mathbf{y} \mid \mathbf{x}, \mathbf{u})-f_{1}(\lambda, \varepsilon)\right)} 2^{n\left(H(\mathbf{y} \mid \mathbf{u})+f_{2}(\lambda, \varepsilon)\right)} L \\
& \leqslant 2^{n\left(-C+I(\mathbf{x} ; \mathbf{y} \mid \mathbf{u})-\delta+f_{1}(\lambda, \varepsilon)+f_{2}(\lambda, \varepsilon)\right)} \\
& \leqslant 2^{-n\left(\delta-f_{1}(\lambda, \varepsilon)-f_{2}(\lambda, \varepsilon)\right)} .
\end{aligned}
$$

Let $\varepsilon$ be sufficiently small so that $\delta>f_{1}(\lambda, \varepsilon)+f_{2}(\lambda, \varepsilon)$. Then the bound in Equation (115) is exponentially decaying.

As for the second term in Equation (113), by the large deviation principle, we have that for any $\underline{x}_{i}$ satisfying $d\left(\tau_{\underline{x}_{i} \mid u}, P_{\mathbf{x} \mid \mathbf{u}=u}\right) \leqslant \lambda^{\prime}=\frac{\lambda}{|\mathcal{U}| p_{u}^{*}}$ for all $u \in \mathcal{U}$ (which is guaranteed by approximate constant-composition of $\mathcal{C}$ ),

$$
\operatorname{Pr}\left[\underline{\mathbf{y}} \notin \mathcal{A}_{\underline{\mathbf{y}} \mid \underline{u}}^{\varepsilon}\left(P_{\mathbf{y} \mid \mathbf{u}}\right) \mid \mathbf{m}=i, \underline{\mathbf{u}}=\underline{u}\right] \leqslant 2^{-n f_{3}(\lambda, \varepsilon)},
$$

for some $f_{3}(\lambda, \varepsilon)>0$.

Putting Equation (115) and Equation (116) back to Equation (113), we obtain

$$
P_{\mathrm{e}, \mathrm{avg}}(\mathcal{C}) \geqslant 1-2^{-n\left(\delta-f_{1}(\lambda, \varepsilon)-f_{2}(\lambda, \varepsilon)\right)}-2^{-n f_{3}(\lambda, \varepsilon)} \stackrel{n \rightarrow \infty}{\longrightarrow} 1,
$$

which finishes the proof of Theorem 23. 